\title{
Size dependent magnetic properties of nickel nanoparticles embedded in silica matrix
}

\author{
Vivek Singh \\ West Virginia University
}

Follow this and additional works at: https://researchrepository.wvu.edu/etd

\section{Recommended Citation}

Singh, Vivek, "Size dependent magnetic properties of nickel nanoparticles embedded in silica matrix" (2009). Graduate Theses, Dissertations, and Problem Reports. 2868.

https://researchrepository.wvu.edu/etd/2868

This Dissertation is protected by copyright and/or related rights. It has been brought to you by the The Research Repository @ WVU with permission from the rights-holder(s). You are free to use this Dissertation in any way that is permitted by the copyright and related rights legislation that applies to your use. For other uses you must obtain permission from the rights-holder(s) directly, unless additional rights are indicated by a Creative Commons license in the record and/ or on the work itself. This Dissertation has been accepted for inclusion in WVU Graduate Theses, Dissertations, and Problem Reports collection by an authorized administrator of The Research Repository @ WVU.

For more information, please contact researchrepository@mail.wvu.edu. 


\title{
SIZE DEPENDENT MAGNETIC PROPERTIES OF NICKEL NANOPARTICLES EMBEDDED IN SILICA MATRIX
}

\author{
Vivek Singh \\ DISSERTATION \\ Submitted to the Eberly College of Arts and Sciences \\ at \\ West Virginia University \\ in partial fulfillment of the requirements for the degree of \\ Doctor of Philosophy \\ in \\ Physics
Nancy Giles, Ph.D.
Larry Halliburton, Ph.D.
David Lederman, Ph.D.
Lloyd Carroll, Ph.D.
Department of Physics
Morgantown, West Virginia \\ Mohindar S. Seehra, Ph.D., Chair
}

2009

Keywords:

Nanoparticles, Superparamagnetism, Blocking Temperature, AC Susceptibility, Magnetic Relaxation, Coercivity 


\title{
ABSTRACT \\ SIZE DEPENDENT MAGNETIC PROPERTIES OF NICKEL NANOPARTICLES EMBEDDED IN SILICA MATRIX
}

\author{
Vivek Singh
}

In this dissertation, synthesis, structural and magnetic properties of nickel (Ni) nanoparticles (NPs) embedded in amorphous silica matrix are described in detail. These $\mathrm{Ni}$ NPs were prepared using the sol-gel technique. The percent composition of $x-\mathrm{Ni} / \mathrm{SiO}_{2}$ was varied from 1,5 and $15 \%$. Further, the samples were annealed in a furnace at temperatures between $400^{\circ}$ to $800^{\circ} \mathrm{C}$ for the duration of 2 hours in a continuous flow of ultra high pure (UHP) nitrogen in order to obtain different particle sizes ranging from 3.8 to $23 \mathrm{~nm}$ for the $15 \% \mathrm{Ni} / \mathrm{SiO}_{2}$ composition.

Structural characterization of the Ni NPs was done using transmission emission microscopy (TEM) and x-ray diffraction (XRD). Average particle sizes were obtained from the TEM micrographs by fitting them to log-normal distribution giving 3.8, 11.7, 15,21 and $23 \mathrm{~nm}$. The particle sizes were compared to those calculated from XRD patterns using the Debye-Scherrer equation.

Magnetic properties of these Ni NPs was studied using the superconducting quantum interference device (SQUID). The variations of the blocking temperature $\left(T_{B}\right)$ with measuring frequency $\left(\mathrm{f}_{\mathrm{m}}\right)$ and applied field $(\mathrm{H})$ are reported for Ni NPs with the nominal composition $\mathrm{Ni} / \mathrm{SiO}_{2}(15 / 85)$. Measurements from the variation in magnetization (M) vs. temperature (T) (2 to $350 \mathrm{~K}$ ) in $\mathrm{H}$ enabled us to determine the $\mathrm{T}_{\mathrm{B}}$ from the peaks in the zero field cooled curves. Measurements from $M$ vs. $H$ data above $T_{B}$ was fit to the modified Langevin function to obtain the magnetic moment per particle $\left(\mu_{\mathrm{P}}\right)$. The large value of the moment of the order of $\left(10^{3} \mu_{\mathrm{B}}\right)$ characterized these particles as superparamagnetic above $\mathrm{T}_{\mathrm{B}}$. Hysteresis measurements on cooling the sample in $\mathrm{H}$ were also done as a check for the presence of anti-ferromagnetic/ferromagnetic layer leading to exchange bias. Temperature dependence of AC susceptibility measurements were done for frequencies varying from 0.1 to $997 \mathrm{~Hz}$. The blocking temperatures $\mathrm{T}_{\mathrm{B}}$, as determined by peaks in $\chi^{\prime \prime}$ vs. $\mathrm{T}$ data, were fit to the Vogel-Fulcher law to determine the energy barrier and strength of the interparticle interaction.

The temperature dependence $(5$ to $300 \mathrm{~K}$ ) of the electron magnetic resonance (EMR) lines observed at $9.28 \mathrm{GHz}$ in $15 \% \mathrm{Ni} / \mathrm{SiO}_{2}$ nanocomposites with different particle sizes are also reported. In EMR, three resonance lines are observed: (i) Line 1 with linewidth $\Delta \mathrm{H} \simeq 50$ Oe and $\mathrm{g} \simeq 2$, and Curie-like variation of the line-intensity, with $\Delta \mathrm{H}$ and $\mathrm{g}$ being temperature and size-independent; (ii) Line 2 with $\Delta \mathrm{H} \simeq 850$ Oe and $\mathrm{g} \simeq 2.3$ for $\mathrm{D}=3.8 \mathrm{~nm}$ at $294 \mathrm{~K}$ with both $\Delta \mathrm{H}$ and $\mathrm{g}$ increasing with decreasing $\mathrm{T}$ and $\Delta \mathrm{H}$ size-dependent; and (iii) weak line 3 with $\mathrm{g} 4$ at $300 \mathrm{~K}$, with $\mathrm{g}$ also increasing with decreasing $\mathrm{T}$. We argue that the line 1 is due to dangling bonds in $\mathrm{SiO}_{2}$ as a similar line with $\Delta \mathrm{H} \simeq 9$ Oe is also observed in $\mathrm{SiO}_{2}$ without $\mathrm{Ni}$ doping. Lines 2 and 3 are attributed to majority Ni NPs and large Ni clusters respectively whose anisotropy is both size and temperature dependent, leading to the observed $\Delta \mathrm{H}$ and $\mathrm{g}$ values of the lines. 


\section{DEDICATION}

To my Parents. 


\section{ACKNOWLEDGEMENTS}

I take this moment to express my thank you to people who have always lend out their helping hand at all times. Dr. Mohindar S. Seehra, you have been a wonderful mentor to me in my research and academics. Your moral support and continuous guidance enabled me to complete my work successfully. I will always remember these years that you spent in teaching me experimental physics. I thank Dr. John Bonevich for carrying out the TEM studies reported in this work. I would like to thank my committee members Dr. Nancy Giles, Dr. Larry Halliburton, Dr. David Lederman and Dr. Lloyd Carroll for their time and effort. I am grateful to the professors in the Department of Physics at West Virginia University who have taught me physics courses. I also want to thank the departmental office staff and machine shop staff for their assistance. To all my friends, Mattias, Adi, Jorge, Erie, Akbar and James, thanks for being there. I am as ever especially thankful to my parents for their love and patience. Finally I thank the U. S. Department of Energy for supporting this project. 


\section{TABLE OF CONTENTS}

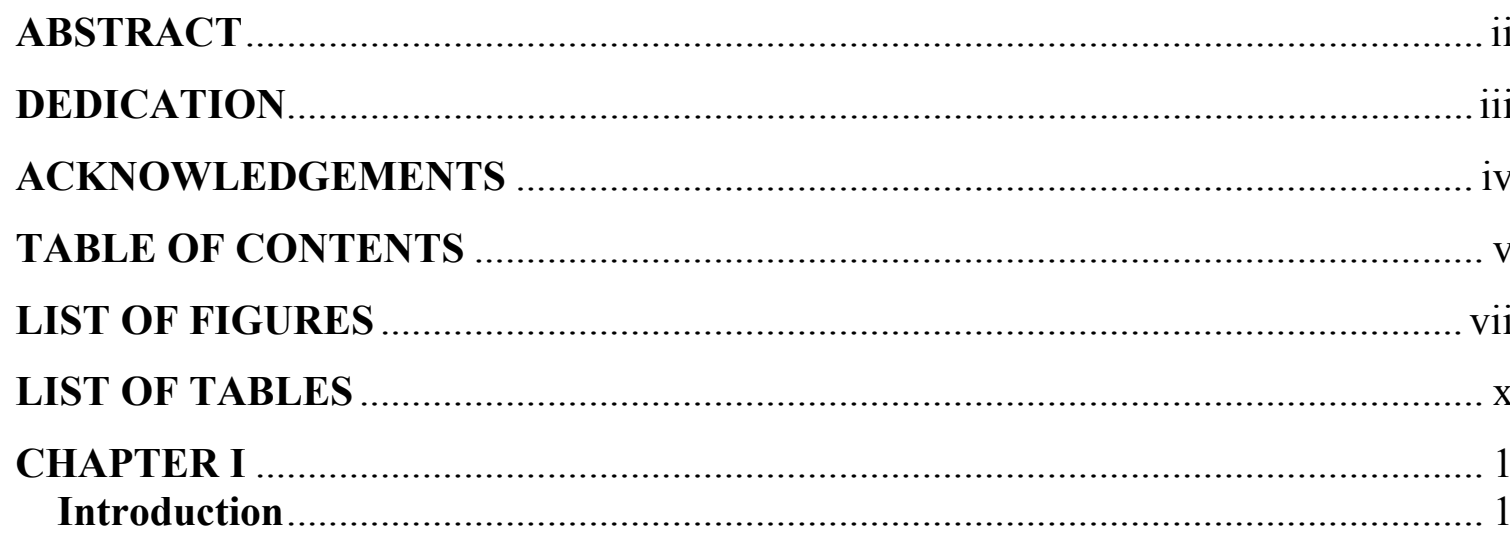

1.1 Bulk Nickel and its Magnetic Properties ........................................................ 1

1.2 Nickel Nanoparticles............................................................................. 1

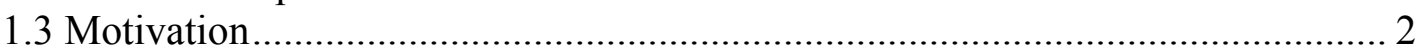

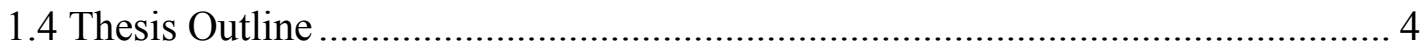

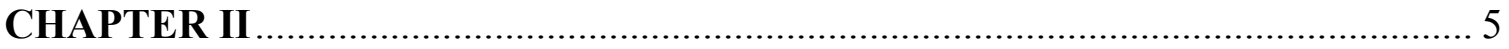

Preparation of Samples and their Characterization............................................ 5

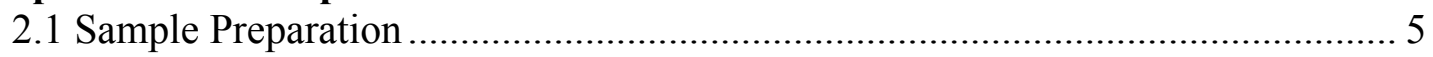

2.2 Thermogravimetric Analysis ......................................................................... 7

2.3 Transmission Electron Microscopy (TEM) ...................................................... 11

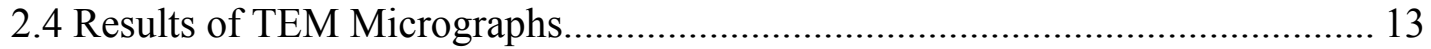

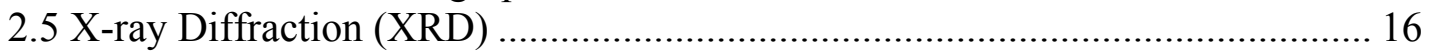

2.6 Structure Factor for Nickel ............................................................................. 22

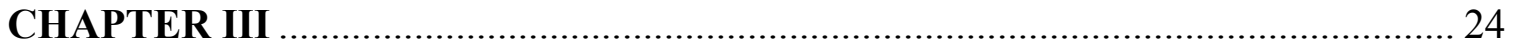

Superparamagnetism: Theoretical Considerations …………………………….... 24

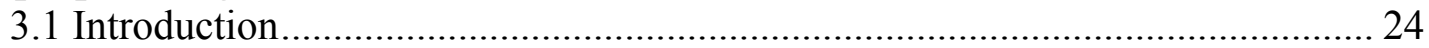

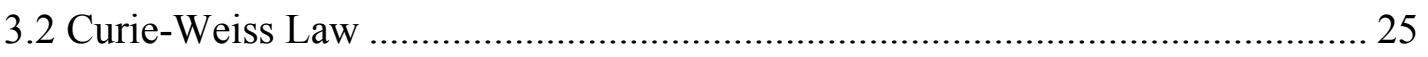

3.3 Magnetization of Non-interacting Magnetic Dipoles ......................................... 25

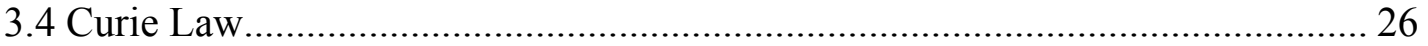

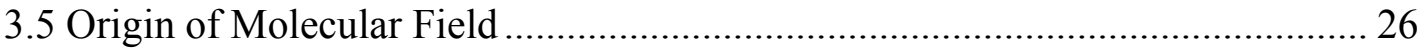

3.6 Relation between Molecular Field and Exchange Constant ............................... 28

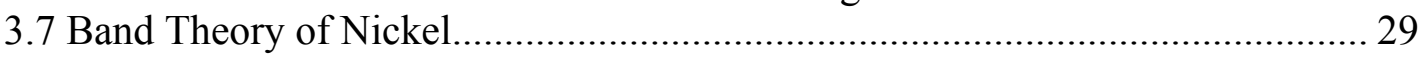

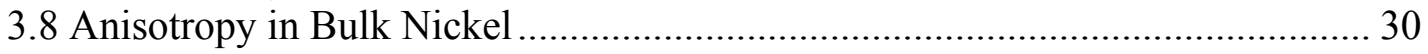

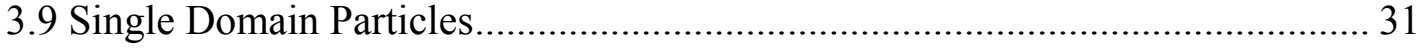

3.10 Variation in Blocking Temperature ............................................................ 33

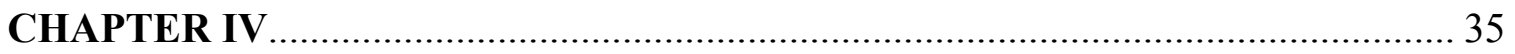

Background Theory on Magnetic Relaxation: Theoretical Considerations.......... 35

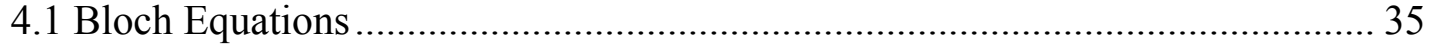

4.2 Magnetic Resonance in Anisotropic Systems .................................................. 37

4.3 AC Susceptibility Theory ………………………............................................. 39 


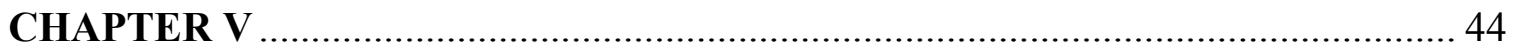

Experimental results from SQUID magnetometry and discussion ...................... 44

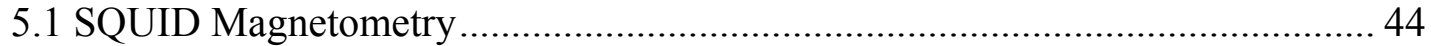

5.2 Blocking Temperature and Particle Size........................................................... 45

5.3 Magnetic Field Dependence of the Blocking Temperature ............................. 47

5.4 Langevin Function and Magnetic Moment per Particle ................................... 51

5.5 Variation of Moment per Particle $\left(\mu_{\mathrm{P}}\right)$ with Particle Size (D) ............................. 53

5.6 Variation of $\mathrm{M}_{\mathrm{o}}$ and $\chi_{\mathrm{a}}$ with Temperature (T) and Particle Size (D) .................. 55

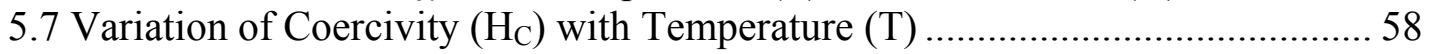

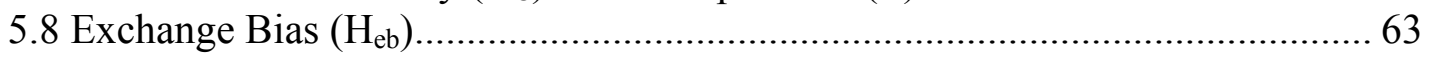

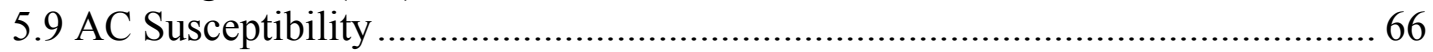

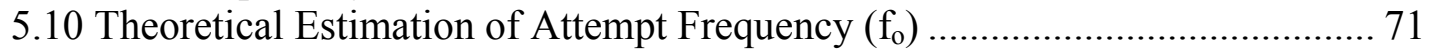

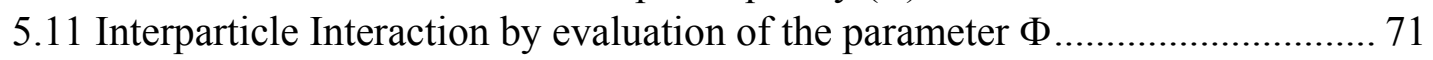

5.12 Theoretical Simulations of AC Susceptibility .............................................. 72

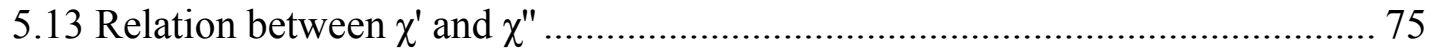

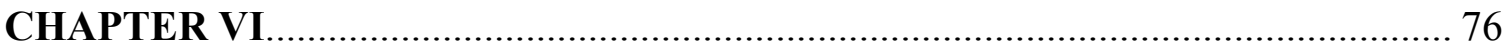

Experimental results from electron magnetic resonance and discussion ............. 76

6.1 Experimental Details............................................................................. 76

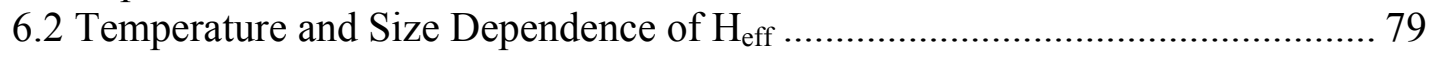

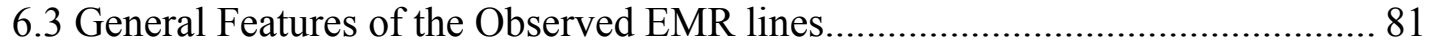

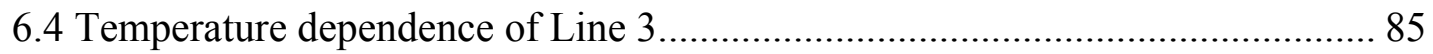

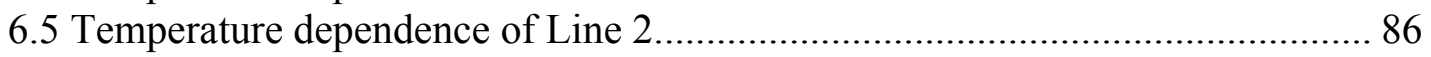

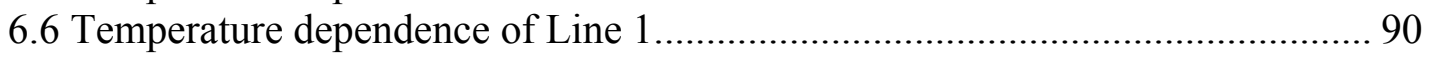

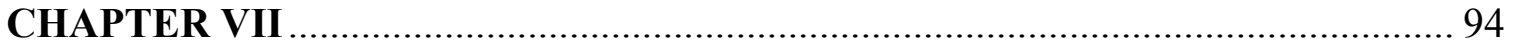

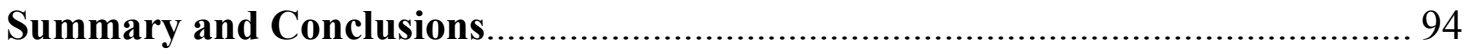

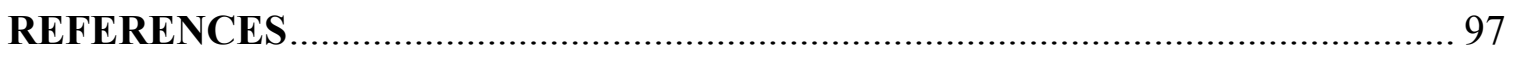

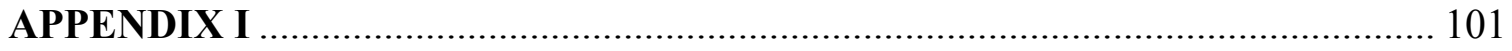

Calculations for Nickel Nanoparticles embedded in Silica matrix..................... 101

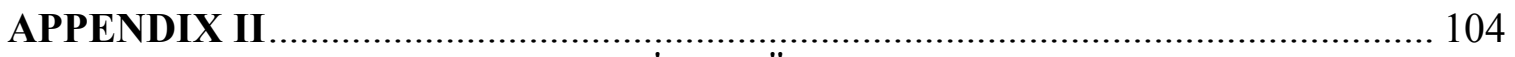

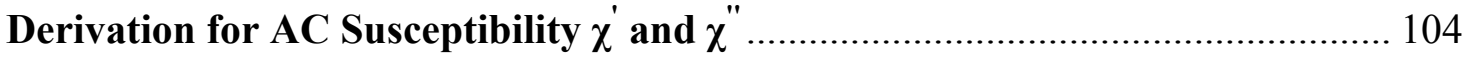

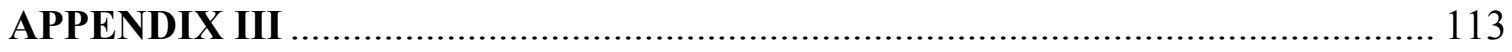

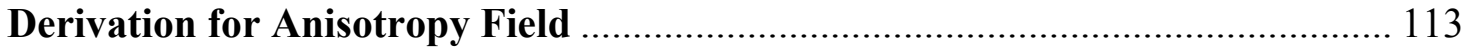




\section{LIST OF FIGURES}

Fig. 2.1 Sequence of steps involved in the preparation of the $15 \% \mathrm{Ni} / \mathrm{SiO}_{2}$ sample.

Fig. 2.2 XRD pattern of $15 \% \mathrm{Ni} / \mathrm{SiO}_{2}$ before and after heating.

Fig. 2.3 Plot mass vs. temperature obtained from TGA showing the decomposition temperature of $367^{\circ} \mathrm{C}$.

Fig. 2.4 Samples annealed in the presence of standard grade $\mathrm{N}_{2}$ showing $\mathrm{Ni} / \mathrm{NiO}$ peaks.

Fig. 2.5 Samples annealed in the presence of UHP $\mathrm{N}_{2}$ showing Ni peaks.

Fig. 2.6 Presence of UHP $\mathrm{N}_{2}$ enhances the formation of the Ni phase.

Fig. 2.7 XRD of $1 \% \mathrm{Ni} / \mathrm{SiO}_{2}$ annealed at different temperatures.

Fig. 2.8 Schematic diagram of a conventional TEM.

Fig. 2.9 DF and BF diagram.

Fig. 2.10 TEM of images of the $15 \% \mathrm{Ni} / \mathrm{SiO}_{2}$ annealed at $\mathrm{T}_{\mathrm{a}}=400^{\circ} \mathrm{C}$ (a) and $\mathrm{T}_{\mathrm{a}}=500^{\circ} \mathrm{C}$ (c) respectively with the log-normal fits to the distribution shown by solid lines in (b) and (d) respectively.

Fig. 2.11 TEM of images of the $15 \% \mathrm{Ni} / \mathrm{SiO}_{2}$ annealed at $\mathrm{T}_{\mathrm{a}}=600^{\circ} \mathrm{C}$ (a) and $\mathrm{T}_{\mathrm{a}}=700^{\circ} \mathrm{C}$ (c) respectively with the log-normal fits shown in (b) and (d) respectively.

Fig. 2.12 TEM of image of the $15 \% \mathrm{Ni} / \mathrm{SiO}_{2}$ annealed at $\mathrm{T}_{\mathrm{a}}=800^{\circ} \mathrm{C}$ is shown in (a) with the log-normal fit shown in (b).

Fig. 2.13 Intensity vs. wavelength for $\mathrm{CuK}_{\alpha}$ lines.

Fig. 2.14 Levels involved in CuK x-ray emission.

Fig. 2.15 Plots of $\beta \cos \theta$ vs. $\sin \theta$ from the Williamson-Hall relation to determine $\mathrm{D}$ and $\eta$ in Eq. 2.9.

Fig. 3.1 Band relationship of Ni above and below the Curie temperature.

Fig. 3.2 Magnetization curve for single crystal Ni [Kittel, 1996].

Fig. 3.3 Ellipsoidal particle with $\mathrm{a}$ and $\mathrm{b}$ as major and minor axis.

Fig. $5.1 \chi$ vs. $\mathrm{T}$ data for 1,5 and $15 \% \mathrm{Ni} / \mathrm{SiO}_{2}$ samples annealed at $\mathrm{T}_{\mathrm{a}}=400$ and $800^{\circ} \mathrm{C}$ in $\mathrm{H}=100$ Oe. The open symbols are for the $\mathrm{FC}$ data and closed symbols are for the ZFC data.

Fig. $5.2 \chi$ vs. T plots for particles sizes 3.8, 11.7, 15, 21 and $23 \mathrm{~nm}$ showing decrease in blocking temperature with an increase in applied field.

Fig. 5.3 Zero field cooled curves $\chi$ vs. T for the applied field varying from $\mathrm{H}=50$ to 
2000 Oe for the $3.8 \mathrm{~nm}$ nanoparticles.

Fig. 5.4 Change in $\mathrm{T}_{\mathrm{B}}$ with applied field $\mathrm{H}$, with the solid lines as fits to Eq. 5.1 for $m=2$ and $m=3 / 2$.

Fig. 5.5 Modified Langevin function fit (Eq. 5.2) done for the $15 \% \mathrm{Ni} / \mathrm{SiO}_{2}$ samples for $\mathrm{D}=3.8,11.7,15,21$ and $23 \mathrm{~nm}$. The inset shows the $\mathrm{M}$ vs. H plots for temperatures above the blocking temperature.

Fig. 5.6 Variation in $\mu_{\mathrm{P}}$ with $\mathrm{D}$ with the solid and dotted line as fits to Eq. 5.10 with $\mu(\mathrm{Ni})=0.606 \mu_{\mathrm{B}}$ and $\mu(\mathrm{Ni})=0.3 \mu_{\mathrm{B}}$ as discussed in text.

Fig. 5.7 $\chi_{\mathrm{a}}$ and $\mathrm{M}_{\mathrm{o}}$ values used to obtain the collapsed curve above the blocking temperature.

Fig. 5.8 M vs. H plots at $\mathrm{T}=5,200 \mathrm{~K}$ with $\mathrm{M}_{\mathrm{o}}$ plotted for $\mathrm{T}=200 \mathrm{~K}$ extrapolated from high field for different $\mathrm{D}$.

Fig. 5.9 Fitting of the measured coercivity against temperature for the $D=3.8 \mathrm{~nm}$ sample. The line is a fit to Eq. 5.14 for $\mathrm{T}_{\mathrm{Bo}}=16 \mathrm{~K}$. The solid curve through the point is for visual aid. $\mathrm{H}_{\mathrm{eb}}$ is exchange bias measured for the sample cooled in $\mathrm{H}=20 \mathrm{kOe}$ to the measuring temperature.

Fig. 5.10 M vs. H plot at $\mathrm{T}=5$ and $200 \mathrm{~K}$ for different particle sizes.

Fig. 5.11 Variation in $\mathrm{H}_{\mathrm{C}}$ with $\mathrm{D}$.

Fig. 5.12 Sample magnetization in a FM/AFM system in applied field from Nogués et al, 1999.

Fig. 5.13 Hysteresis loops measured at $\mathrm{T}=2,4,7 \mathrm{~K}$ for the $\mathrm{D}=3.8 \mathrm{~nm}$ sample cooled in $20 \mathrm{kOe}$.

Fig. 5.14 Temperature variations of the AC susceptibilities $\chi$ ' and $\chi^{\prime \prime}$ for different measuring frequencies, for the five samples investigated in this work.

Fig. 5.15 Plots of $\ln f_{m}$ vs. $T_{B}^{-1}$, the solid line is a least-square fit to yield $f_{o}$.

Fig. 5.16 Plots of $T_{B}$ against $1 / \ln \left(f_{o} / f_{m}\right)$, the solid line is a least-square fit to yield $T_{o}$ and $\mathrm{T}_{\mathrm{a}}$.

Fig. 5.17 Plot of $\mathrm{T}_{\mathrm{a}}$ Vs. D.

Fig. 5.18 Plot of $\mathrm{K}_{1}$ vs. D.

Fig. 5.19 Simulated $\chi^{\prime}$ and $\chi^{\prime \prime}$ for sizes $\mathrm{D}=3.8,11.7,15$ and $21 \mathrm{~nm}$.

Fig. 5.20 Plots of experimental $\chi^{\prime \prime}$ vs. T and computed $\mathrm{C} \partial\left(\chi^{\prime} \mathrm{T}\right) / \partial \mathrm{T}$ using $\chi^{\prime}$ vs. T data of the $\mathrm{D}=3.8,11.7,15$ and $21 \mathrm{~nm}$ samples. 
Fig. 6.1 Variation of anisotropy field with cubic and axial symmetries.

Fig. 6.2 Variation of anisotropy field with temperature for $\mathrm{Ni}$ (cubic) for different particle sizes.

Fig. 6.3 EMR lines for $\mathrm{D}=3.8,11.7,15,21$ and $23 \mathrm{~nm}$ at $\mathrm{T}=5 \mathrm{~K}$.

Fig. 6.4 EMR lines for $\mathrm{D}=3.8 \mathrm{~nm}$ for temperatures $\mathrm{T}=5,10,16,20,31,40,54,68,83$, $93,107,121,133,150,170,190,204,217,240,260,280$ and $294 \mathrm{~K}$.

Fig. 6.5 EMR lines for $\mathrm{D}=11.7 \mathrm{~nm}$ for temperatures $\mathrm{T}=5,9,14,32,66,80,90,114,134$, $150,170,193,215,240,265$ and $280 \mathrm{~K}$.

Fig. 6.6 EMR lines for $\mathrm{D}=15 \mathrm{~nm}$ for temperatures $\mathrm{T}=5,8,14,20,25,29,35,43,48,55$, $75,90,105,115,127,140,155,170,210,250$ and $280 \mathrm{~K}$.

Fig. 6.7 EMR lines for $\mathrm{D}=21 \mathrm{~nm}$ for temperatures $\mathrm{T}=5,5,13,18,28,45,58,70,85,95$, $110,122,132,147,170,190,230$ and $287 \mathrm{~K}$.

Fig. 6.8 EMR lines for $\mathrm{D}=23 \mathrm{~nm}$ for temperatures $\mathrm{T}=5,10,15,20,25,33,40,53,80$, $95,117,130,145,160,180,200,220,235,260$ and $290 \mathrm{~K}$.

Fig.6.9 Peak position of line 3.

Fig. 6.10 Temperature dependence of $\mathrm{H}_{\mathrm{r}}, \Delta \mathrm{H}$ and $\mathrm{I}$ of line 2 for $\mathrm{D}=3.8 \mathrm{~nm}$.

Fig. 6.11 Temperature dependence of $\mathrm{H}_{\mathrm{r}}, \Delta \mathrm{H}$ and $\mathrm{I}$ of line 2 for $\mathrm{D}=11.7 \mathrm{~nm}$.

Fig. 6.12 Temperature dependence of $\mathrm{H}_{\mathrm{r}}, \Delta \mathrm{H}$ and $\mathrm{I}$ of line 2 for $\mathrm{D}=15 \mathrm{~nm}$.

Fig. 6.13 Temperature dependence of $\mathrm{H}_{\mathrm{r}}, \Delta \mathrm{H}$ and $\mathrm{I}$ of line 2 for $\mathrm{D}=21 \mathrm{~nm}$.

Fig. 6.14 Temperature dependence of $\mathrm{H}_{\mathrm{r}}, \Delta \mathrm{H}$ and $\mathrm{I}$ of line 2 for $\mathrm{D}=23 \mathrm{~nm}$.

Fig. 6.15 EMR lines for undoped $\mathrm{SiO}_{2}$ for temperatures $\mathrm{T}=5,20,40,56,70,83,100$, $120,155,195,235,265$ and $285 \mathrm{~K}$.

Fig. 6.16 Comparison of $\mathrm{H}_{\mathrm{r}}, \Delta \mathrm{H}$ and $\mathrm{I}$ for $\mathrm{SiO}_{2}$ and $\mathrm{Ni} / \mathrm{SiO}_{2}\left(\mathrm{~T}_{\mathrm{a}}=400^{\circ} \mathrm{C}\right)$.

Fig. 6.17 Comparison of $\mathrm{SiO}_{2}\left(\mathrm{~T}_{\mathrm{a}}=400^{\circ}\right.$ and $\left.800^{\circ} \mathrm{C}\right)$ signals at room temperature.

Fig.6.18 Gaussian and Lorenzian fits to experimental data for $\mathrm{D}=3.8 \mathrm{~nm}$.

Fig.6.19 Gaussian and Lorenzian fits to experimental data for $\mathrm{SiO}_{2}$. 


\section{LIST OF TABLES}

Table 2.1 Summary of the particle sizes obtained from XRD and TEM.

Table 5.1 Summary of the experimental and simulated values of $T_{B}$ for different frequencies. 


\section{CHAPTER I}

\section{Introduction}

\subsection{Bulk Nickel and its Magnetic Properties}

In a ferromagnetic material the magnetic moments associated with electrons are aligned parallel to each other. This results in spontaneous magnetization even in the absence of an externally applied field. Above the critical temperature denoted as the Curie temperature $\left(\mathrm{T}_{\mathrm{C}}\right)$, the spontaneous magnetization disappears, and the material behaves as a paramagnet with its susceptibility following the Curie Weiss law. The Curie temperature for $\mathrm{Ni}$ is $627 \mathrm{~K}$.

Some ferromagnetic materials do not show any magnetization in zero field but show net magnetization in the presence of a small externally applied field. Weiss explained this phenomena with the presence of domains. Certain materials have randomly oriented domains which lead to the net magnetization of the material to be zero. When

applying an external field, the domains reorient themselves to align along the direction of the applied field and remain there when the field is switched off leading to a net magnetization [Morrish, 2001].

Nickel is a ferromagnetic metal that crystallizes in face centered cubic (FCC) structure with the lattice constant of $3.52 \AA$. The electronic configuration of $\mathrm{Ni}$ is $3 \mathrm{~d}^{8} 4 \mathrm{~s}^{2}$, the partially filled $3 \mathrm{~d}$ shell being responsible for the presence of magnetic properties in $\mathrm{Ni}$. The saturation magnetization for $\mathrm{Ni}$ is $55 \mathrm{emu} / \mathrm{g}$ at room temperature and the effective magnetic moment is $0.606 \mu_{\mathrm{B}} /$ atom [Kittel, 1996]. This fractional moment is explained on the band theory of magnetism.

\subsection{Nickel Nanoparticles}

Domain structures in which the system is in a high energy configuration tend to lower its energy by entering into a domain configuration which has a lower energy. An ideal single domain particle is a fine particle which has its magnetic moment directed towards one end. Single domain particle sizes typically range from 10-100 nm [Kittel, 1996]. The single domain particles have a high magnetic moment and behave as 
paramagnets above a certain temperatures called the blocking temperature $\left(T_{B}\right)$. They are used in digital electronics where their moment can be oriented in a particular direction indicating a 0 or 1 .

Several methods have been used to produce these NPs which include evaporation, sputtering and various chemical methods [Estournès et al, 1997]. The development of hosting metallic NPs in an non-magnetic matrix is an effective way of tailoring a uniform size distribution [Fonseca et al, 2003] and reducing the interaction among NPs. In a noninteracting particle assembly the superparamagnetic relaxation time follows Néel-Brown theory [Néel, 1949]. In assemblies where significant dipole-dipole interactions are present, magnetic relaxation is affected by the interaction. It is also important to know how magnetic dipolar interactions modify the superparamagnetic relaxation rate. There have been models developed predicting how the relaxation rate becomes either faster or slower based on the presence of dipolar interactions [Luis et al, 2002]. For large interparticle distances and small concentration of particles, the dipolar interactions are not so significant [Petracic et al, 2004].

\subsection{Motivation}

It has been known that the magnetic behavior of small sized ferromagnetic materials differ from bulk materials. Since this discovery people have been led in trying to understand the dependence of magnetic properties on particle size. This effect is studied in $\mathrm{Ni} / \mathrm{SiO}_{2}$ system here. $\mathrm{Ni}$ in silica gels is totally reduced to metal NPs and is said to exhibit superparamagnetic behavior. It is stable towards reoxidation when exposed to air at room temperatures. Fe and Co on the other hand do not get completely reduced unless it occurs at higher temperatures $\left(1000^{\circ} \mathrm{C}\right.$ and above) and are said to be less stable towards reoxidation [Lutz et al, 1998]. The purpose of this work is to study the magnetic properties and relaxation effects with particles size of these small isolated Ni NPs embedded in amorphous diamagnetic silica matrix.

Studies have been done on Ni NPs with $\mathrm{D}=5 \mathrm{~nm}$ prepared through laser driven decomposition of $\mathrm{Ni}$ carbonyl vapors and it was observed that the presence of dipolar interactions among the particles were responsible for suppressing the independent character of the particles and the observed blocking temperature for these Ni NPs was 
$150 \mathrm{~K}$ [Sahoo et al, 2005]. By reducing $\mathrm{NiCl}_{2} \cdot 6 \mathrm{H}_{2} \mathrm{O}$ with $\mathrm{NaBH}_{4}$, the Ni NPs prepared were coated with $\mathrm{NiO}$ layer useful in studying antiferromagnetic/ferromagnetic properties [Aparna et al, 2005]. Size control of Ni NPs was also done using reagents such as hexadecylamine and trioctylphosphine to obtain Ni NPs with sizes $D=3-11 \mathrm{~nm}$; however the size distribution was not found to be ideal [Hou et al, 2005].

Ni NPs embedded in silica were successfully prepared by Leite et al [Leite et al, 2002] with particle size ranging from $D=3-41 \mathrm{~nm}$ and $\mathrm{Ni}$ concentration ranging from 5$47 \%$ where they were tested as a catalyst in the formation of $\mathrm{H}_{2}$ gas. In another paper by Leite et al they also prepared $\mathrm{SiO}_{2}$ embedded with $\mathrm{Co}, \mathrm{Ag}$ and $\mathrm{Fe}$ synthesizing them using the same method and they observed that the $\mathrm{Fe} / \mathrm{SiO}_{2}$ specimen showed $\mathrm{Fe}_{3} \mathrm{C}$ [Leite et al, 2002]. Studies done by Fonseca et al on $\mathrm{Ni} / \mathrm{SiO}_{2} \mathrm{NPs}$ with $\mathrm{D} \approx 6 \mathrm{~nm}$ with percent compositions of $1.5 \%$ and $5 \mathrm{wt} \%$ revealed the blocking temperature of $20 \mathrm{~K}$ for the $1.5 \%$ sample and $40 \mathrm{~K}$ for the $5 \%$ sample [Fonseca et al, 2002]. Further work was done on this by Goya et al where they showed the $1.5 \%$ sample with $\mathrm{D}=8.4 \mathrm{~nm}$ followed the NéelArrhenius model for single domain non-interacting particles and the 5\% sample with $\mathrm{D}=6.6 \mathrm{~nm}$ showed an increase in the energy barrier with the additional contribution coming from dipolar interactions [Goya et al, 2003]. Work done by Sharma et al [Sharma et al, 1981] on electron magnetic resonance of $10 \% \mathrm{Ni} / \mathrm{SiO}_{2}$ suggests the line broadening at lower temperatures with the resonance shifting to lower fields.

As noted above, previous studies done on Ni NPs supported by silica matrix have shown superparamagnetic behavior, catalytic activity and effect of dipolar interaction on energy barrier. However systematic size dependence of the magnetic properties of $\mathrm{Ni} / \mathrm{SiO}_{2}$ has been lacking. In this work, an indepth study of $15 \% \mathrm{Ni} / \mathrm{SiO}_{2} \mathrm{NP}$ system for different particles sizes is reported to fill this gap. DC, AC magnetization and electron magnetic resonance (EMR) with varying temperature has been studied. Transmission electron microscopy (TEM) data clearly shows the increase in particle size with in the increase in annealing temperature for all the samples. The $3.8 \mathrm{~nm}$ sample was studied in detail and the DC magnetization data suggested that the blocking temperature decrease with the applied field $\mathrm{H}$ follows the $\mathrm{H}^{2}$ dependence for lower fields and $\mathrm{H}^{3 / 2}$ dependence for higher fields. For all the different sizes the value of the magnetic moment per particle increases with increase in particle size. AC susceptibility studies for frequencies $0.1,1$, 
99, 499 and $997 \mathrm{~Hz}$ clearly shows that with increase in particle size, the possibility of interparticle interaction increases hence affecting the attempt frequency. EMR showed the appearance of a very sharp line near $\mathrm{H}=3300$ Oe which is identified with the defects in $\mathrm{SiO}_{2}$ matrix. The other two lines are from the Ni NPs. It is hoped that the following work would create interest leading to further interest in studying the experimental and theoretical aspects of such systems.

\subsection{Thesis Outline}

Details of steps taken to prepare the samples under certain conditions are discussed in chapter II followed by thermogravimetric experiments done to obtain starting conditions and leading to obtaining the particle size from TEM and XRD analysis. Chapter III summarizes the theory behind magnetism based on Curie law followed by discussion of single domain particles and their blocking temperatures. Chapter IV deals with Bloch equations formalism of magnetic resonance and the study of relaxation effects arising from $\mathrm{AC}$ susceptibility measurements. Chapter $\mathrm{V}$ is an experimental section that deals with the $\mathrm{DC}$ and $\mathrm{AC}$ measurements obtained using the superconducting quantum interference device (SQUID) magnetometer. Results on the variation of blocking temperature with applied field, particle size, AC measuring frequency and the effect of interparticle interaction are presented in this chapter. In Chapter VI, temperature dependence of the EMR spectra for various particle sizes are presented and the results are discussed in terms of the temperature and size induced anisotropy of the superparamagnetic particles. In Chapter VII, a summary of the major results of this dissertation are presented, followed by suggestions for future studies. Various appendices are given at the end of the thesis. 


\section{CHAPTER II}

\section{Preparation of Samples and their Characterization}

This chapter covers the preparation of the $\mathrm{Ni} / \mathrm{SiO}_{2}$ samples via the sol-gel technique. The samples are annealed under different conditions to obtain pure Ni NPs embedded in $\mathrm{SiO}_{2}$ matrix. Thermogravimetric analysis (TGA) followed by x-ray diffraction (XRD) was performed on these samples in order to determine the starting conditions and the formation of these nanoparticles without contamination. The sizes of these nanoparticles were determined using transmission electron microscopy (TEM) and their distribution. The sizes are then compared with the sizes determined from XRD using the Debye Scherrer relation.

\subsection{Sample Preparation}

The Ni nanoparticles (NPs) dispersed in $\mathrm{SiO}_{2}$ matrix were synthesized using the sol-gel technique following the work by Leite et al [Leite et al, 2002]. The starting compounds were $\mathrm{Ni}\left(\mathrm{NO}_{3}\right)_{2} \cdot 6 \mathrm{H}_{2} \mathrm{O}$ (Alfa Aesar 99.9985\%), tetraethylorthosilicate (TEOS) (Alfa Aesar 99.9\%), citric acid anhydrous (Fisher Scientific) and ethylene glycol anhydrous (Sigma Aldrich 99.8\%) mixed in appropriate amounts. Ni content in the samples was taken to be 1, 5 and $15 \%$. The medium for the mixture was ethanol. Citric acid was dissolved in ethanol along with TEOS and stirred to prepare a homogeneous mixture. The Ni content was added to the mixture of citric acid/metal $(\mathrm{Ni}+\mathrm{Si})$ in the ratio of 3:1 in moles, followed by ethylene glycol being added at a mass ratio of 40:60 to citric acid. The solution was then allowed to dry and form a resin at about $80^{\circ} \mathrm{C}$ in the Fisher Isotemp Oven for several hours. The resin was then heated at $300^{\circ} \mathrm{C}$ for 6 hours and a solid residue was formed which was grinded using a mortar and pestle. The ground sample was then reheated in the presence of ultra high pure (UHP) $\mathrm{N}_{2}$ for 2 hours at temperatures of $400^{\circ}, 500^{\circ}, 600^{\circ}, 700^{\circ}$ and $800^{\circ} \mathrm{C}$. The details for the calculations are presented in appendix I. The following reaction takes place, where citric acid chelates (combines with a metal in complexes in which the metal is part of a ring) $\mathrm{Si}$ and $\mathrm{Ni}$, and 
ethylene glycol is added to promote the polyesterification (a complex ester used in making resin) [Leite et al, 2002]:

Nickel Nitrate + Citric acid $\rightarrow$ Nickel Oxalosuccinate + Nitric acid

$\mathrm{Ni}\left(\mathrm{NO}_{3}\right)_{2}+\mathrm{C}_{6} \mathrm{H}_{8} \mathrm{O}_{7} \rightarrow \mathrm{Ni}\left(\mathrm{C}_{6} \mathrm{H}_{6} \mathrm{O}_{7}\right)+2 \mathrm{HNO}_{3}$

Tetraethoxyorthosilicate + Citric acid $\rightarrow$ Si-Citrate + Ethyl Alcohol

$\left(\mathrm{C}_{2} \mathrm{H}_{5}\right)_{4} \mathrm{SiO}_{4}+2 \mathrm{C}_{6} \mathrm{H}_{8} \mathrm{O}_{7} \rightarrow \mathrm{Si}\left\{[\mathrm{COH}(\mathrm{COOH})]\left[\mathrm{CH}_{2}(\mathrm{COO})\right]_{2}\right\}_{2}+4 \mathrm{C}_{2} \mathrm{H}_{5} \mathrm{OH}$

1 gm of nickel nitrate
+
4gm TEOS
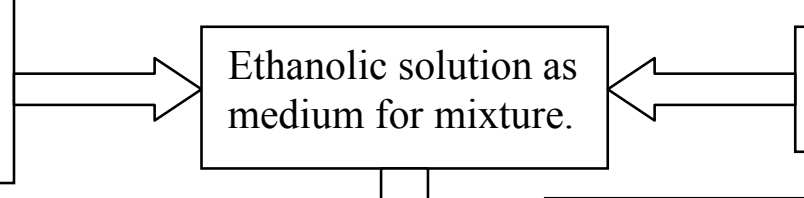

$11.53 \mathrm{gm}$ of citric acid added

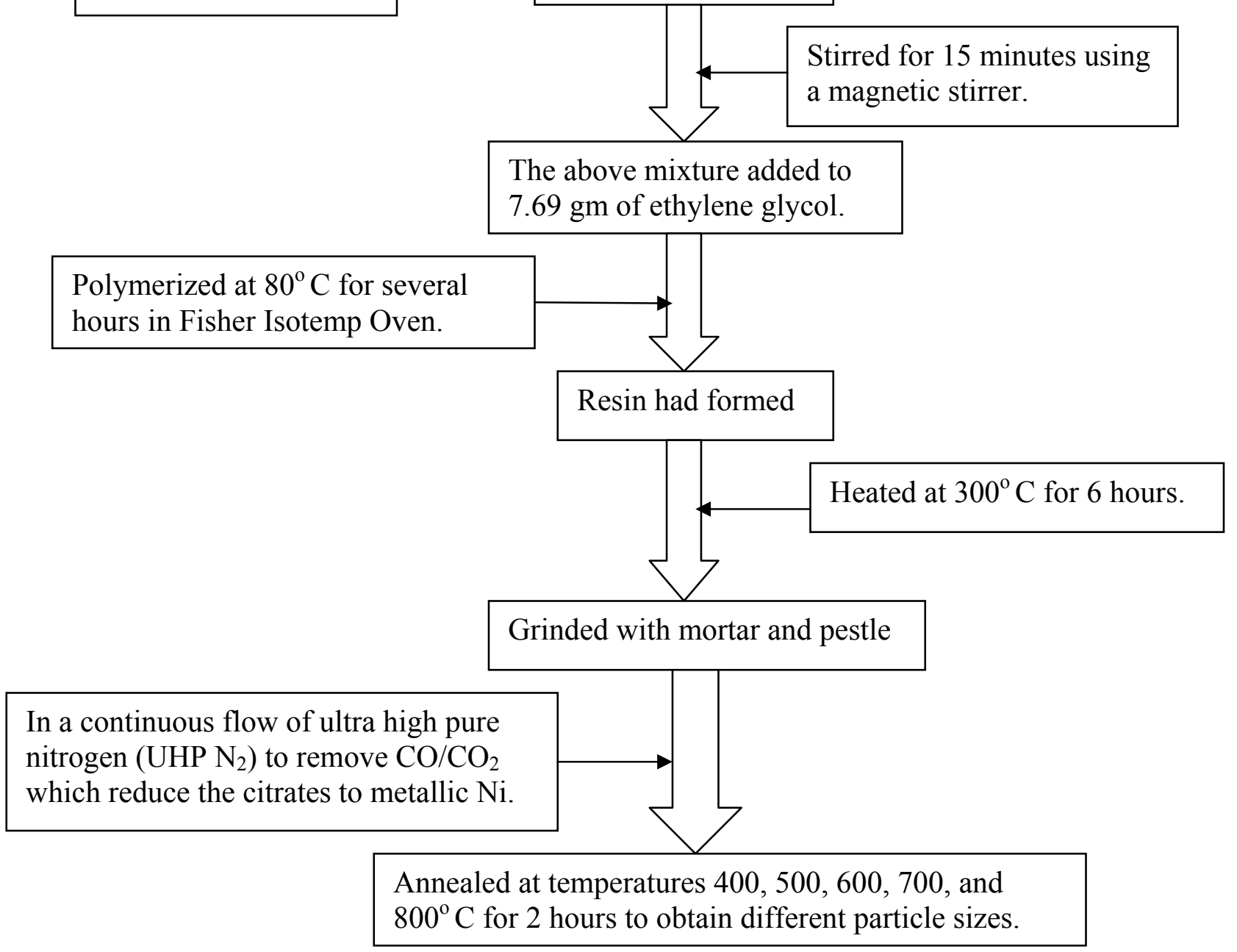

Fig. 2.1 Sequence of steps involved in the preparation of the $15 \% \mathrm{Ni} / \mathrm{SiO}_{2}$ sample. 


\subsection{Thermogravimetric Analysis}

In order to determine the temperature at which the Ni NPs are formed, TGA measurements were done. These measurements were performed by placing the sample on the pan balance which was further inserted into a furnace and heated in air. The conditions for doing the TGA were that the furnace chamber temperature was varied from $30^{\circ} \mathrm{C}-900^{\circ} \mathrm{C}$ at a heating rate of $10^{\circ} \mathrm{C} / \mathrm{min}$. Mass loss as a function of temperature was measured followed by obtaining the XRD pattern on the remaining sample residue. XRD patterns in Fig. 2.2 show that the precursor shows no signs of Ni NPs present. However when the precursor undergoes heating from $30^{\circ} \mathrm{C}-900^{\circ} \mathrm{C}$ in air, the formation of $\mathrm{NiO}$ takes place.

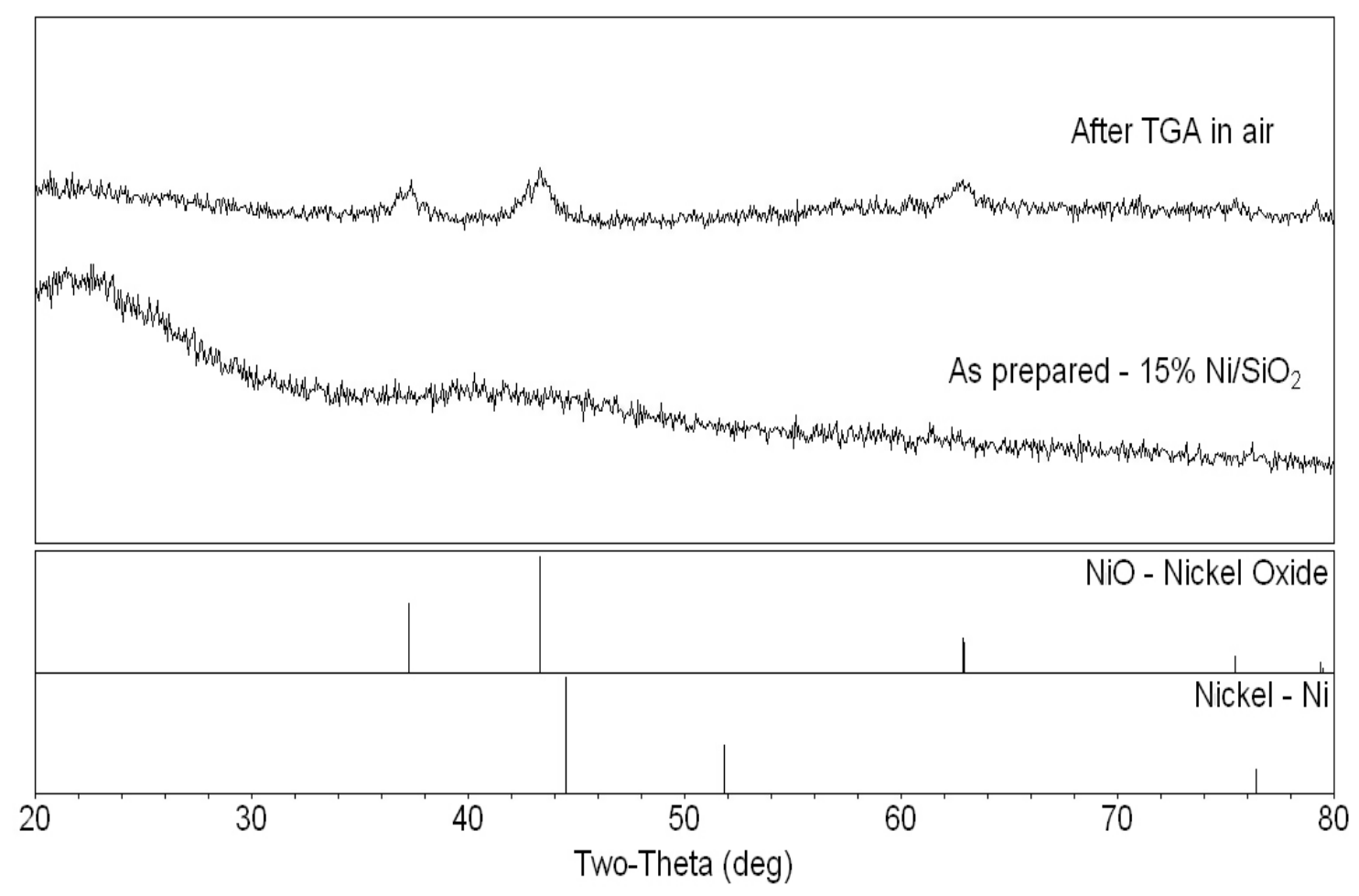

Fig. 2.2 XRD pattern of $15 \% \mathrm{Ni} / \mathrm{SiO}_{2}$ before and after heating. 
The weight loss from Eq. 2.3 below was calculated as $80 \%$ for the sample which initially consisted of Ni-oxalosuccinate Eq. 2.1 and Si-citrate complex Eq. 2.2 that decomposed to give $\mathrm{Ni}-\mathrm{NiO} / \mathrm{SiO}_{2}$. The decomposition temperature was obtained from the derivative of the mass vs. temperature curve and was found to be at $\mathrm{T}=367^{\circ} \mathrm{C}$.

The samples were annealed at temperatures $250^{\circ}, 400^{\circ}, 600^{\circ}$ and $800^{\circ} \mathrm{C}$ in the presence of standard grade nitrogen gas $\mathrm{N}_{2}$ for a period of 2 hours and the XRD patterns show that the formation of the $\mathrm{Ni} / \mathrm{NiO}$ phase does not take place for temperatures less than $400^{\circ} \mathrm{C}$ in Fig. 2.4. Even at $400^{\circ} \mathrm{C}$, the Ni peaks are not so prominent. Assuming that the $\mathrm{NiO}$ is being formed from the oxygen present in standard grade $\mathrm{N}_{2}$ gas, we next annealed the samples in ultra high pure (UHP) $\mathrm{N}_{2}$ gas. The XRD patterns of Fig. 2.5 confirm the absence of any $\mathrm{NiO}$ and formation of only Ni NPs. UHP $\mathrm{N}_{2}$ is more promising than $\mathrm{N}_{2}$ because it prevents the formation of $\mathrm{NiO}$ and it helps in the formation of the Ni phase at a lower temperature as it can be seen from the XRD of $5 \% \mathrm{Ni} / \mathrm{SiO}_{2}$ in Fig. 2.6. XRD pattern of the $1 \% \mathrm{Ni} / \mathrm{SiO}_{2}$ Fig. 2.7 sample shows only the single strong peak of $\mathrm{Ni}$ at higher temperatures only possibly because of the small concentration of $\mathrm{Ni}$.

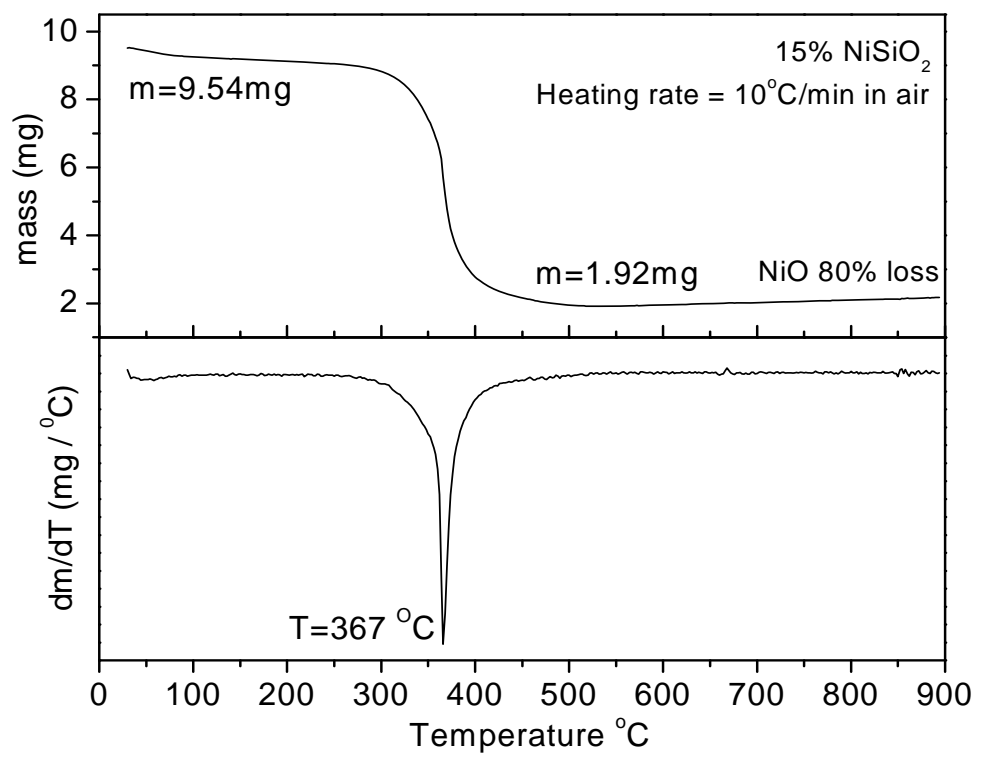

Fig. 2.3 Plot mass vs. temperature obtained from TGA showing the decomposition temperature of $367^{\circ} \mathrm{C}$.

$$
\text { Weight Loss }=\left(\frac{9.54-1.92}{9.54}\right) \times 100=80 \%
$$




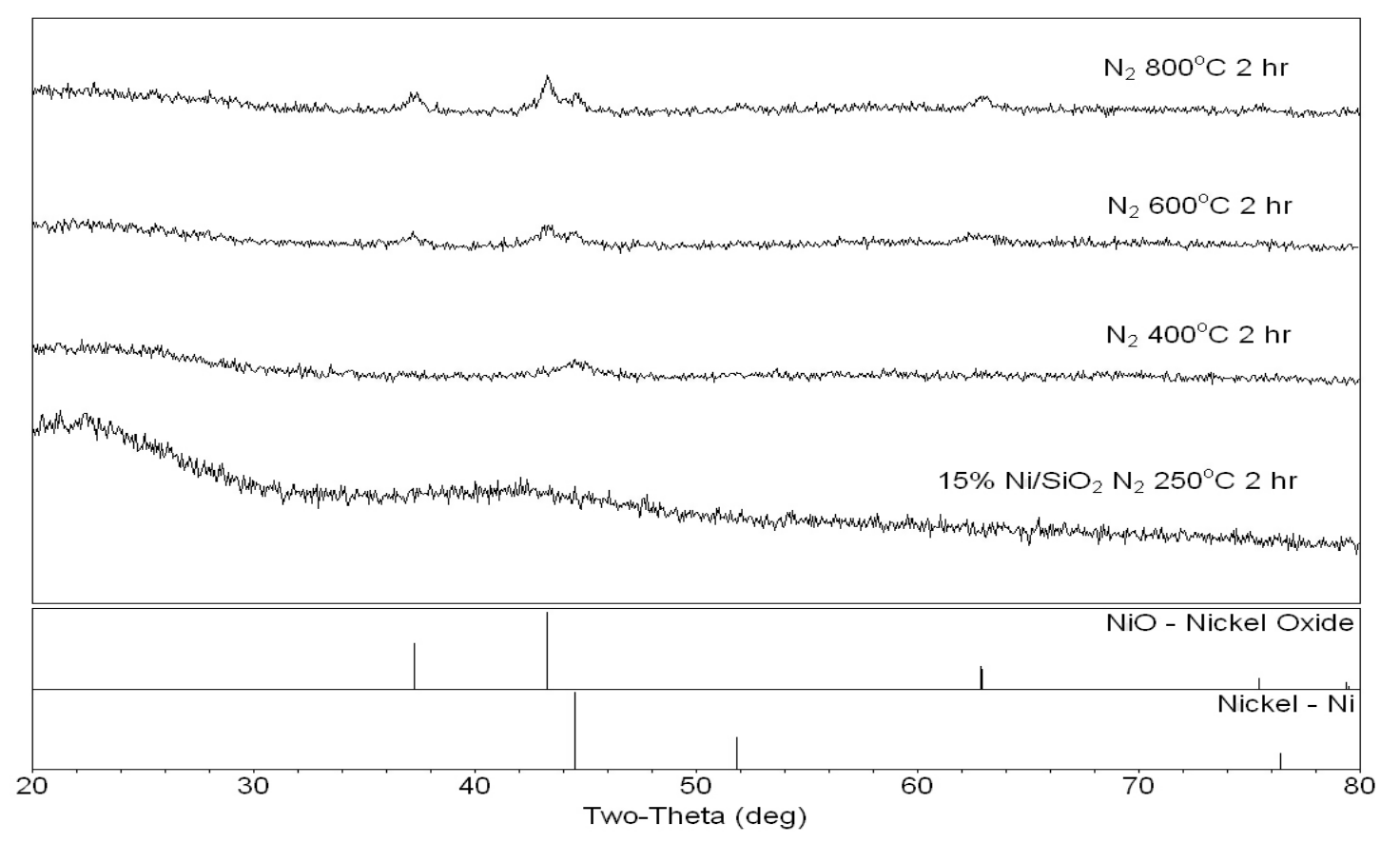

Fig. 2.4 Samples annealed in the presence of standard grade $\mathrm{N}_{2}$ showing $\mathrm{Ni} / \mathrm{NiO}$ peaks.

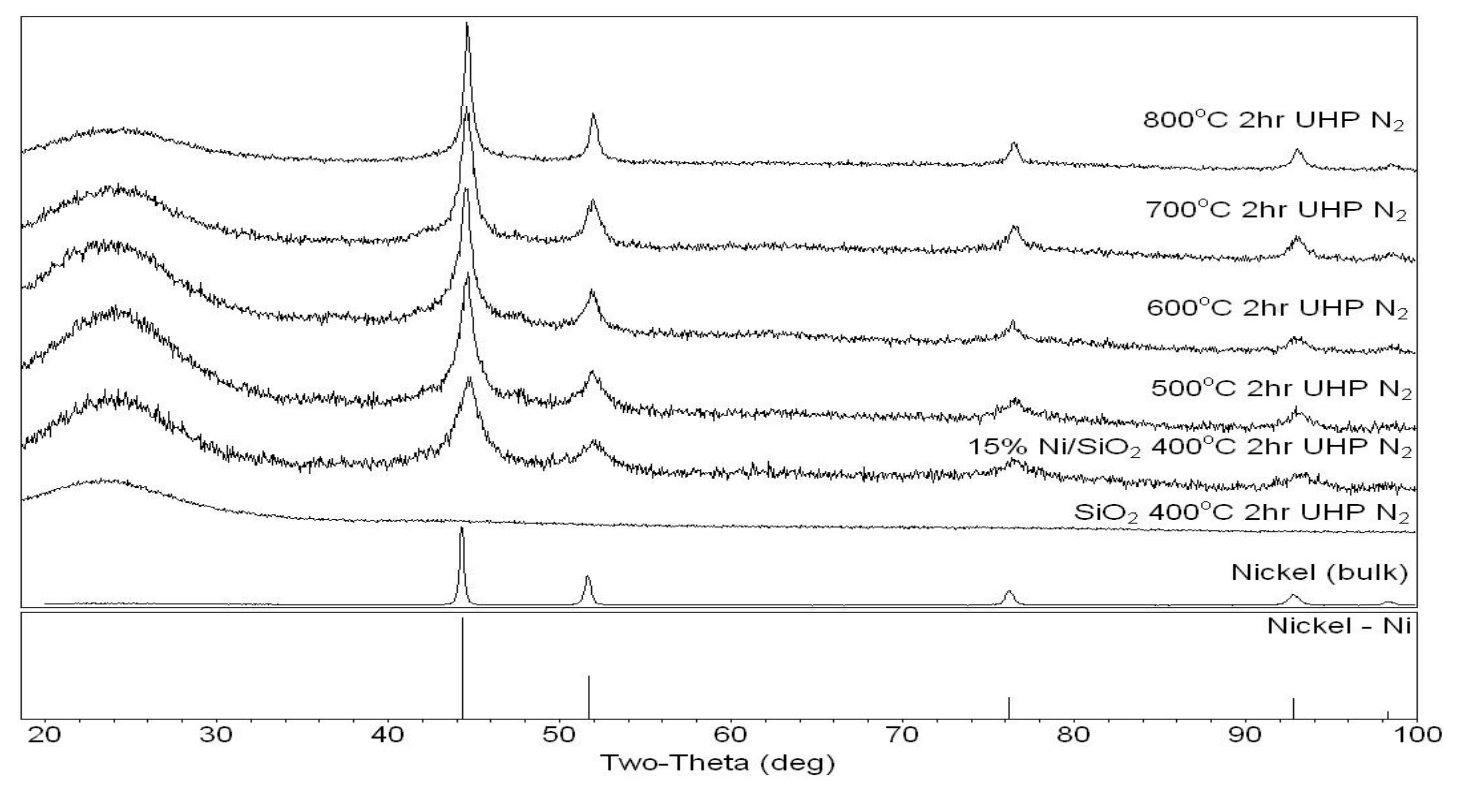

Fig. 2.5 Samples annealed in the presence of UHP $\mathrm{N}_{2}$ showing Ni peaks. 


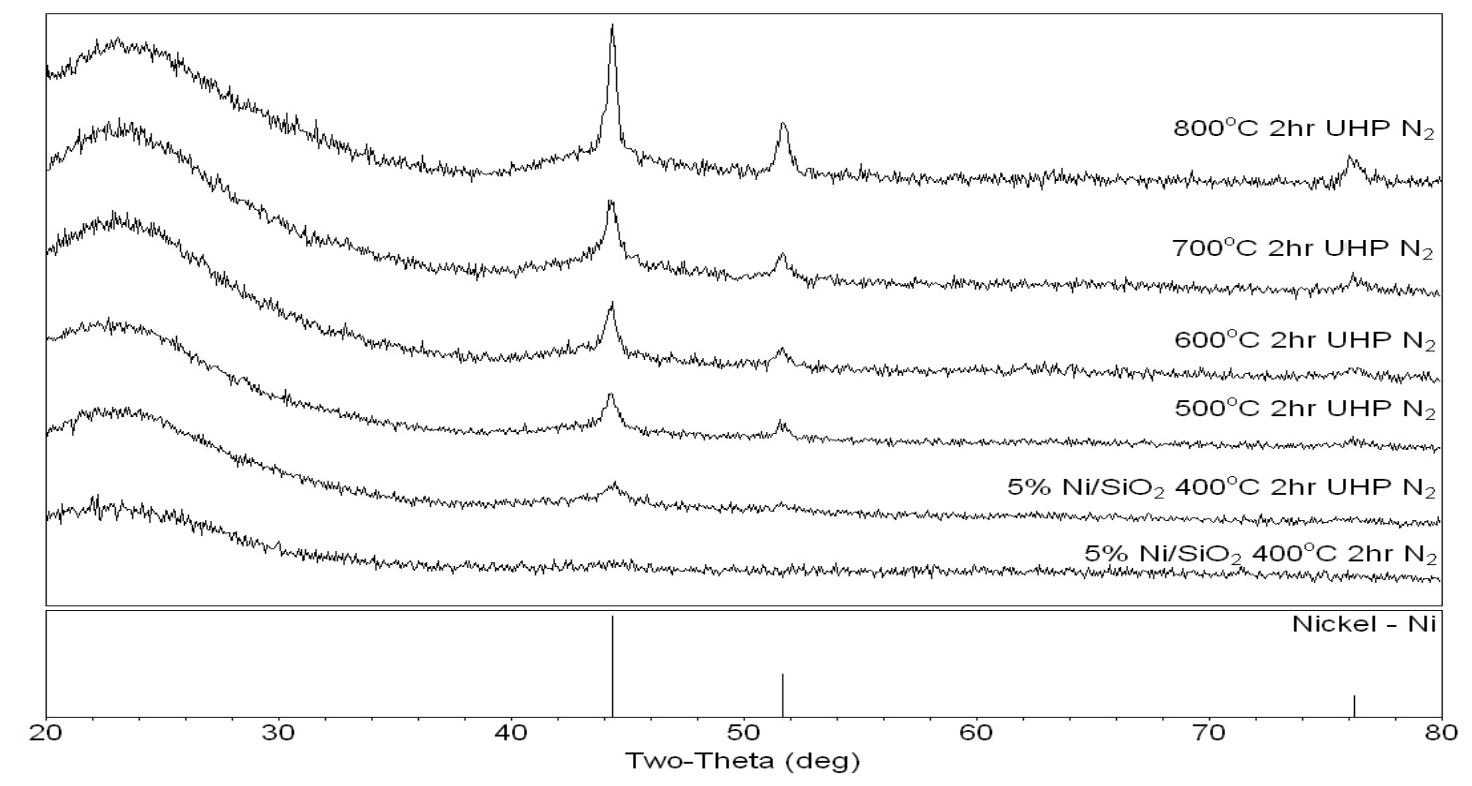

Fig. 2.6 Presence of UHP $\mathrm{N}_{2}$ enhances the formation of the Ni phase.

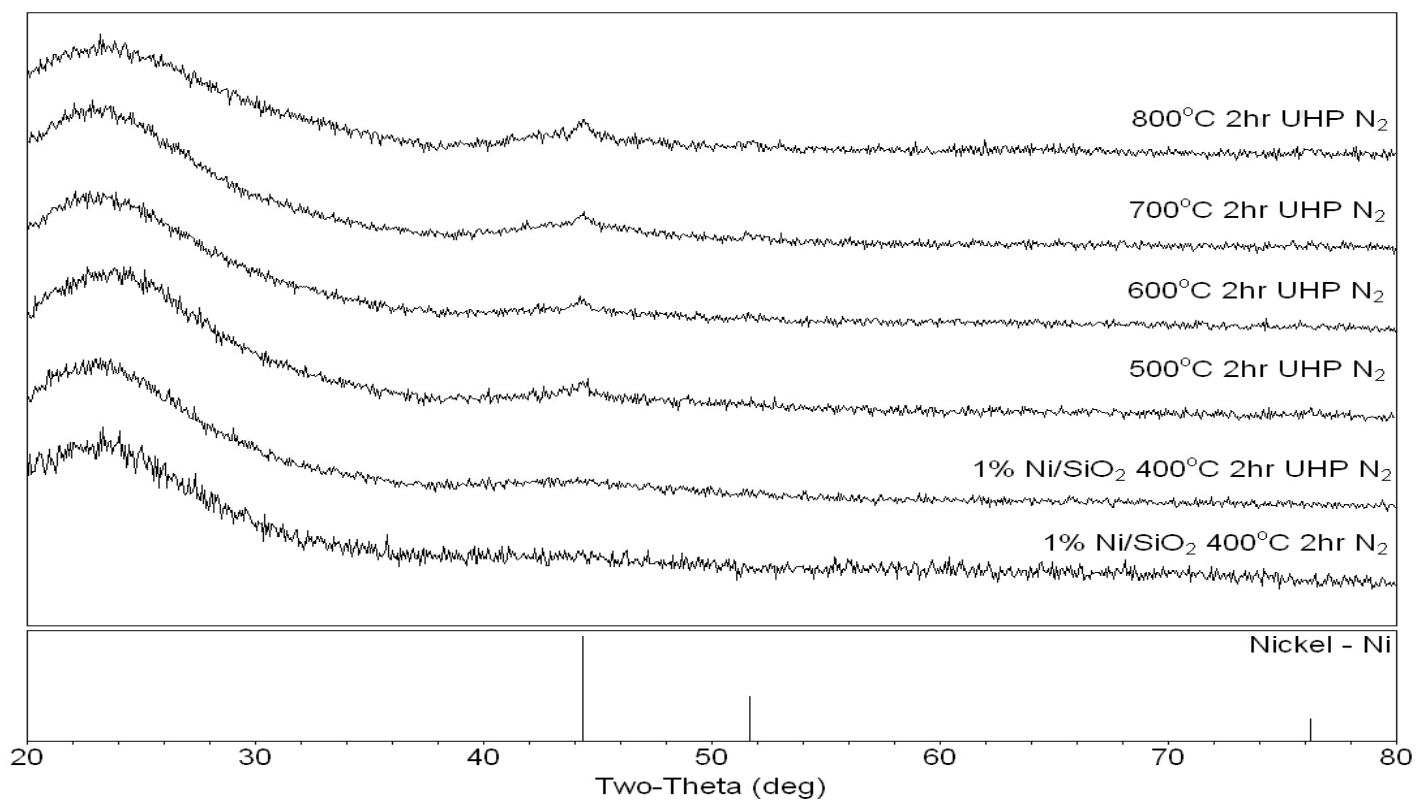

Fig. 2.7 XRD of $1 \% \mathrm{Ni} / \mathrm{SiO}_{2}$ annealed at different temperatures. 
An important process that is undergoing during the decomposition of $\mathrm{Ni}$ oxalosuccinate and Si-citrate is the Mond Process where the purification of Ni takes place through the reaction:

$$
\mathrm{Ni}+4 \mathrm{CO} \rightarrow \mathrm{Ni}(\mathrm{CO})_{4} \rightarrow \mathrm{Ni}+4 \mathrm{CO}
$$

$\mathrm{CO}$ is said to be highly reactive with $\mathrm{Ni}$ in that it can etch the surface within a couple of minutes. After the $\mathrm{CO}$ helps in the purification of $\mathrm{Ni}$ left behind from the $\mathrm{Ni}$ tetracarbonyl which decomposes at $230^{\circ} \mathrm{C}$ [Winter, 2007], it is flushed with the help of UHP $\mathrm{N}_{2}$ flowing through the chamber to prevent the oxidation of $\mathrm{Ni}$.

\subsection{Transmission Electron Microscopy (TEM)}

In a conventional TEM [e.g. see Poole et al, 2003], a beam of electrons emitted from a source are focused through the electromagnetic lens and are limited by an aperture (AP) before they enter the specimen. Upon entering the specimen they get scattered as they pass through it. The electrons after passing through the specimen are focused by an objective lens (the first lens that receives electrons passing through the sample being examined and forms its image). They are then amplified by a magnifying projector (a device for projecting the image onto the screen) to finally produce the image. The image could be formed on a fluorescent screen or a photographic plate.

The selected area electron diffraction (SAED) AP is there to select the transmitted electron beam from a particular area of the specimen. This is done by blocking the beam except for a small fraction passing through one of the holes by moving the AP hole to the section of the specimen the user wants to examine. This beam also contains electrons that have lost energy in inelastic scattering with no deviation in their path of travel and that have been reflected by various (hkl) planes. With the aperture inserted along the path of the undeviated beam a bright- field (BF) image is obtained. When the aperture is adjusted to view only one of the reflected beam from a particular (hkl) plane, then the dark-field 
(DF) image is obtained. So the purpose of the SAED is to allow one to view the diffraction pattern from a particular region of the sample. The BF represents areas where more electrons were transmitted through and DF represents areas where less electrons are transmitted through.

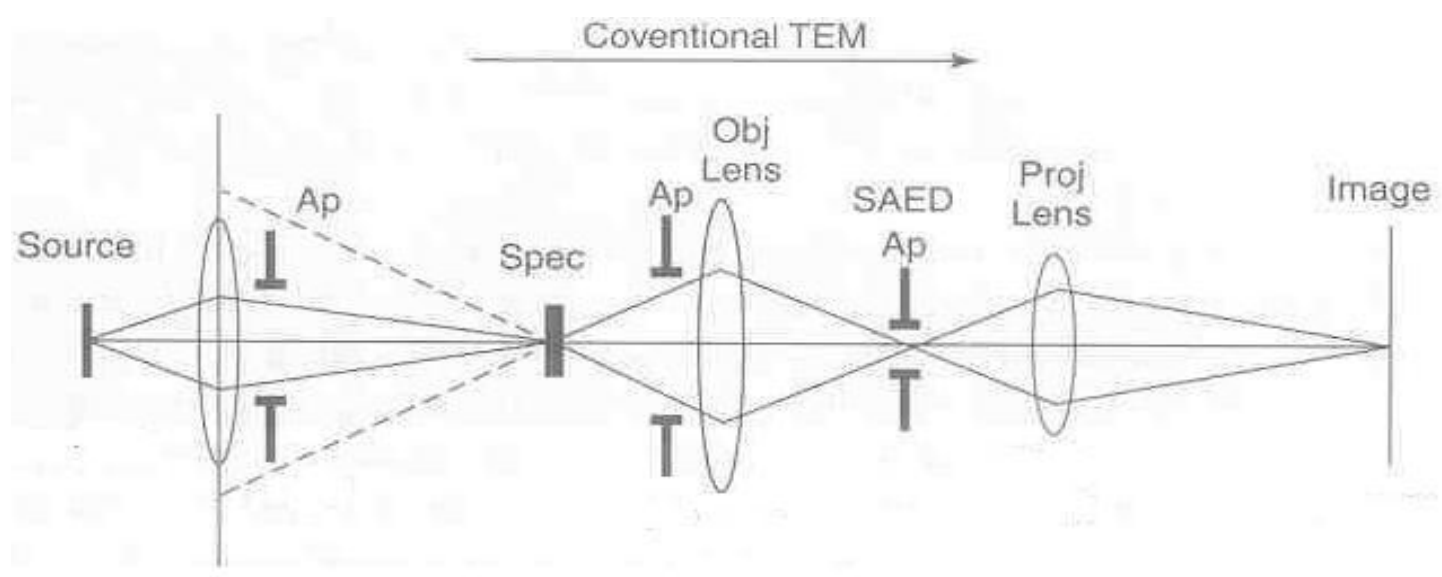

Fig. 2.8 Schematic diagram of a conventional TEM.

For elastic scattering of $100 \mathrm{keV}$, average distance travelled (mean free path) between scattering events varies from a few nanometers to hundreds of nanometers depending on the size of the scatterer. For good images to be obtained, the thickness of the film has to be of the order of the mean free path. Thinner films exhibit too little scattering and thicker ones dominate in multiple scattering making the image blurred. Information collected from the electrons losing energy through other processes such as induced vibrational motion of atoms near the path of an electron creating phonons, or the incoming electron inducing an electron excitation in an atom leading to a transition within the energy levels of the atom is used in other areas of spectroscopy.

The wavelength for incident electrons is given by:

$$
\lambda=\frac{h}{p}=\frac{h}{\sqrt{2 m E}}=\frac{6.626 \times 10^{-34}}{\sqrt{2 \times 9.1 \times 10^{-31} \times 1.6 \times 10^{-19}} \sqrt{V}}=\frac{0.0388 \mathrm{~nm}}{\sqrt{V(\mathrm{kV})}}
$$

So for an accelerating voltage of $100 \mathrm{kV}$, the wavelength of the high energy electrons is a fraction of a nanometer and the spacing between the atoms is only slightly larger. So the atoms act as a diffraction grating to the electrons which are diffracted. 


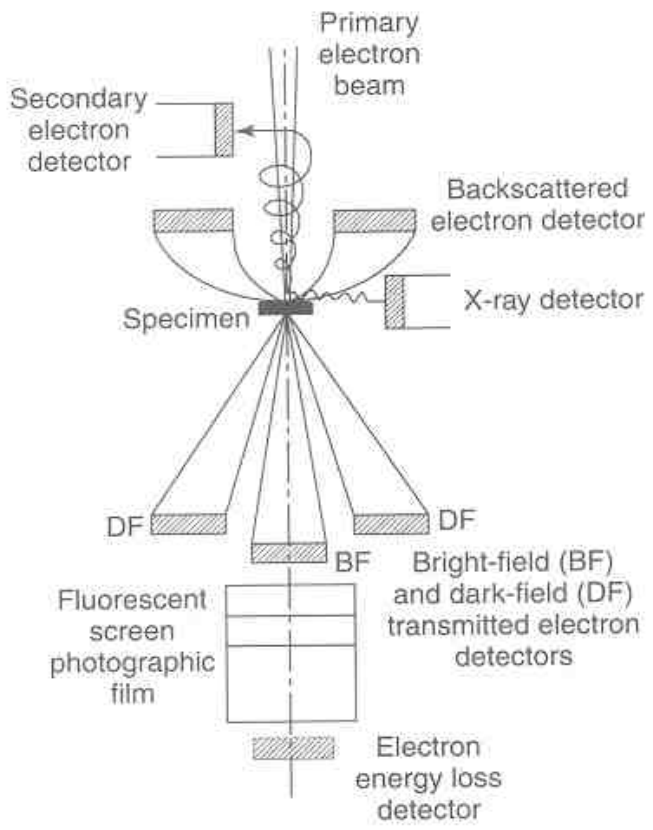

Fig. 2.9 DF and BF diagram

\subsection{Results of TEM Micrographs}

TEM micrographs were done on the $15 \% \mathrm{Ni} / \mathrm{SiO}_{2}$ nanoparticles by Dr. John Bonevich of NIST (National Institute of Standards and Technology, Gaithersburg MD). TEM images provide us with the information on the size of the Ni NPs and their morphology. The figures below show that the Ni NPs are embedded in silica matrix, and their shapes are approximately spherical. The histograms depicting the size distribution of sizes were obtained by measuring the sizes of about 50 particles using a travelling microscope. These histograms were fitted to the log-normal distribution [e.g. see Fonseca et al, 2002] given by:

$$
f(D)=\frac{1}{\sigma D \sqrt{2 \pi}} e^{-\frac{\ln ^{2}\left(D / D_{o}\right)}{2 \sigma^{2}}}
$$

Here $\mathrm{D}$ is the particle diameter, $\mathrm{D}_{\mathrm{o}}$ is the mean diameter of the particle and $\sigma$ is the standard deviation.

Fig. 2.10a shows the results of the TEM measurements on the $15 \% \mathrm{Ni} / \mathrm{SiO}_{2}$ sample annealed at $\mathrm{T}_{\mathrm{a}}=400^{\circ} \mathrm{C}$. The log-normal distribution fit to the histogram shows the 
average particle size of the particles to be $3.8 \mathrm{~nm}$ in Fig 2.10b. The dark spots are due to Ni NP embedded in the silica matrix, and the shape of the particles appears to be approximately spherical. Similar results for samples annealed at $T_{a}=500^{\circ}, 600^{\circ}, 700^{\circ}$ and $800^{\circ} \mathrm{C}$ are given in Fig. 2.10, Fig. 2.11 and Fig. 2.12 respectively. It is evident that increase in the annealing temperature increases the average particle size as expected from Oswald ripening. In Oswald ripening, the smaller particles coalesce to produce larger particles due to increase in the available thermal energy. The average size of the sample annealed at $T_{a}=800^{\circ} \mathrm{C}$ is $23 \mathrm{~nm}$ in Fig. 2.12 .

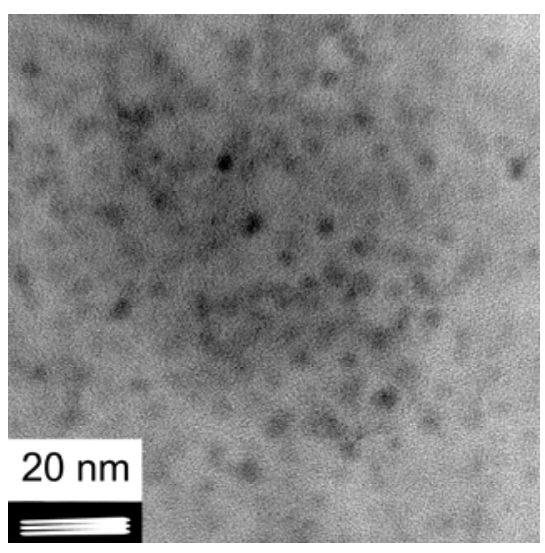

(a)

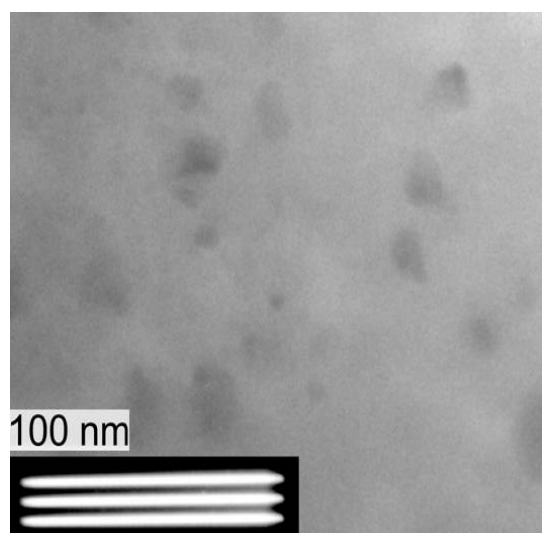

(c)

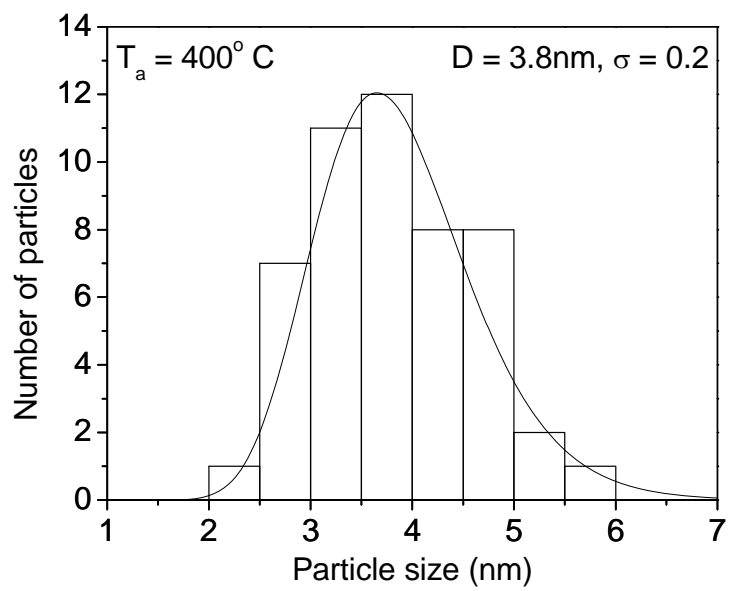

(b)

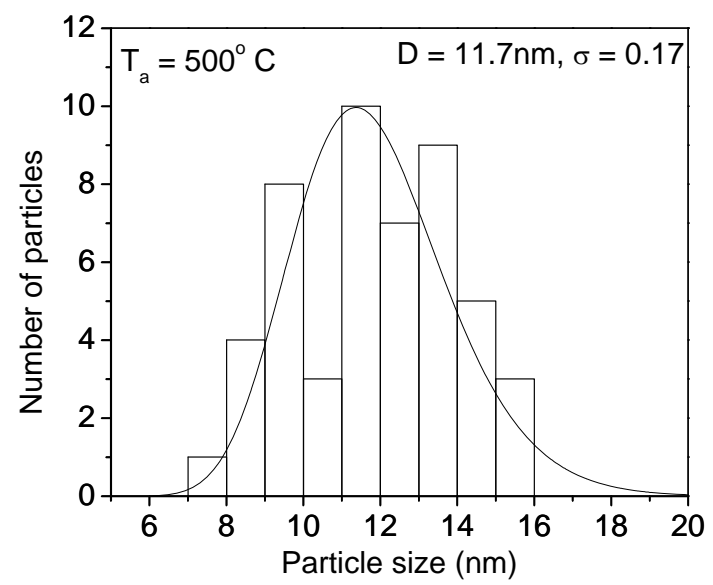

(d)

Fig. 2.10 TEM of images of the $15 \% \mathrm{Ni} / \mathrm{SiO}_{2}$ annealed at $\mathrm{T}_{\mathrm{a}}=400^{\circ} \mathrm{C}$ (a) and $\mathrm{T}_{\mathrm{a}}=500^{\circ} \mathrm{C}$ (c) respectively with the log-normal fits to the distribution shown by solid lines in (b) and (d) respectively. 


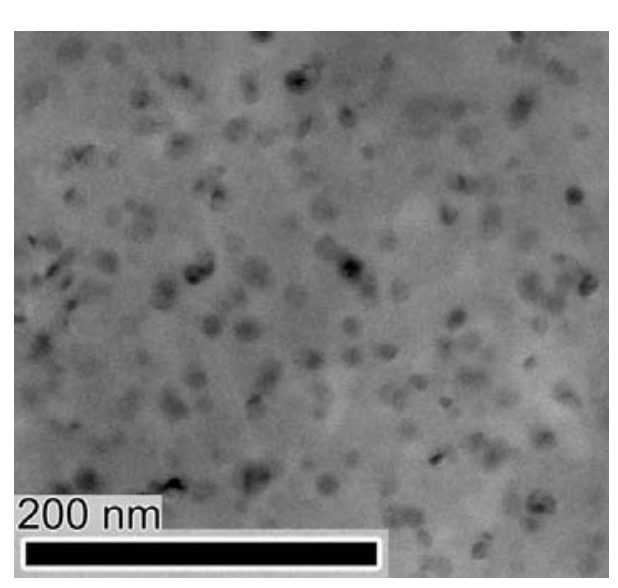

(a)

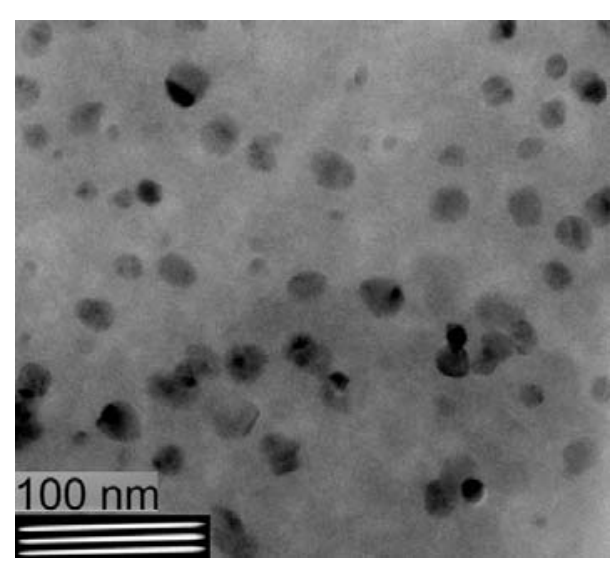

(c)

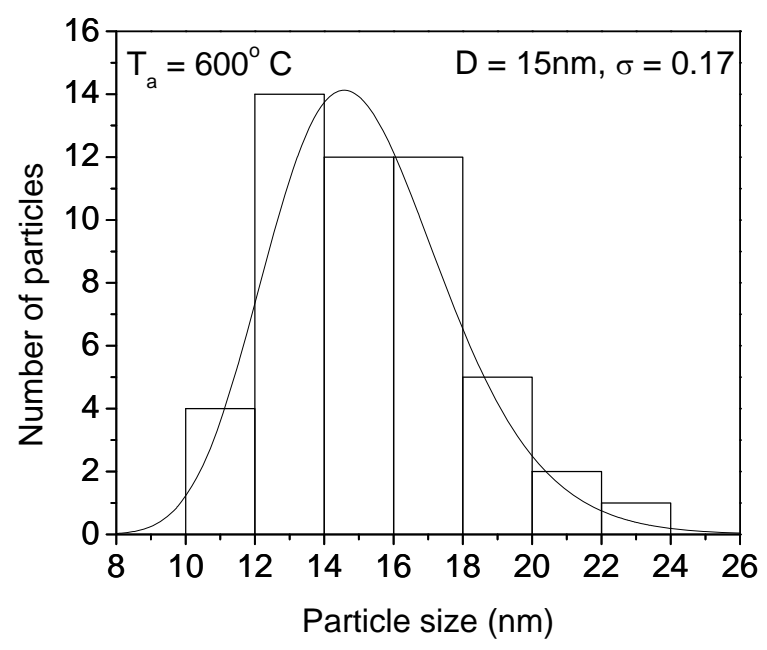

(b)

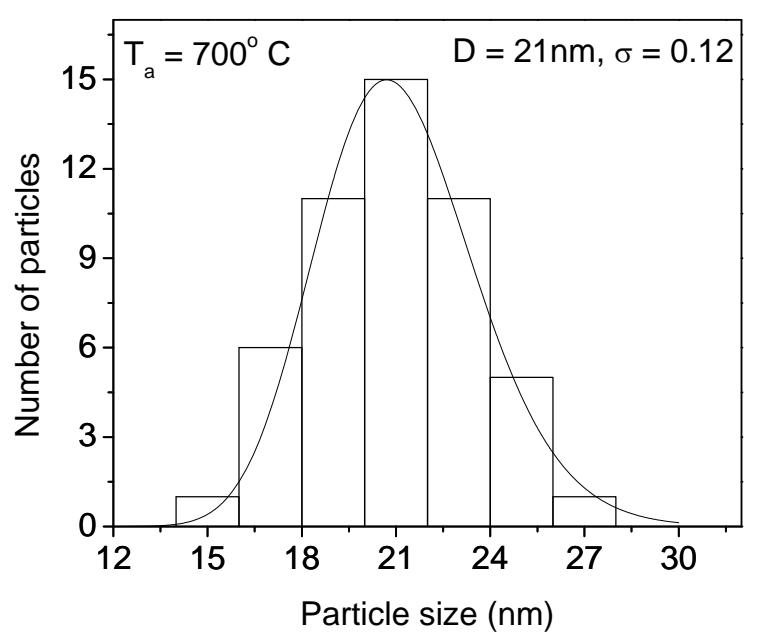

(d)

Fig. 2.11 TEM of images of the $15 \% \mathrm{Ni} / \mathrm{SiO}_{2}$ annealed at $\mathrm{T}_{\mathrm{a}}=600^{\circ} \mathrm{C}$ (a) and $\mathrm{T}_{\mathrm{a}}=700^{\circ} \mathrm{C}$ (c) respectively with the log-normal fits shown in (b) and (d) respectively. 


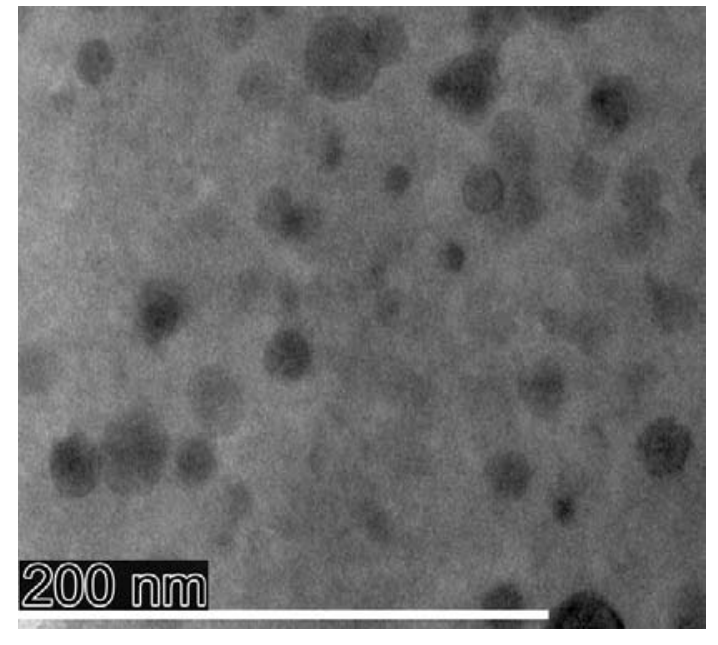

(a)

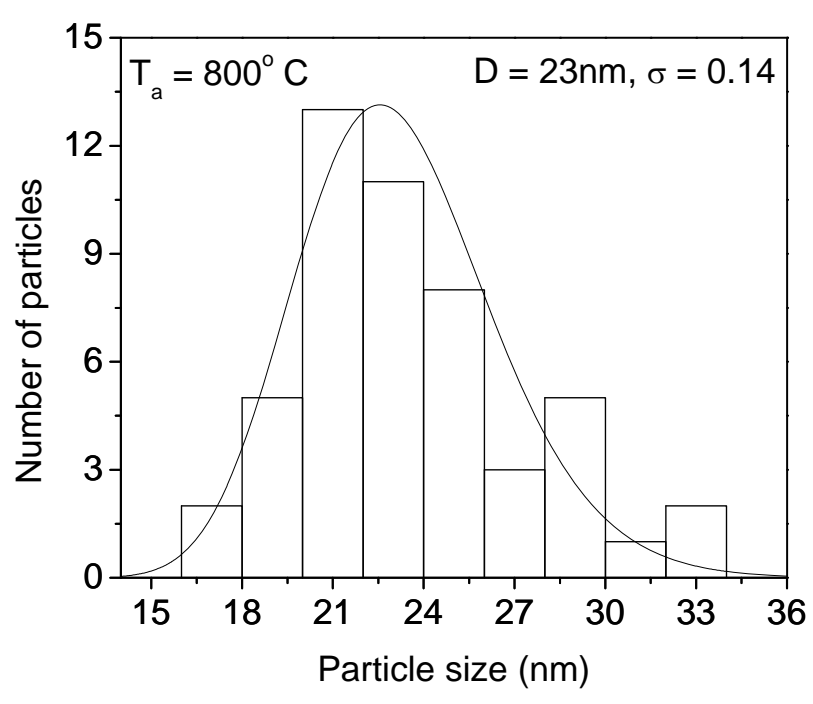

(b)

Fig. 2.12 TEM of image of the $15 \% \mathrm{Ni} / \mathrm{SiO}_{2}$ annealed at $\mathrm{T}_{\mathrm{a}}=800^{\circ} \mathrm{C}$ is shown in (a) with the log-normal fit shown in (b).

\subsection{X-ray Diffraction (XRD)}

$\mathrm{X}$-rays are produced by using high energy electrons of energy $\mathrm{eV}$ where $\mathrm{V}$ is the voltage used to accelerate the electrons and bombard a target such as $(\mathrm{Cu}, \mathrm{Fe}, \mathrm{Mo}, \mathrm{Co})$. The retardation of the electrons by the anode (target) is responsible for producing electromagnetic radiation. At high energy, the accelerated electrons knock out electrons from the inner shell of the target material thus leading the higher shell electrons to fall to the inner shell vacant position emitting radiation. X-rays are produced at the point of impact and radiate in all directions. Most of the energy of these electrons is converted into heat upon striking the target. Thus the target must be cooled continuously in order for the production of x-rays without damaging the x-ray tube. This sets a limitation on the voltage and current applied to the x-rays produced.

The outcome of the x-rays produced leads to a continuous spectra. This arises from the fact that not all the electrons are retarded in the same way. Some of the electrons 
are stopped on their first impact giving up all their energy, while others undergo a glancing impact and so they only lose their energy partially emitting x-rays. The energy E of the electron is related to the wavelength $\lambda$ of the $\mathrm{x}$-rays produced as:

$$
\begin{gathered}
E=e V=h v=\frac{h c}{\lambda} \\
\lambda=\frac{h c}{E}=\frac{6.626 \times 10^{-34} \times 3 \times 10^{8} \times 10^{10}}{1.6 \times 10^{-19} \times V}=\frac{12.424}{V(k V)} \AA
\end{gathered}
$$

Following Eq. 2.7b, electrons that lose their energy entirely emit x-rays with smaller wavelength whereas electrons which only lose their energy partially emit $\mathrm{x}$-rays with higher wavelengths. Both these processes give a continuous spectrum. If the voltage is high enough above the threshold then we observe a series of sharp lines which arise from the electrons undergoing rearrangement in the target atom (see Fig. 2.13 for $\mathrm{Cu}$ target). The continuous spectra depend on the voltage applied to the tube whereas the series of sharp intense lines depend on the nature of the target material.

It was Moseley who described the relationship of the two lines to the atomic number of the target element which came to be known as Moseley's law: $v^{1 / 2}=\mathrm{K}(\mathrm{Z}-\sigma)$ where the square root of the frequency of either of the two lines is nearly proportional to the atomic number of the target element. $\mathrm{K}$ is a universal constant for all the elements, $\mathrm{Z}$ the atomic number and $\sigma$ is another universal constant representing electron screening effect. Both $\mathrm{K}$ and $\sigma$ depend on the type of the line. The Bohr Moseley formula for $\mathrm{X}$-ray transition is given by:

$$
\begin{aligned}
& E=h v=E_{i}-E_{f}=\frac{m_{e} q_{e}{ }^{4}(Z-\sigma)^{2}}{8 h^{2} \varepsilon_{0}{ }^{2}}\left(\frac{1}{n_{i}{ }^{2}}-\frac{1}{n_{f}{ }^{2}}\right) \\
& v=\frac{m_{e} q_{e}{ }^{4}}{8 h^{3} \varepsilon_{0}{ }^{2}}\left(\frac{3}{4}\right)(Z-1)^{2}=3.29 \times 10^{15}\left(\frac{3}{4}\right)(Z-1)^{2} H z \text { for } \mathrm{k}_{\alpha} \text {-line }
\end{aligned}
$$


The wavelength of $\mathrm{CuK}_{\alpha 1}\left(\mathrm{CuK}_{\alpha 2}\right) 0.154056 \mathrm{~nm}(0.154439 \mathrm{~nm})$ with the average taken to be $0.154185 \mathrm{~nm}$ taking into consideration the differences in their intensities. Figs. 2.13 and 2.14 show the transition involved in the emission of $\mathrm{CuK}$ lines [Crystallography lab, 2007 and Kuiper, 2006].

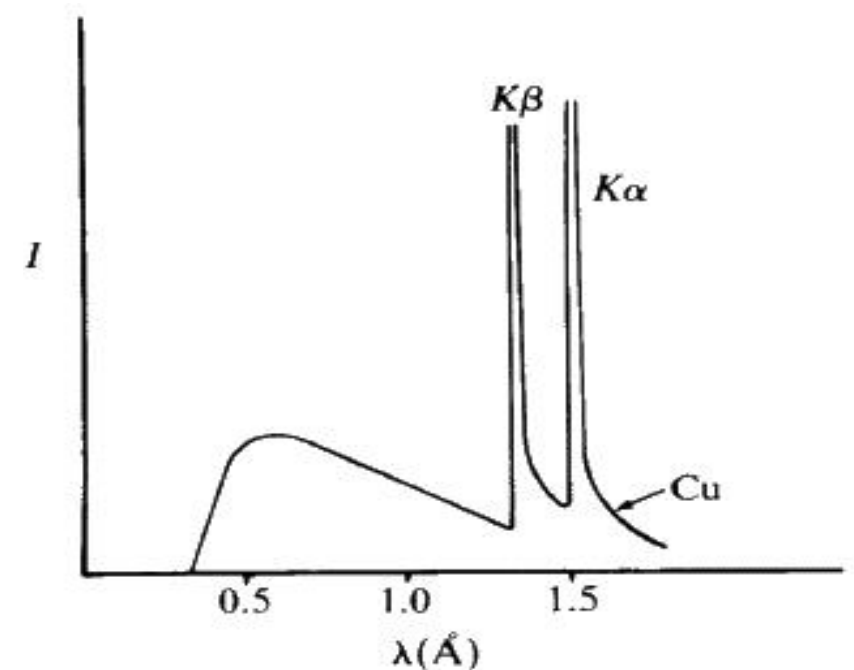

Fig. 2.13 Intensity vs. wavelength for $\mathrm{CuK}_{\alpha}$ lines.

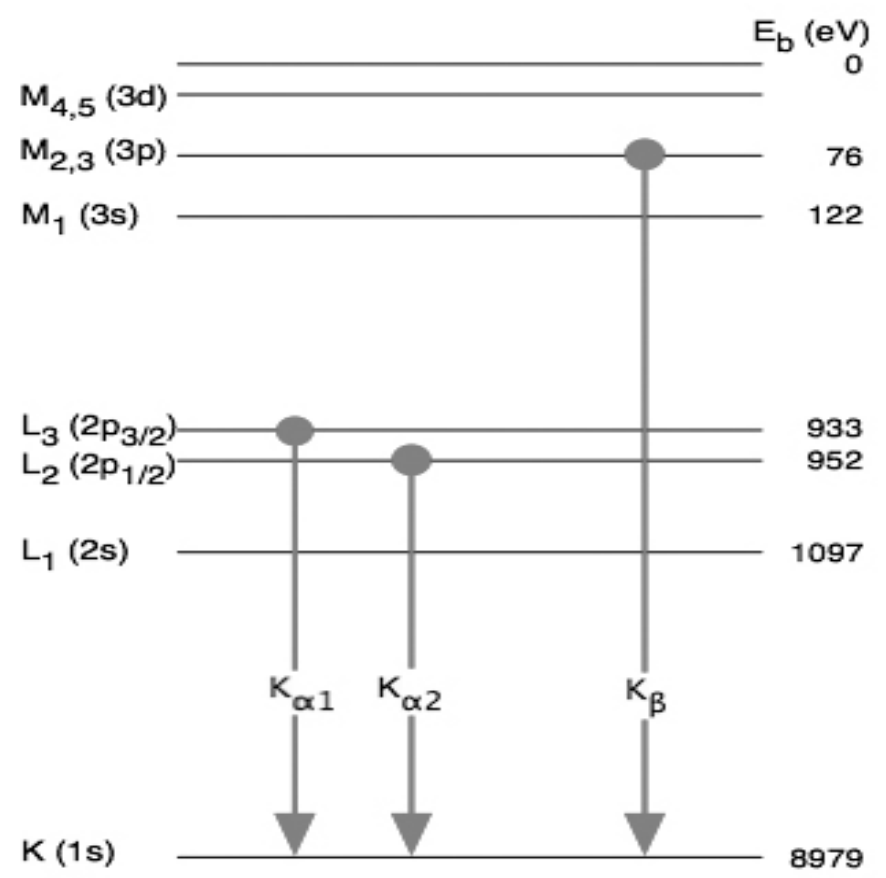

Fig. 2.14 Levels involved in CuK x-ray emission. 
The XRD pattern was obtained from Rigaku diffractometer using $\mathrm{CuK}_{\alpha}$ radiation of wavelength $0.154185 \mathrm{~nm}$. The applied voltage was $40 \mathrm{kV}$ to the target and the filament current was $30 \mathrm{~mA}$. The measurement was taken for angles $2 \theta$ varying from $5^{\circ}$ to $100^{\circ}$ in steps of $0.06^{\circ}$ in 5 second intervals. The sample holder was made of silicon and the sample was loaded on the center of the holder with a drop of ethanol/acetone in order to keep it stuck to the plate which was then placed vertically inside the x-ray diffractometer.

The analysis of the nickel nanoparticles embedded in silica matrix was done using the modified Debye Scherrer relation or the Williamson-Hall relation [Williamson et al, 1953] to obtain the particle size D:

$$
\beta \cos \theta=\frac{0.89 \lambda}{D}+\eta \sin \theta
$$

where $\beta$ is the full width at half maximum (FWHM) in radians corrected for instrumental width $b . \theta$ is the diffracting angle, $\lambda$ is the wavelength of the incident monochromatic radiation, $\eta$ is the strain present in the sample.

The particle sizes for the Ni NPs, obtained by the plots of $\beta \cos \theta$ vs. $\sin \theta$ in Fig. 2.15 , were done for the various particles sizes and the intercept at $\sin \theta=0$ determines the diameter of these particles and the slopes of the graph gave the $\eta$ values. The measured width $B$ is corrected for the instrumental width $b$ yielding $\beta^{2}=B^{2}-b^{2}$ [Ibrahim et al, 1992]. The results are summarized in Table 2.1 where the average D's measured by TEM are also included for comparison. There is a good agreement between D measured by TEM and XRD except for the largest particles where $\mathrm{D}(\mathrm{TEM})>\mathrm{D}(\mathrm{XRD})$. This difference is most likely due to the fact that TEM measures the physical size whereas in XRD, only grain size is measured. This implies that the larger particles may consist of more than one grain if Ni. It is also evident that the strain is the largest for the smallest particle with $\mathrm{D}=3.8 \mathrm{~nm}$. 

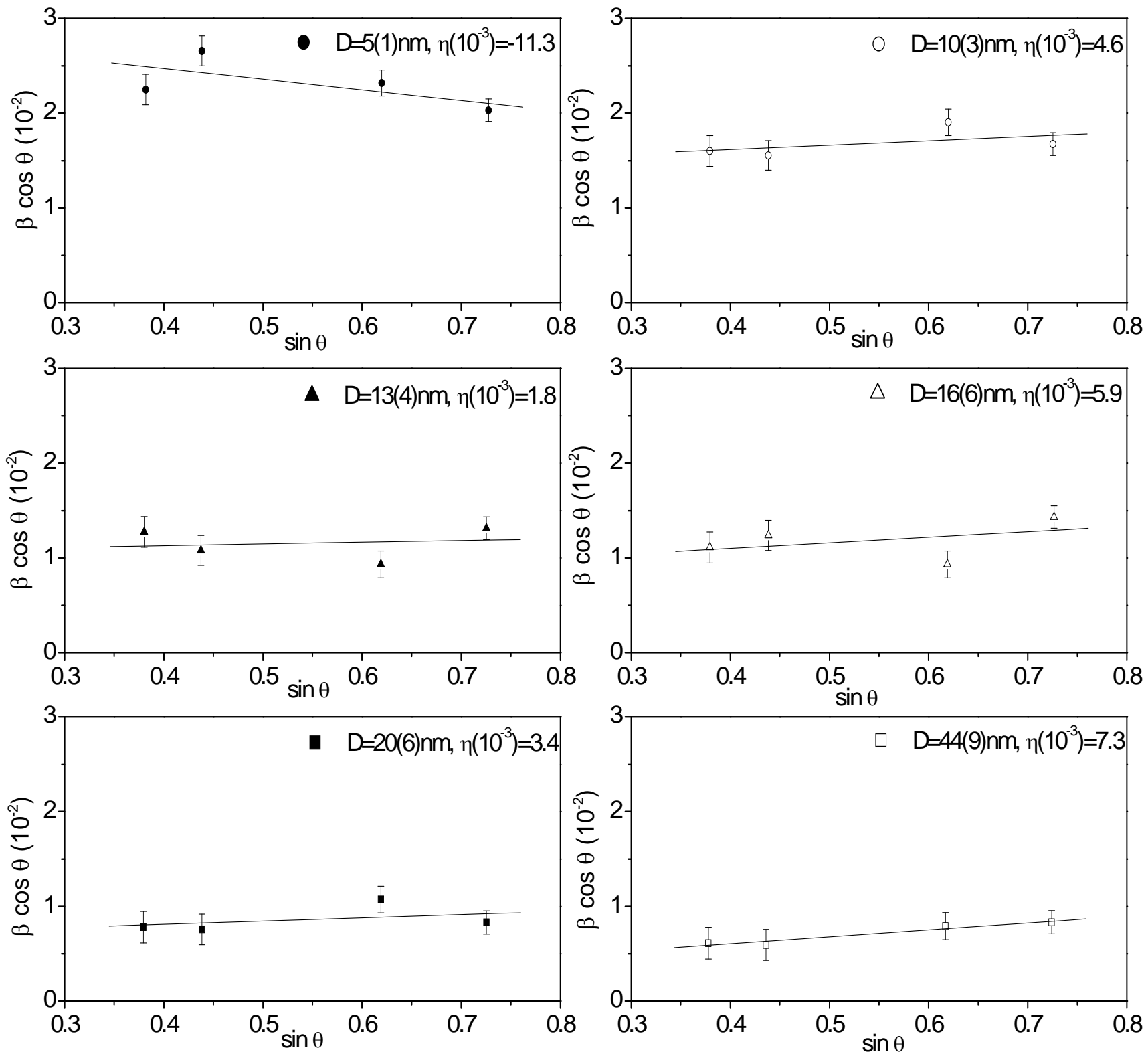

Fig. 2.15 Plots of $\beta \cos \theta$ vs. $\sin \theta$ from the Williamson-Hall relation to determine $\mathrm{D}$ and $\eta$ in Eq. 2.9. 
Table 2.1 Summary of the particle sizes obtained from XRD and TEM.

\begin{tabular}{|c|c|c|c|}
\hline $\begin{array}{c}\text { Annealing } \\
\text { temperature } \\
\mathrm{T}_{\mathrm{a}}\left({ }^{\circ} \mathrm{C}\right)\end{array}$ & $\begin{array}{c}\text { Particle } \\
\text { diameter }(\mathrm{nm}) \\
\text { TEM }\end{array}$ & $\begin{array}{c}\text { Particle } \\
\text { diameter }(\mathrm{nm}) \\
\mathrm{x} \text {-ray }\end{array}$ & $\eta\left(10^{-3}\right)$ \\
\hline 400 & $3.8(0.2)$ & $5(1)$ & -11.3 \\
\hline 500 & $11.7(0.17)$ & $10(3)$ & 4.6 \\
\hline 600 & $15(0.17)$ & $13(4)$ & 1.8 \\
\hline 700 & $21(0.12)$ & $16(6)$ & 5.9 \\
\hline 800 & $23(0.14)$ & $20(6)$ & 3.4 \\
\hline Bulk & & $44(9)$ & 7.3 \\
\hline
\end{tabular}




\subsection{Structure Factor for Nickel}

In a well grounded polycrystalline material, for the crystallites following the Bragg's law $2 \mathrm{~d}_{\mathrm{hkl}} \sin \theta=\mathrm{n} \lambda$, a diffraction line is observed. Structure factor is used to determine which (hkl) lines will be present and which will be missing. The x-ray diffraction patterns from the nanocomposite $\mathrm{Ni} / \mathrm{SiO}_{2}$ shows the presence of nickel peaks superimposed on the $\mathrm{SiO}_{2}$ background. $\mathrm{SiO}_{2}$ is amorphous in nature and hence lacks crystallinity and thus does not show multiple reflection peaks from its presence. The broad peak near $2 \theta=20^{\circ}$ in Fig. 2.5 is due to the amorphous silica. On the other hand, nanocrystals of $\mathrm{Ni}$ of sizes $>2 \mathrm{~nm}$ contain ordered crystal planes and shows multiple reflection peaks from which one can determine the structure of nickel. The structure factor that is responsible for the presence and intensity of the allowed Bragg lines is given by [e.g. see Kittel, 1996]:

$$
S(h k l)=\sum_{j} f_{j} \exp \left[-i 2 \pi\left(h x_{j}+k y_{j}+l z_{j}\right)\right]
$$

Here $f_{j}$ is the atomic scattering factor of the $j^{\text {th }}$ atom in the unit cell with coordinates $x_{j}, y_{j}$ and $z_{j}$ and it involves the number and distribution of atomic electrons, the wavelength and the angle of scattering of the radiation. Nickel has an fcc lattice and has identical atoms located at $(000),(0,1 / 2,1 / 2),(1 / 2,0,1 / 2)$ and $(1 / 2,1 / 2,0)$ giving the structure factor:

$$
S(h k l)=f(\theta)[1+\exp [-i \pi(k+l)]+\exp [-i \pi(h+l)]+\exp [-i \pi(h+k)]]
$$

The form factor $\mathrm{f}$ is the same here since all the nickel atoms are equivalent. In Eq. 2.11, if all the indices are even or odd, the structure factor is $4 \mathrm{f}$, and if they consist of even and odd numbers, then $\mathrm{S}=0$ and hence no reflection is observed. The allowed (hkl) lines for Ni for which S is non-zero are (111), (200), (220), (311) and (222). The overall intensity of the diffracted beam for a diffractometer is given by [Klug et al, 1954]: 


$$
I=\left(\frac{I_{o} e^{4}}{m^{2} c^{4}}\right)\left(\frac{\lambda^{3} A}{32 \pi r}\right)\left(\frac{1}{V^{2}}\right)\left[|S|^{2} p\left(\frac{1+\cos ^{2} 2 \theta}{\sin ^{2} \theta \cos \theta}\right) \frac{e^{-\frac{2 B \sin ^{2} \theta}{\lambda^{2}}}}{2 \mu}\right]
$$

Here I is integrated intensity, $I_{0}$ is intensity of incident beam, e, $m$ are charge and mass of the electron, $\mathrm{c}$ is velocity of light, $\lambda$ is wavelength of incident radiation, $\mathrm{r}$ is the radius of the diffractometer circle, $\mathrm{A}$ is cross-sectional area of the incident beam, $\mathrm{V}$ is the volume of the unit cell, $\mathrm{S}$ is structure factor, $\mathrm{p}$ is the multiplicity factor of the (hkl) line, $\theta$ is Bragg angle, $\mathrm{B}$ is Debye-Waller factor, $\mu$ is the linear absorption factor of the specimen.

For a given sample and a diffractometer, the intensity of an allowed diffracted line depends on $|S|^{2}$, $p$ and the Lorentz polarization (LP) factor $\left(1+\cos ^{2} 2 \theta\right) /\left(\sin ^{2} \theta \cos \theta\right)$. For the Bragg lines of Ni, p=8, 6, 12, 24 and 6 for the (111), (200), (220), (311) and (400) lines respectively. Both the atomic scattering factor $\mathrm{f}$ and the LP factor decrease as $\theta$ increases. So the intensity of a given line depends on several factors and it is more difficult to analyze than the position and width of the lines. The Debye-Waller also changes with $\theta$ as shown and $\mathrm{B}$ increases with increase in temperature making the intensity of a line decrease with increase in temperature due to increasing amplitude of atomic oscillations. All of these factors must be taken into account to accurately determine B. 


\section{CHAPTER III}

\section{Superparamagnetism: Theoretical Considerations}

In order to make the discussion of the results presented in chapter 5 later easier to follow, here a brief introduction to the relevant theoretical concepts are outlined using readily available information from literature [e.g. see Cullity, 1972]. With the absence of any interaction among the individual magnetic moments in a material, one can obtain the Curie law variation of magnetization. Along with the development of the molecular field theory based on quantum mechanics the concept of exchange interaction was introduced between two electrons at two sites which is now believed to be responsible for magnetism. Further the band theory of magnetism used was able to explain the presence of magnetism in metals such as $\mathrm{Ni}$. Crystalline anisotropy was introduced from the fact that the magnetization lies along certain crystallographic axis. Finally, the formation of single domain particles below a critical size which leads to superparamagnetism emerges.

\subsection{Introduction}

The first model to explain ferromagnetism was the hypothesis of the molecular field by Pierre Weiss in 1906, which further leads to the Curie Weiss law. Above the Curie ordering temperatures, magnetic ordering is destroyed by thermal fluctuations and the system becomes paramagnet and follows the Curie Weiss law. Some substances were found spontaneously magnetized in the absence of an applied field, however they could also be found in a demagnetized state. Weiss was able to answer this question with the explanation through domains. The two essential postulates of Weiss were spontaneous magnetization and division into domains [Cullity, 1972].

In a ferromagnetic material, there must be a strong interaction present that tends to align the atomic dipoles parallel. This interaction can be considered to be equivalent to some internal field $\mathrm{H}_{\mathrm{m}}$. The Curie temperature $\mathrm{T}_{\mathrm{C}}=627 \mathrm{~K}$ for Ni gives the magnitude of this internal field using the approximate relation:

$$
\mu_{B} H_{m} \approx k_{B} T_{C}
$$


yielding for $\mathrm{H}_{\mathrm{m}} \approx 10^{7} \mathrm{Oe}$ for Ni. The Weiss model did not provide any explanation for the origin for such a large internal field. However it was assumed that $\mathrm{H}_{\mathrm{m}}$ is proportional to the spontaneous magnetization given by:

$$
H_{m}=N_{W} M
$$

With the presence of the internal field the total field acting on the sample is given by:

$$
H_{\text {effective }}=H_{\text {applied }}+N_{w} M
$$

\subsection{Curie-Weiss Law}

If the internal field or the molecular field due to inter-dipole interaction is nonzero then the Curie law $(\mathrm{M}=\mathrm{CH} / \mathrm{T})$ changes to:

$$
M=\frac{C\left(H+N_{W} M\right)}{T}
$$

leading to $\chi=\frac{M}{H}=\frac{C}{T-\theta}$, with $\theta=C N_{W}$

According to Eq. 3.4, $\chi \rightarrow \infty$ when $\mathrm{T} \rightarrow \theta$, yielding the ferromagnetic ordering temperature $\mathrm{T}_{\mathrm{C}}=\theta=\mathrm{CN}_{\mathrm{W}}$. When $\mathrm{N}_{\mathrm{W}}=0, \theta=0$ leading to the Curie Law $\chi=\mathrm{C} / \mathrm{T}$.

\subsection{Magnetization of Non-interacting Magnetic Dipoles}

Suppose there are $\mathrm{N}$ paramagnetic atoms per unit volume of a sample each having magnetic moments distributed randomly leading to zero net moment. With the application of an external magnetic field $\mathrm{H}$, there will be some alignment of the magnetic moments along the field. The magnetization of that specimen is then given by:

$$
M=N g \mu_{B} J B_{J}(y)
$$

where $\mu_{\mathrm{B}}$ is the bohr magneton, $\mathrm{g}$ is Landé factor, $\mathrm{J}$ is the angular momentum quantum number, $y=\frac{J g \mu_{B} H}{k T}$ and $\mathrm{B}_{\mathrm{J}}(\mathrm{y})$ is the Brillouin function given by [Morrish, 2001]:

$$
B_{J}(y)=\frac{2 J+1}{2 J} \operatorname{coth}\left(\frac{2 J+1}{2 J}\right) y-\frac{1}{2 J} \operatorname{coth}\left(\frac{y}{2 J}\right)
$$

The condition when $\mathrm{J} \rightarrow \infty$ (classical case), $\mathrm{B}_{\mathrm{J}}(\mathrm{y})$ becomes the Langevin function: 


$$
\underset{J \rightarrow \infty}{B_{J}(y)}=\operatorname{coth}(y)-\frac{1}{y}=L(y)
$$

At sufficiently large fields, all the dipoles will be aligned along the field yielding the saturation magnetization $\mathrm{M}_{\mathrm{S}}$ :

$$
M_{S}=N g \mu_{B} J
$$

\subsection{Curie Law}

When the Zeeman splitting is much smaller than the thermal energy, that is $\mathrm{y}<<1$, $B_{J}(y) \approx \frac{1}{3} \frac{J+1}{J} y$ and Eq. 3.5 reduces to:

$$
M=\frac{N g^{2} J(J+1) \mu_{B}{ }^{2} H}{3 k T}
$$

This yields the Curie law variation of the magnetization for non-interacting dipoles viz.

$$
\chi=\frac{M}{H}=\frac{C}{T} \text { with } C=\frac{N g^{2} \mu_{B}{ }^{2} J(J+1)}{3 k} .
$$

\subsection{Origin of Molecular Field}

With the development of quantum mechanics, it was Heisenberg who suggested the origin of the molecular field to be due to exchange interaction. For a particular pair of atoms, electrostatic attractive forces (electron-proton) and repulsive forces (electronelectron) exist, but there is an exchange interaction. Consider two identical electrons a and $\mathrm{b}$ located on two neighboring sites 1 and 2. Quantum mechanics requires that total wavefunction of two electron system must be antisymmetric with respect to interchange of the electrons. The symmetric and anti-symmetric wavefunction of the two electron system are:

$$
\begin{aligned}
& \psi_{S}=\frac{1}{\sqrt{2}}\left\{\psi_{a}(1) \psi_{b}(2)+\psi_{b}(1) \psi_{a}(2)\right\} \\
& \psi_{A}=\frac{1}{\sqrt{2}}\left\{\psi_{a}(1) \psi_{b}(2)-\psi_{b}(1) \psi_{a}(2)\right\}
\end{aligned}
$$

The electrostatic coulomb interaction energy for the two electron system is then given by: 


$$
\begin{gathered}
W=\frac{1}{2} \int\left[\psi_{a}^{*}(1) \psi_{b}^{*}(2) \pm \psi_{b}{ }^{*}(1) \psi_{a}^{*}(2)\right] \frac{e^{2}}{r_{12}}\left[\psi_{a}(1) \psi_{b}(2) \pm \psi_{b}(1) \psi_{a}(2)\right] d^{3} r_{1} d^{3} r_{2} \\
W=\frac{1}{2} \int\left|\psi_{a}(1)\right|^{2} \frac{e^{2}}{r_{12}}\left|\psi_{a}(2)\right|^{2} d^{3} r_{1} d^{3} r_{2}+\int\left|\psi_{b}(1)\right|^{2} \frac{e^{2}}{r_{12}}\left|\psi_{a}(2)\right|^{2} d^{3} r_{1} d^{3} r_{2} \\
\pm \int \psi_{a}^{*}(1) \psi_{b}{ }^{*}(2)\left(\frac{e^{2}}{r_{12}}\right) \psi_{b}(1) \psi_{a}(2) d^{3} r_{1} d^{3} r_{2} \pm \int \psi_{b}^{*}(1) \psi_{a}^{*}(2)\left(\frac{e^{2}}{r_{12}}\right) \psi_{a}(1) \psi_{b}(2) d^{3} r_{1} d^{3} r_{2} \\
W=K \pm J_{e x} \\
K=\int\left|\psi_{a}(1)\right|^{2}\left(\frac{e^{2}}{r_{12}}\right)\left|\psi_{b}(2)\right|^{2} d^{3} r_{1} d^{3} r_{2} \\
J_{e x}=\int \psi_{a}^{*}(1) \psi_{b}^{*}(2)\left(\frac{e^{2}}{r_{12}}\right) \psi_{b}(1) \psi_{a}(2) d^{3} r_{1} d^{3} r_{2}
\end{gathered}
$$

where $\mathrm{K}$ is known as the Coulomb term and $\mathrm{J}_{\mathrm{ex}}$ is known as the exchange term. $\mathrm{J}_{\mathrm{ex}}$ is called the exchange energy since the integral involves the interchange of electrons a and $\mathrm{b}$ between site 1 and 2 . This term only because of the symmetry requirement imposed by quantum mechanics and Pauli's exclusion principle.

We write the Hamiltonian for the system of two electrons as:

$$
\begin{gathered}
H_{a b}=K_{a b}-\frac{1}{2} J_{e x}\left(1+4 \vec{S}_{a} \cdot \vec{S}_{b}\right) \\
\text { and }\left\langle 2 \vec{S}_{a} \cdot \vec{S}_{b}\right\rangle=\left[S(S+1)-S_{a}\left(S_{a}+1\right)-S_{b}\left(S_{b}+1\right)\right]
\end{gathered}
$$

For the triplet state total $\mathrm{S}=1$, for $\mathrm{S}_{\mathrm{a}}=\mathrm{S}_{\mathrm{b}}=1 / 2$ and $\left\langle 2 \vec{S}_{a} \cdot \vec{S}_{b}\right\rangle=\left[2-\frac{3}{4}-\frac{3}{4}\right]=\frac{1}{2}$,

$$
\left\langle H_{a b}\right\rangle=K_{a b}-\frac{1}{2} J_{e x}(1+1)=K_{a b}-J_{e x}
$$

and for the singlet state $S=0$, for $S_{a}=S_{b}=1 / 2$ and $\left\langle 2 \vec{S}_{a} \cdot \vec{S}_{b}\right\rangle=\left[0-\frac{3}{4}-\frac{3}{4}\right]=-\frac{3}{2}$

$$
\left\langle H_{a b}\right\rangle=K-\frac{1}{2} J_{e x}(1-3)=K+J_{e x} .
$$

So the Hamiltonian Eq. 3.15 gives the energy of the triplet state being lower than that of the singlet state by $2 \mathrm{~J}_{\mathrm{ex}}$, for positive $\mathrm{J}_{\mathrm{ex}}$. If $\mathrm{J}_{\mathrm{ex}}<0$, then the singlet state with $\mathrm{S}=0$ is the 
lower energy state yielding anti-ferromagnetism. The Hamiltonian with spin-dependent and spin-independent term is then given by:

$$
H_{a b}=K-\frac{1}{2} J_{e x}-2 J_{e x} \vec{S}_{a} \cdot \vec{S}_{b}
$$

The first two terms of Eq. 3.16 are independent of spin so they will be the same for triplet and singlet. We can get the spin dependent exchange term from the last part of Eq. 3.16 given by:

$$
H_{a b}=-2 J_{e x} S_{i} \cdot S_{j}=-2 J_{e x} S_{i} S_{j} \cos \phi
$$

where $\phi$ is the angle between the spins.

\subsection{Relation between Molecular Field and Exchange Constant}

If there are $\mathrm{Z}$ nearest neighbors then we get the exchange interaction as:

$$
\begin{gathered}
H_{a b}=-2 \sum_{a \neq b}^{n n} J_{e x} \vec{S}_{a} \cdot \vec{S}_{b} \\
\left\langle H_{a b}\right\rangle=-2 J_{e x}\left\langle\vec{S}_{a}\right\rangle \cdot \sum_{b=1}^{Z}\left\langle\vec{S}_{b}\right\rangle
\end{gathered}
$$

Using the relation $\mathrm{H}_{\mathrm{m}}=\mathrm{N}_{\mathrm{W}} \mathrm{M}$ and $\mathrm{M}=\mathrm{Ng} \mu_{\mathrm{B}} \mathrm{S}$ we obtain:

$$
\begin{aligned}
\left\langle H_{a b}\right\rangle=-2 J_{e x}\left\langle\vec{S}_{a}\right\rangle \cdot \sum_{b=1}^{Z}\left\langle\vec{S}_{b}\right\rangle & =-M \cdot H_{m}=-g \mu_{B}\left\langle\vec{S}_{a}\right\rangle H_{m} \\
g \mu_{B} H_{m} & =2 J_{e x} Z\left\langle S_{b}\right\rangle \\
H_{m}=N_{W} M=\frac{2 J_{e x} Z}{g \mu_{B}}\left\langle S_{b}\right\rangle & =N_{W} N g \mu_{B}\left\langle S_{b}\right\rangle \\
N_{W} & =\frac{2 J_{e x} Z}{N g^{2} \mu_{B}^{2}} \\
\text { and } N_{W} & \propto J_{e x}
\end{aligned}
$$

Using $\mathrm{T}_{\mathrm{C}}=\theta=\mathrm{CN}_{\mathrm{W}}$ leads to:

$$
T_{C}=\frac{2 J_{e x} Z J(J+1)}{3 k}
$$


According to Eq. 3.21, $\mathrm{T}_{\mathrm{C}}$ is proportional to $\mathrm{J}_{\mathrm{ex}}$, the exchange constant between the nearest neighbors. Thus magnetic ordering is related to the strength of exchange coupling among the magnetic moments.

\subsection{Band Theory of Nickel}

An isolated nickel atom has an electronic configuration of $3 \mathrm{~d}^{8} 4 \mathrm{~s}^{2}$. According to band theory, the allowed energy states occur in bands of energy often separated by energy gap. In $\mathrm{Ni}, 3 \mathrm{~d}$ and $4 \mathrm{~s}$ bands overlap. The energy bands of $\mathrm{Ni}$ only fill 9.46 electrons per atom up to the fermi level for the $3 \mathrm{~d}$ band and 0.54 electrons per atom of the 4s band [Kittel, 1996].

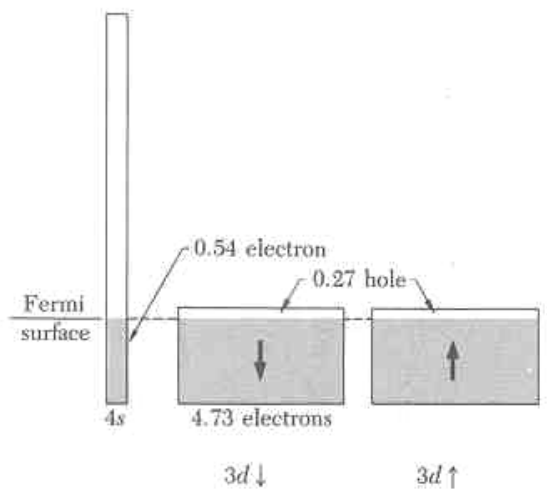

(a)

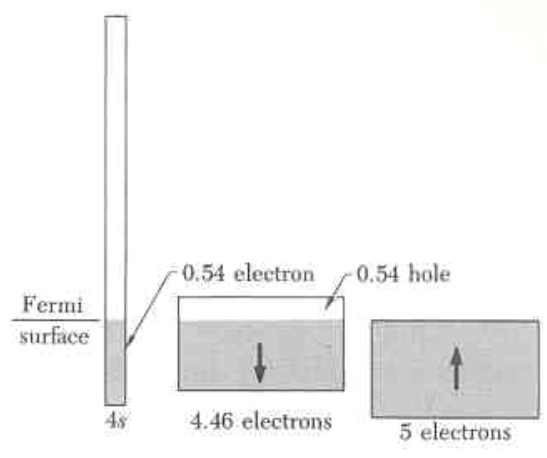

$3 d \downarrow$

(b)

Fig. 3.1 Band relationship of $\mathrm{Ni}$ above and below the Curie temperature.

Fig. 3.1a shows that the $3 \mathrm{~d} \uparrow$ and the $3 \mathrm{~d} \downarrow$ levels are filled equally for Ni above the Curie temperature and hence the net magnetic moment is zero. Fig. 3.1b shows that for below the Curie temperature, ferromagnetic ordering takes place because of exchange coupling which causes a shift between the spin-up and spin-down states. This causes a net magnetic moment due to the excess population of electrons from one band over the other, yielding a magnetic moment $\mu=0.54 \mu_{\mathrm{B}} /$ atom. 


\subsection{Anisotropy in Bulk Nickel}

The origin of crystalline anisotropy arises from the fact that crystals are inherently anisotropic and that the magnetization lies along a certain crystallographic axis. Fig. 3.2 below shows the M vs. H plots for $\mathrm{H}$ applied along the [111], [110] and [100] directions in Ni. From these measurements it can be seen that for $\mathrm{H} \|$ [111] requires smaller field to saturate the magnetization than $\mathrm{H}$ parallel to [100] or [110] directions. Thus the moments are aligned along the [111] direction. If the sample shape lacks spherical symmetry, then another source is shape anisotropy hence the presence of demagnetization energy. Also due to the presence of spins on the surface the surface anisotropy plays an important role as the spins on the surface experience a different force than the spins within the material. For a bulk sample the surface anisotropy can be considered to be negligible as most of the spins are inside the material.

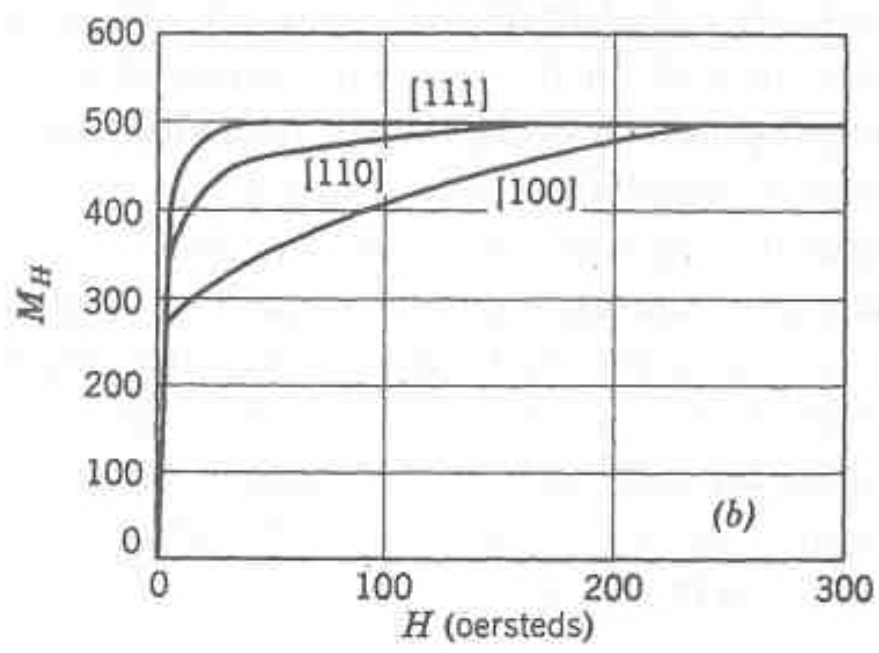

Fig. 3.2 Magnetization curve for single crystal Ni [Kittel, 1996]. 


\subsection{Single Domain Particles}

Consider the case that when there is no applied field and the magnetization of the particle is stable and it does not change with time. Let us consider a single domain ellipsoidal particle which simulates a uniaxial anisotropy due to shape from the demagnetization factors $\mathrm{D}_{\mathrm{a}}$ and $\mathrm{D}_{\mathrm{b}}$. The E/volume of such a particle is given by the Eq. 3.22a below [Morrish, 2001].

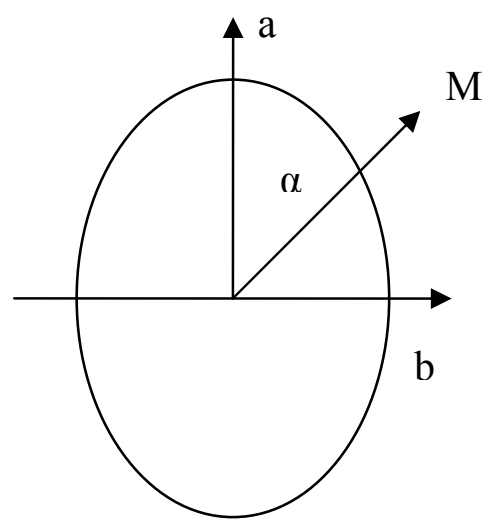

Fig. 3.3 Ellipsoidal particle with a and $\mathrm{b}$ as major and minor axis.

$$
\begin{aligned}
E & =\frac{1}{2}\left((M \cos \alpha)^{2} D_{a}+(M \sin \alpha)^{2} D_{b}\right) \\
\text { or } E & =\frac{1}{2} M^{2} D_{a}+\frac{1}{2} M^{2}\left(D_{b}-D_{a}\right) \sin ^{2} \alpha
\end{aligned}
$$

Here $D_{a}$ is the longitudinal demagnetization factor and $D_{b}$ is the transverse demagnetization factor. For a spherical particle $\mathrm{D}_{\mathrm{a}}=\mathrm{D}_{\mathrm{b}}$. This angle dependent energy for a particle with volume $\mathrm{V}$ is then:

$$
E=\frac{1}{2} C V \sin ^{2} \alpha \text { where } C=M^{2}\left(D_{b}-D_{a}\right)
$$

where $\alpha$ is the angle between the magnetization and the easy direction and $\mathrm{C}$ is the anisotropy constant. The direction for minimum energy is obtained for $\alpha=0$ or $\alpha=\pi$, where the barrier between these minimum energy directions is $\mathrm{CV} / 2(\alpha=\pi / 2)$. For the 
magnetization to switch directions the system must be perturbed. One way the system will be perturbed is by the presence of thermal energy. The other is that the energy barrier is lowered if the volume of the particle is small. Particles whose magnetization changes spontaneously are said to be paramagnetic but if their moments are large than they are called superparamagnetic particles.

In order to characterize the process with some relaxation time, we consider the fact that the particles easy axis lies along the z-axis and we apply an external field along the z-axis so the magnetization is aligned along the z-axis. Upon removal of the field the magnetization will decay as a function of time according to:

$$
M=M_{o} e^{-\frac{t}{\tau}}
$$

where $1 / \tau$ is the sum of the probability per second for a single transition from $+\mathrm{z}$ to $-\mathrm{Z}$ and from $-z$ to $+z$. The frequency of measurement given by $f=1 / \tau$ and is proportional to the Boltzmann factor $e^{-\frac{C V}{2 k T}}$ leading to:

$$
f=f_{o} e^{-\frac{E a}{k_{B} T}}
$$

where $\mathrm{E}_{\mathrm{a}}=1 / 2 \mathrm{CV}$ is the anisotropy barrier and $\mathrm{f}_{\mathrm{o}}$ is the attempt frequency $\simeq 10^{10} \mathrm{~Hz}$, equal to the frequency of precession in a magnetic field $\left(h v=g \mu_{B} H\right)$. Eq. 3.25 is called the Néel-Arrhenius relaxation of the magnetization of a single domain particle. It is evident from Eq. 3.25 that as temperature $\mathrm{T}$ decreases, the switching frequency $\mathrm{f}$ decreases that is it takes longer for the moments to relax. When $f$ becomes equal to $f_{m}$, the frequency of measurement, then the moment is blocked from switching that is the moments are frozen. This temperature, called the blocking temperature $\left(\mathrm{T}_{\mathrm{B}}\right)$ then follows from Eq. 3.25 as:

$$
T_{B}=\frac{E_{a} / k}{\ln \frac{f_{o}}{f_{m}}}
$$

where $E_{a}=1 / 2 C V=K V, K$ being the uniaxial anisotropy. According to Eq. 3.26, $T_{B}$ increases if the measuring frequency $f_{m}$ is increased. This equation is tested experimentally in chapter 5 . 


\subsection{Variation in Blocking Temperature}

\section{A. Blocking temperature and particle size}

From Eq. 3.26 we see that for a unique volume $\mathrm{V}$ there is a unique blocking temperature $T_{B}$. Since the blocking temperature is proportional to the volume of the particle, there is a distribution of blocking temperatures if there is a distribution of particle sizes. If we have a narrow distribution in particle sizes then we can get the average blocking temperature from the peak in the $\mathrm{M}$ vs. $\mathrm{T}$ curve for a given applied field. Above the temperature at which the bifurcation of the zero field cooled curve and the field cooled curve takes place, all the particles are unblocked and the system is in the superparamagnetic state.

B. Variation of $T_{B}$ with applied field

Theoretically it has been known that the energy barrier is lowered by the applied field. So the blocking temperature is also dependent on the applied field and it is known to shift to lower temperatures with the increase in the applied dc field. As the applied field increases the energy barrier decreases so that the particles get unblocked at lower temperatures.

The energy barrier $\Delta \mathrm{E}$ follows the relation given by [Walton, 1990 and Victora, 1989]:

$$
\Delta E=K V\left[1-\frac{H}{H_{0}}\right]^{m}
$$

where $\mathrm{H}_{\mathrm{o}}$ is the field where $\Delta \mathrm{E} \rightarrow 0$.

Eq. 3.27 can be written in terms of the blocking temperature as:

$$
T_{B}(H)=T_{B 0}\left[1-\frac{H}{H_{0}}\right]^{m}
$$

where $\mathrm{m}=3 / 2$ to 2 have been predicted [Walton, 1990 and Victora, 1989]. We provide a test for this equation in chapter 5 . 
C. Langevin function and magnetic moment per particle

The particles whose magnetization is subject to change due to thermal agitation are similar to paramagnetic atoms, except that their moment is very large. This phenomenon is called superparamagnetism which was predicted by Néel [Morrish, 2001]. In order to see whether the particles are superparamagnetic, $\mathrm{M}$ vs. $\mathrm{H}$ measurements are done at various temperatures above $T_{B}$ and then fit to the langevin function given by:

$$
\begin{gathered}
\mathcal{L}(x)=\operatorname{coth}(x)-\frac{1}{x} \\
M=M_{o} \mathcal{L}\left(\frac{\mu_{p} H}{k_{B} T}\right)+\chi_{a} H
\end{gathered}
$$

were $M_{o}$ is the saturation magnetization, $\chi_{\mathrm{a}}$ is the high field susceptibility and $\mu_{\mathrm{P}}$ is the effective magnetic moment/particle.

D. Magnetic hysteresis and coercivity $\left(\mathrm{H}_{\mathrm{C}}\right)$

For $\mathrm{T}<\mathrm{T}_{\mathrm{B}}$, the magnetic moments are frozen in space and time and hence a nanoparticles system behaves like a ferromagnet. For the hysteresis loop measurements of the plots of $\mathrm{M}$ vs. $\mathrm{H}$ for $\mathrm{T}<\mathrm{T}_{\mathrm{B}}$ the coercivity $\mathrm{H}_{\mathrm{C}}$ can be determined. Theoretically, it has been shown that $\mathrm{H}_{\mathrm{C}}$ should vary with temperature as [Fonseca, 2002]:

$$
H_{C}(T)=H_{C O}\left[1-\left(\frac{T}{T_{B}}\right)^{\frac{1}{2}}\right]
$$

so that $\mathrm{H}_{C} \rightarrow 0$ as $\mathrm{T} \rightarrow \mathrm{T}_{\mathrm{B}}$. This equation is tested by experimental measurements in Ni NPs in chapter 5 . 


\section{CHAPTER IV}

\section{Background Theory on Magnetic Relaxation: Theoretical Considerations}

In this work, I have used electron magnetic resonance (EMR) as well as the non-resonant technique of the variation of the AC susceptibilities with change in frequency in the absence of a DC field. In this chapter basic theory of EMR using Bloch equations is presented followed by basic equations for $\mathrm{AC}$ susceptibilities.

\subsection{Bloch Equations}

The basic understanding of the phenomena of electron magnetic resonance (EMR) and nuclear magnetic resonance (NMR) can be obtained through the use and applications of the Bloch equations for magnetic resonance. In EMR, the total magnetic moment of an ion is $\mu=-\gamma \mathrm{J}$ where $\mathrm{J}$ is the total angular momentum and $\gamma=\mathrm{g} \mu_{\mathrm{B}} / \mathrm{h}$. In magnetic field $H$, this dipole moment experiences a torque $\vec{\mu} \times \vec{H}$ leading to:

$$
\frac{d \vec{\mu}}{d t}=-\gamma \vec{\mu} \times \vec{H} .
$$

Since the total magnetization $\mathrm{M}=\mathrm{N} \mu, \mathrm{N}$ being the total number of moments per unit volume, one gets the equation of motion:

$$
\frac{d \vec{M}}{d t}=-\gamma \vec{M} \times \vec{H}
$$

In magnetic resonance:

$$
H=H_{o} \hat{k}+H_{1}(\cos (\omega t) \hat{i}+\sin (\omega t) \hat{j})
$$

where the rf field of frequency $\omega$ applied in the xy plane is usually much smaller than the static field $\mathrm{H}_{\mathrm{o}}$ applied along the $\mathrm{z}$ direction i.e. $\mathrm{H}_{1}<<\mathrm{H}_{\mathrm{o}}$. Substituting for $\mathrm{H}$ in Eq. 4.1 and assuming $\mathrm{M}_{\mathrm{x}}, \mathrm{M}_{\mathrm{y}}$ and $\mathrm{M}_{\mathrm{Z}}$ lead to the resonance condition:

$$
\omega_{o}=\gamma H_{o}
$$

Eq. 4.3 is often used to determine $\gamma$ and hence the g-value of the ion. For free electrons $\mathrm{g}=2.0023$. 
The Bloch equations are obtained from Eq. 4.1 by including the damping terms leading to [Morrish, 2001]:

$$
\begin{aligned}
\frac{d M_{x}}{d t} & =-\gamma(M \times H)_{x}-\frac{M_{x}}{T_{2}} \\
\frac{d M_{y}}{d t} & =-\gamma(M \times H)_{y}-\frac{M_{y}}{T_{2}} \\
\frac{d M_{z}}{d t} & =-\gamma(M \times H)_{z}-\frac{M_{z}-M_{o}}{T_{1}}
\end{aligned}
$$

By substituting for $H$ in Eqs. 4.4, one needs to solve for $M_{x}, M_{y}$, and $M_{z}$ under the steady state condition $\mathrm{dM}_{\mathrm{z}} / \mathrm{dt}=0$. The solution is facilitated by defining $\mathrm{M}^{ \pm}=\mathrm{M}_{\mathrm{x}} \pm \mathrm{M}_{\mathrm{y}}$ and $\mathrm{M}^{ \pm}=$ $\mathrm{N}^{ \pm} \mathrm{e}^{ \pm \mathrm{i} \omega \mathrm{t}}$, leading to:

$$
\begin{aligned}
M_{x} & =\frac{\gamma M_{Z} H_{1} T_{2}}{1+\left(\omega_{o}-\omega\right)^{2} T_{2}^{2}}\left(T_{2}\left(\omega_{o}-\omega\right) \cos (\omega t)+\sin (\omega t)\right) \\
M_{z} & =M_{o} \frac{1+\left(\omega_{o}-\omega\right)^{2} T_{2}^{2}}{1+\left(\omega_{o}-\omega\right)^{2} T_{2}^{2}+\gamma^{2} H_{1}^{2} T_{1} T_{2}}
\end{aligned}
$$

Eq. 4.6 shows that $\mathrm{M}_{\mathrm{z}} \neq \mathrm{M}_{\mathrm{o}}=\chi_{\mathrm{o}} \mathrm{H}_{\mathrm{o}}$. Here $\mathrm{M}_{\mathrm{z}}$ is the magnetization along the $\mathrm{z}$-direction, $\mathrm{M}_{\mathrm{o}}$ is the saturation magnetization and $\chi_{\mathrm{o}}$ is the static magnetic susceptibility. Note that $M_{x}$ is time dependent and it is generated by the rf field $\mathrm{H}_{1} \mathrm{e}^{\mathrm{i} \omega \mathrm{t}}$. Therefore:

$$
\begin{aligned}
M_{x} & =\operatorname{Re} \chi \cdot H_{1} e^{i \omega t} \\
& =\operatorname{Re} H_{1}\left(\chi^{\prime}-i \chi^{\prime \prime}\right)(\cos (\omega t)+\sin (\omega t)) \\
& =\chi^{\prime} H_{1} \cos (\omega t)+\chi^{\prime \prime} H_{1} \sin (\omega t)
\end{aligned}
$$

Comparing Eqs. 4.5 and 4.7 and substituting for $\mathrm{M}_{\mathrm{z}}$ leads to the final equations for the real (dispersion) and imaginary (absorption) parts of the AC susceptibility $\chi^{\prime}$ and $\chi^{\prime \prime}$ :

$$
\begin{aligned}
& \chi^{\prime}=\frac{\omega_{o} \chi_{o} T_{2}^{2}\left(\omega_{o}-\omega\right)}{1+T_{2}^{2}\left(\omega_{o}-\omega\right)^{2}+\gamma^{2} H_{1}^{2} T_{1} T_{2}} \\
& \chi^{\prime \prime}=\frac{\omega_{o} \chi_{o} T_{2}}{1+T_{2}^{2}\left(\omega_{o}-\omega\right)^{2}+\gamma^{2} H_{1}^{2} T_{1} T_{2}}
\end{aligned}
$$

In the denominator the factor $\gamma^{2} \mathrm{H}_{1}{ }^{2} \mathrm{~T}_{1} \mathrm{~T}_{2}$ is called the saturation factor which can be made $<<1$ by choosing $\mathrm{H}_{1}{ }^{2}$ small provided $\mathrm{T}_{1}$ and $\mathrm{T}_{2}$ are not too large. 
In magnetic resonance experiments, the power $\mathrm{P}$ absorbed by the sample is measured as either the magnetic field $\mathrm{H}$ is varied (EMR or ESR) or the frequency $\omega$ is varied (NMR) through the resonance condition $\omega_{0}=\gamma \mathrm{H}_{0}$. The power absorbed can be written as:

$$
P=\frac{1}{\tau} \int_{0}^{\tau}\left(H \cdot \frac{d M}{d t}\right) d t, \tau=\frac{2 \pi}{\omega}
$$

For $\mathrm{H}_{\mathrm{x}}=\mathrm{H}_{1} \cos (\omega \mathrm{t})$, Eq. 4.10 can be written as:

$$
\begin{aligned}
P & =\frac{1}{2 \tau} \int_{0}^{\tau} H_{1}^{2} \omega\left(\chi^{\prime \prime}(1+\cos (2 \omega t))-\chi^{\prime} \sin (2 \omega t)\right) d t \\
& =\frac{1}{2} H_{1}^{2} \omega \chi^{\prime \prime}
\end{aligned}
$$

Substituting for $\chi^{\prime \prime}$ in Eq. 4.11 under the condition $\mathrm{S}=\gamma^{2} \mathrm{H}_{1}{ }^{2} \mathrm{~T}_{1} \mathrm{~T}_{2}<<1$ leads to:

$$
P=\frac{1}{2} \frac{H_{1}^{2} \omega \omega_{o} \chi_{o} \Delta \omega_{o}}{\left(\omega_{o}-\omega\right)^{2}+(\Delta \omega)^{2}}
$$

where $\Delta \omega=1 / T_{2}$ is the linewidth in the frequency units. Thus the linewidth is a measure of $\mathrm{T}_{2}$, the spin-spin relaxation time under the condition that $\mathrm{S}<<1$ and there is no explicit interaction between the magnetic moments. The spin-lattice relaxation time $T_{1}$ is often measured by increasing $\mathrm{H}_{1}$ so that the saturation factor $\mathrm{S}=1$. In all our experiments $\mathrm{H}_{1}$ was small enough so that $\mathrm{S}<<1$. From Eq. 4.12 , it is evident that $\mathrm{P}$ is also directly proportional to $\chi_{0}$, the static susceptibility and the lineshape for $\mathrm{P}$ is Lorentzian with the linewidth $\Delta \mathrm{H}=\Delta \omega / \gamma$ and the resonance field $\mathrm{H}_{\mathrm{o}}=\omega_{0} / \gamma$ in EMR.

\subsection{Magnetic Resonance in Anisotropic Systems}

If $\mathrm{H}_{\mathrm{A}}$ is the magnetocrystalline anisotropy along the z-axis, and $\mathrm{H}_{\mathrm{o}}$ is applied along the anisotropy axis $\mathrm{H}_{\mathrm{o}}=\mathrm{H}_{\mathrm{o}} \hat{k}$, then the total $\mathrm{H}$ is given by:

$$
\begin{aligned}
& H=\left(H_{o}+H_{A}\right) \hat{k} \\
& \omega_{o}=\gamma\left(H_{o}+H_{A}\right)
\end{aligned}
$$


or

$$
H_{o}=\left(\frac{\omega_{o}}{\gamma}\right)-H_{A}
$$

According to Eq. 4.15, the resonance field $\mathrm{H}_{\mathrm{o}}$ at a fixed frequency $\omega_{\mathrm{o}}$ shifts to lower fields by the amount equal to $\mathrm{H}_{\mathrm{A}}$. This equation is used in the interpretation of the data on Ni nanoparticles.

Another source that shifts the resonance field from $\mathrm{H}_{\mathrm{o}}$ is the effect of demagnetizing fields in non-spherical particles. Kittel has derived the equations for resonance fields in the presence of demagnetizing fields leading to the effective field:

$$
H=H_{o} \hat{k}-N_{i} M_{i}
$$

where $\mathrm{i}=\mathrm{x}, \mathrm{y}$, $\mathrm{z}$ leads to demagnetization fields of $\mathrm{N}_{\mathrm{x}} \mathrm{M}_{\mathrm{x}}, \mathrm{N}_{\mathrm{y}} \mathrm{M}_{\mathrm{y}}, \mathrm{N}_{\mathrm{z}} \mathrm{M}_{\mathrm{z}}$ along the three axis of an ellipsoidal shaped sample. The general resonance condition in this case is given by [Kittel, 1996]:

$$
\left(\frac{\omega_{o}}{\gamma}\right)^{2}=\left(H_{o}+\left(N_{y}-N_{z}\right) M_{o}\right)\left(H_{o}+\left(N_{x}-N_{z}\right) M_{o}\right)
$$

For a sphere, $\mathrm{N}_{\mathrm{x}}=\mathrm{N}_{\mathrm{y}}=\mathrm{N}_{\mathrm{z}}=4 \pi / 3$ reducing Eq. 4.17 to $\left(\omega_{\mathrm{o}} / \gamma\right)=\mathrm{H}_{\mathrm{o}}$.

On the other hand, for a thin film with $\mathrm{H}_{\mathrm{o}} \hat{z}$ in the film plane, $\mathrm{N}_{\mathrm{z}}=\mathrm{N}_{\mathrm{y}}=0$ and $\mathrm{N}_{\mathrm{x}}$ $=4 \pi$ for perpendicular to the film. Substituting these in Eq. 4.17 leads to:

$$
\left(\frac{\omega_{o}}{\gamma}\right)^{2}=H_{o}\left(H_{o}+4 \pi M_{o}\right)
$$

Eq. 4.18 yields $\mathrm{H}_{\mathrm{o}}$ shifted to lower fields because of demagnetizing factor. Similarly, for $H_{o} \hat{Z}$ perpendicular to the film, $\mathrm{N}_{\mathrm{z}}=4 \pi$ and $\mathrm{N}_{\mathrm{x}}=\mathrm{N}_{\mathrm{y}}=0$ yielding:

$$
\left(\frac{\omega_{o}}{\gamma}\right)=H_{o}-4 \pi M_{o} \text { or } H_{o}=\left(\frac{\omega_{o}}{\gamma}\right)+4 \pi M_{o}
$$

Therefore the resonance field is shifted to higher fields. These consideration are used later to explain the shifts of $\mathrm{H}_{\mathrm{o}}$ observed in $\mathrm{Ni}$ nanoparticles. 


\subsection{AC Susceptibility Theory}

In studying the dynamics of a system all systems that exhibit hysteresis undergo relaxation process to return to their thermodynamic equilibrium position. Here we will begin with the basic equation [Chudnovsky et al, 1998]:

$$
\tau\left(\frac{d M}{d t}\right)+M=0
$$

which has been derived from $M=M_{o} e^{-\frac{t}{\tau}}$ for the moments that relax to their equilibrium position as a function of time. In the presence of an oscillating field $h=h_{o} \cos (\omega t)$ the above equation becomes:

$$
\tau\left(\frac{d M}{d t}\right)+M=\chi_{o} h_{o} \cos (\omega t)
$$

If

$$
M=\chi^{\prime} h_{o} \cos (\omega t)+\chi \chi^{\prime \prime} h_{o} \sin (\omega t)
$$

and we differentiate Eq. 4.21 and multiply by $\tau$ we obtain the following equation:

$$
\tau^{2}\left(\frac{d^{2} M}{d t^{2}}\right)+\tau\left(\frac{d M}{d t}\right)=-\omega \tau \chi_{o} h_{o} \sin (\omega t)
$$

Upon calculating the second derivative of Eq. 4.22 and substituting it along with Eq. 4.21 in Eq. 4.23 we get:

$$
M=\frac{\chi_{o} h_{o}}{1+\omega^{2} \tau^{2}}(\cos (\omega t)+\omega t \sin (\omega t))
$$

If we compare this equation with Eq. 4.22 we obtain $\chi^{\prime}$ and $\chi^{\prime \prime}$ as:

$$
\begin{aligned}
& \chi^{\prime}=\frac{\chi_{o}}{1+\omega^{2} \tau^{2}} \\
& \chi^{\prime \prime}=\frac{\omega \tau \chi_{o}}{1+\omega^{2} \tau^{2}}
\end{aligned}
$$


For non-interacting magnetic NPs with volume V and anisotropy K, Andersson et al have derived $\chi^{\prime}$ and $\chi^{\prime \prime}$ for a randomly oriented case. $\chi^{\prime}$ and $\chi^{\prime \prime}$ defined as the in-phase and out-of-phase components of the AC susceptibility from considering the moment of a particle in the presence of a field with the particles easy axis distributed randomly. Here we start from the basic equation for the energy of a particle given by [Andersson et al, 1997]:

$$
E_{i}=-K V_{i}\left(\hat{\mu}_{i} \cdot \hat{n}_{i}\right)^{2}-M_{S} V_{i} \hat{\mu}_{i} \cdot \vec{B}_{i}
$$

The first term in the energy comes from the anisotropy that arises from the moment not lying along the direction of the easy magnetization axis. The second term is the Zeeman term that comes from the magnetic moment placed in an external magnetic field, $K$ defines the anisotropy constant, $V_{i}$ is the particle volume, $M_{S}$ is the saturation magnetization and $H_{a}$ the applied field with $\vec{B}_{i}=\mu_{0} H_{a} \hat{z}$. The details of the calculation are described in appendix II. Described below is the summary of the derivation that originates from the paper by Andersson et al. The energy relation can be also expressed as:

$$
E_{i}=-K V_{i}\left[\cos ^{2}\left(\alpha_{i}\right)+\left(\frac{M_{S}}{K}\right)\left|\vec{B}_{i}\right| \cos \left(\psi_{i}-\alpha_{i}\right)\right]
$$

where angle $\Psi_{\mathrm{i}}$ is between the easy magnetization axis and the external field and the angle $\alpha_{i}$ between the magnetic moment and the easy magnetization axis. We proceed by minimizing Eq. 4.27 with respect to the angle $\alpha_{\mathrm{I}}$ and obtain the following:

$$
2 \sin \alpha_{i} \cos \alpha_{i}=x\left[\sin \left(\psi_{i}\right) \cos \left(\alpha_{i}\right)-\cos \left(\psi_{i}\right) \sin \alpha_{i}\right] \quad x=\frac{M_{S}}{K}\left|\vec{B}_{i}\right|=\frac{M_{S}}{K}\left(\mu_{0} H_{a}\right)
$$


Field perpendicular to the easy axis of magnetization

Case I

When the magnetic field is perpendicular to the easy axis $\cos \left(\psi_{i}\right)=0$, and the moment lies in the direction of the easy axis $\sin \left(\alpha_{i}\right)=\alpha_{i}, \cos \left(\alpha_{i}\right)=1$ we get $\alpha_{1}=\frac{x}{2} \sin (\psi)$ and the energy corresponding to this angle is:

$$
E_{1}=-K V[1+x \cos (\psi)] \text { for } \psi_{i}=90
$$

Case II

For $\alpha_{2}=\pi-\frac{x}{2} \sin (\psi)$ the energy is given by:

$$
E_{2}=-K V[1-x \cos (\psi)] \text { for } \psi_{i}=270
$$

Field along the easy axis of magnetization

When the magnetic field is along the easy axis $\sin \left(\psi_{i}\right)=0$, and the moment lies in the direction of the easy axis, we get $\alpha_{S}=\frac{\pi+x \cos (\psi)}{2}$ and the energy corresponding to this angle is:

$$
E_{S}=-K V x \sin (\psi)
$$

We can obtain the z-component of the particle magnetization along the direction of the external field from $m_{1}=M_{S} \cos \left(\psi-\alpha_{1}\right)$ where for $\alpha_{1}=\frac{x}{2} \sin (\psi)$ we get:

$$
m_{1} \approx M_{S}\left(\cos (\psi)+\sin ^{2}(\psi) \frac{x}{2}\right)
$$

and $m_{2}=M_{S} \cos \left(\psi-\alpha_{2}\right)$ where for $\alpha_{2}=\pi-\frac{x}{2} \sin (\psi)$ we get:

$$
m_{2} \approx M_{S}\left(-\cos (\psi)+\frac{x}{2} \sin ^{2}(\psi)\right)
$$


We will now apply the Néel-Arrhenius relation for the moments that lie along the saddle point and have to cross the energy barrier with the same attempt frequency and align along the direction of the magnetic field. The relation for those particles is given by:

$$
\begin{gathered}
\tau=\frac{\tau_{0}}{e^{-\left(E_{S}-E_{1}\right) / k_{B} T}+e^{-\left(E_{S}-E_{2}\right) / k_{B} T}} \\
\tau=\frac{\tau_{0}}{-(-K V x \sin \psi-(-K V(1+x \cos \psi))) / k_{B}{ }^{T}+(-K V x \sin \psi-(-K V(1-x \cos \psi))) / k_{B} T}
\end{gathered}
$$

where we have substituted for $\mathrm{E}_{1}, \mathrm{E}_{2}$ and $\mathrm{E}_{\mathrm{S}}$. In the low-x limit the above relation reduces to $\tau=\frac{\tau_{0}}{2} e^{K V / k_{B} T}$.

The fraction of moments that lie in the $\mathrm{m}_{1}$ direction are given by $n^{+}(t)=n_{e q}+\left(\frac{1}{2}-n_{e q}\right) P(t)$ where $P(t)=e^{-t / \tau}$ denotes that the magnetization has not switched after a time $t$ and $n_{e q}=\frac{1}{1+e^{-\left(E_{2}-E_{1}\right) / k_{B} T}}$ where when $E_{1}=E_{2}, n_{e q}=\frac{1}{2}$. The value for $\mathrm{n}_{\mathrm{eq}}$ used is $n_{e q} \approx \frac{1}{2}\left(1+\frac{K V x \cos \psi}{k_{B} T}\right)$ where the expansion for $\mathrm{e}^{\mathrm{x}}$ was used. The magnetization then follows the relation given by:

$$
\begin{gathered}
m(t)=n^{+}(t) m_{1}+\left(1-n^{+}(t)\right) m_{2} \\
m(t)=\frac{\mu_{0} M_{S}^{2} H_{a}}{K}\left[\cos ^{2}(\psi) \frac{K V}{k_{B} T}\left(1-e^{-t / \tau}\right)+\frac{1}{2} \sin ^{2}(\psi)\right]
\end{gathered}
$$

If we average over $\psi$ assuming random particle easy axis then eq. (4.37b) reduces to

$$
m(t)=\left[\frac{\mu_{0} M_{S}^{2} H}{K}\left[\frac{1}{3} \frac{K V}{k_{B} T}\left(1-e^{-t / \tau}\right)+\frac{1}{3}\right]\right]
$$

giving $\chi(t)=\frac{m(t)}{H_{a}}=\frac{\mu_{0} M^{2} s}{3 K}\left[\frac{K V}{k_{B} T}\left(1-e^{-t / \tau}\right)+1\right]$ 
The AC susceptibility is given by $\chi(\omega)=\int_{0}^{\infty} \frac{d \chi}{d t} e^{-i \omega t} d t$ where upon differentiation we obtain $\frac{d \chi}{d t}=\frac{\mu_{0} M_{S}^{2}}{3 K}\left[\delta(t)+\frac{K V}{k_{B} T \tau} e^{-t / \tau}\right]$

The presence of a delta function comes from the fact that the two energy minima shift towards the direction of the applied field as soon as the field is applied. This gives rise to net magnetization which leads to a non-zero contribution to the susceptibility. We can integrate this relation below to yield:

$$
\chi(\omega)=\frac{\mu_{0} M^{2}{ }_{s}}{3 K} \int_{0}^{\infty}\left(\delta(t)+\frac{K V}{k_{B} T \tau} e^{-t / \tau}\right) e^{-i \omega t} d t
$$

to obtain

$$
\chi(\omega)=\frac{\mu_{0} M^{2}{ }_{s}}{3 K}\left[1+\frac{K V}{k_{B} T} \frac{1}{1+(\omega \tau)^{2}}-i \frac{K V}{k_{B} T} \frac{\omega \tau}{1+(\omega \tau)^{2}}\right]
$$

Upon comparing the above relation with $\chi(\omega)=\chi^{\prime}-i \chi^{\prime \prime}$ the in-phase and out-of-phase susceptibilities can be obtained as:

$$
\begin{aligned}
& \chi^{\prime}=\frac{\mu_{0} M^{2} s}{3 K}\left[1+\frac{K V}{k_{B} T} \frac{1}{1+(\omega \tau)^{2}}\right] \\
& \chi^{\prime \prime}=\frac{\mu_{0} M^{2}{ }_{s}}{3 K}\left[\frac{K V}{k_{B} T} \frac{\omega \tau}{1+(\omega \tau)^{2}}\right]
\end{aligned}
$$

Eqs. 4.42 when compared with Eqs. 4.25 gives:

$$
\chi_{o}=\frac{\mu_{0} M^{2}{ }_{S} V}{3 k_{B} T}
$$

where Eqs. 4.42 becomes:

$$
\begin{aligned}
& \chi^{\prime}=\text { const }+\chi_{o} \frac{\omega \tau}{1+\omega^{2} \tau^{2}} \\
& \chi^{\prime \prime}=\frac{\chi_{o} \omega \tau}{1+\omega^{2} \tau^{2}}
\end{aligned}
$$

In chapter 5 , these equations are used to simulate $\chi$ and $\chi^{\prime \prime}$ and compare with the experimental results in Ni NPs. 


\section{CHAPTER V}

\section{Experimental results from SQUID magnetometry and discussion}

\subsection{SQUID Magnetometry}

The magnetic measurements were done on a commercial superconducting quantum interference device (SQUID) magnetometer in the Reciprocating Sample Option (RSO) mode. The RSO mode measures a sample by moving it rapidly and sinusoidally through the SQUID pickup coils. The RSO transport moves up and down oscillating the sample around the measurement position. When the sample moves through the coils, the SQUID response to the magnetic moment is measured. This is done for a specified number of cycles till the data is fit to the raw SQUID voltage data. It is done repeatedly till the Magnetic Property Measurement System (MPMS) MultiVu calculates the average sample moment from all the fits which are then stored in the active data files.

The other measurement using the RSO mode is the AC susceptibility which is a two-point measurement. In this measurement, the sample is positioned at the positively oriented bottom pick up coil and then the system applies an oscillating AC magnetic field to the sample. This $\mathrm{AC}$ response is monitored and the system calculates a nulling waveform which will cancel this response. This is sent back to the SQUID feedback circuit where the AC SQUID response is recorded. This iterative calculation of the nulling waveform continues till the amplitude of the wave iteration is smaller than the null amplitude. Once the nulling is complete the MPMS MultiVu measures the remnant signal $M_{b}$ which is fit to the equation $M_{b}=A+B t+M^{\prime} \cos (\omega t)+M^{\prime \prime} \sin (\omega t)$ where $A$ is $D C$ offset, B is linear drift in field or temperature, $\omega$ is angular frequency of the AC drive signal and $\mathrm{M}^{\prime}$ and $\mathrm{M}^{\prime \prime}$ are proportional to in-phase and out-of-phase components that are still present from drive nulling procedure.

The sample now gets positioned at the center of the two middle negatively oriented pickup coils. The same AC drive signal and the nulling waveform that were applied at the bottom pickup coils are now applied here. The nulling signal still cancels the AC drive signal, however the AC signal generated by the sample changes polarity and the component of the nulling signal that nulled the samples response at the bottom coil now adds constructively to the sample signal, to create AC susceptibility sine wave with 
triple amplitude. MPMS again measures the remnant signal $\mathrm{M}_{\mathrm{C}}$ and the data is fit to $M_{C}=A+B t+M^{\prime} \cos (\omega t)+M^{\prime \prime} \sin (\omega t)$. The two sets of data are in the form $M_{b}=M f(b)+M_{o}$ and $M_{C}=\operatorname{Mf}(c)+M_{o}$ where $M$ is the actual moment of the sample, $M_{o}$ is the residual signal and $\mathrm{f}(\mathrm{x})$ is the normalized response function of the magnetometer to an idealized dipole at position $\mathrm{x}$. This function is determined in a separate measurement using the DC superconducting magnet. The components of the susceptibility can then be calculated from the difference between the two measurements. The importance of two point measurements is the elimination of the residual part from the data [Quantum Design, 1999].

\subsection{Blocking Temperature and Particle Size}

The measurements were done on the SQUID where the samples were weighed and were placed in the center of the sample holder which was a white drinking straw with a diamagnetic susceptibility of $-2.3 \times 10^{-8} \mathrm{emu}$. The straw with the sample was attached to the end of a long rod and lowered into the SQUID chamber. The measurements conditions were then controlled by the software. The data was then corrected for the background using the relation:

so

$$
\mathrm{M}_{\text {measured }}=\mathrm{M}_{\text {sample }}+\mathrm{M}_{\text {background(straw) }}
$$

or

$$
\begin{aligned}
& \mathrm{M}_{\text {sample }}=\mathrm{M}_{\text {measured }}-\mathrm{M}_{\text {straw }} \\
& \mathrm{M}_{\text {sample }}=\mathrm{M}_{\text {measured }}+2.3 \times 10^{-8} \mathrm{H} .
\end{aligned}
$$

Here $\mathrm{H}$ is the applied field in Oe. Finally, the data was corrected for the concentrations of $\mathrm{Ni}$ in the $\mathrm{Ni} / \mathrm{SiO}_{2}$ sample since $\mathrm{SiO}_{2}$ is only a weak diamagnet.

Field cooled (FC) and zero field cooled (ZFC) curves were obtained on the samples on the following way. In order to obtain the ZFC curve, the sample was cooled to $2 \mathrm{~K}$ from room temperature in the absence of an external field. Upon reaching $2 \mathrm{~K}$ the field was turned on and the moment of the sample vs. temperature was measured till room temperature. The field was kept on during this entire time and the sample was cooled from room temperature to $2 \mathrm{~K}$ with the moment vs. temperature being measured. This corresponds to the FC curve.

Fig. 5.1 below shows the $\chi$ vs. $\mathrm{T}$ data for the 1,5 and $15 \% \mathrm{Ni} / \mathrm{SiO}_{2}$ samples annealed at $\mathrm{T}_{\mathrm{a}}=400^{\circ}$ and $800^{\circ} \mathrm{C}$ in an applied filed of $\mathrm{H}=100$ Oe. Blocking temperature 
$\mathrm{T}_{\mathrm{B}}$ is obtained from the peaks in the $\chi$ vs. T data for the zero-field cooled (ZFC) case. From Fig. 5.1 it is evident that there is no definite peak in $\chi(\mathrm{ZFC})$ for any of the samples except for the $15 \% \mathrm{Ni} / \mathrm{SiO}_{2}$ at $\mathrm{T}_{\mathrm{a}}=400^{\circ} \mathrm{C}$, suggesting that there is a distribution of blocking temperatures presumably resulting from the distribution in particles sizes. For these reasons, most of the remaining studies reported here were carried out on the $15 \%$ $\mathrm{Ni} / \mathrm{SiO}_{2}$ samples only, annealed at temperatures between $400^{\circ} \mathrm{C}$ and $800^{\circ} \mathrm{C}$ to obtain different size particles as described in chapter 2 .
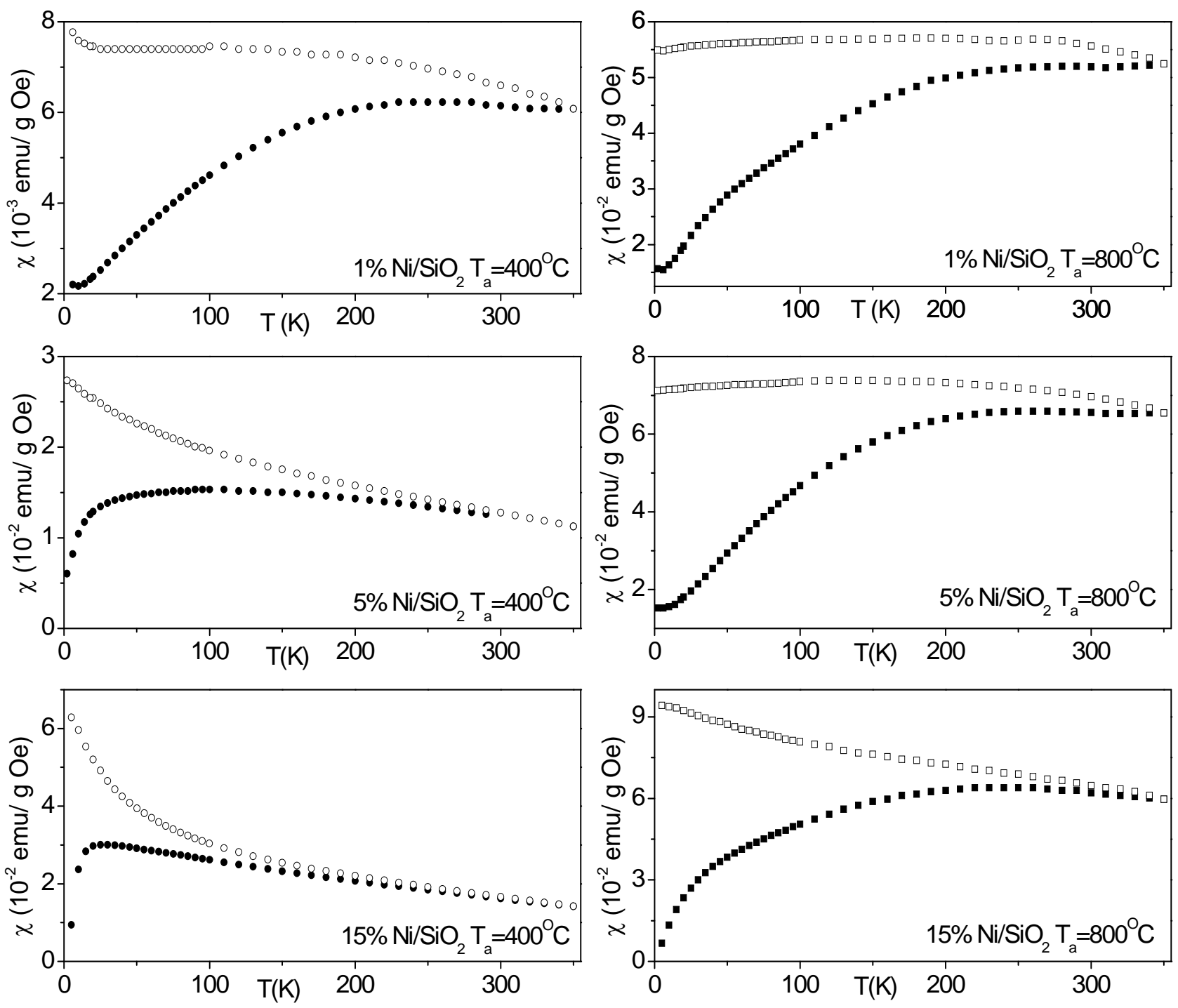

Fig. $5.1 \chi$ vs. $\mathrm{T}$ data for 1,5 and $15 \% \mathrm{Ni} / \mathrm{SiO}_{2}$ samples annealed at $\mathrm{T}_{\mathrm{a}}=400$ and $800^{\circ} \mathrm{C}$ in $\mathrm{H}=100 \mathrm{Oe}$. The open symbols are for the $\mathrm{FC}$ data and closed symbols are for the ZFC data. 


\subsection{Magnetic Field Dependence of the Blocking Temperature}

In Fig. 5.2 (a, b, c, d, e) temperature dependence of $\chi$ for the five samples investigated in this work with $\mathrm{D}=3.8,11.7,15,21$ and $23 \mathrm{~nm}$ are displayed for two applied fields $\mathrm{H}=100$ Oe and $\mathrm{H}=1000$ Oe. In all cases, the peak temperature for $\chi(\mathrm{ZFC})$ curve shifts to lower temperatures as field is increased. This decrease in $T_{B}$ with increase in $\mathrm{H}$ was investigated in more detail for the $\mathrm{D}=3.8 \mathrm{~nm}$ particles and published in a recent paper [Singh et al, 2008]. The plot of the data for $\chi$ vs. T for the $3.8 \mathrm{~nm}$ particles for different fields is shown in Fig. 5.3. $\mathrm{T}_{\mathrm{B}}$ measured by peaks in $\chi(\mathrm{ZFC})$ are plotted against $\mathrm{H}$ in Fig. 5.4. This data is fit to the theoretically expected equation [Victora, 1989, Walton, 1990]:

$$
T_{B}(H)=T_{B 0}\left[1-\frac{H}{H_{0}}\right]^{m}
$$

with predicted variations according to $m=2$ and $m=3 / 2$ are represented by solid lines in Fig. 5.4. The data fits with $m=2$ variation for lower $H$ and $m=3 / 2$ variation for higher fields with $\mathrm{H}_{\mathrm{o}}=3200 \mathrm{Oe}$ and $\mathrm{T}_{\mathrm{Bo}}=33 \mathrm{~K}$. Theoretical arguments suggest $\mathrm{m}=2$-r variation with $\mathrm{r}=0$ to 0.6 depending on the angle $\theta$ between $\mathrm{H}$ and the anisotropy (easy) axis of the NP [Victora, 1989, Walton, 1990]. The result shown in Fig. 5.4 confirm this since $\mathrm{m}$ is neither strictly 2 nor $3 / 2$ over the whole range of $H$. This is reasonable since $\theta$ is likely different for different particles in a powder sample.

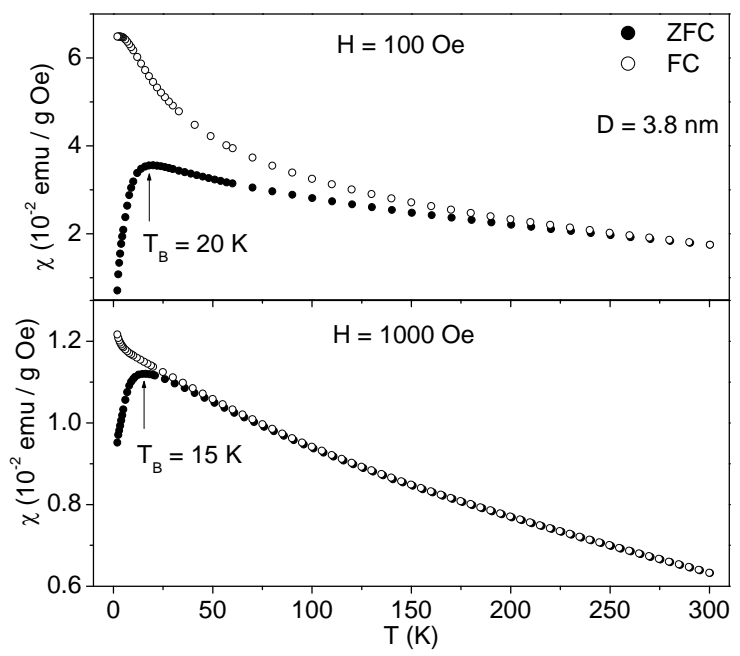

(a)

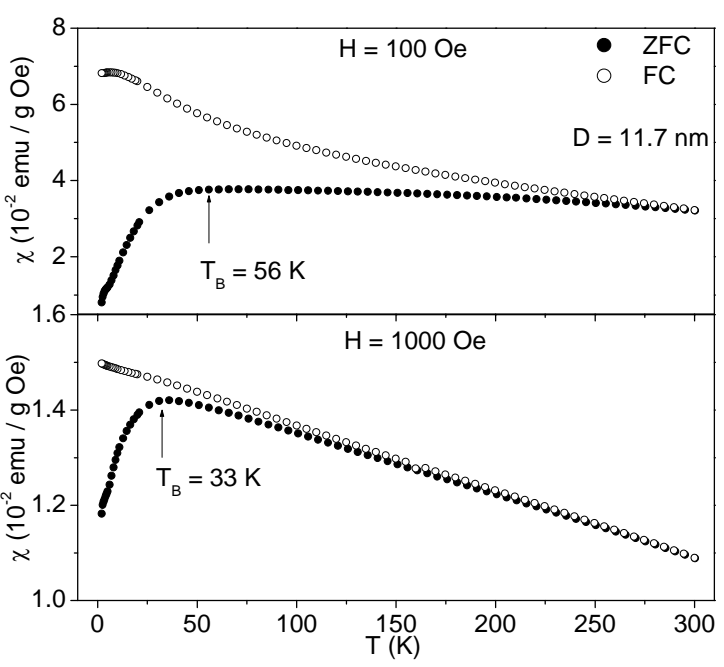

(b) 


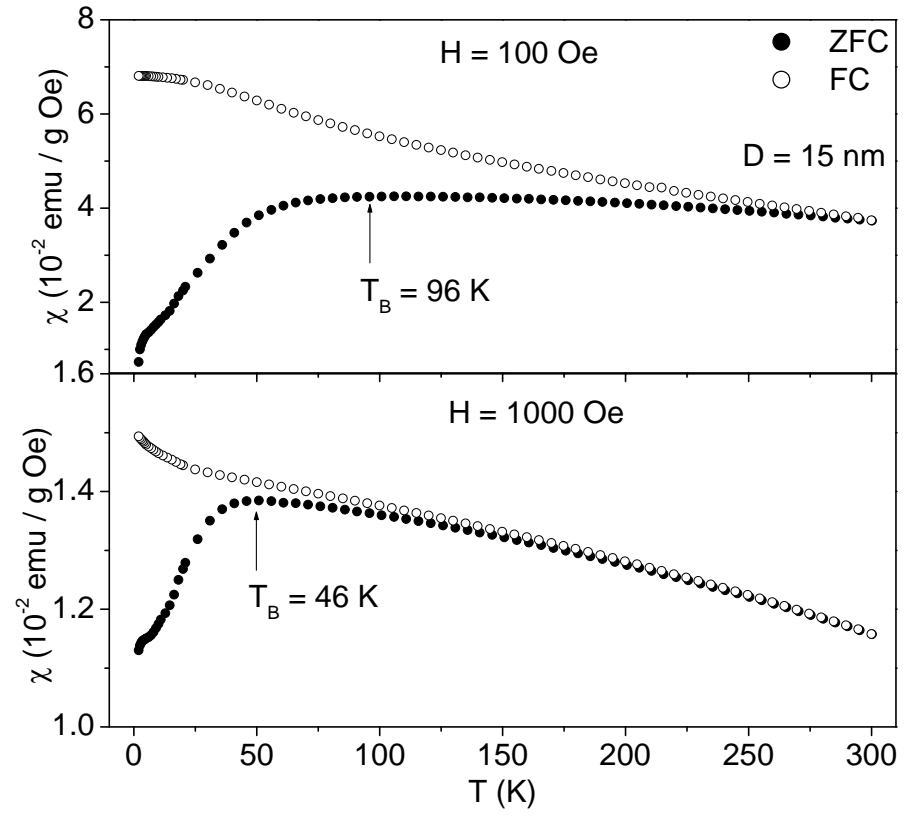

(c)

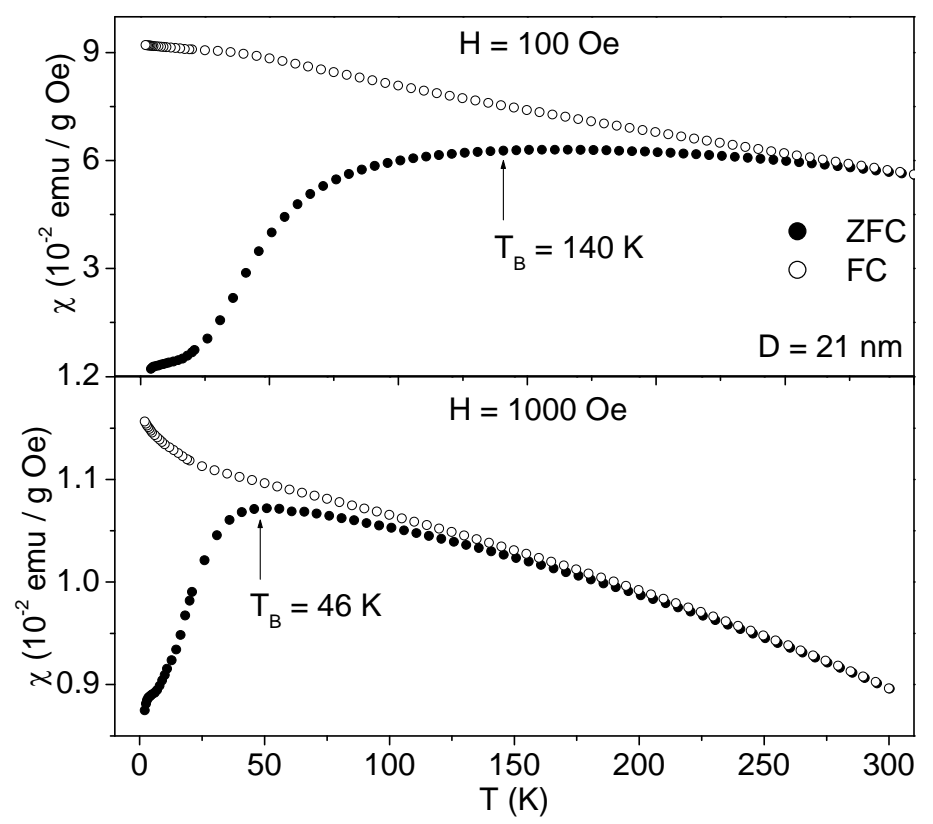

(d)

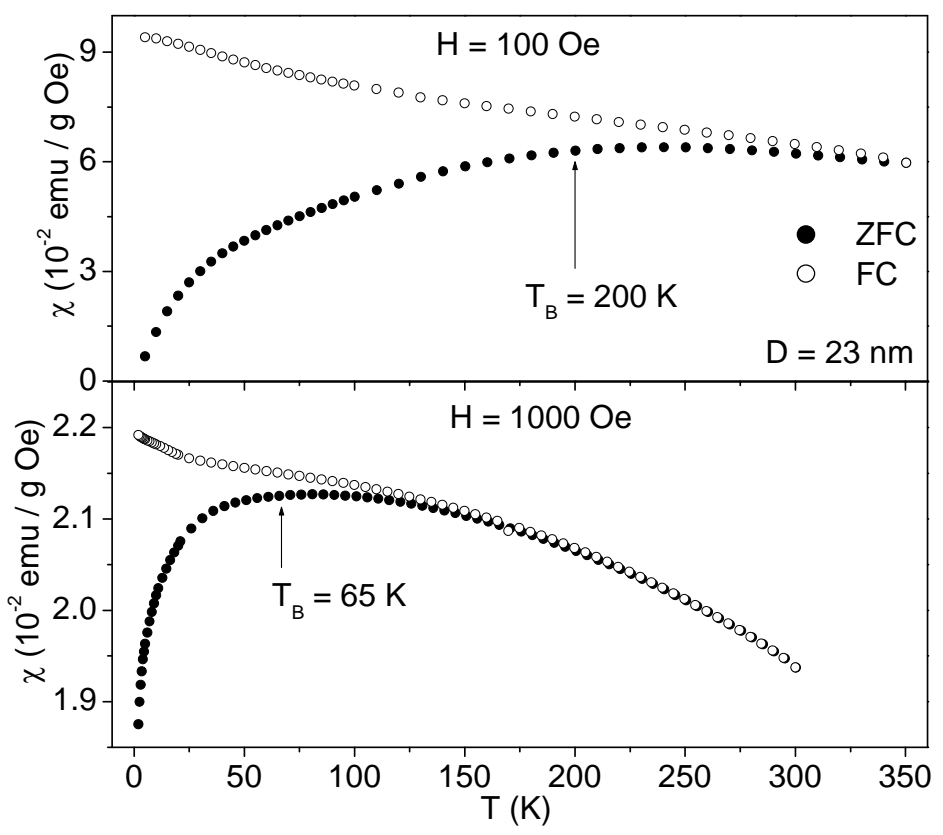

(e)

Fig. $5.2 \chi$ vs. T plots for particles sizes 3.8, 11.7, 15, 21 and $23 \mathrm{~nm}$ showing decrease in blocking temperature with an increase in applied field. 

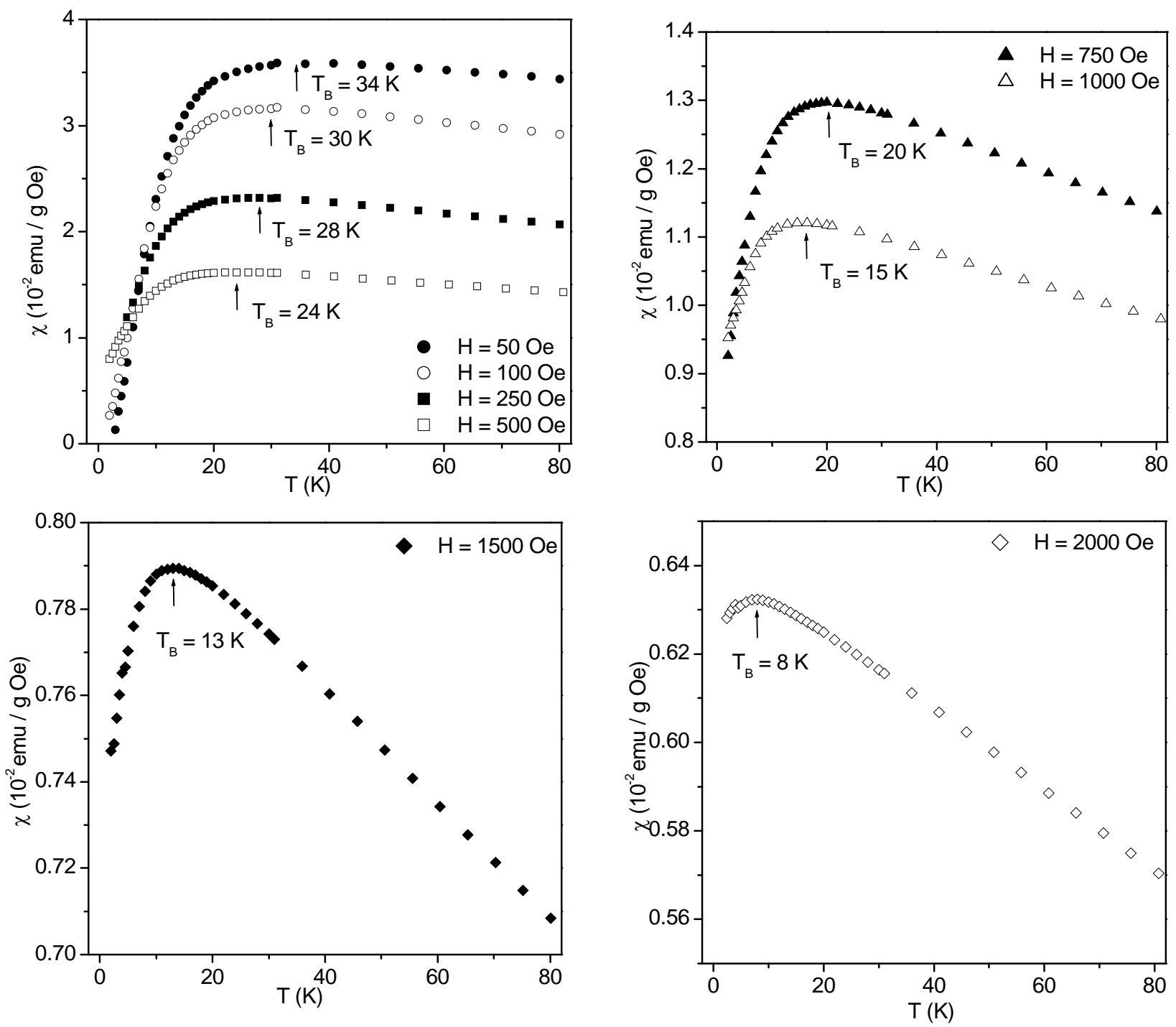

Fig. 5.3 Zero field cooled curves $\chi$ vs. T for the applied field varying from $\mathrm{H}=50$ to 2000 Oe for the $3.8 \mathrm{~nm}$ nanoparticles. 


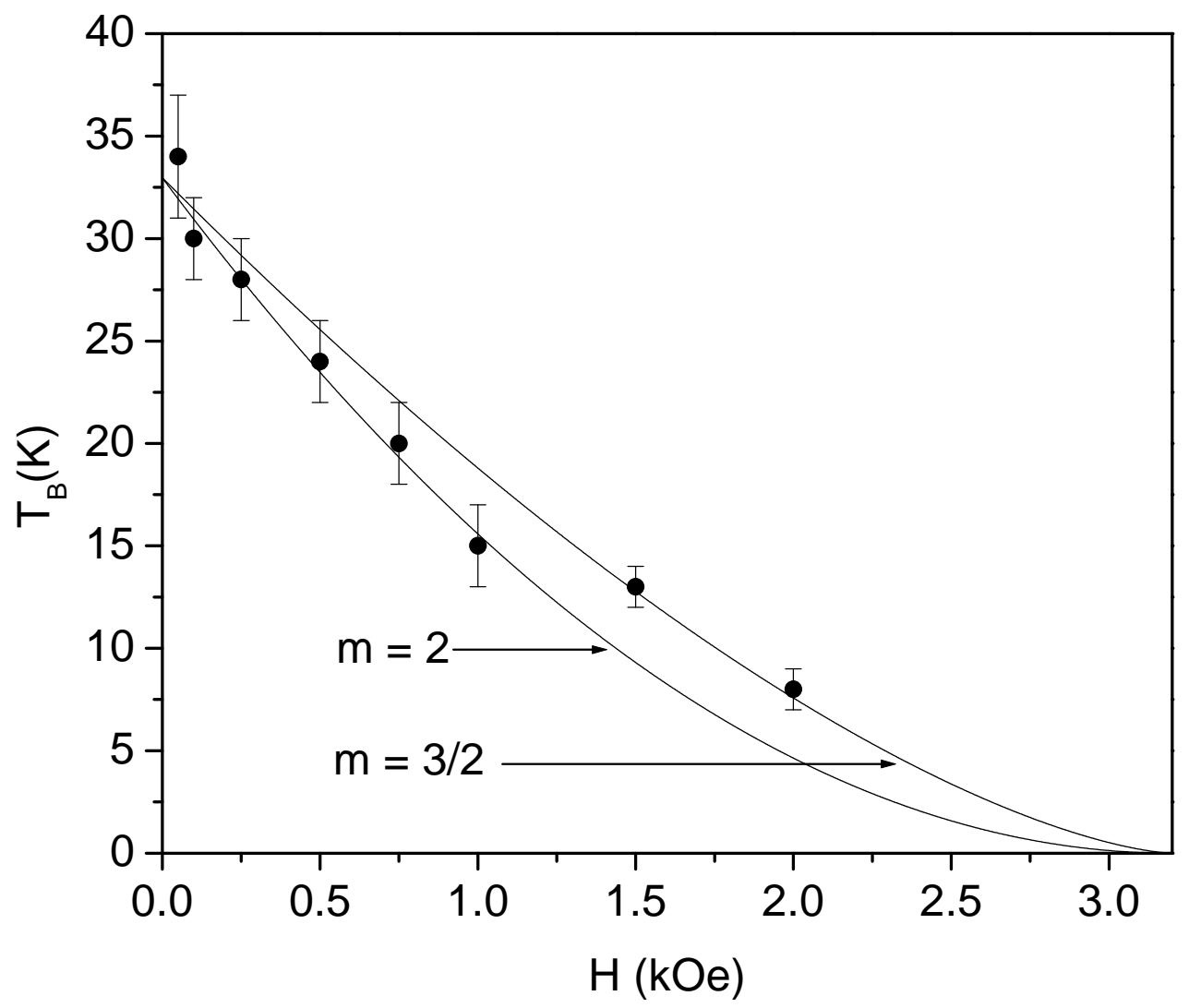

Fig. 5.4 Change in $\mathrm{T}_{\mathrm{B}}$ with applied field $\mathrm{H}$, with the solid lines as fits to Eq. 5.1 for $m=2$ and $m=3 / 2$. 


\subsection{Langevin Function and Magnetic Moment per Particle}

One method to test whether these particles are superparamagnetic involves the measurement of magnetization as a function of temperature above $T_{B}$ where the plot of magnetization with $\mathrm{H} / \mathrm{T}$ collapses into one curve for an ideal system with a very uniform distribution of particle size. The modified langevin function is used to determine magnetic moment per particle $\left(\mu_{\mathrm{P}}\right)$ by fitting to:

$$
\begin{aligned}
M & =M_{o} \mathcal{L}\left(\frac{\mu_{p} H}{k_{B} T}\right)+\chi_{a} H \\
\mathcal{L}(x) & =\operatorname{coth}(x)-\frac{1}{x}
\end{aligned}
$$

or equivalently by plotting $\left(\mathrm{M}-\chi_{\mathrm{a}} \mathrm{H}\right) / \mathrm{M}_{\mathrm{o}}$ vs. $(\mathrm{H} / \mathrm{T})$ [Seehra et al, 2004]. Fig. 5.5 shows the fit done for the $15 \% \mathrm{Ni} / \mathrm{SiO}_{2}$ for temperatures above the blocking temperature with different sizes and the inset showing the original data of $M$ vs. $H$ curves. The effective magnetic moment per particle was found to increase with size; $\mu_{\mathrm{P}}=3500(500) \mu_{\mathrm{B}}$, $7500(1500) \mu_{\mathrm{B}}, 11000(2000) \mu_{\mathrm{B}}, 25000(5000) \mu_{\mathrm{B}}$ for the $\mathrm{D}=3.8,11.7,15,21$ and $23 \mathrm{~nm}$ NPs respectively.

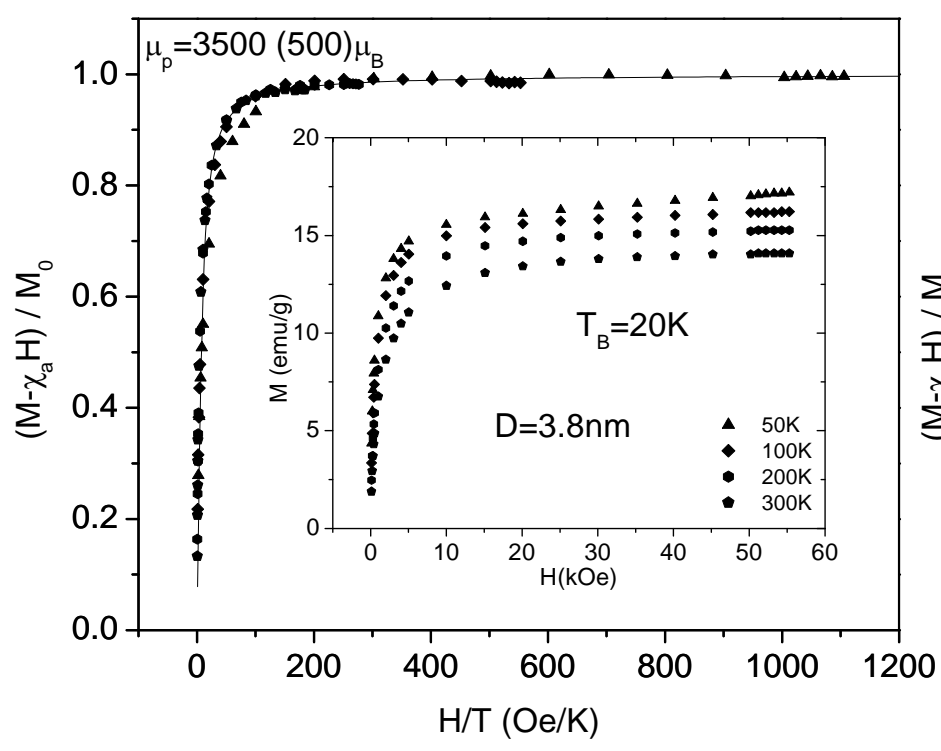

(a)

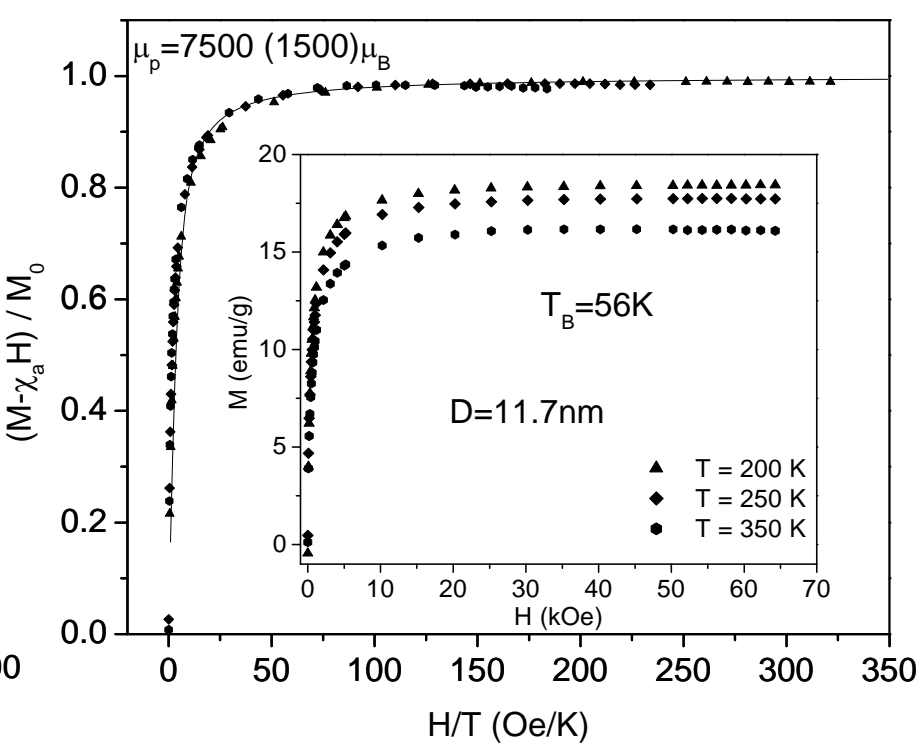

(b) 


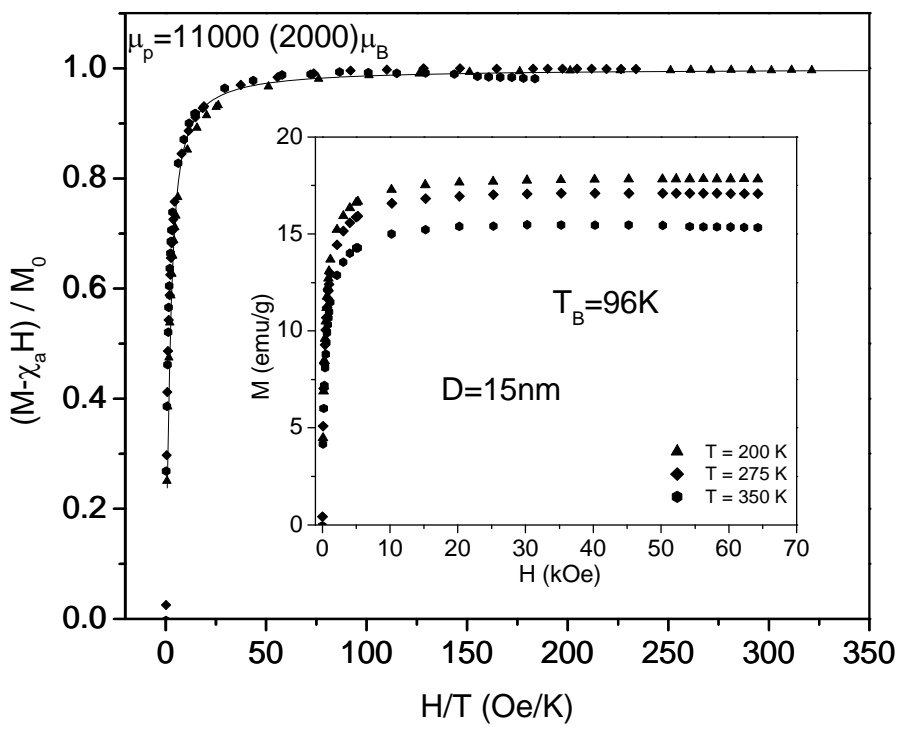

(c)

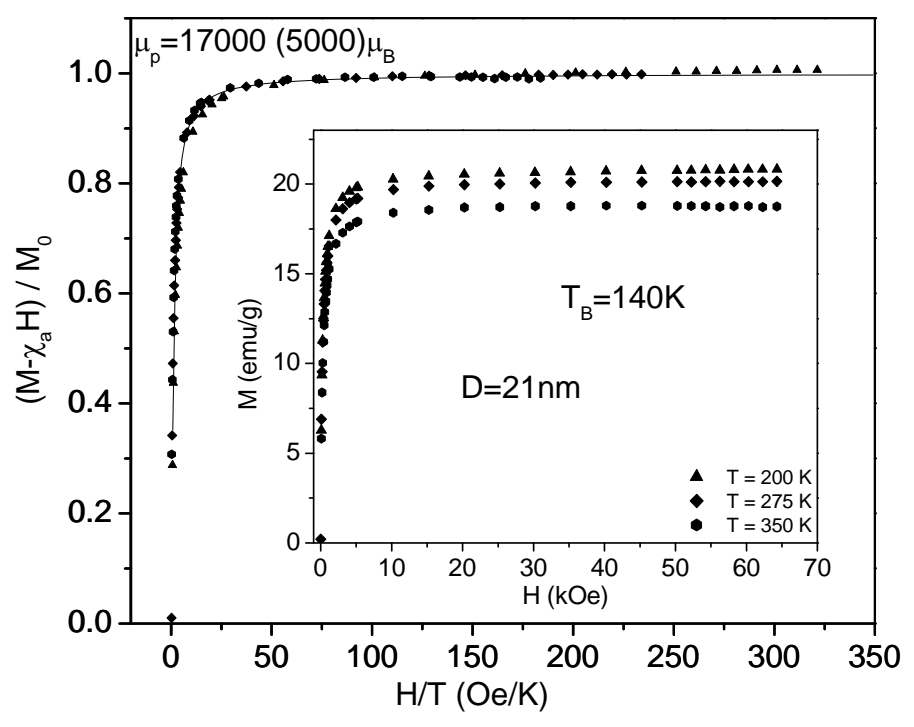

(d)

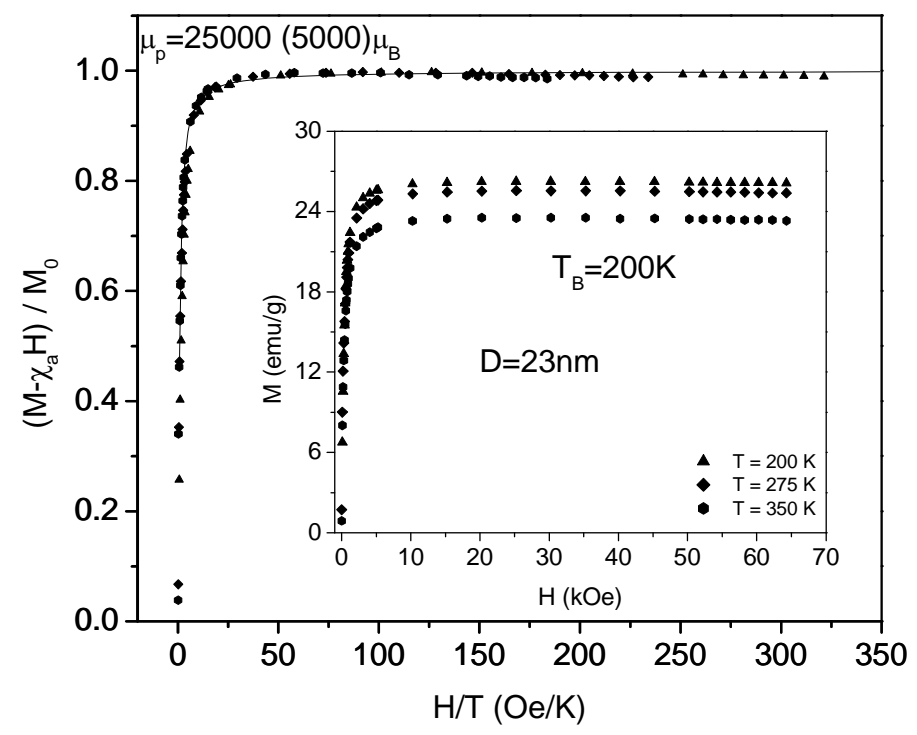

(e)

Fig. 5.5 Modified Langevin function fit (Eq. 5.2) done for the $15 \% \mathrm{Ni} / \mathrm{SiO}_{2}$ samples for $\mathrm{D}=3.8,11.7,15,21$ and $23 \mathrm{~nm}$. The inset shows the $\mathrm{M}$ vs. H plots for temperatures above the blocking temperature. 


\subsection{Variation of Moment per Particle $\left(\mu_{P}\right)$ with Particle Size (D)}

The effective magnetic moment per particle $\left(\mu_{\mathrm{P}}\right)$ is found to increase with increase in particle size D. Qualitatively, as the particle size increases, it contains more number of atoms in it and hence the magnetic moment per particle should increase. The theoretical interpretation of the variation of moment per particle with $\mathrm{D}$ is given below and the experimental results are tested thereafter.

The structure of $\mathrm{Ni}$ is face centered cubic $(\mathrm{FCC})$ with its lattice constant $(\mathrm{a}=0.352$ $\mathrm{nm})$ so the volume of the cell is given by:

$$
V_{\text {cell }}=a^{3}=\left(3.52 \times 10^{-8}\right)^{3}=4.36 \times 10^{-23} \mathrm{~cm}^{3}
$$

The cell contains 4 atoms per unit cell so the number of atoms per unit volume is:

$$
\frac{\text { No. of atoms }}{V_{\text {cell }}}=\frac{4}{4.36 \times 10^{-23}}=9.17 \times 10^{22} / \mathrm{cm}^{3}
$$

or the volume occupied by one atom is:

$$
\text { volume occupied by one atom }=\frac{1}{9.17 \times 10^{22}}=1.09 \times 10^{-23} \mathrm{~cm}^{3}
$$

The density $(\rho)$ for Ni is given by:

$$
\rho=\frac{\text { mass }}{V_{\text {cell }}}=\frac{4 \times\left(58.7 / 6.02 \times 10^{23}\right)}{4.36 \times 10^{-23}}=8.94 \mathrm{~g} / \mathrm{cm}^{3}
$$

For a spherical NP of diameter D, the volume is given by:

$$
V_{N P}=\frac{4}{3} \pi r^{3}=\frac{4}{3} \pi\left(\frac{D}{2}\right)^{3}=\frac{\pi}{6} D^{3}=0.52 D^{3} \mathrm{~cm}^{3}
$$

The number of atoms $(\mathrm{N})$ in a NP of diameter $\mathrm{D}$ is:

$$
\begin{gathered}
N=V_{N P} \times \frac{\text { No.of atoms }}{V_{\text {cell }}} \\
N=0.52 D^{3} \times 9.17 \times 10^{22}=4.77 \times 10^{22} D^{3} \text { atoms } .
\end{gathered}
$$

The magnetic moment per Ni(bulk) atom is $0.606 \mu_{\mathrm{B}}$ and hence $\mu_{\mathrm{P}}$ is given by:

$$
\mu_{P}=0.606 \mu_{B} \times N
$$


Fig. 5.6 below shows the plot of $\mu_{P}$ variation with D. The solid line is a fit to Eq. 5.10 with $\mu(\mathrm{Ni})=0.606 \mu_{\mathrm{B}}$ and $\mu(\mathrm{Ni})=0.3 \mu_{\mathrm{B}}$ observed in the nanoparticles of $\mathrm{Ni}$, as discussed later. The inset shows the experimental $\mu_{\mathrm{P}}$ vs. D. The curves deviate from the experimental value for larger D. The reasons for this discrepancy are not yet understood.

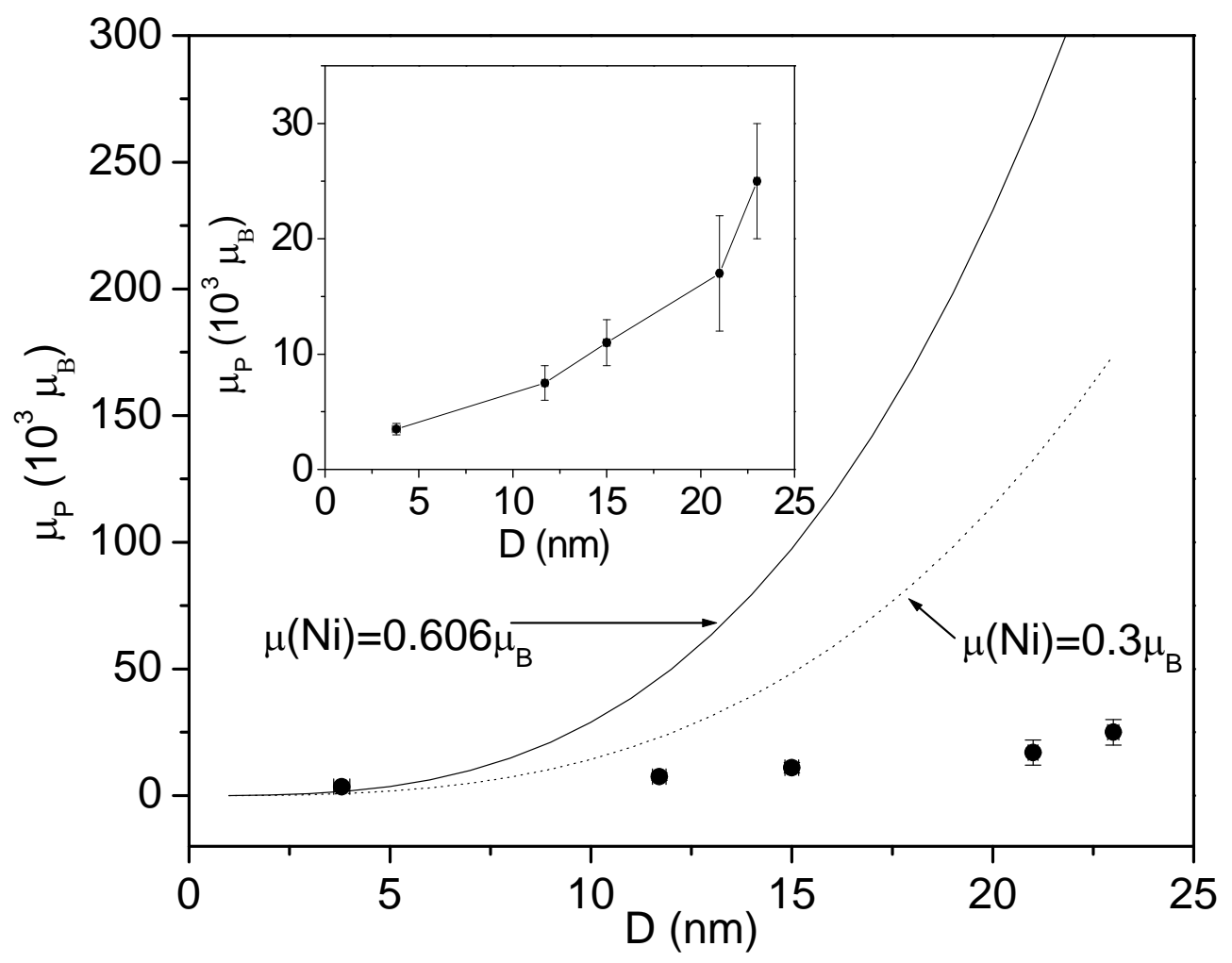

Fig. 5.6 Variation in $\mu_{\mathrm{P}}$ with $\mathrm{D}$ with the solid and dotted line as fits to Eq. 5.10 with $\mu(\mathrm{Ni})=0.606 \mu_{\mathrm{B}}$ and $\mu(\mathrm{Ni})=0.3 \mu_{\mathrm{B}}$ as discussed in text. 


\subsection{Variation of $M_{0}$ and $\chi_{a}$ with Temperature (T) and Particle Size (D)}

A. Variation of $\chi_{\mathrm{a}}$ with $\mathrm{T}$ and $\mathrm{D}$

If the magnetization curve does not saturate at the high field values then the Langevin function is modified with an additional term $\chi_{\mathrm{a}} \mathrm{H}$ given by Eq. 5.2. This linear term presumably arises from final alignment of the any disordered spins such as the spins of the surface of the NP. $\chi_{\mathrm{a}}$ was determined from the linear component of the M vs. H variations at high field. The magnitudes of $\chi_{\mathrm{a}}$ obtained are listed in Fig. 5.7 where we do not see much variation in $\chi_{\mathrm{a}}$ with $\mathrm{T}$ or $\mathrm{D}$ implying that the magnetization saturates at low fields for the sample 11.7, 15, 21 and $23 \mathrm{~nm}$ as seen in Fig. 5.5 (b, c, d, e), but does not saturate for the $3.8 \mathrm{~nm}$ particle size and decreases with increase in $\mathrm{T}$ in Fig. 5.5a. This is likely from the larger percentages of disordered surface spins in the small $D=3.8 \mathrm{~nm}$ sample.

B. Variation of $\mathrm{M}_{\mathrm{o}}$ with $\mathrm{T}$

$M_{0}$ values have been extrapolated from the $M$ vs. $1 / \mathrm{H}$ plots for different temperatures above $T_{B}$. That is why the variations show a decrease in the temperature range investigated for larger particles in Fig. 5.7. 
C. Variation of $\mathrm{M}_{\mathrm{o}}$ with $\mathrm{D}$

The data in Fig. 5.7 show that $\mathrm{M}_{\mathrm{o}}$ increases with increase in the size of the NPs. For example, at $200 \mathrm{~K}, \mathrm{M}_{\mathrm{o}}$ increases from about $15 \mathrm{emu} / \mathrm{g}$ for $\mathrm{D}=3.8 \mathrm{~nm}$ to $\mathrm{M}_{\mathrm{o}}=26 \mathrm{emu} / \mathrm{g}$ for $\mathrm{D}=23 \mathrm{~nm}$. For bulk $\mathrm{Ni}, \mathrm{M}_{\mathrm{o}}=55 \mathrm{emu} / \mathrm{g}$. These results suggest that the lower value of $\mathrm{M}_{\mathrm{o}}$ with decrease in the size of the NPs is most likely due to disorder of the surface spins. A similar effect has been recently reported in $\mathrm{Fe}_{3} \mathrm{O}_{4}$ NPs [Pal et al, 2007] which follows the relation:

$$
M_{o}(D)=M_{o}(\text { bulk })\left[1-\frac{2 d}{D}\right]^{3}
$$

Where $M_{o}(D)$ is the saturation magnetization of particle size D with $d$ as the thickness of the disordered layer and $\mathrm{M}_{\mathrm{o}}$ (bulk) is the saturation magnetization for bulk.

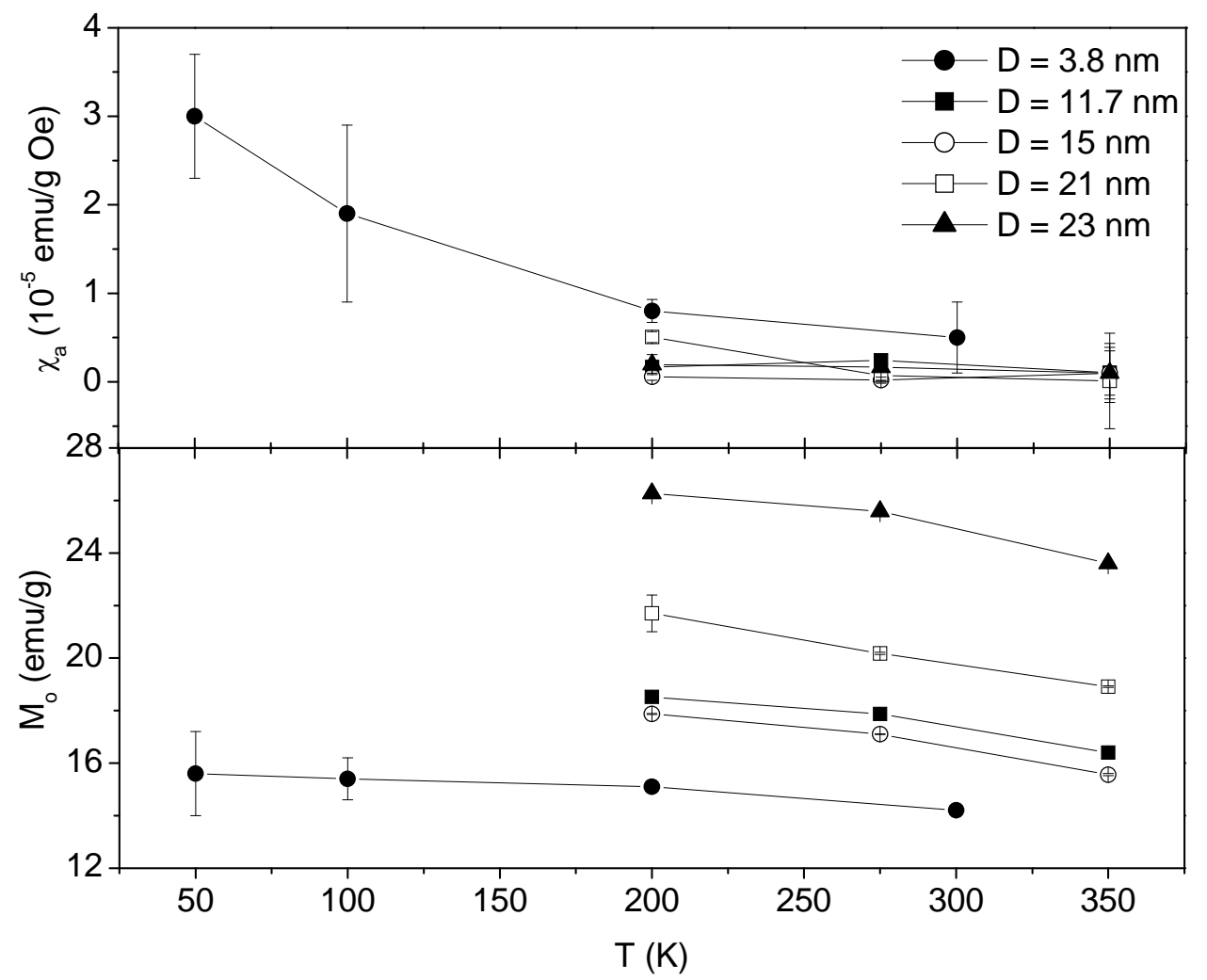

Fig. $5.7 \chi_{\mathrm{a}}$ and $\mathrm{M}_{\mathrm{o}}$ values used to obtain the collapsed curve above the blocking temperature. 


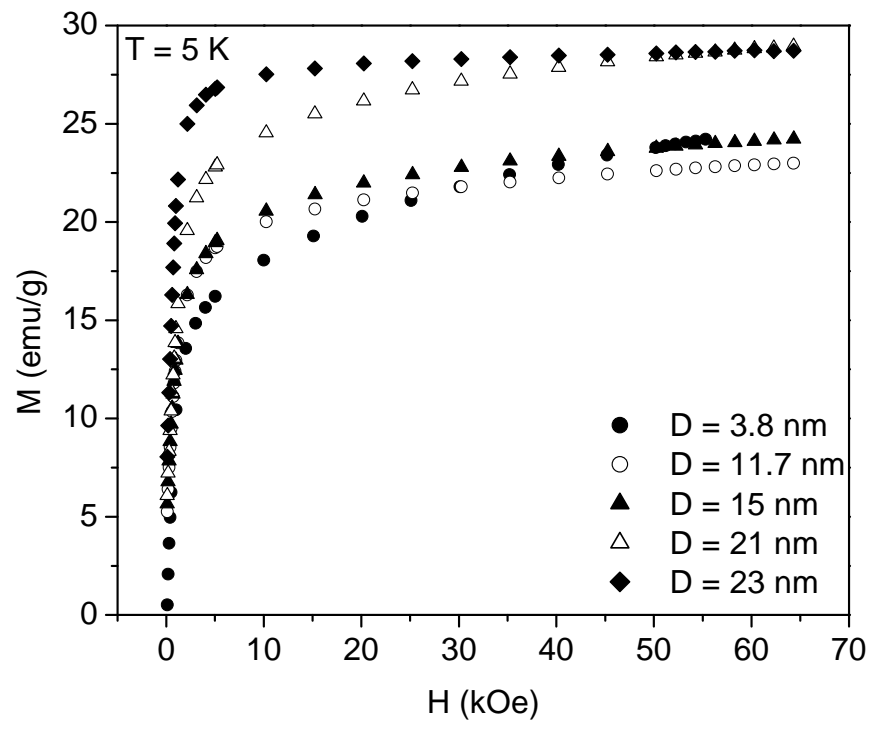

(a)

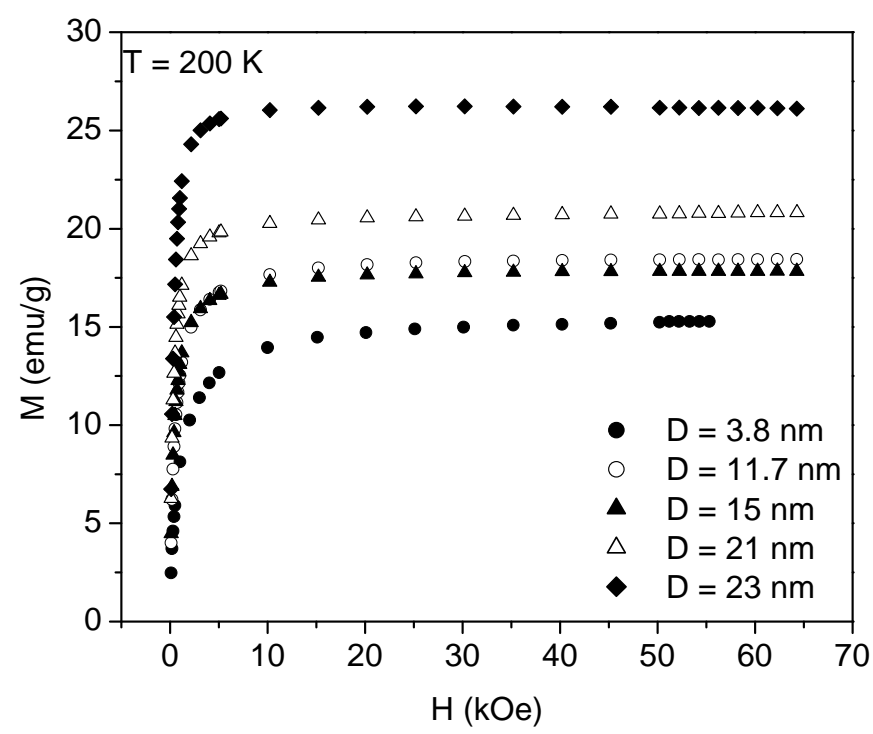

(b)

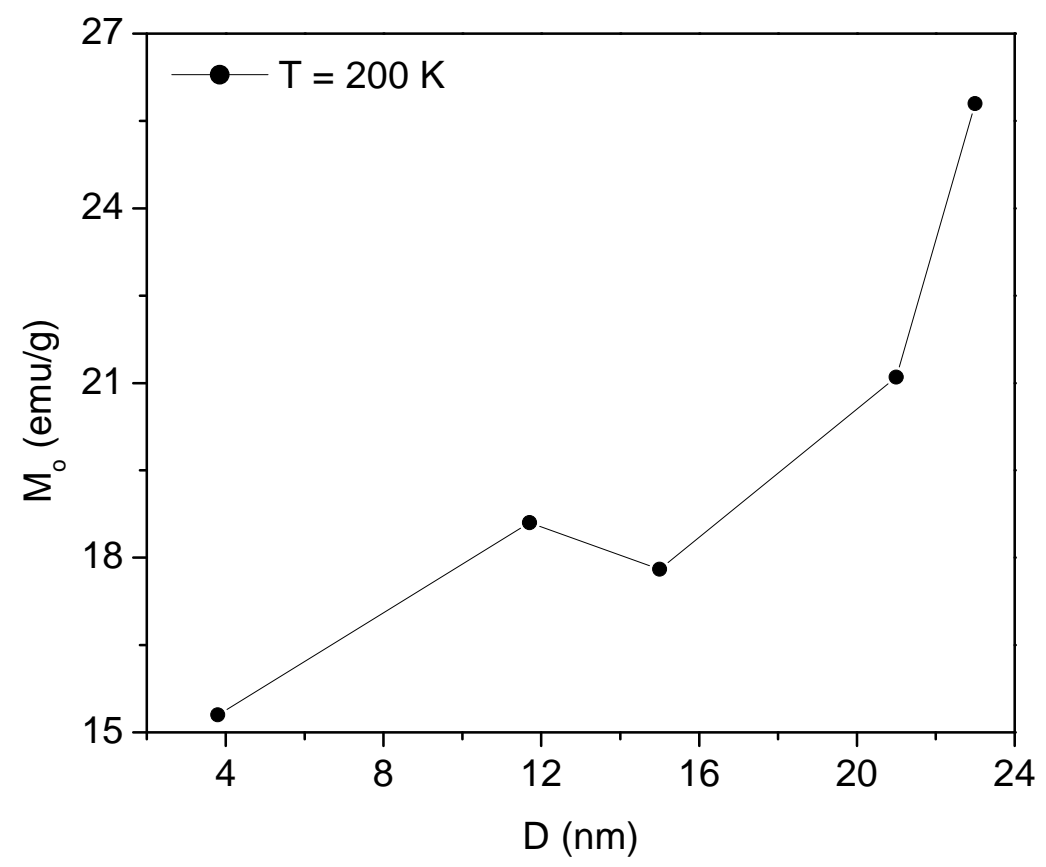

(c)

Fig. $5.8 \mathrm{M}$ vs. $\mathrm{H}$ plots at $\mathrm{T}=5,200 \mathrm{~K}$ with $\mathrm{M}_{\mathrm{o}}$ plotted for $\mathrm{T}=200 \mathrm{~K}$ extrapolated from high field for different $\mathrm{D}$. 


\subsection{Variation of Coercivity $\left(\mathrm{H}_{\mathrm{C}}\right)$ with Temperature $(\mathrm{T})$}

Another test to determine whether these particles are superparamagnetic involves the measurement of coercivity $\mathrm{H}_{\mathrm{C}}$ as a function of temperature which should go to zero above the blocking temperature [Zeng et al, 2002]]. Hysteresis measurements were done for the $15 \% \mathrm{Ni} / \mathrm{SiO}_{2}$ at various temperatures starting at $\mathrm{T}=2$ to $52 \mathrm{~K}$ to determine this effect. The coercivity decreases tending towards zero as the blocking temperature is approached from below. The data is fit to the theoretical equation given by [Fonseca, 2002]:

$$
H_{C}(T)=H_{C O}\left[1-\left(\frac{T}{T_{B}}\right)^{\frac{1}{2}}\right]
$$

When $\mathrm{T} \rightarrow \mathrm{T}_{\mathrm{B}} \mathrm{H}_{C} \rightarrow 0$. This variation for the $\mathrm{D}=3.8 \mathrm{~nm}$ NP is shown in Fig. 5.9. The above equation does not fit very well with the data below $7 \mathrm{~K}$ and deviates away from the data greater than $10 \mathrm{~K}$. This deviation for $\mathrm{T}>\mathrm{T}_{\mathrm{B}}$ from Eq. 5.12 may in part be due to the particle size distribution of one sample. Inset shows the variation of the remnance $\mathrm{M}_{\mathrm{r}}$.

$\mathrm{M}$ vs. $\mathrm{H}$ measurements were also done for the samples of size 11.7, 15, 21 and $23 \mathrm{~nm}$ at temperatures $\mathrm{T}=5$ and $200 \mathrm{~K}$ as shown in Fig. 5.10 (b, c, d, e) above $\mathrm{T}_{\mathrm{B}}$. In all these samples $\mathrm{H}_{\mathrm{C}}$ is not zero above the average $\mathrm{T}_{\mathrm{B}}$. It is most likely due to the size distribution of the particles so that the larger particles are still unblocked. The summarized results for coercivity are plotted in Fig. 5.11 for T=5 and $200 \mathrm{~K}$. 


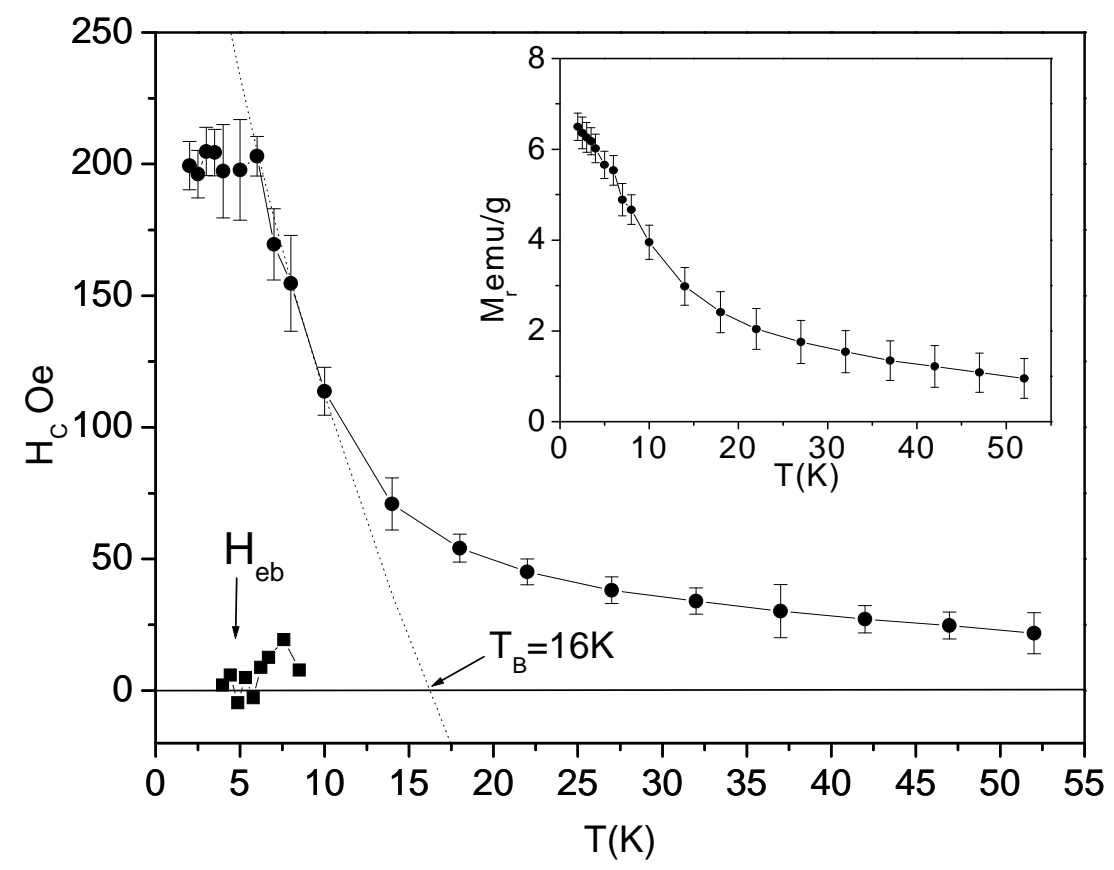

Fig. 5.9 Fitting of the measured coercivity against temperature for the $\mathrm{D}=3.8 \mathrm{~nm}$ sample. The line is a fit to Eq. 5.12 for $T_{B}=16 \mathrm{~K}$. The solid curve through the point is for visual aid. $\mathrm{H}_{\mathrm{eb}}$ is exchange bias measured for the sample cooled in $\mathrm{H}=20 \mathrm{kOe}$ to the measuring temperature.

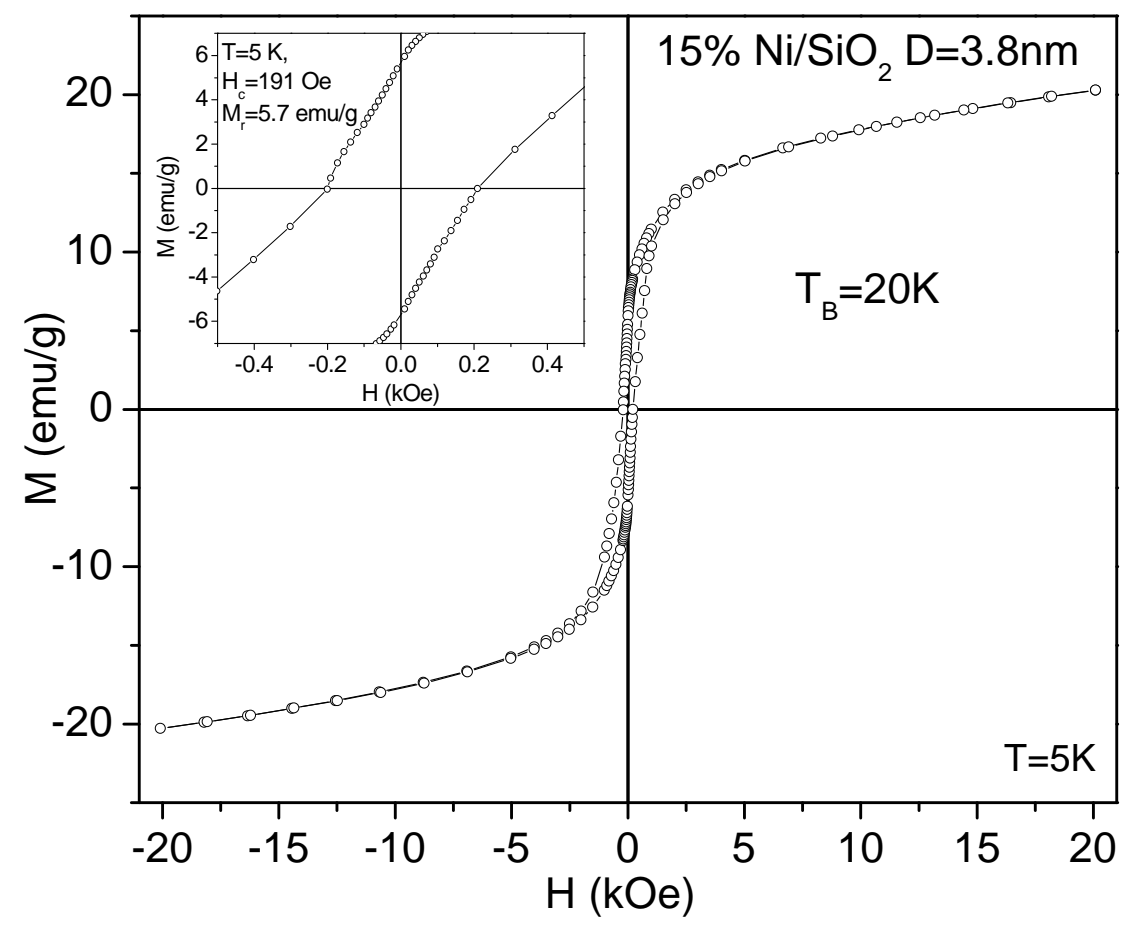

(a) 


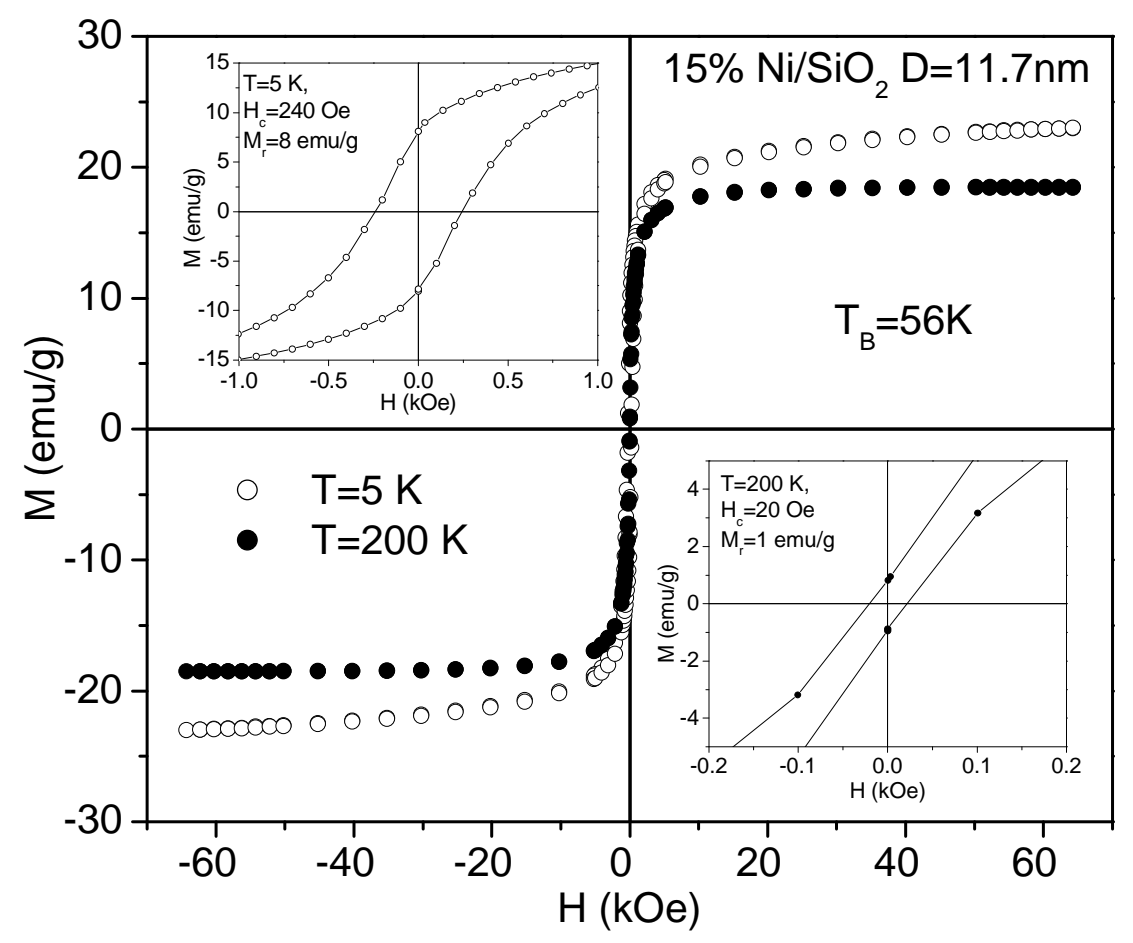

(b)

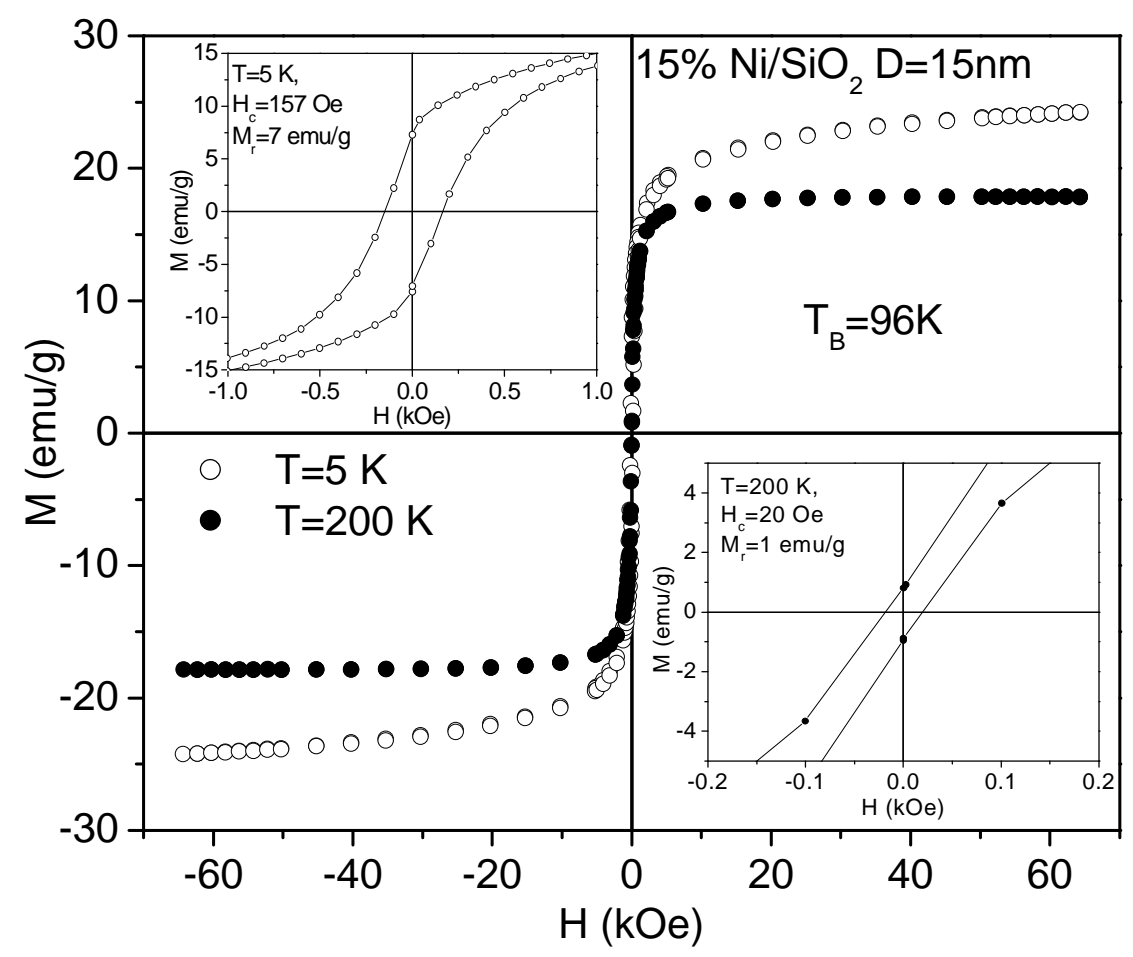

(c) 


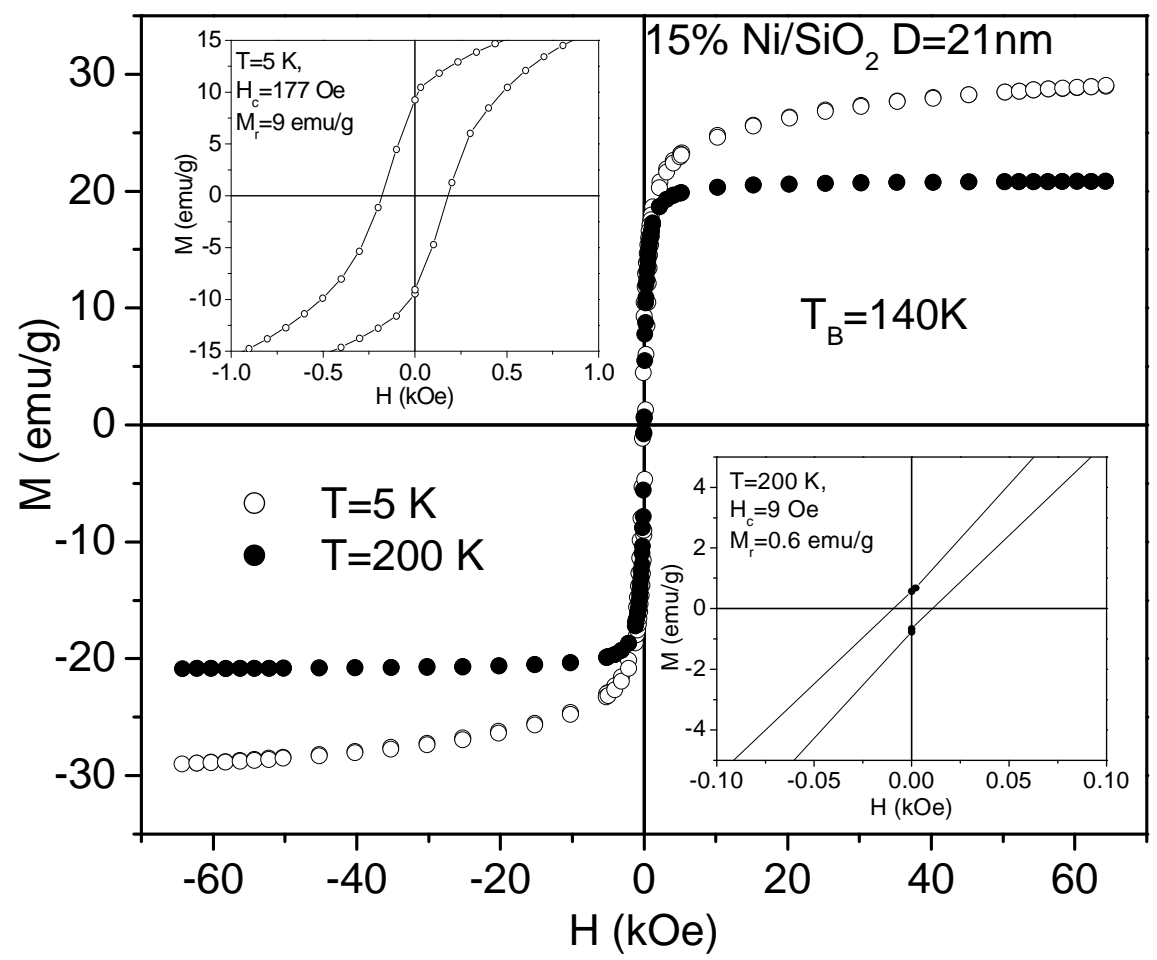

(d)

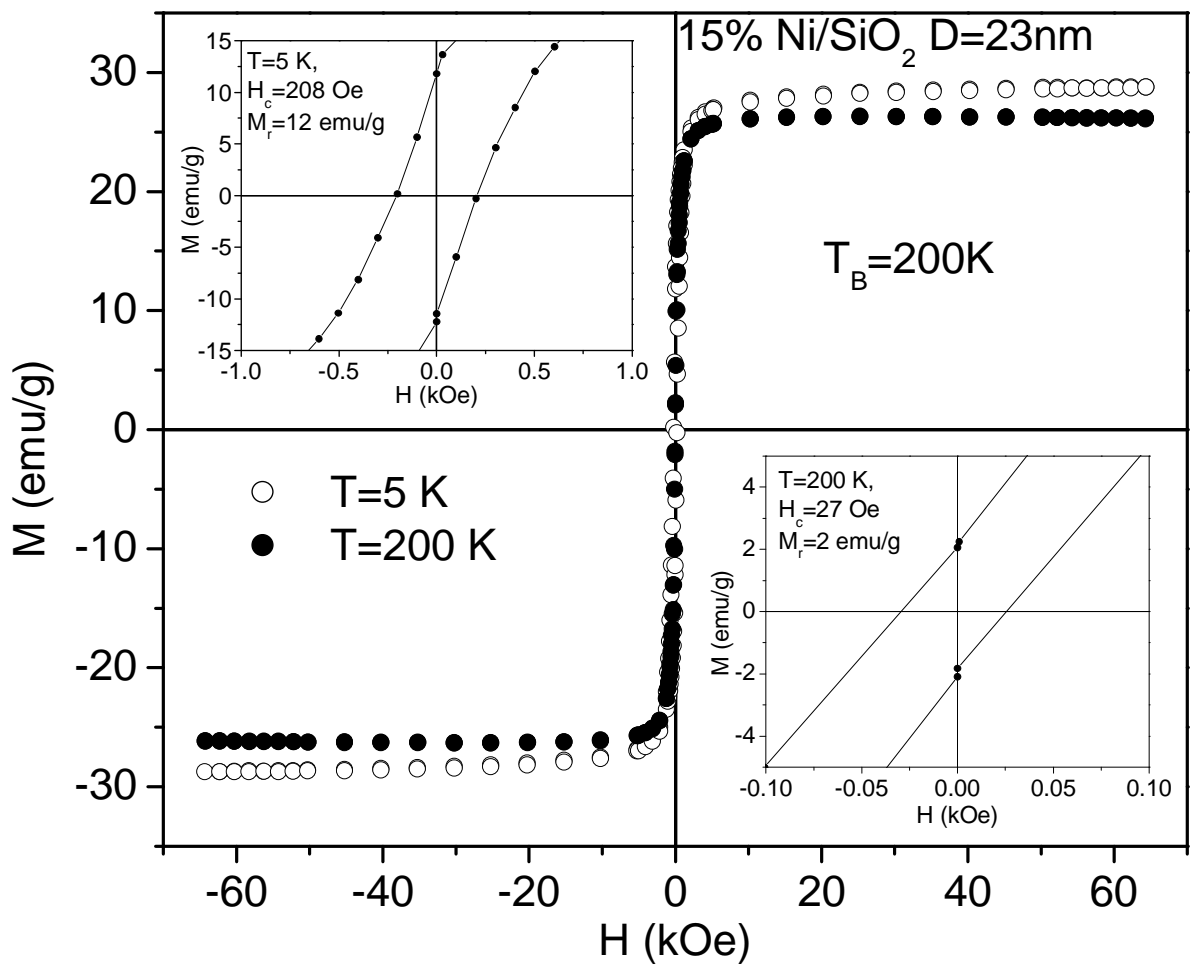

(e)

Fig. $5.10 \mathrm{M}$ vs. $\mathrm{H}$ plot at $\mathrm{T}=5$ and $200 \mathrm{~K}$ for different particle sizes. 


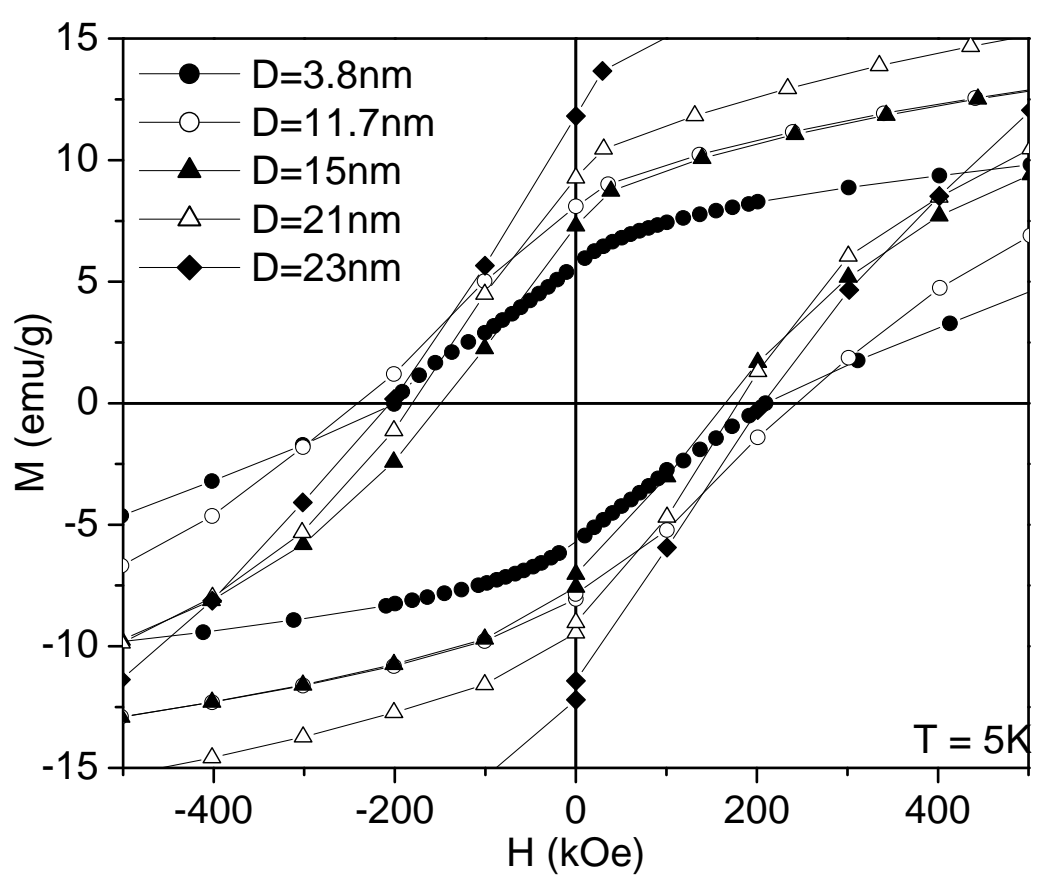

(a)

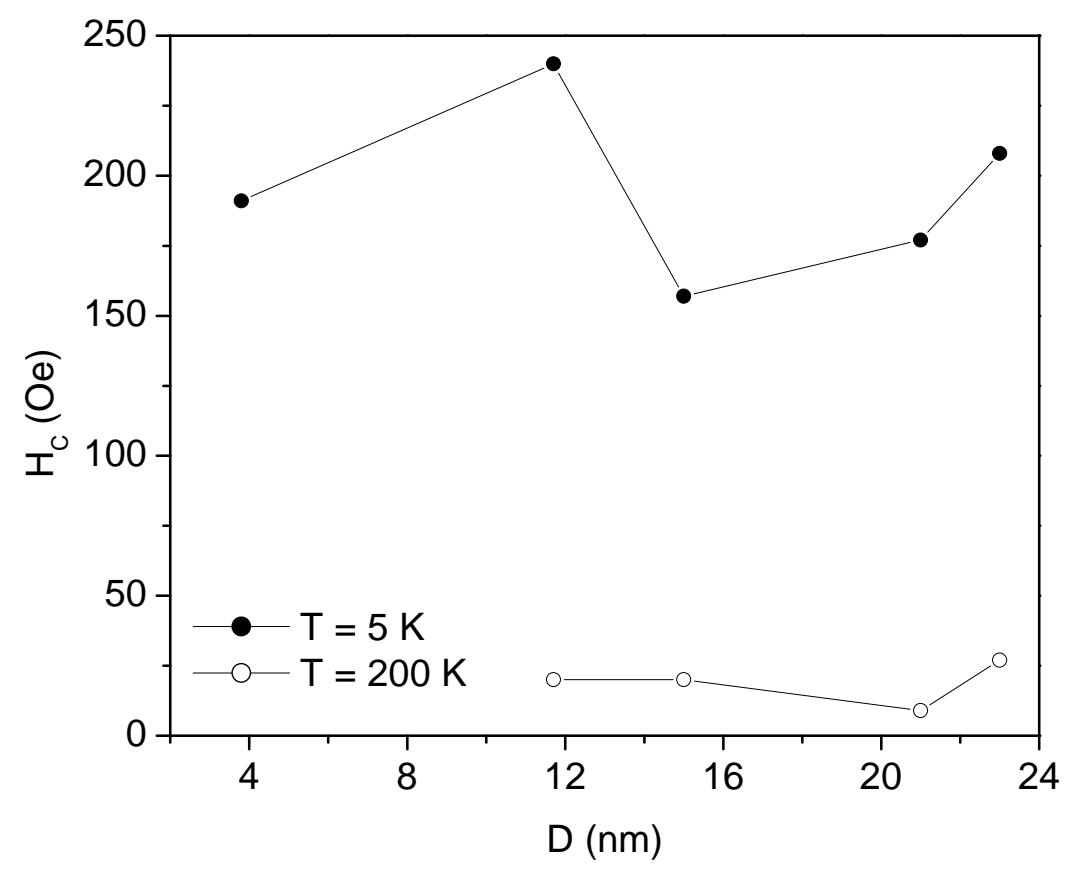

(b)

Fig. 5.11 Variation in $\mathrm{H}_{\mathrm{C}}$ with $\mathrm{D}$. 


\subsection{Exchange Bias $\left(\mathrm{H}_{\mathrm{eb}}\right)$}

For a ferromagnet, the hysteresis loop of $\mathrm{M}$ vs. $\mathrm{H}$ is symmetric about $\mathrm{H}=0$. In 1957, Meiklejohn and Bean while measuring the hysteresis loop of ferromagnetic Co particles which have been exposed to air during synthesis, reported a shifted hysteresis to the negative $\mathrm{H}$. This shift of the center of the loop from $\mathrm{H}=0$ is called exchange bias [e.g. see review by Nogués and Schuller, 1999]. It is now accepted that this loop shift occurs via exchange coupling across the interface between a ferromagnet (FM) and an antiferromagnet (AFM). For the $\mathrm{Co} / \mathrm{CoO}$ case, $\mathrm{Co}$ is a ferromagnet with $\mathrm{T}_{\mathrm{C}}=1338 \mathrm{~K}$ and $\mathrm{CoO}$ is an antiferromagnet with $\mathrm{T}_{\mathrm{N}}=291 \mathrm{~K}$. To observe $\mathrm{H}_{\mathrm{eb}}$, the sample needs to be cooled in a static field from a temperature above $T_{N}$ but below $T_{C}$ to temperature $T<T_{N}$.

For the case of $\mathrm{Ni}, \mathrm{Ni}$ is a ferromagnet with $\mathrm{T}_{\mathrm{C}}=627 \mathrm{~K}$ and bulk $\mathrm{NiO}$ is an antiferromagnet with $\mathrm{T}_{\mathrm{N}}=525 \mathrm{~K}$. Although for nanoparticles of $\mathrm{Ni}$ and $\mathrm{NiO}, \mathrm{T}_{\mathrm{C}}$ and $\mathrm{T}_{\mathrm{N}}$ are expected to be lowered because of reduced dimensionality of the nanoparticles. We checked for $\mathrm{H}_{\mathrm{eb}}$ in the $3.8 \mathrm{~nm}$ NPs of Ni by cooling the sample from room temperature to the measuring temperature (e.g. $2 \mathrm{~K}$ ) in $\mathrm{H}=20 \mathrm{kOe}$. No loop shift could be observed (Fig. 5.13) since the loop remains symmetrical. Measured $\mathrm{H}_{\mathrm{eb}}$ shown in Fig. 5.9 is essentially zero. This absence of $\mathrm{H}_{\mathrm{eb}}$ confirms the absence of any $\mathrm{NiO}$ in our samples.

A qualitative explanation of $\mathrm{H}_{\mathrm{eb}}$ borrowed by literature [Nogués et al, 1999] is given in Fig. 5.12. In Fig. 5.12(i) with the application of a field the FM layer is aligned along the direction of the field for $\mathrm{T}<\mathrm{T}_{\mathrm{C}}$ and the AFM layer is randomly oriented from $\mathrm{T}>\mathrm{T}_{\mathrm{N}}$. Upon cooling the sample in the presence of the field below $\mathrm{T}<\mathrm{T}_{\mathrm{N}}$ AFM layer gets ordered with the FM/AFM interface aligned ferromagnetically assuming FM interaction at the interface as shown in Fig. 5.12(ii). When the field is reversed the FM spins start to rotate but the spins at the interface of the AFM layer try to keep them ferromagnetically aligned by exerting a microscopic torque as it can be seen in Fig. 5.12(iii). Due to this effect the field required to rotate the FM spins is larger when it is in contact with the AFM layer until it is large enough to rotate all the spins in the FM layer Fig. 5.12(iv). Finally a smaller field is now required to rotate the FM spins back to their original position because the interaction of the AFM spins exert a torque in the same direction as the field Fig. 5.12(v). 


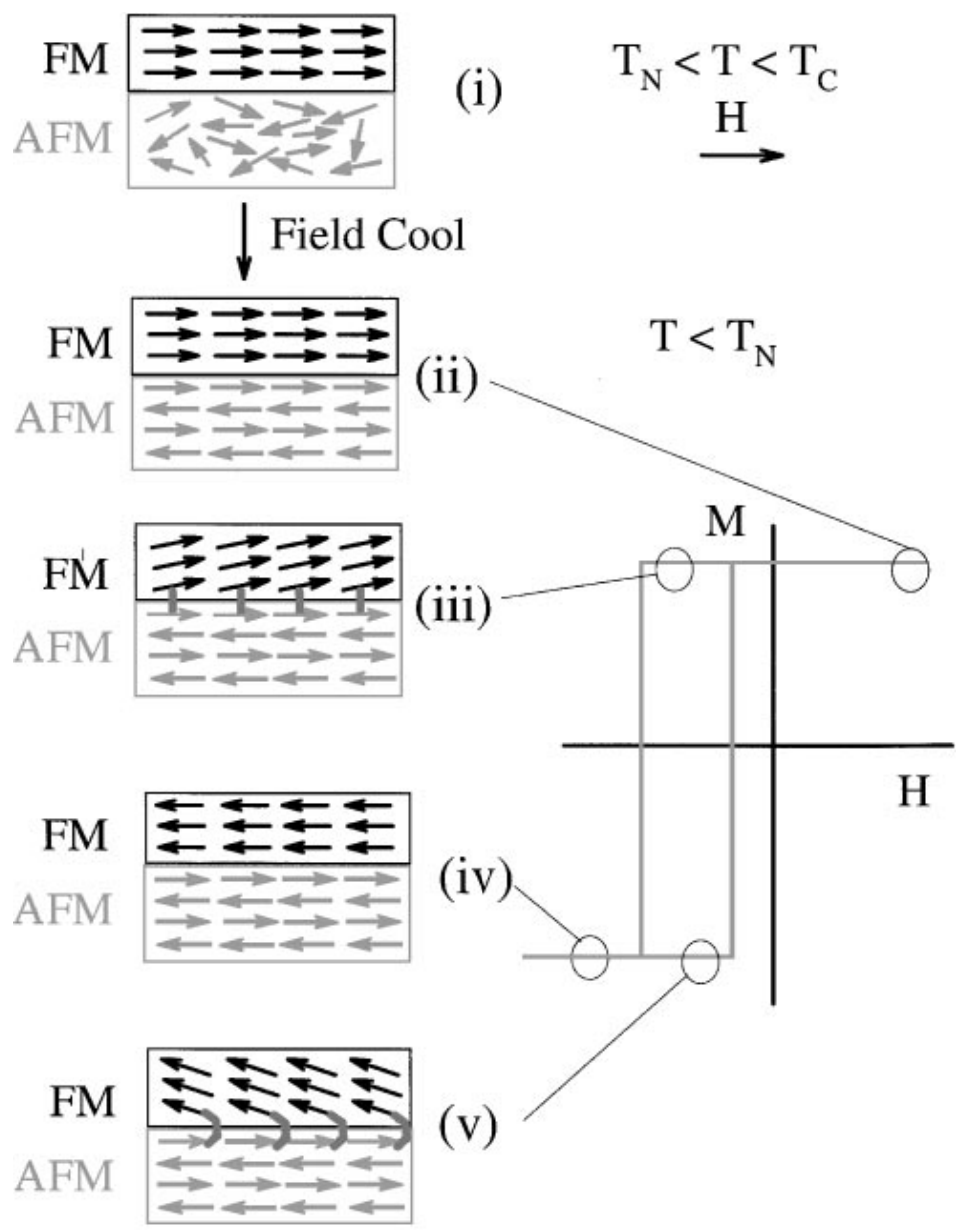

Fig. 5.12 Sample magnetization in a FM/AFM system in applied field from Nogués et al, 1999. 


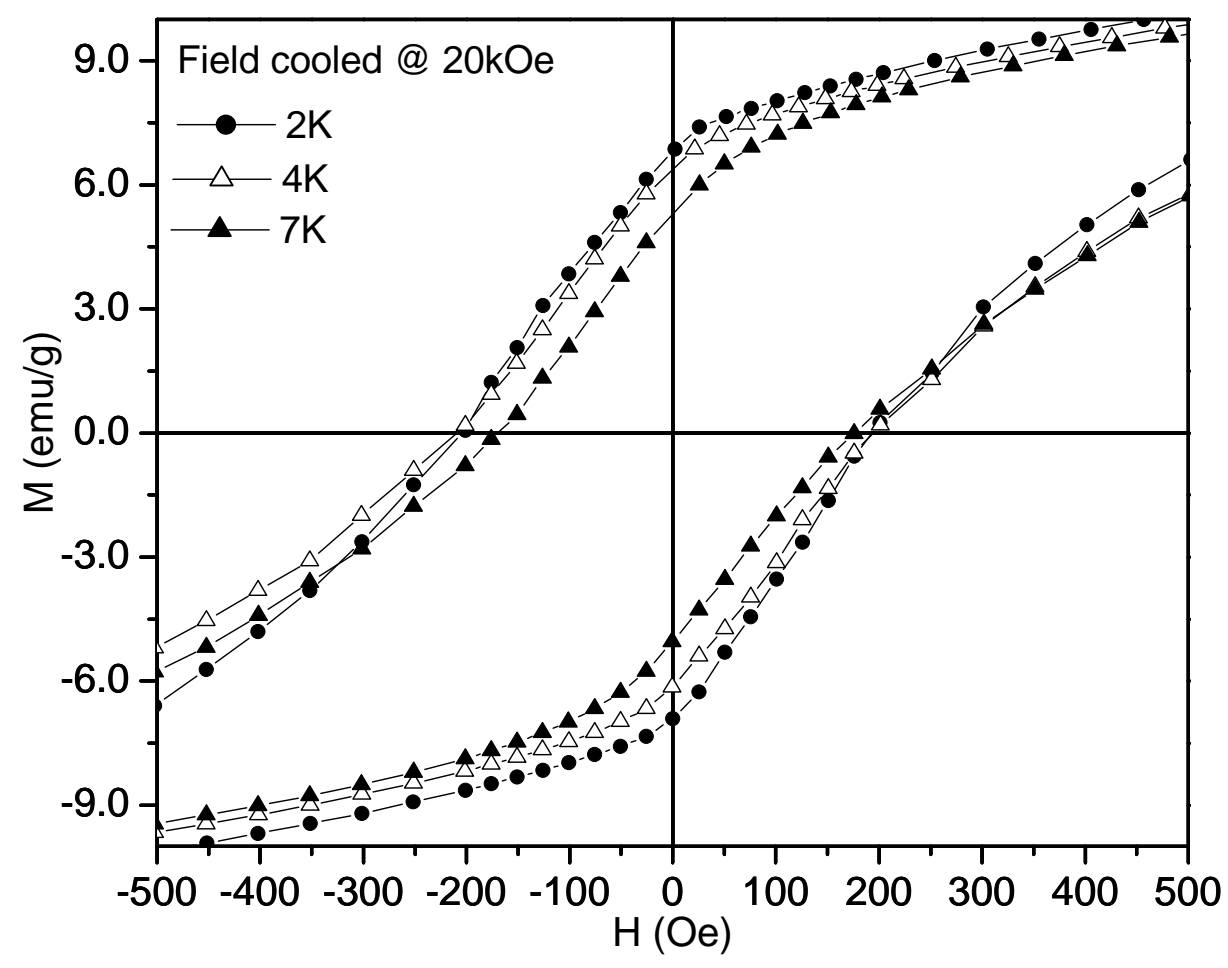

Fig. 5.13 Hysteresis loops measured at $\mathrm{T}=2,4,7 \mathrm{~K}$ for the $\mathrm{D}=3.8 \mathrm{~nm}$ sample cooled in $20 \mathrm{kOe}$. 


\subsection{AC Susceptibility}

The experimental data for AC susceptibility has been presented in the section below. This data has been evaluated based on the Néel-Arrhenius equation explained in the chapter 3. However, major results from chapter 3 are re-written below for convenience:

$$
T_{B}=\frac{T_{a}}{\ln \frac{f_{o}}{f_{m}}} \quad \text { where } \quad T_{a}=\frac{K_{a} V}{k}
$$

Equivalently the above equation can also be written as:

$$
\ln f_{m}=\ln f_{o}-\frac{T_{a}}{T_{B}}
$$

If there is a presence of interparticle interaction then Eq. 5.13 is modified to Eq. 5.15

$$
T_{B}=\frac{T_{a}}{\ln \left(\frac{f_{o}}{f_{m}}\right)}+T_{o}
$$

where $T_{o}$ represents the strength of the interparticle interaction.

Fig. 5.14 (a, b, c, d, e) for the different particle sizes show the AC susceptibility data where the blocking temperatures have been determined from the peaks in the $\chi$ " graphs. Measurements of $\chi^{\prime}$ and $\chi^{\prime \prime}$ were done using a commercial SQUID magnetometer with the measuring $h_{o}=7$ Oe and at frequencies $\mathrm{f}_{\mathrm{m}}=0.1,1,99,499$ and $997 \mathrm{~Hz}$. These measured value of $\chi^{\prime}$ and $\chi^{\prime \prime}$ were normalized to $15 \%$ concentration of $\mathrm{Ni}$ in the $\mathrm{Ni} / \mathrm{SiO}_{2}$ $(15 / 85)$ samples.

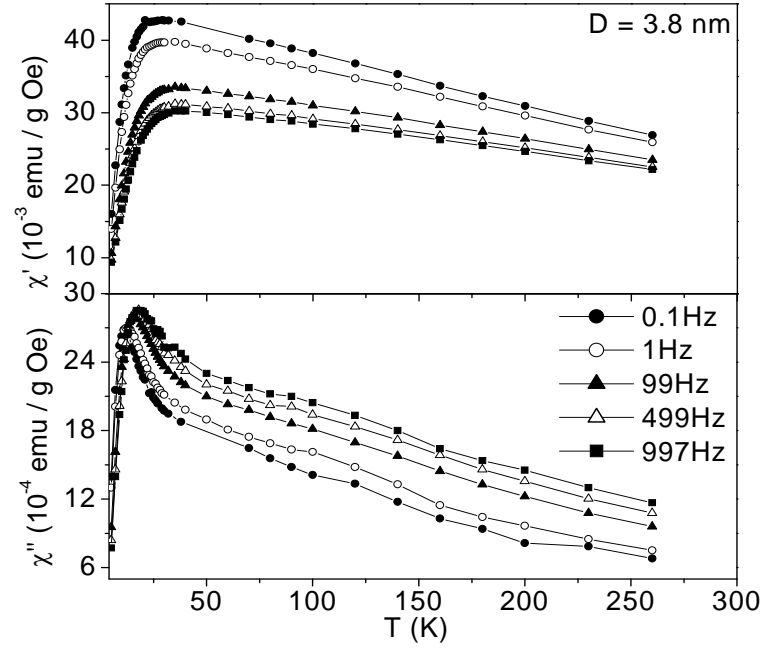

(a)

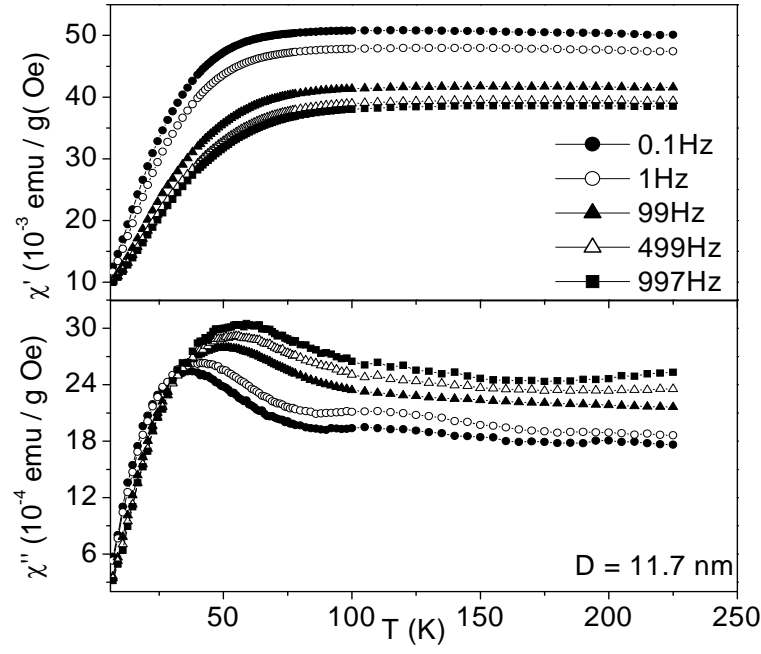

(b) 


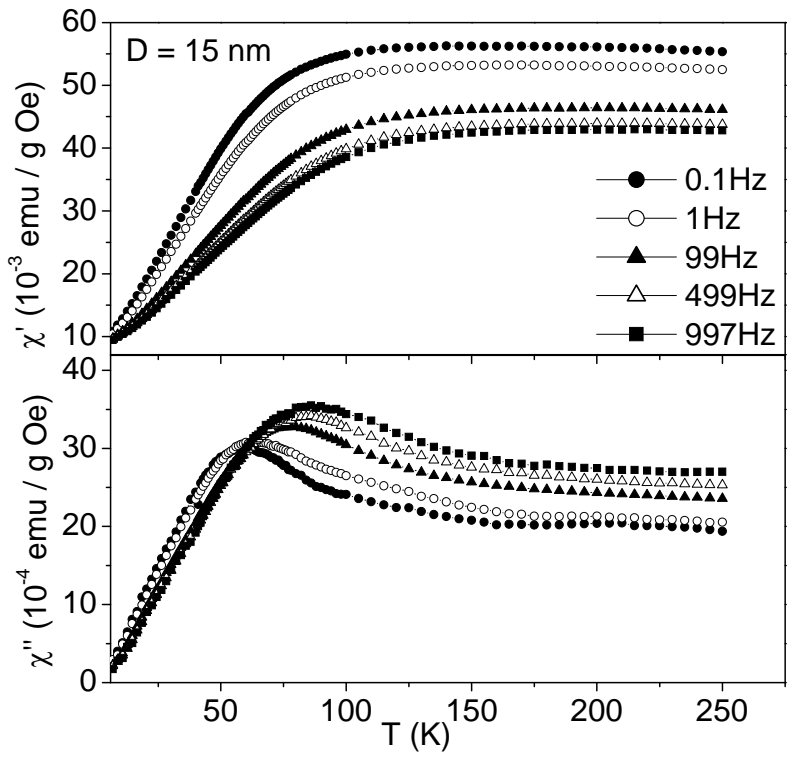

(c)

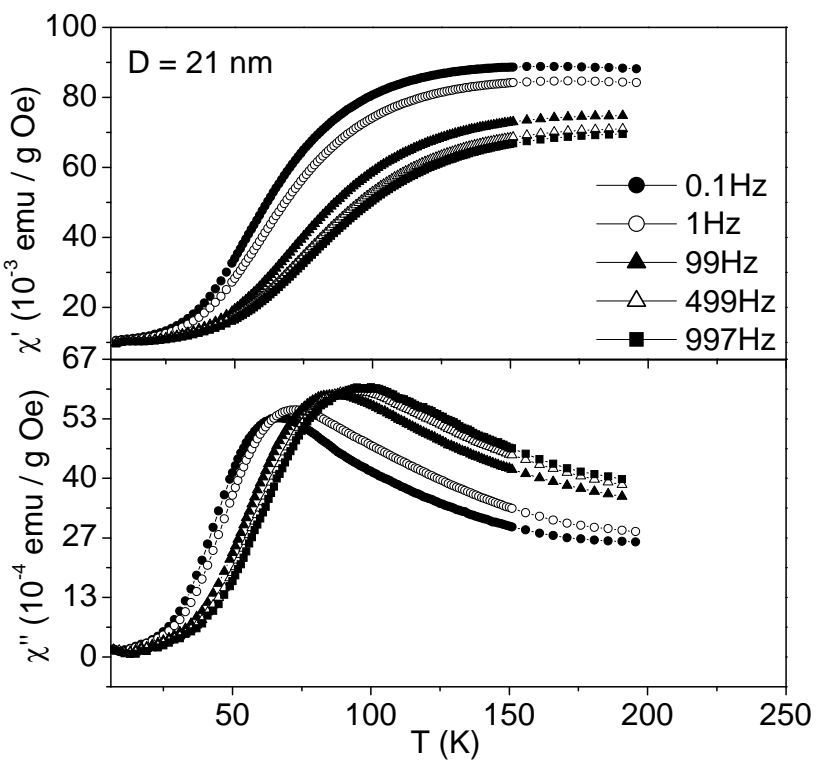

(d)

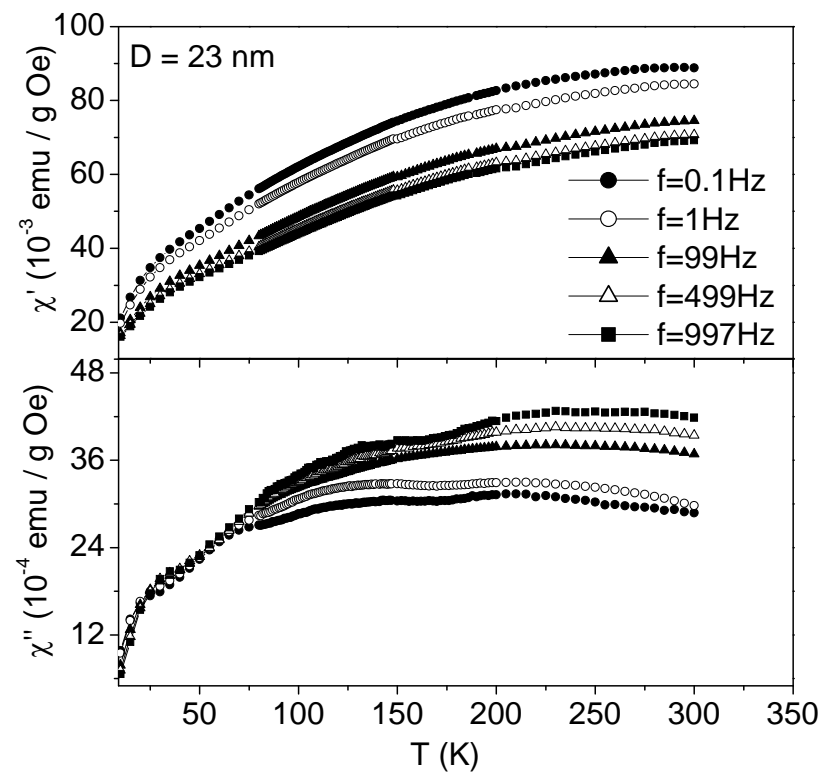

(e)

Fig. 5.14 Temperature variations of the AC susceptibilities $\chi^{\prime}$ and $\chi^{\prime \prime}$ for different measuring frequencies, for the five samples investigated in this work. 
It is evident that $T_{B}$ determined by the peak in $\chi^{\prime \prime}$ increases with increase in $f_{m}$ as predicted by Eqs. 5.13 and 5.15. Using the measured values of $T_{B}$ for each $f_{m}$, we plot $\operatorname{lnf}_{\mathrm{m}}$ vs. $1 / \mathrm{T}_{\mathrm{B}}$ following Eq. 5.14. The data fits the predicted linear behavior with the intercept yielding $\operatorname{lnf}_{\mathrm{o}}$ and the slope yielding $\mathrm{T}_{\mathrm{a}}$. Using least-squares fitting of the data shown in Fig. 5.15, magnitudes of $f_{o}$ are determined to be $2.83\left(\begin{array}{c}+1.2 .6 \\ -1.2\end{array}\right) \times 10^{9} \mathrm{~Hz}$ for the $\mathrm{D}=3.8 \mathrm{~nm}$ sample and $\mathrm{f}_{\mathrm{o}}=1.82\left(\begin{array}{c}+0.94 \\ -0.62\end{array}\right) \times 10^{10} \mathrm{~Hz}, \mathrm{f}_{\mathrm{o}}=1.72\left(\begin{array}{c}+0.25 \\ -0.23\end{array}\right) \times 10^{11} \mathrm{~Hz}$ and $\mathrm{f}_{\mathrm{o}}=3.96\left(\begin{array}{c}+4.62 \\ -2.13\end{array}\right) \times$ $10^{11} \mathrm{~Hz}$ for the $\mathrm{D}=11.7,15$ and $21 \mathrm{~nm}$ samples respectively.

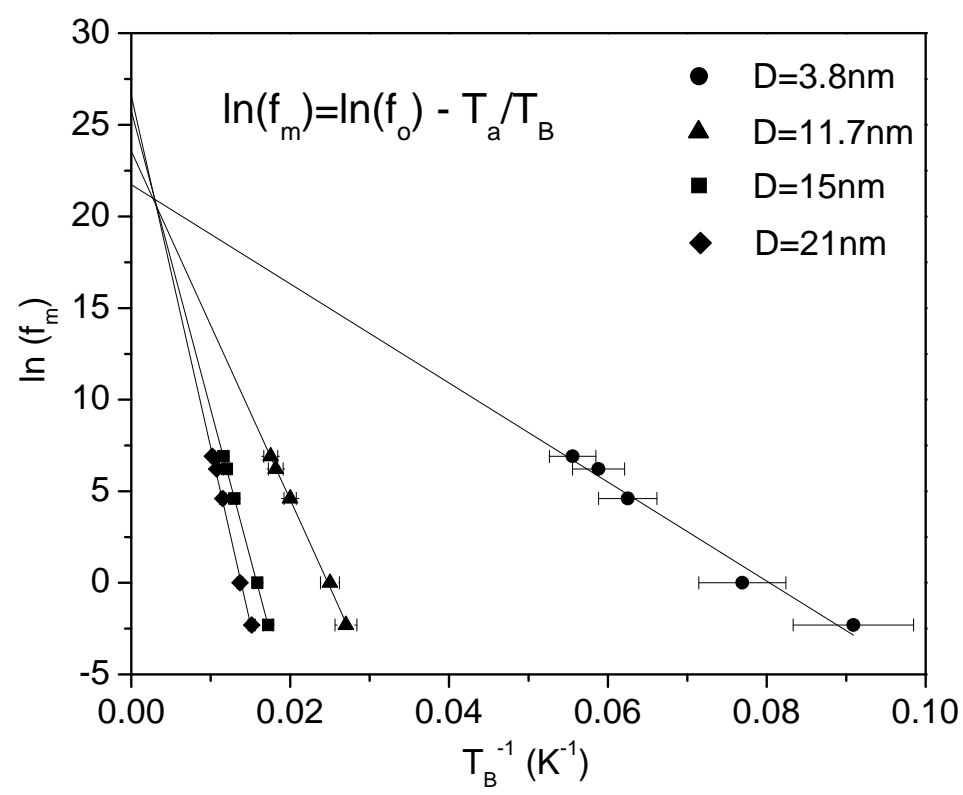

Fig. 5.15 Plots of $\operatorname{lnf}_{m}$ vs. $T_{B}{ }^{-1}$, the solid line is a least-square fit to yield $f_{0}$.

For the two larger particles, the magnitude of $f_{0}$ is higher by an order of magnitude. It is known that the presence of IPI often leads to increased magnitudes of $f_{o}$ [Shim et al, 2006] when data are fit to Eq. 5.13. Taking into consideration the relatively large uncertainty in $f_{0}$ for the smallest $D=3.8 \mathrm{~nm} \mathrm{NPs}$, we have chosen $\mathrm{f}_{0}=1.82 \times 10^{10} \mathrm{~Hz}$ for the $\mathrm{D}=11.7 \mathrm{~nm}$ NPs as the likely magnitude for $\mathrm{f}_{\mathrm{o}}$ for this system. Using the magnitude of $\mathrm{f}_{\mathrm{o}}=1.82 \times 10^{10} \mathrm{~Hz}$ the data are plotted as $\mathrm{T}_{\mathrm{B}}$ against $1 / \ln \left(\mathrm{f}_{\mathrm{o}} / \mathrm{f}_{\mathrm{m}}\right)$ in Fig. 5.16 to check the validity of Eq. 5.15. The least-squares fits are then used to determine $T_{a}$ and $T_{o}$ as $\mathrm{T}_{\mathrm{a}}(\mathrm{K})=310(21), 954(17), 1334(14)$ and 1405(47) for $\mathrm{D}=3.8,11.7,15$ and $21 \mathrm{~nm}$ respectively along with $\mathrm{T}_{\mathrm{o}}$ (representing the inter-particle interaction) $=0,0,6.6(0.7)$ and $12.5(2.5) \mathrm{K}$. 


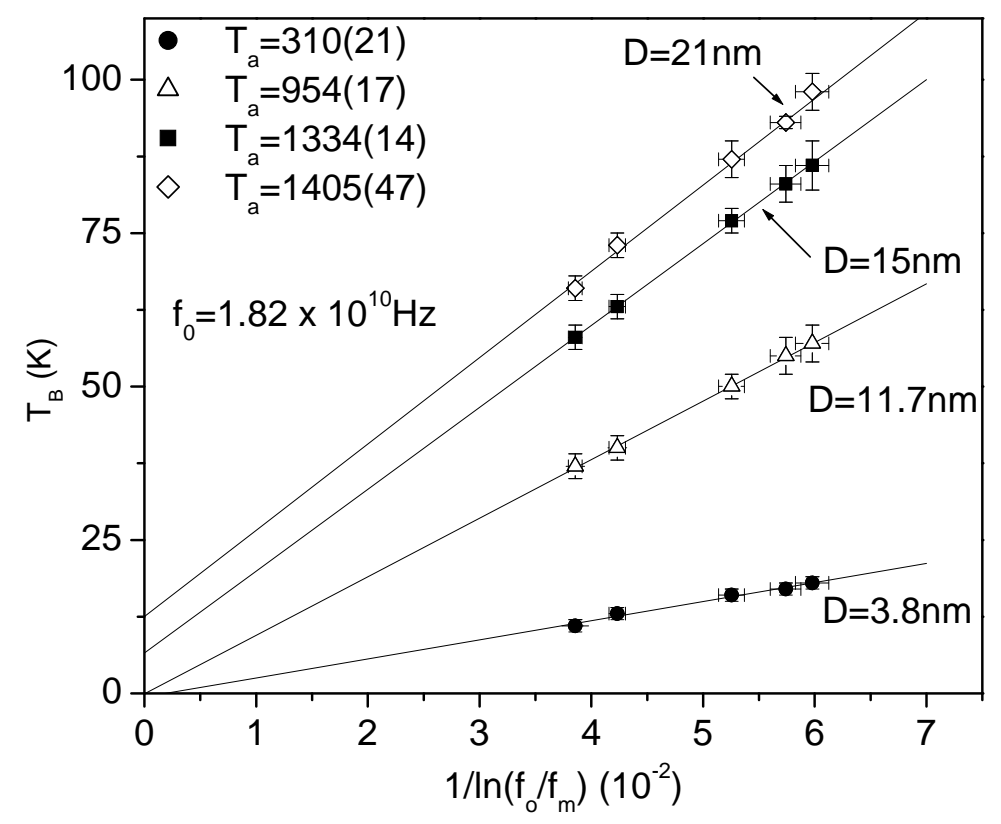

Fig. 5.16 Plots of $T_{B}$ against $1 / \ln \left(f_{o} / f_{m}\right)$, the solid line is a least-square fit to yield $\mathrm{T}_{\mathrm{o}}$ and $\mathrm{T}_{\mathrm{a}}$.

For the two larger particles, the magnitude of $f_{o}$ is higher by an order of magnitude since the presence of IPI often leads to increased magnitudes of $f_{o}$ and $T_{a}$ with increase in the size D of the Ni NPs. For $\mathrm{f}_{0}$, the largest estimated uncertainty is for the $\mathrm{D}=3.8 \mathrm{~nm}$ yielding $\mathrm{f}_{\mathrm{o}}=2.8\left(\begin{array}{c}+11.6 \\ -2.2\end{array}\right) \times 10^{9} \mathrm{~Hz}$. In an earlier publication, we reported $\mathrm{f}_{\mathrm{o}}=2.6 \times 10^{9} \mathrm{~Hz}$ for this sample using an eye-ball fit of the data [Singh et al, 2008] similar to the value of $\mathrm{f}_{\mathrm{o}}=1.8 \times 10^{9} \mathrm{~Hz}$ reported by [Goya et al, 2003] in a $5 \% \mathrm{Ni} / \mathrm{SiO}_{2}$ sample. This value is consistent with $\mathrm{f}_{\mathrm{o}}=1.82 \times 10^{10} \mathrm{~Hz}$ used here since for $\mathrm{f}_{\mathrm{o}}=2.8\left(\begin{array}{c}+11.6 \\ -2.2\end{array}\right) \times 10^{9} \mathrm{~Hz}$ determined above using least-squares fitting has large enough uncertainty to accommodate $\mathrm{f}_{\mathrm{o}}=1.82 \times 10^{10} \mathrm{~Hz}$. The important result of this analysis is that using a size independent $f_{o}$, the presence of measurable IPI is evident in the two larger sizes and $T_{a}$ increases with increase in the size of D (see Fig. 5.17) of the NPs as qualitatively expected. Later we show that the magnitude of $\mathrm{f}_{\mathrm{o}}=1.8 \times 10^{10} \mathrm{~Hz}$ determined above is consistent with calculations also using a theoretical expression. 


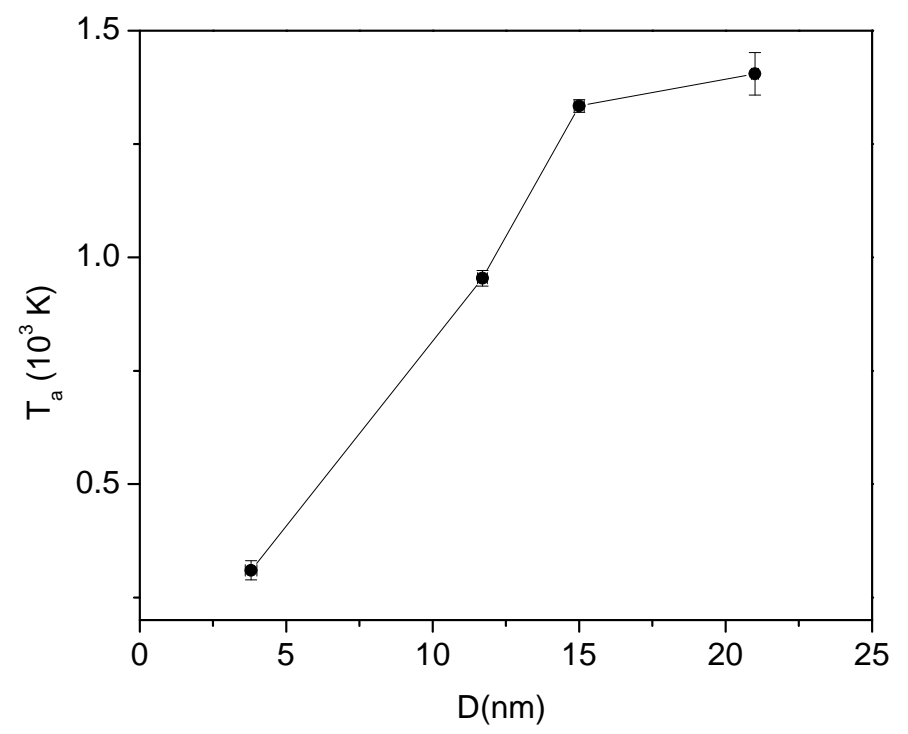

Fig. 5.17 Plot of $T_{a}$ vs. D.

Next, the magnitudes of $T_{a}=K_{a} V / k$ are used to determine $K_{a}$ representing the energy barrier assuming spherical NPs. For the cubic anisotropy of $\mathrm{Ni}, \mathrm{K}_{\mathrm{a}}=\mathrm{K}_{1} / 12$ where $\mathrm{K}_{1}$ is the first-order anisotropy constant with $\mathrm{K}_{1}=|7.5| \times 10^{5} \mathrm{ergs} / \mathrm{cm}^{3}$ for bulk Ni [Gittleman et al, 1974]. The computed values of $\left|\mathrm{K}_{1}\right|$ (in units of $10^{5} \mathrm{ergs} / \mathrm{cm}^{3}$ ) are 178.7, $18.8,12.5$ and 4.8 respectively for the $\mathrm{D}=3.8,11.7,15$ and $21 \mathrm{~nm}$ samples. The observed increase in $\mathrm{K}_{1}$ with decreasing D (see Fig. 5.18) has been reported and discussed in other systems also, the source being increasing surface anisotropy with decreasing D [Bodkar et al, 1994, Gilmore et al, 2005, Yanes et al, 2007, Shim et al, 2008]. For the largest $\mathrm{D}=21 \mathrm{~nm}$ sample, the magnitude of $\mathrm{K}_{1}$ is close to the value for bulk Ni.

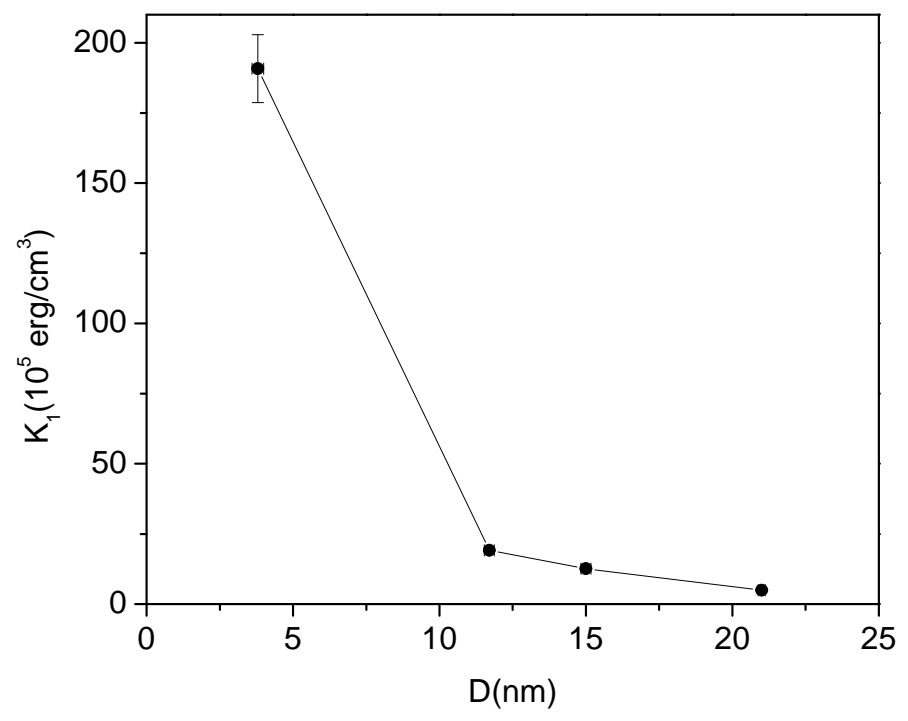

Fig. 5.18 Plot of $K_{1}$ vs. D. 


\subsection{Theoretical Estimation of Attempt Frequency $\left(f_{0}\right)$}

Next we calculate the theoretically expected magnitude of the attempt frequency $f_{0}$. Based on the earlier work by Brown [Brown, 1963], a simplified expression for $f_{o}$ in zero field given by Aharoni [Aharoni, 1973] for the case of $T_{a}>>T$ reduces to the following for the case of $\mathrm{Ni}$ :

$$
f_{o}=\frac{\gamma_{o} 2 K_{a}}{M_{S}} \sqrt{\frac{T_{a}}{\pi T}} \text { where } K_{a}=\frac{K_{1}}{12}
$$

Here $\gamma_{o}$ is the gyromagnetic ratio, and $\mathrm{M}_{\mathrm{s}}$ is the saturation magnetization. For Ni with $\mathrm{g}=2.2, \gamma_{\mathrm{o}}=2 \pi \mathrm{g} \mu_{\mathrm{B}} / \mathrm{h}=1.935 \times 10^{7}$. For $\mathrm{T}$ in Eq. 5.16, we use the average $\mathrm{T}_{\mathrm{B}}$ measured in the frequencies $\mathrm{f}_{\mathrm{m}}$ used in our experiments since $\mathrm{f}_{\mathrm{o}}$ was determined at these temperatures and the condition $T_{a}>>T$ is still valid using the $T_{a}$ values noted in Fig. 5.16. We used the values of $\mathrm{M}_{\mathrm{s}}=245,216,229$ and $274 \mathrm{emu} / \mathrm{cm}^{3}$ measured at $5 \mathrm{~K}$ for the $\mathrm{D}=3.8,11.7,15$ and $21 \mathrm{~nm}$ samples respectively. These magnitudes of $\mathrm{M}_{\mathrm{s}}$ are only about $50 \%$ of the corresponding magnitude for bulk Ni likely due to disorder of spins on the surface of a NP. For the $D=3.8 \mathrm{~nm}$ with $\mathrm{T}_{\mathrm{a}}=310 \mathrm{~K}$, Eq. 5.16 yields $\mathrm{f}_{\mathrm{o}}=6.0 \times 10^{11} \mathrm{~Hz}$. Similar calculations for the other samples yield the following $\mathrm{f}_{\mathrm{o}}$ values: $7.1 \times 10^{10} \mathrm{~Hz}$ for $11.7 \mathrm{~nm}$, $4.2 \times 10^{10} \mathrm{~Hz}$ for $15 \mathrm{~nm}$ and $1.3 \times 10^{10} \mathrm{~Hz}$ for $21 \mathrm{~nm}$. Except for the smallest NPs, these calculated magnitudes of $f_{o}$ using Eq. 5.16 compare quite favorably with $f_{o}=1.8 \times 10^{10} \mathrm{~Hz}$ used in the analysis.

In summary, the variations of the magnetic relaxation parameters $T_{B}$ and $T_{a}$ with size of Ni NPs dispersed in silica matrix are shown to follow the predictions of Eqs. 5.13 to 5.15 with a size independent $f_{o}$ and the presence of a weak IPI in the larger NPs. The order of magnitude of the calculated $f_{0}$ is in agreement with the measured $f_{0}$.

\subsection{Interparticle Interaction by evaluation of the parameter $\Phi$}

By the evaluation of the parameter $\Phi=\Delta \mathrm{T}_{\mathrm{B}} /\left[\mathrm{T}_{\mathrm{B}} \Delta \log _{10} \mathrm{f}_{\mathrm{m}}\right]$ which represents fractional change in $T_{B}$ per decade change in $f_{m}$ [Dormann et-al, 1988], one can determine the effect of interparticle interaction. Experiments have shown that $\Phi$ is very small (0.005-0.05) for spin glasses and $\Phi \geq 0.13$ for isolated non-interacting NPs 
[Dormann et al, 1988]. For intermediate values $(0.005<\Phi<0.13)$, interparticle interaction is present with its effect decreasing with increasing $\Phi$. Determining $\Delta \mathrm{T}_{\mathrm{B}}$ for maximum and minimum $\mathrm{f}_{\mathrm{m}}$ in our experiments, $\Phi=0.16,0.13,0.12$ and 0.12 is found respectively for the $\mathrm{D}=3.8,11.7,15$ and $21 \mathrm{~nm}$ samples. These magnitudes of $\Phi$ suggest the presence of a weak IPI in the larger $\mathrm{D}=15 \mathrm{~nm}$ and $\mathrm{D}=21 \mathrm{~nm}$ particles (possibly due to larger moment per particle) and absence of IPI in the two smaller particles. This is consistent with the analysis presented above.

\subsection{Theoretical Simulations of AC Susceptibility}

Simulations have been done based on equations derived earlier in chapter 4 where the equations for $\chi^{\prime}$ and $\chi^{\prime \prime}$ have been obtained. The simulations are based on those equations rewritten here for convenience:

$$
\begin{aligned}
& \chi^{\prime}=\frac{M_{S}^{2}}{3 K}\left[1+\frac{K_{a} V}{k_{B} T} \frac{1}{1+(\omega \tau)^{2}}\right] \\
& \chi^{\prime \prime}=\frac{M_{S}{ }^{2}}{3 K}\left[\frac{K_{a} V}{k_{B} T} \frac{\omega \tau}{1+(\omega \tau)^{2}}\right]
\end{aligned}
$$

The value of $\mu_{\mathrm{o}}$ is taken to be unity since the calculations have been done in CGS units for comparison. Values for $\mathrm{M}_{\mathrm{S}}$ have been obtained from the extrapolation of the high field region of the $\mathrm{M}$ vs. $\mathrm{H}$ plots at $\mathrm{T}=5 \mathrm{~K} . \mathrm{K}_{\mathrm{a}}$ has been determined from the energy barriers obtained from the plots in Fig. 5.16.

Theoretical simulations based on $\chi^{\prime}$ and $\chi^{\prime \prime}$ have been done on all the samples with $\mathrm{D}=3.8,11.7,15$ and $21 \mathrm{~nm}$ as shown in Fig 5.18 ( $\mathrm{a}, \mathrm{b}, \mathrm{c}, \mathrm{d})$. The table below the figures summarizes the experimental and simulated values of $\mathrm{T}_{\mathrm{B}}$. The simulated curves follow a similar behavior as the experimental data except for the fact that the experimental data yields broader peaks possibly arising from particle size distribution. The blocking temperature for the simulated curves are about 5\% higher also for the value of $f_{0}=1.82 \times 10^{10} \mathrm{~Hz}$ used here. Decreasing the value of $f_{o}$ increases the discrepancy even more whereas increasing $f_{0}$ brings it closer to the experimental values. 


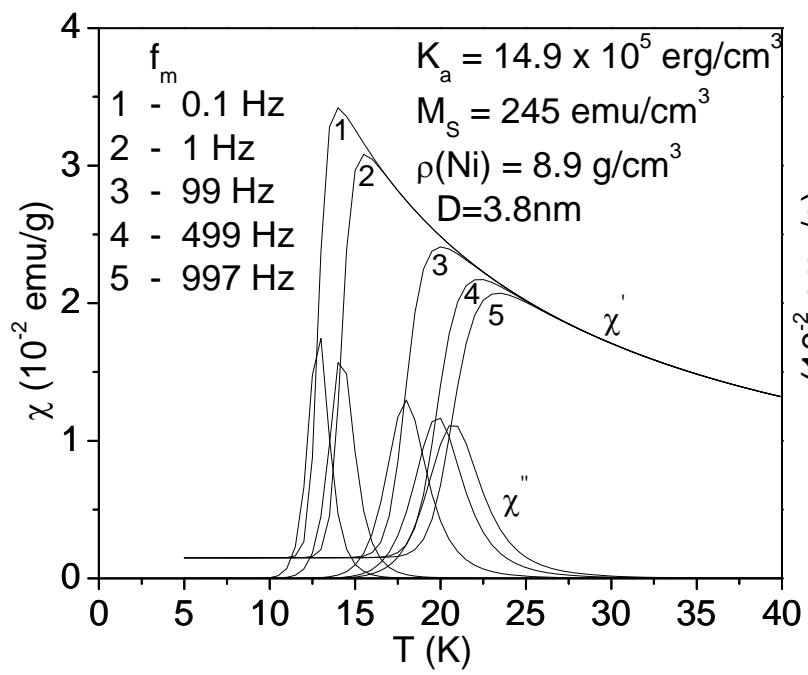

(a)

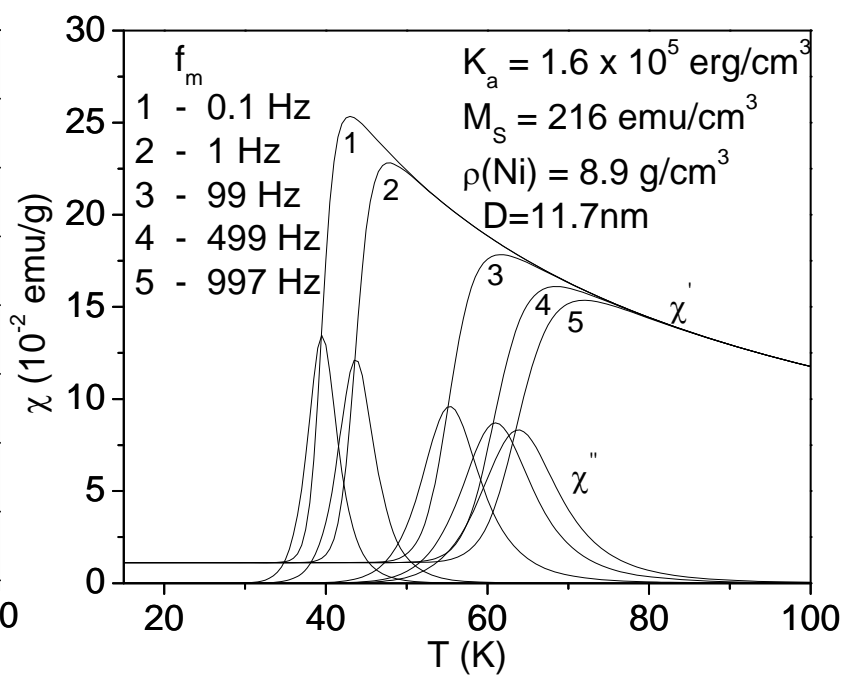

(b)

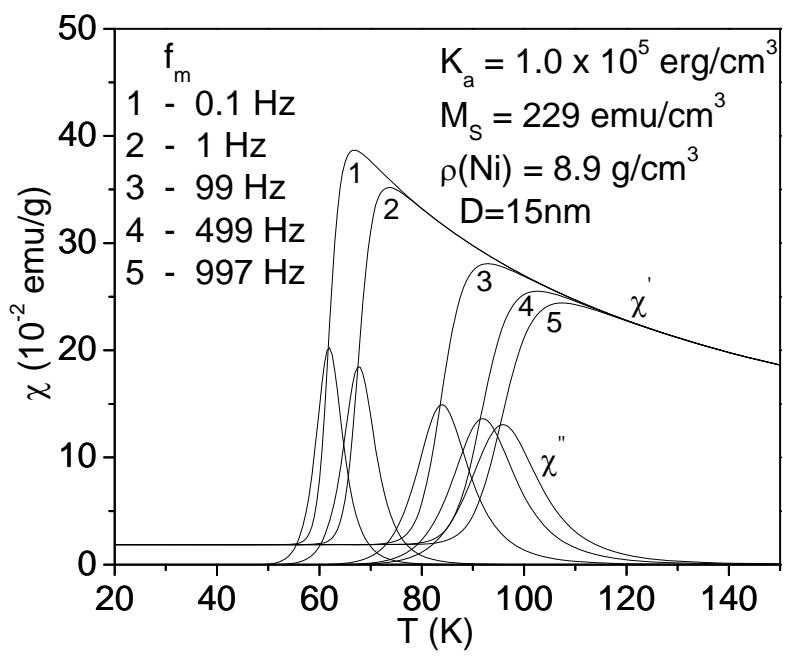

(c)

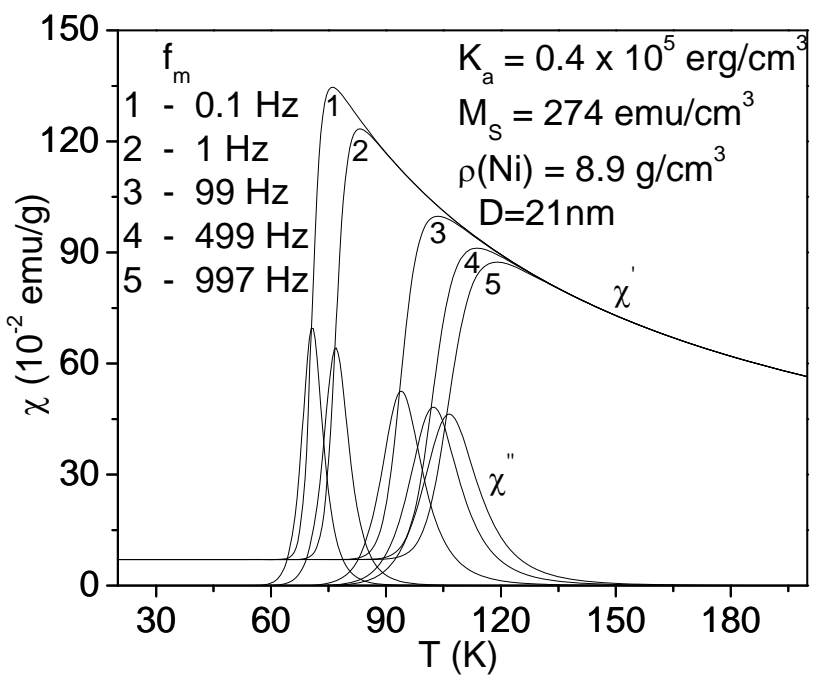

(d)

Fig. 5.19 Simulated $\chi^{\prime}$ and $\chi$ " for sizes $\mathrm{D}=3.8,11.7,15$ and $21 \mathrm{~nm}$. 
Table 5.1 Summary of the experimental and simulated values of $\mathrm{T}_{\mathrm{B}}$ for different frequencies.

\begin{tabular}{|c|c|c|c|c|c|c|}
\hline $\begin{array}{c}\text { frequency } \\
(\mathrm{Hz})\end{array}$ & $\begin{array}{c}\mathrm{D}=3.8 \mathrm{~nm} \\
\mathrm{~T}_{\mathrm{B}}(\mathrm{exp} .)\end{array}$ & $\mathrm{T}_{\mathrm{B}}(\mathrm{sim})$. & $\begin{array}{c}\mathrm{D}=11.7 \mathrm{~nm} \\
\mathrm{~T}_{\mathrm{B}}(\mathrm{exp} .)\end{array}$ & $\mathrm{T}_{\mathrm{B}}(\mathrm{sim})$. \\
\hline 0.1 & 11 & 13 & 37 & 40 \\
\hline 1 & 13 & 14 & & 40 & 44 \\
\hline 99 & 16 & 20 & & 55 & 56 \\
\hline 499 & 18 & 21 & 57 & 64 \\
\hline 997 & & $\mathrm{D}=15 \mathrm{~nm}$ & & & $\mathrm{D}=21 \mathrm{~nm}$ & \\
\hline & $\mathrm{T}_{\mathrm{B}}(\mathrm{exp})$. & $\mathrm{T}_{\mathrm{B}}(\mathrm{sim})$. & & $\mathrm{T}_{\mathrm{B}}(\mathrm{exp})$. & $\mathrm{T}_{\mathrm{B}}(\mathrm{sim})$. \\
\hline 0.1 & & 58 & 62 & & 66 & 71 \\
\hline 1 & & 63 & 68 & & 73 & 77 \\
\hline 99 & & 77 & 84 & & 87 & 94 \\
\hline 499 & & 83 & 96 & & 93 & 103 \\
\hline 997 & & 86 & 96 & & 107 \\
\hline
\end{tabular}




\subsection{Relation between $\chi^{\prime}$ and $\chi^{\prime \prime}$}

We will now test the validity of the relation between $\chi^{\prime}$ and $\chi^{\prime \prime}$ from our results with our data for the $\mathrm{D}=3.8,11.7,15$ and $21 \mathrm{~nm}$ samples. To test the validity of the correlation between $\chi^{\prime \prime}$ and $\partial\left(\chi^{\prime} \mathrm{T}\right) / \partial \mathrm{T}$ in Eq. 5.19, the plots of the experimental $\chi^{\prime \prime}$ and $\mathrm{C} \partial\left(\chi^{\prime} \mathrm{T}\right) / \partial \mathrm{T}$ vs. $\mathrm{T}$ were done. All primary features of experimental $\chi^{\prime \prime}$ such as the frequency and $\mathrm{T}$ dependence are evident in the plots of $\mathrm{C} \partial\left(\chi^{\prime} \mathrm{T}\right) / \partial \mathrm{T}$, except that the peak magnitudes are off by a factor of about 2. This discrepancy is likely related to the approximations made in deriving the magnitude of C [Lundgren et-al, 1981].

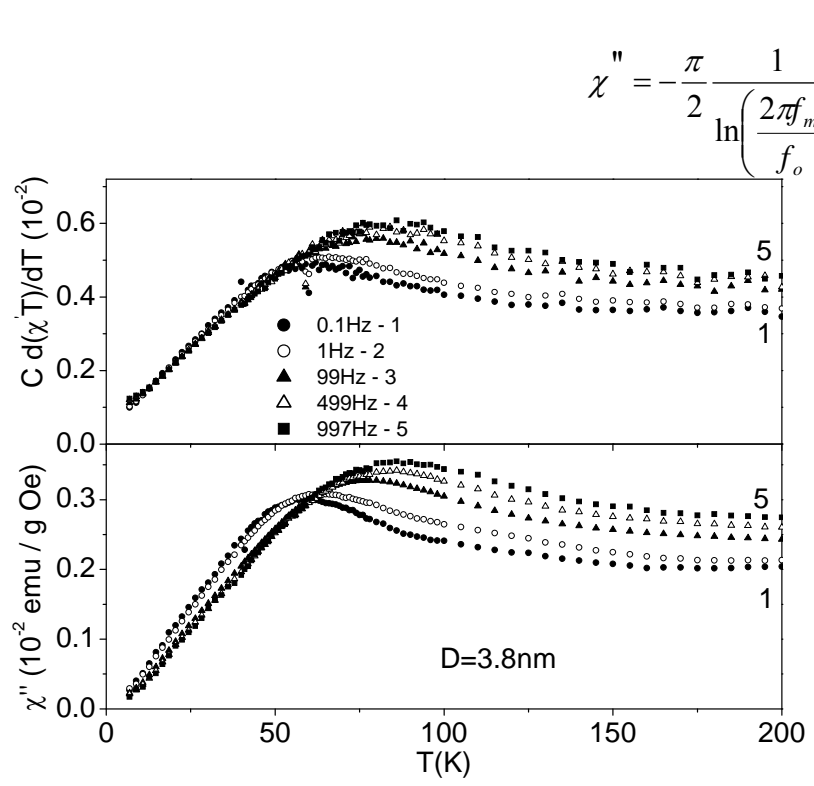

(a)

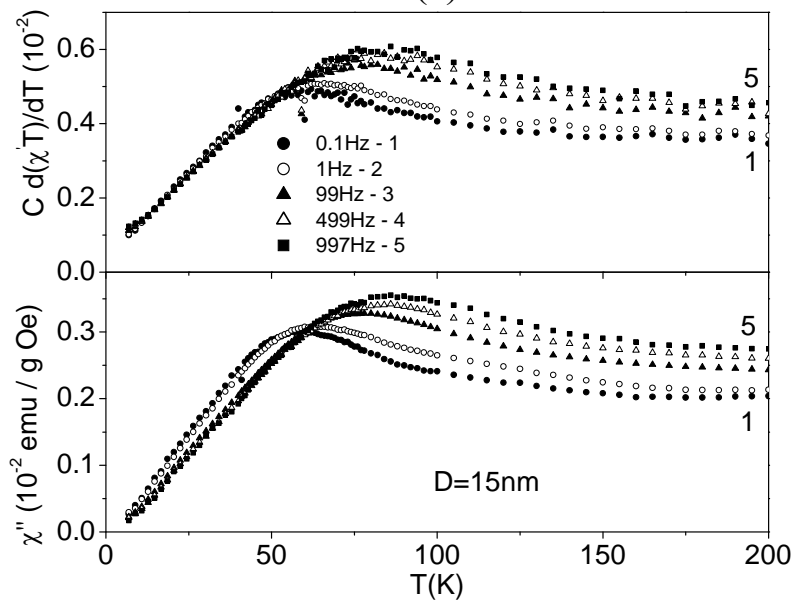

(c)

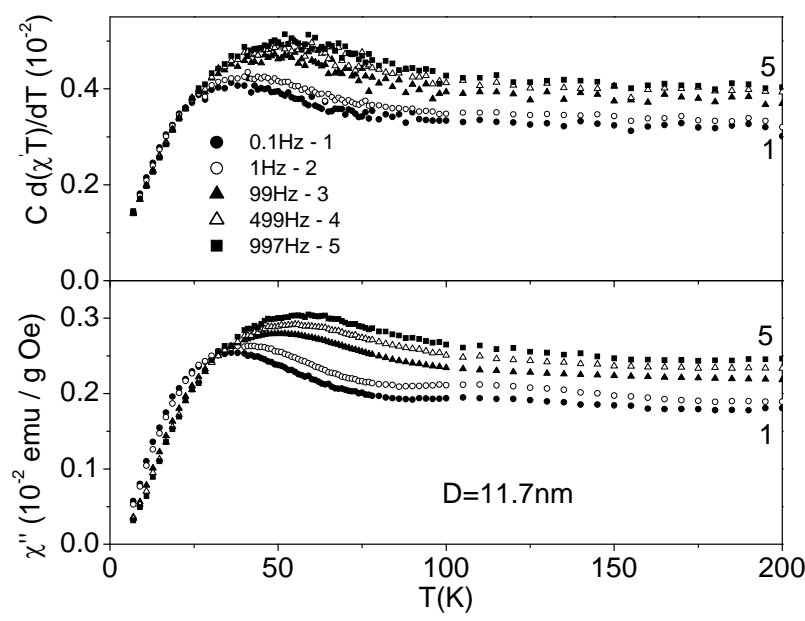

(b)

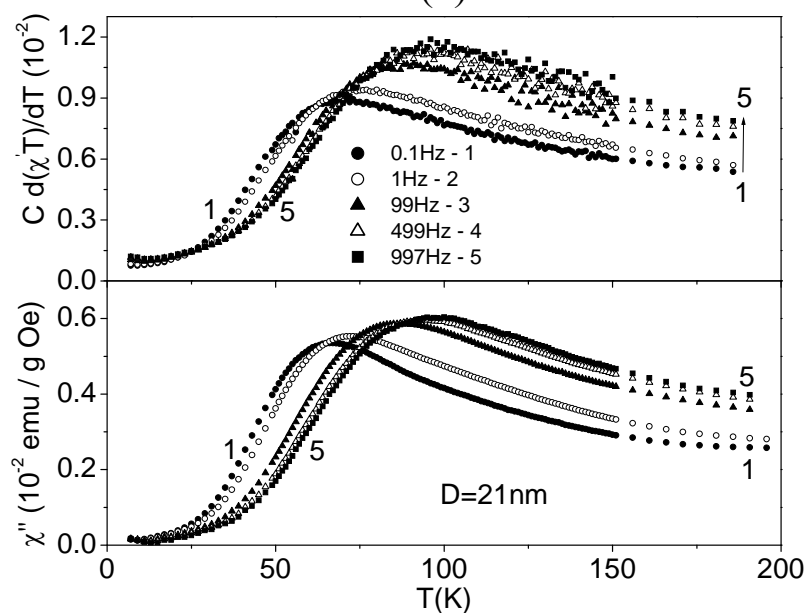

(d)

Fig. 5.20 Plots of experimental $\chi^{\prime \prime}$ vs. T and computed $\mathrm{C} \partial\left(\chi^{\prime} \mathrm{T}\right) / \partial \mathrm{T}$ using $\chi^{\prime}$ vs. T data of the $\mathrm{D}=3.8,11.7,15$ and $21 \mathrm{~nm}$ samples. 


\section{CHAPTER VI}

\section{Experimental results from electron magnetic resonance and discussion}

\subsection{Experimental Details}

The electron magnetic resonance equipment consisted of several major components consisting of a klystron, circulator, cavity, lock-in-amplifier, frequency counter and a Varian magnet with Fieldial.

The klystron was a Varian V-58 klystron which could operate at a microwave frequency range of 8.2 to $12.4 \mathrm{GHz}$ with a maximum power output of $500 \mathrm{~mW}$. This was powered by a Hewlett Packard 716A power supply with Teltronics Model KSLP klystron stabilizer. The principle in the functioning of a klystron is done through a cathode supplying electrons through thermionic emission. This beam of electrons is accelerated to the rf gap (anode) by a resonator voltage where the beam is subjected to an rf electric field which produces a velocity modulated electron beam that slows down and speeds the electrons in the beam. After this, the beam enters drift space where the fast electrons move away from the slower ones behind them and catch up to the slower ones in front of them. The net effect is the formation of groups of electrons which are sent back from the drift space with the help of negative reflector voltage. Upon adjusting the beam, rf and reflector voltage the groups of electrons return in phase to the $\mathrm{rf}$ gap in the process of giving up energy to the resonant cavity.

The generated power travels through the waveguide passing through an isolator which allows them to travel in one direction only and prevents any reflected wave to go through. Part of it is taken out and sent to the microwave frequency counter 5342A to measure the frequency. The power then goes through the circulator where the function of the circulator is to allow the wave to enter through one arm reach the resonant cavity through a slide screw tuner in the second arm. Upon reaching the cavity the Power absorbed is modulated at a modulation frequency of $100 \mathrm{kHz}$ provided by an oscillator 204C/204D with the superimposed magnetic field. The reflected wave from the cavity goes out through the third arm of the circulator to reach the detector. Before reaching the detector the waves pass through a directional coupler whose purpose is to take out a part of the microwave energy without disturbing the main flow and send the reflected power 
to the oscilloscope and to the Teltronics klystron frequency stabilizer. The stabilizer provides a correction voltage used to correct the klystron reflector voltage. The power reaches the crystal detector and the signal from the crystal detector is transmitted to a Princeton Applied Research Model 5204 lock-in-amplifier where it is phase detected with the reference $100 \mathrm{kHz}$ signal. The phase detected signal yields the derivative of the power absorbed with respect to the applied static magnetic field.

The Electron Magnetic Resonance (EMR) signal was obtained by taking a small specimen of the sample and inserting it in a long quartz tube. The tube was then placed between the poles of an electromagnet inside a microwave cavity with a $\mathrm{TE}_{102}$ mode, where a DC field could be swept through the sample. An alternating AC field from the klystron was applied perpendicular to it at frequency of $9.28 \mathrm{GHz}$. The DC field had a sweep from 0-10000 Oe but could be set to scan a specified range to obtain an amplified signal. Low temperature EMR could also be studied with temperature starting from $\mathrm{T}=5$ $\mathrm{K}$ to room temperature using an oxford instrument cryostat. In order to obtain low temperatures, an external helium transfer tube was connected to the sample chamber for the helium to flow through to the chamber in order to reach $5 \mathrm{~K}$. Temperature variation could be achieved through controlling the flow of liquid helium reaching the chamber and a heater. This could be done by a variable switch located on the liquid helium transfer tube.

Principles of magnetic resonance were discussed in some detail in chapter 4, where the resonance condition is obtained when the oscillating field of angular frequency $\omega$ with amplitude $\mathrm{H}_{1}$ causes transitions between the Zeeman levels. This corresponds to the power $(\mathrm{P})$ absorbed by the sample which is proportional to $\chi^{\prime \prime}$, the out of phase component of the AC susceptibility:

$$
P=\frac{1}{2} H_{1}{ }^{2} \omega \chi "
$$

Temperature variation of EMR studies from 5 to $300 \mathrm{~K}$ (see Figs. 6.4 to 6.8) were done for the 3.8, 11.7, 15, 21 and $23 \mathrm{~nm}$ particles with microwave frequency of 9.28 GHz. In order to interpret the EMR spectra, it is assumed that the Ni NPs are sufficiently separated to ignore interparticle interactions or any interaction with the silica matrix [Biasi et al, 1978]. Interaction with matrix can be neglected because of its diamagnetic 
character. Interactions with the other particles can be ignored assuming that the particles are far enough that they do not interact with each other. The only interaction that occurs is with the particle and the applied field. Skin depth effects due to conductivity $(\sigma)$ effects can be regarded as negligible if the particle size is smaller that the skin depth. The skin depth $(\delta)$ can be obtained from the Eq. 6.2 [Poole, 1983]:

$$
\delta=\sqrt{\frac{2}{\omega \mu \sigma}} \text { meters }
$$

For $\mathrm{Ni}, \delta=63 \mathrm{~nm}$ for $\mu(\mathrm{Ni})=7.54 \times 10^{-4} \mathrm{Hm}^{-1}, \sigma(\mathrm{Ni})=1.15 \times 10^{7}(\Omega \mathrm{m})^{-1}$ and $(\omega / 2 \pi)=9.28$ GHz. Since all NPs are smaller that $63 \mathrm{~nm}$, skin depth effects are negligible for the samples used in our studies.

For magnetic NPs, two sources of energy that are competing with each other are thermal energy and magnetic anisotropy energy. Thermal energy tends to average out the effect of anisotropy energy which both broadens and shifts the line. When the thermal energy falls below the magnetic anisotropy energy the peak position shifts to lower fields. This can be explained on the basis of Eq. 6.3 given below for an effective anisotropy field $\mathrm{H}_{\text {eff: }}$

$$
H_{r}=\left(\frac{\omega_{o}}{\gamma}\right)-H_{\text {eff }}
$$

The effective $g$ value can be calculated from the resonance field $\mathrm{H}_{\mathrm{r}}$ given by the equation below:

$$
g=\frac{h f}{\mu_{B} H_{r}}
$$

where $h$ is the Planck's constant, $f$ is the microwave frequency and $\mu_{\mathrm{B}}$ is the Bohr magneton. The linewidth $\Delta \mathrm{H}$ was deduced from the peak-to-peak distance between the minimum and the maximum of the derivative of the absorption signal. 


\subsection{Temperature and Size Dependence of $\mathbf{H}_{\mathrm{eff}}$}

The anisotropy energy in bulk samples is given by:

$$
E_{a}=\alpha K=\alpha H_{A} M
$$

where $\alpha$ is a function that depends on the angle $(\theta)$ between the magnetization and easy axis, $\mathrm{K}$ is the bulk anisotropy constant and $\mathrm{H}_{\mathrm{A}}$ is the anisotropy field. For small particles the average is taken over a distribution of all angles $(\theta)$ so the anisotropy energy becomes [Biasi et al, 1978]:

$$
\begin{aligned}
E_{a} & =\alpha\langle K\rangle=\alpha H_{A}{ }^{S P}\langle M\rangle \\
H_{A}{ }^{S P} & =\langle K\rangle /\langle M\rangle
\end{aligned}
$$

where $<\mathrm{K}>=\mathrm{K}<\mathrm{P}_{\mathrm{n}}(\cos \theta)>, \mathrm{P}_{\mathrm{n}}$ is the $\mathrm{n}^{\text {th }}$ order Legendre polynomial with $\theta$ as the angle between the magnetic moment and magnetic field and $n=2$ for axial and $n=4$ for cubic symmetry. $<\mathrm{M}>=\mathrm{I}_{\mathrm{S}}<\cos \theta>$ where $\mathrm{I}_{\mathrm{s}}$ is the magnetic moment of the particles. Upon substitution of $<\mathrm{K}>$ and $<\mathrm{M}>$ in Eq. 6.7 we get:

$$
{\frac{H_{A}}{H_{A}}}^{S P}=\frac{\left\langle P_{n}(\cos \theta)\right\rangle}{\langle\cos \theta\rangle} \text { with } H_{A}=K / I_{S}
$$

Substituting for $<\mathrm{P}_{\mathrm{n}}(\cos \theta)>$ and $<\cos \theta>$ (see appendix III) yields:

$$
H_{A}^{S P}(\text { Axial })=H_{A} \frac{1-3 x^{-1} \operatorname{coth}(x)+3 x^{-2}}{\operatorname{coth}(x)-x^{-1}}
$$

for axial symmetry and

$$
H_{A}^{S P}{ }_{(\text {Cubic })}=H_{A} \frac{1-10 x^{-1} \operatorname{coth}(x)+45 x^{-2}-105 x^{-3} \operatorname{coth}(x)+105 x^{-4}}{\operatorname{coth}(x)-x^{-1}}
$$

for cubic symmetry (see appendix III). Here $x=\mu_{P} H / k_{B} T$ and $H_{A}=K / M$ where $K$ is the anisotropy constant, $\mathrm{M}$ is the sample magnetization and $\mu_{\mathrm{P}}$ is the magnetic moment per particle. The variation of $\mathrm{H}_{\mathrm{A}}{ }^{\mathrm{SP}} / \mathrm{H}_{\mathrm{A}}$ with $\mathrm{x}$ is shown in the Fig. 6.1. Fig 6.2 shows a direct relation between the effective anisotropy vs. temperature for different particle sizes. Since the anisotropy of $\mathrm{Ni}$ is cubic, the detailed calculations are presented for the cubic system only. From Fig. 6.2, it is clear that $\mathrm{H}_{\mathrm{A}}{ }^{\mathrm{SP}}$ increases with increase in the size of the NPs at a given temperature. Also, for a given particle size, $\mathrm{H}_{\mathrm{A}}{ }^{\mathrm{SP}}$ increases with decrease in temperature, the largest relative change occurring for the smallest particles. It is noted 
that decrease in $\mathrm{H}_{\mathrm{A}}^{\mathrm{SP}}$ with increasing temperature is due to averaging effects of the thermal energy. We will compare those predictions with the experimental results presented next.

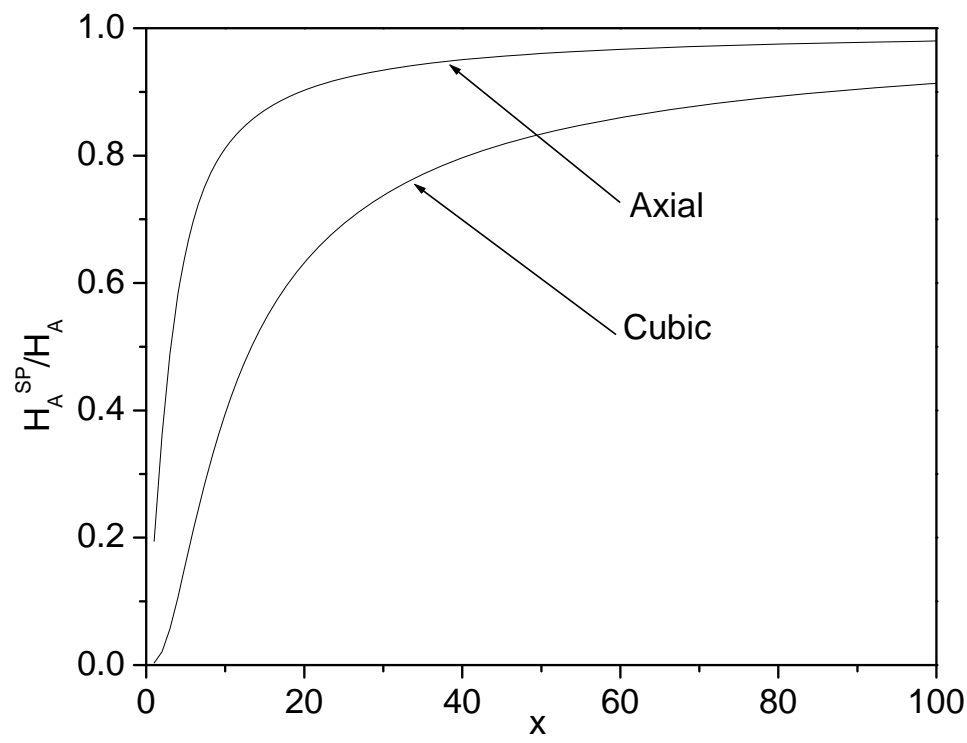

Fig. 6.1 Variation of anisotropy field with cubic and axial symmetries

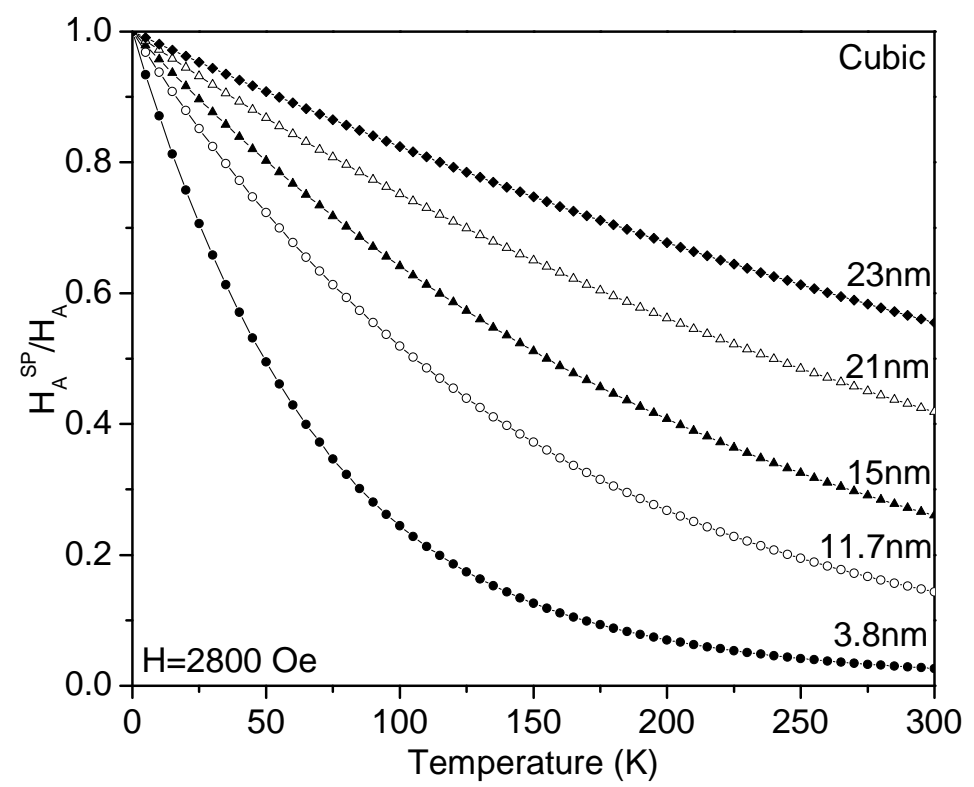

Fig. 6.2 Variation of anisotropy field with temperature for $\mathrm{Ni}$ (cubic) for different particle sizes. 


\subsection{General Features of the Observed EMR lines}

Comparison of the observed EMR spectra in the five different size samples of $\mathrm{Ni} / \mathrm{SiO}_{2}$ at $5 \mathrm{~K}$ is shown in Fig. 6.3. The broadening $(\Delta \mathrm{H})$ of the lines was determined by taking the difference between the extremes and the resonance position $\left(\mathrm{H}_{\mathrm{r}}\right)$ was determined by taking the mid-field between the extremes. There were some difficulties involved in obtaining the exact peak positions from the spectra due to overlapping of the EMR lines. For line 3 which is not well resolved only the peak position has been plotted. In Fig. 6.3, the three lines are denoted as 3,2 and 1 with increasing magnetic field plotted. The details of the temperature variation of the lines are presented below.

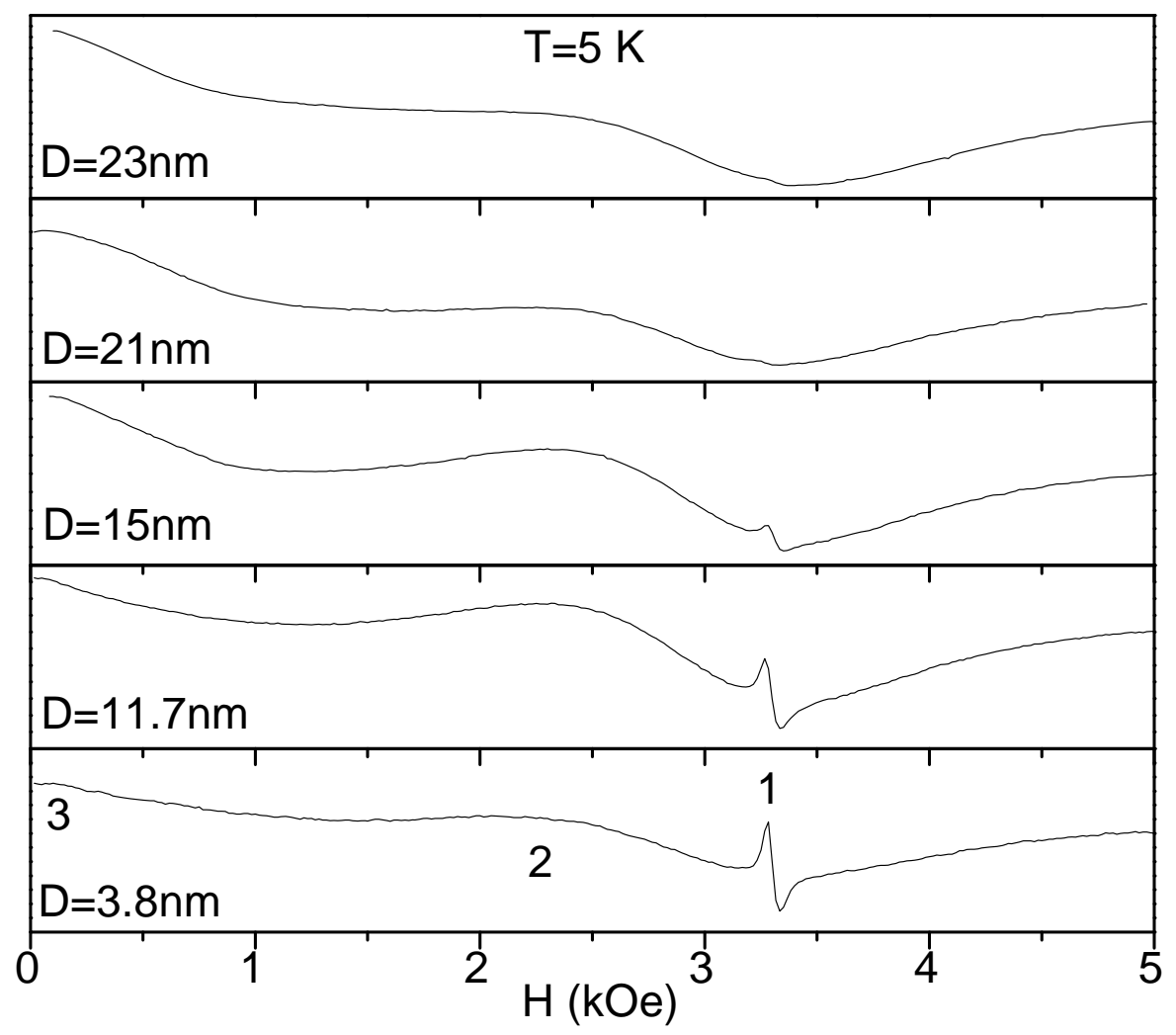

Fig. 6.3 EMR lines for $\mathrm{D}=3.8,11.7,15,21$ and $23 \mathrm{~nm}$ at $\mathrm{T}=5 \mathrm{~K}$.

The temperature variation of the observed EMR scans are plotted in Figs. 6.4 to 6.8 for particle sizes $\mathrm{D}=3.8,11.7,15,21$ and $23 \mathrm{~nm}$ respectively. In all these cases, line 2 
is the line with the most intensity at $300 \mathrm{~K}$. For this reason, this line is interpreted to originate from majority of the particles with the size distribution shown in the TEM micrographs of Figs. 2.10, 2.11 and 2.12 in chapter 2 with the average sizes listed above. As the temperature is lowered, line 3 emerges at lower fields and line 1 at $\mathrm{H}_{\mathrm{r}} \simeq 3000$ Oe. An attempt was made to resolve these lines through computer programs. However the fits were not entirely satisfactory and this analysis is not presented here. The origin of line 3 is believed to be from larger particles or cluster of particles since it is shifted the most from the $\mathrm{g} \simeq 2$ position expected for Ni. As shown in the analysis of Fig. 6.2, the larger particles have higher anisotropy and hence higher lineshifts and linewidths following the predictions of Eq. 6.3.

Through extensive investigations described later, the origin of line 1 is attributed to defects in $\mathrm{SiO}_{2}$ matrix. Since $\mathrm{H}_{\mathrm{r}}$ and $\Delta \mathrm{H}$ for this line do not vary with change in temperature, this line is not due to very small Ni particles as some authors have argued [Kliava et al, 1999]. If this line was from small Ni NPs, then as shown in Fig. 6.2, the anisotropy for this should be highly temperature dependent leading to strong temperature dependence of $\mathrm{H}_{\mathrm{r}}$ and $\Delta \mathrm{H}$. This is not observed. In addition, we observe a line in undoped $\mathrm{SiO}_{2}$ prepared similarly with identical temperature dependence of $\mathrm{H}_{\mathrm{r}}, \Delta \mathrm{H}$ and the line intensity $\mathrm{I}=(\Delta \mathrm{H})^{2} l$ where $l$ is the peak-to-peak height. These results on line 1 are described in detail later.

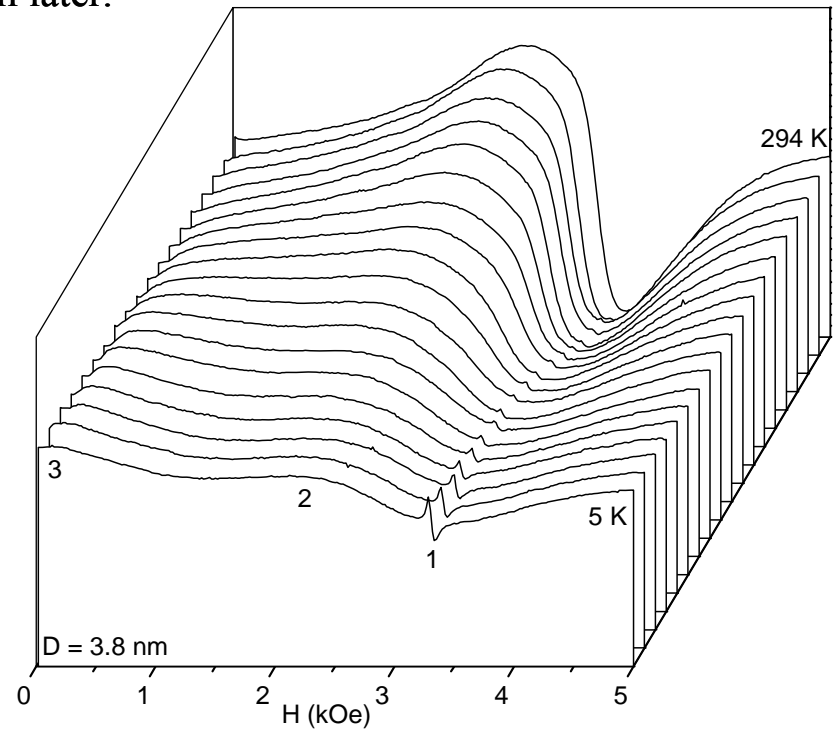

Fig. 6.4 EMR lines for $\mathrm{D}=3.8 \mathrm{~nm}$ for temperatures $\mathrm{T}=5,10,16,20,31,40,54,68,83,93$, $107,121,133,150,170,190,204,217,240,260,280$ and $294 \mathrm{~K}$. 


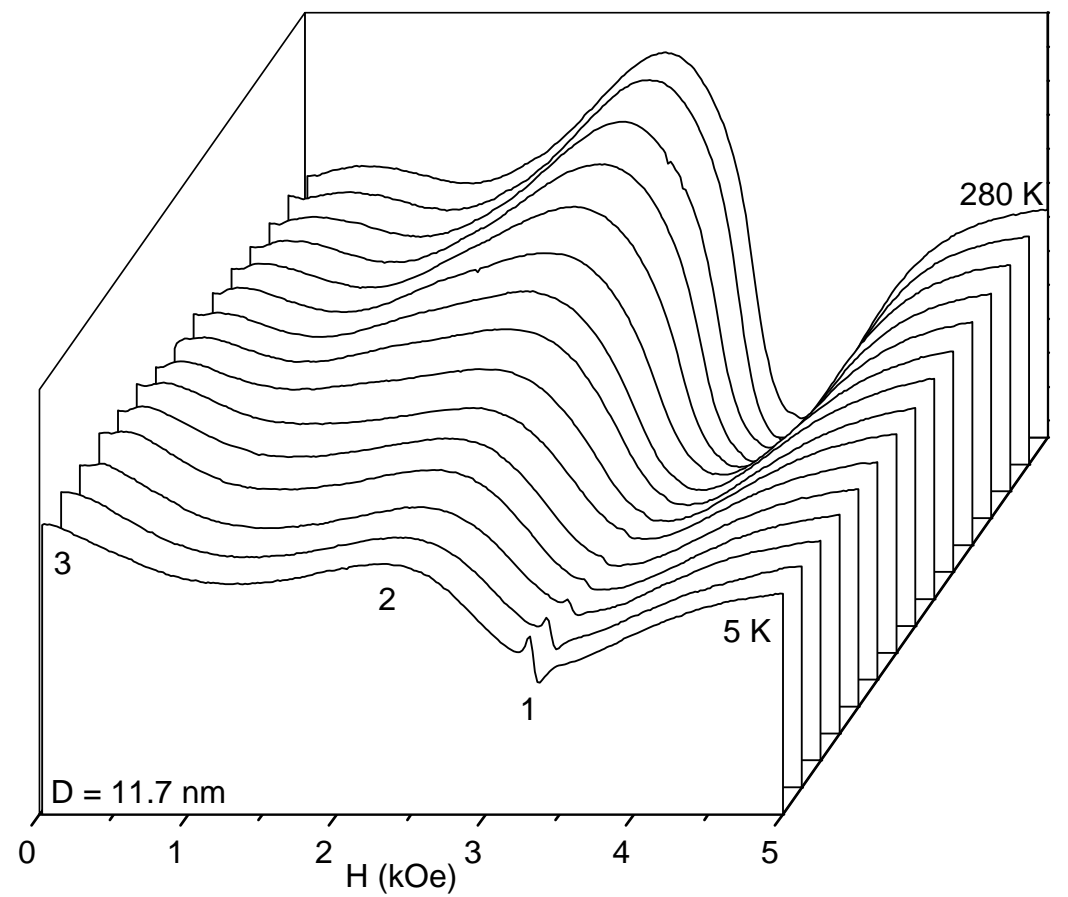

Fig. 6.5 EMR lines for $\mathrm{D}=11.7 \mathrm{~nm}$ for temperatures $\mathrm{T}=5,9,14,32,66,80,90,114,134$, $150,170,193,215,240,265$ and $280 \mathrm{~K}$.

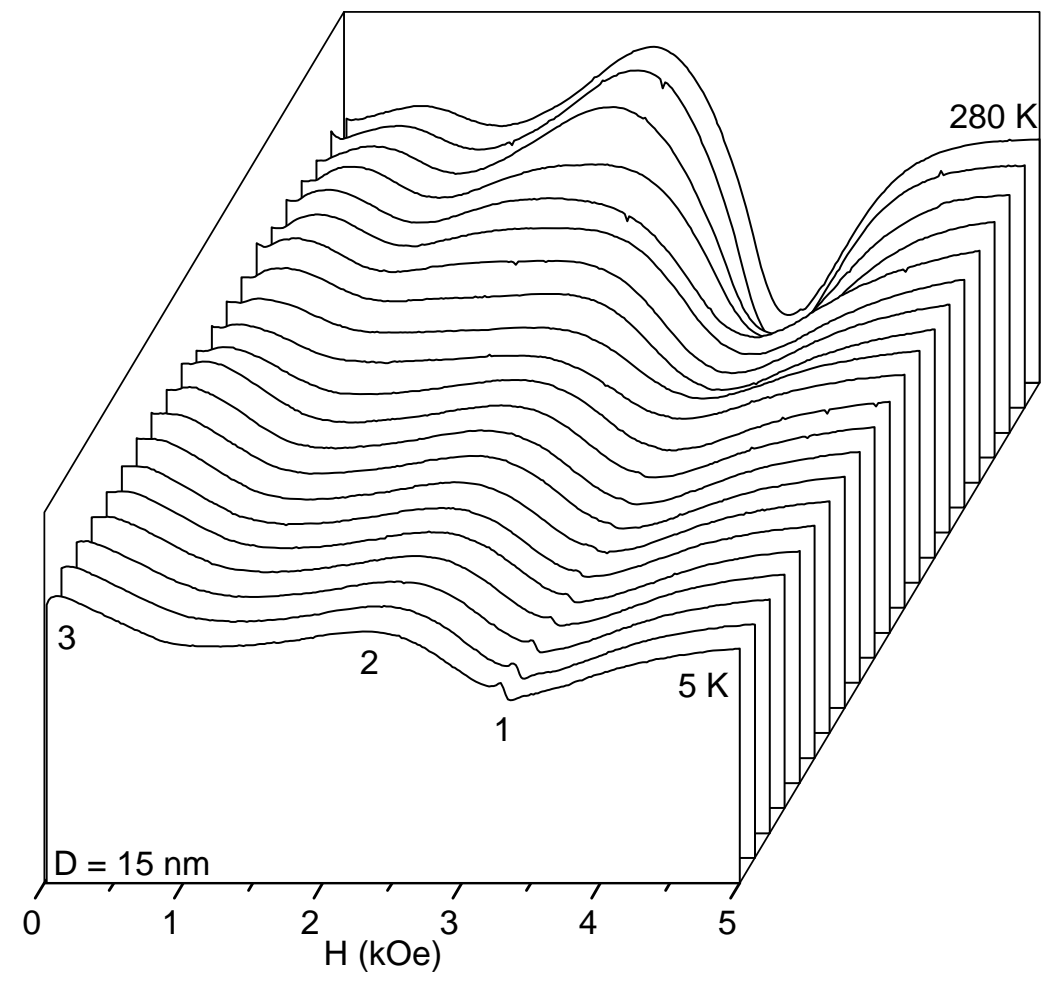

Fig. 6.6 EMR lines for $\mathrm{D}=15 \mathrm{~nm}$ for temperatures $\mathrm{T}=5,8,14,20,25,29,35,43,48,55$, $75,90,105,115,127,140,155,170,210,250$ and $280 \mathrm{~K}$. 


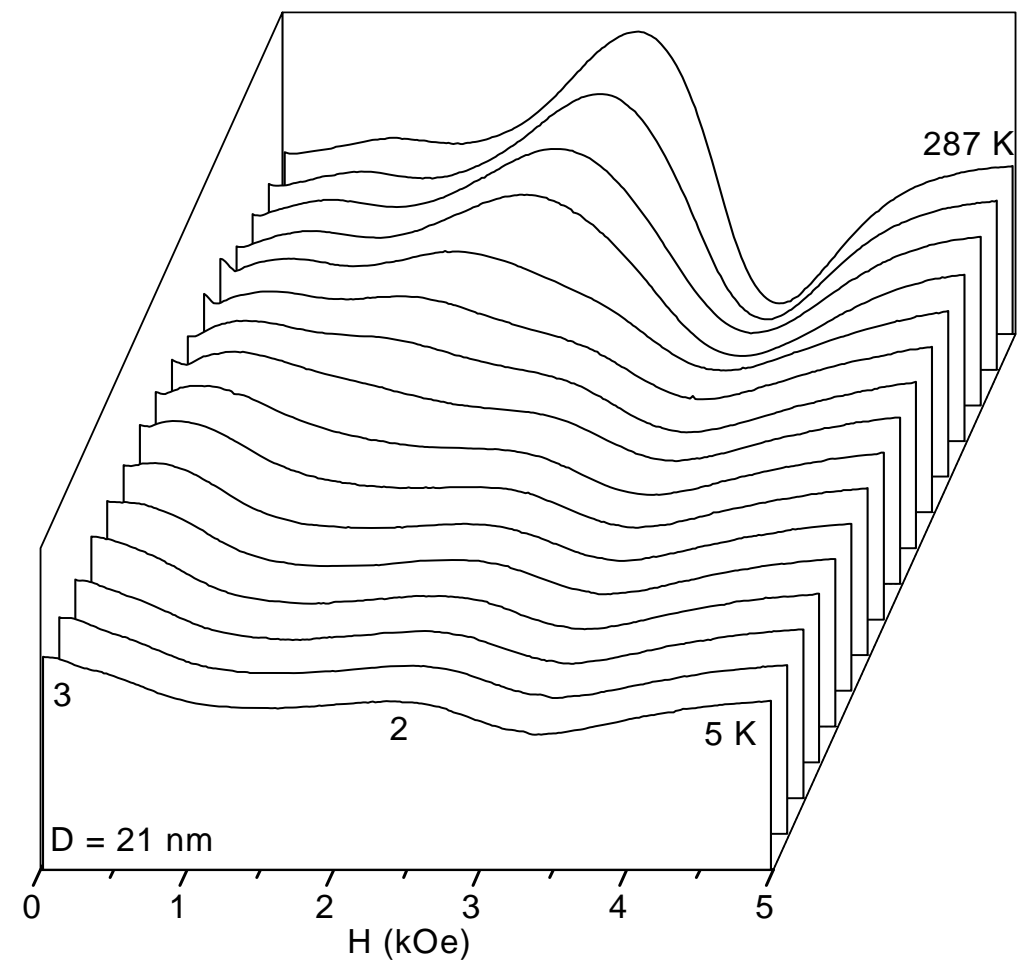

Fig. 6.7 EMR lines for $\mathrm{D}=21 \mathrm{~nm}$ for temperatures $\mathrm{T}=5,5,13,18,28,45,58,70,85,95$, $110,122,132,147,170,190,230$ and $287 \mathrm{~K}$.

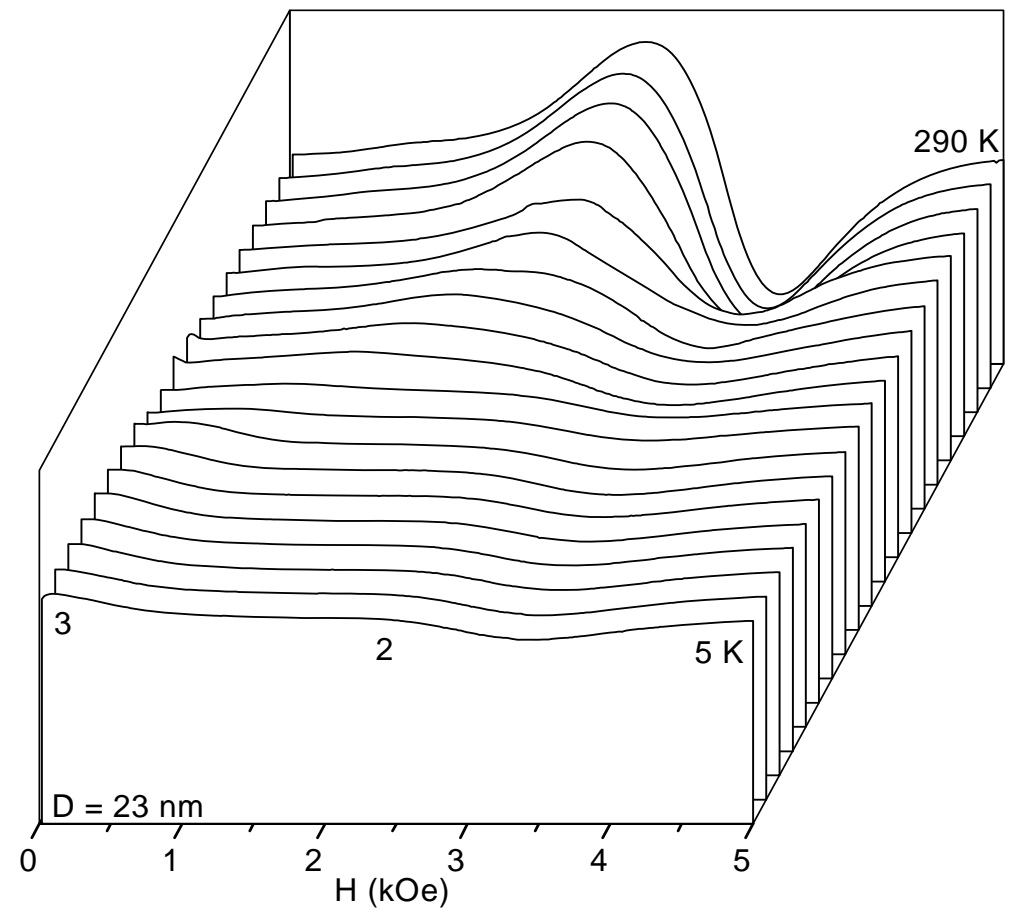

Fig. 6.8 EMR lines for $\mathrm{D}=23 \mathrm{~nm}$ for temperatures $\mathrm{T}=5,10,15,20,25,33,40,53,80,95$, $117,130,145,160,180,200,220,235,260$ and $290 \mathrm{~K}$. 


\subsection{Temperature dependence of Line 3}

Since only a part of line 3 is observed, it is difficult to measure accurate $\mathrm{H}_{\mathrm{r}}$ and $\Delta \mathrm{H}$ for this line. Therefore we have used the position of the peak of line 3 as its approximate $\mathrm{H}_{\mathrm{r}}$. The temperature dependence of this $\mathrm{H}_{\mathrm{r}}$ for line 3 is shown in Fig. 6.9. For all particles sizes, the line shifts to lower fields with decrease in temperature. This is consistent with the predictions of Eq. 6.3 if $\mathrm{H}_{\mathrm{eff}}$ increases with decrease in temperature. This is indeed predicted by the analysis shown in Fig. 6.2.

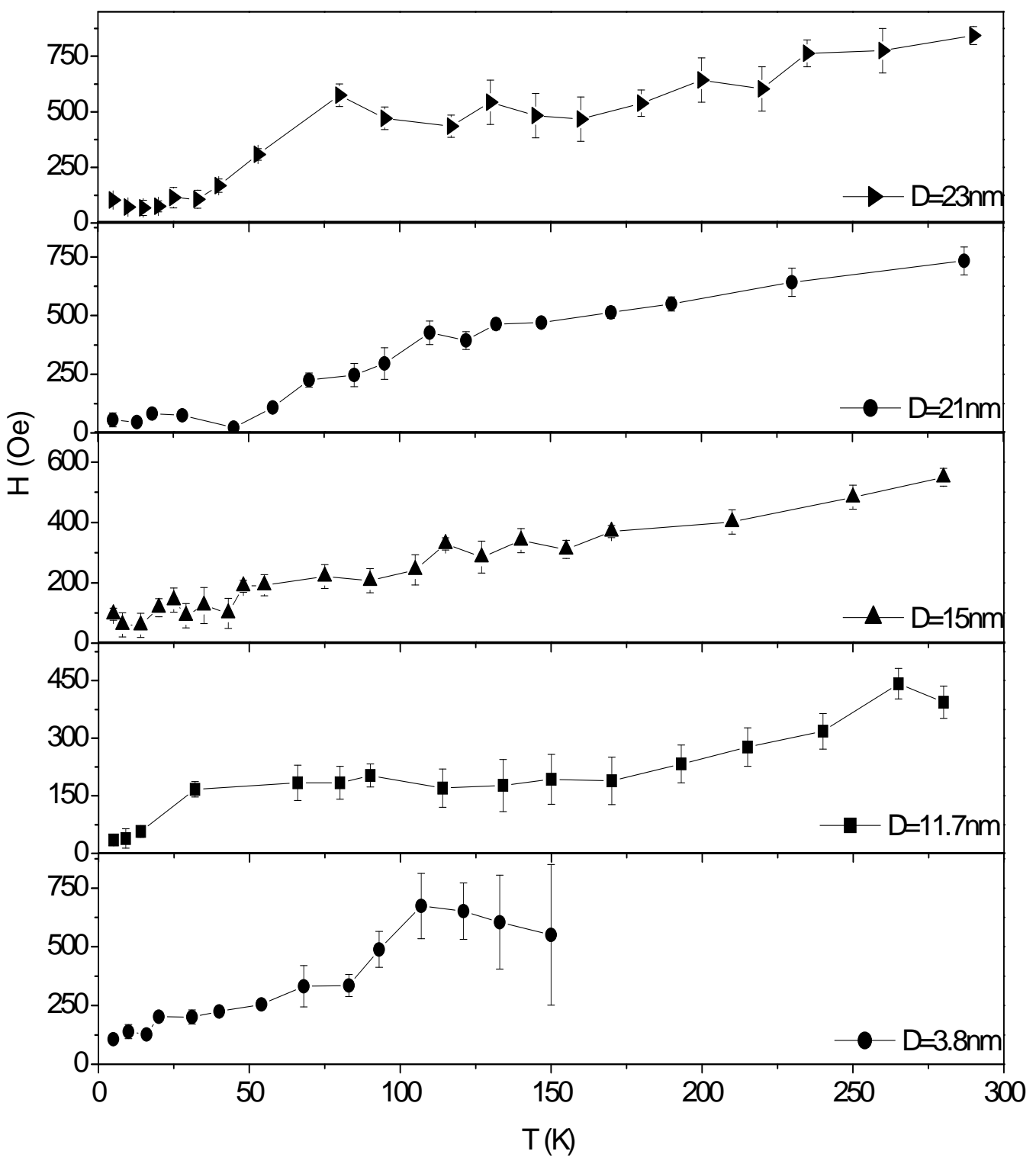

Fig.6.9 Peak position of line 3. 


\subsection{Temperature dependence of Line 2}

In this section, the temperature dependence of $\mathrm{H}_{\mathrm{r}}, \Delta \mathrm{H}$ and line intensity (I) are considered together for each particle size. For the $\mathrm{D}=3.8 \mathrm{~nm} \mathrm{Ni} / \mathrm{SiO}_{2}$ sample, such plots are shown in Fig. 6.10. Note that both $\mathrm{I}$ and $\Delta \mathrm{H}$ increase with decrease in temperature, reaching maximum values near $100 \mathrm{~K}$ and then rapidly decrease with further lowering of the temperature. The resonance field $\mathrm{H}_{\mathrm{r}}$ decreases (or equivalently lineshift increases) with decrease in $\mathrm{T}$, reaching a minimum around $100 \mathrm{~K}$. These signatures are characteristics of a blocking temperature but now measured by EMR.

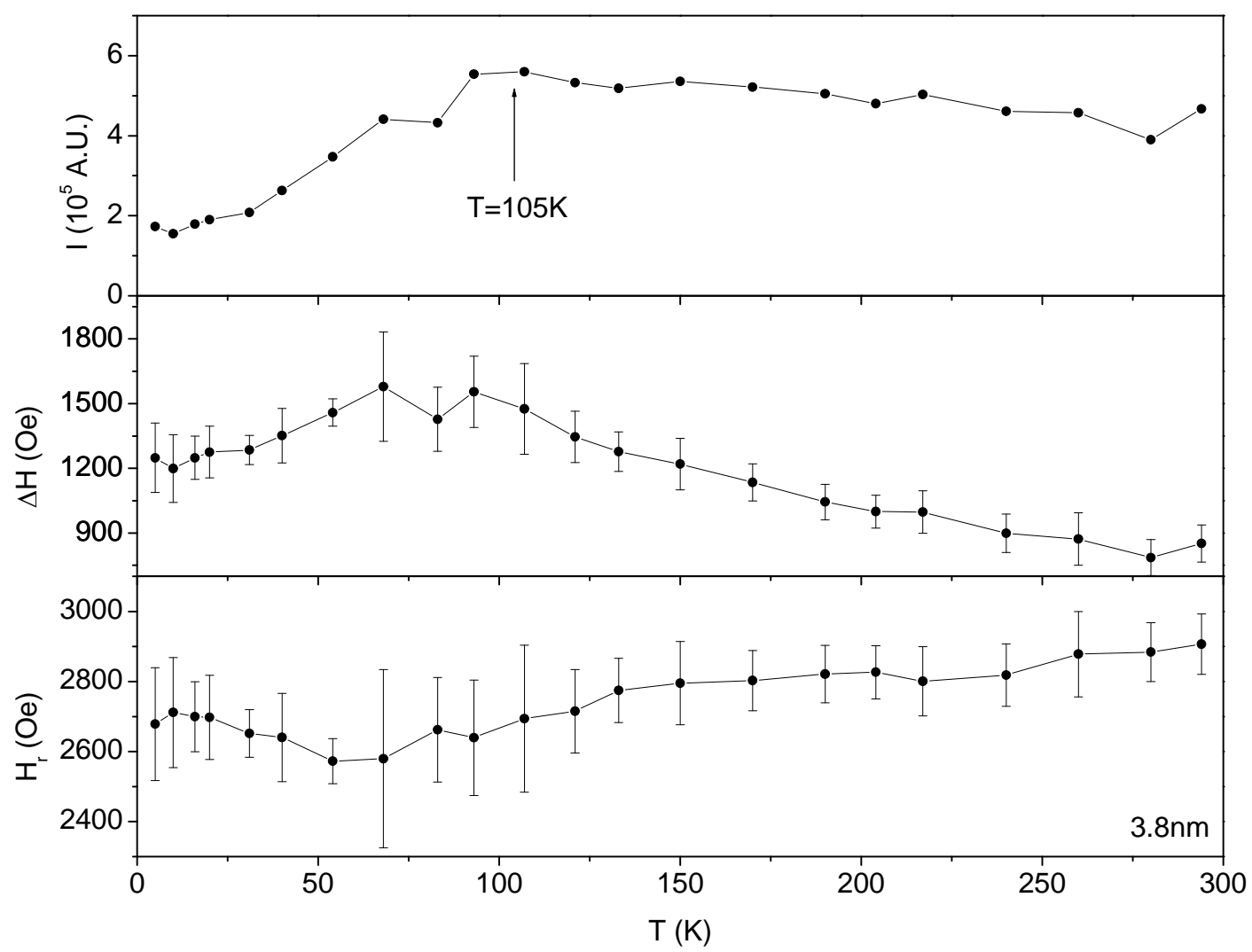

Fig. 6.10 Temperature dependence of $\mathrm{H}_{\mathrm{r}}, \Delta \mathrm{H}$ and $\mathrm{I}$ of line 2 for $\mathrm{D}=3.8 \mathrm{~nm}$. 


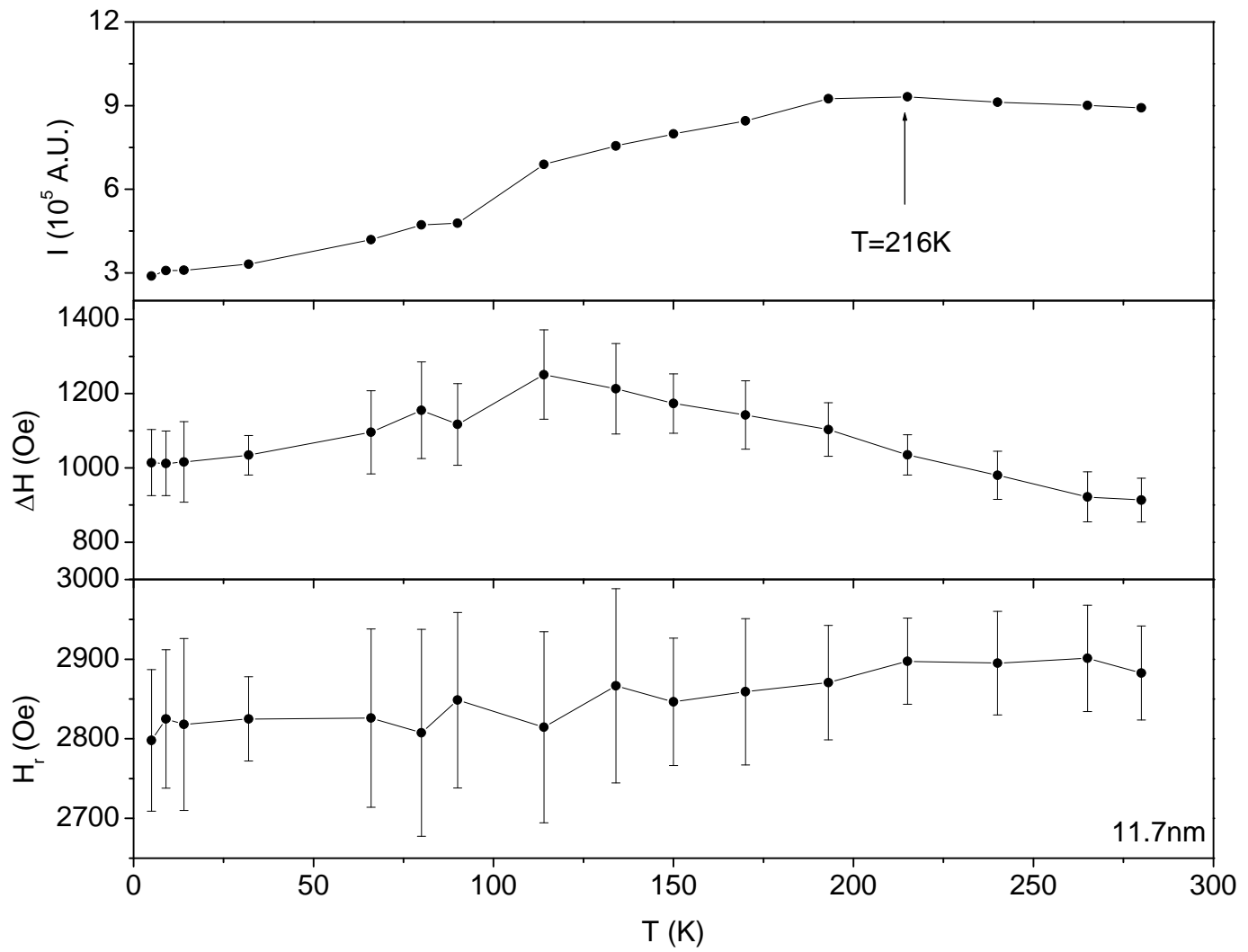

Fig. 6.11 Temperature dependence of $\mathrm{H}_{\mathrm{r}}, \Delta \mathrm{H}$ and $\mathrm{I}$ of line 2 for $\mathrm{D}=11.7 \mathrm{~nm}$.

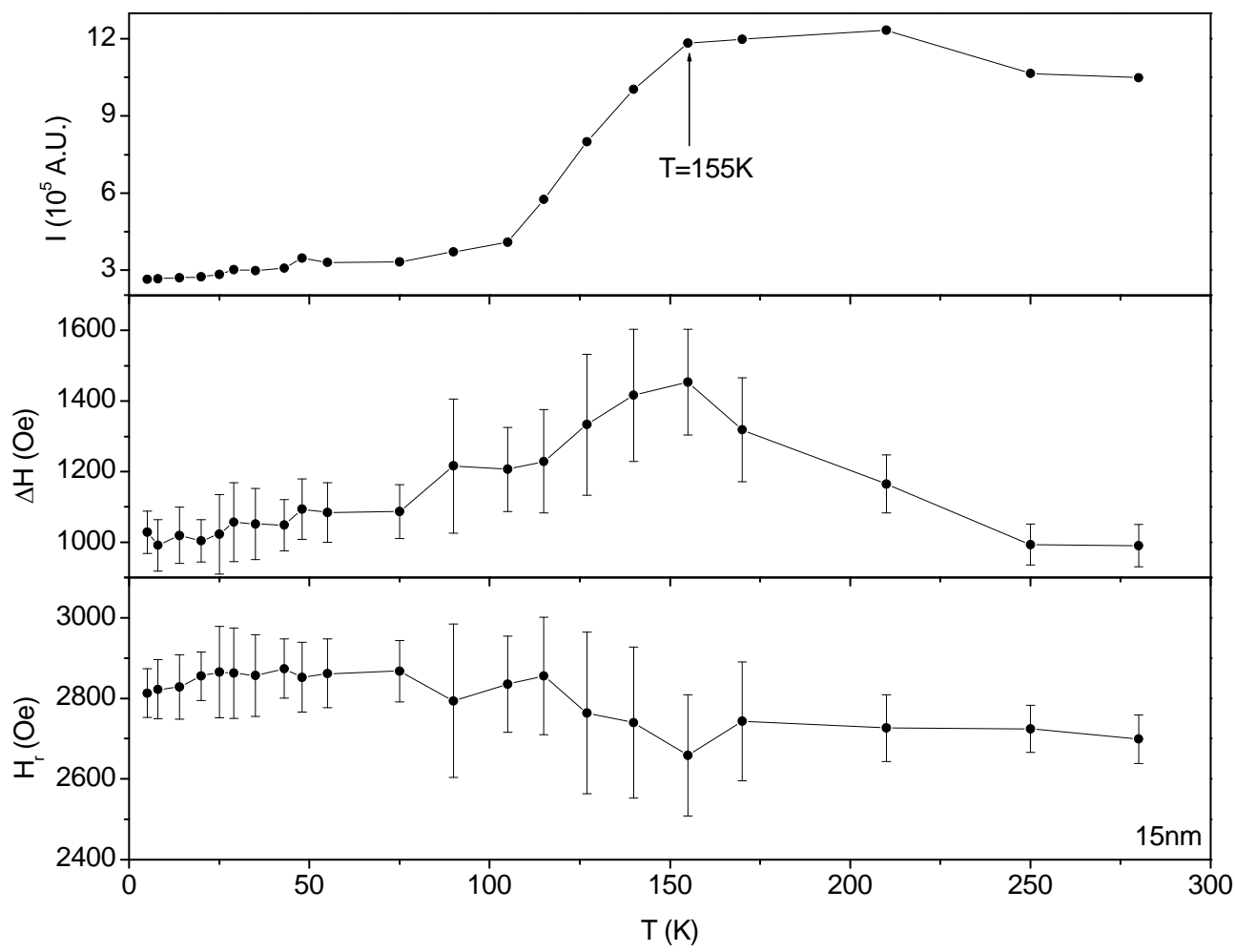

Fig. 6.12 Temperature dependence of $\mathrm{H}_{\mathrm{r}}, \Delta \mathrm{H}$ and $\mathrm{I}$ of line 2 for $\mathrm{D}=15 \mathrm{~nm}$. 


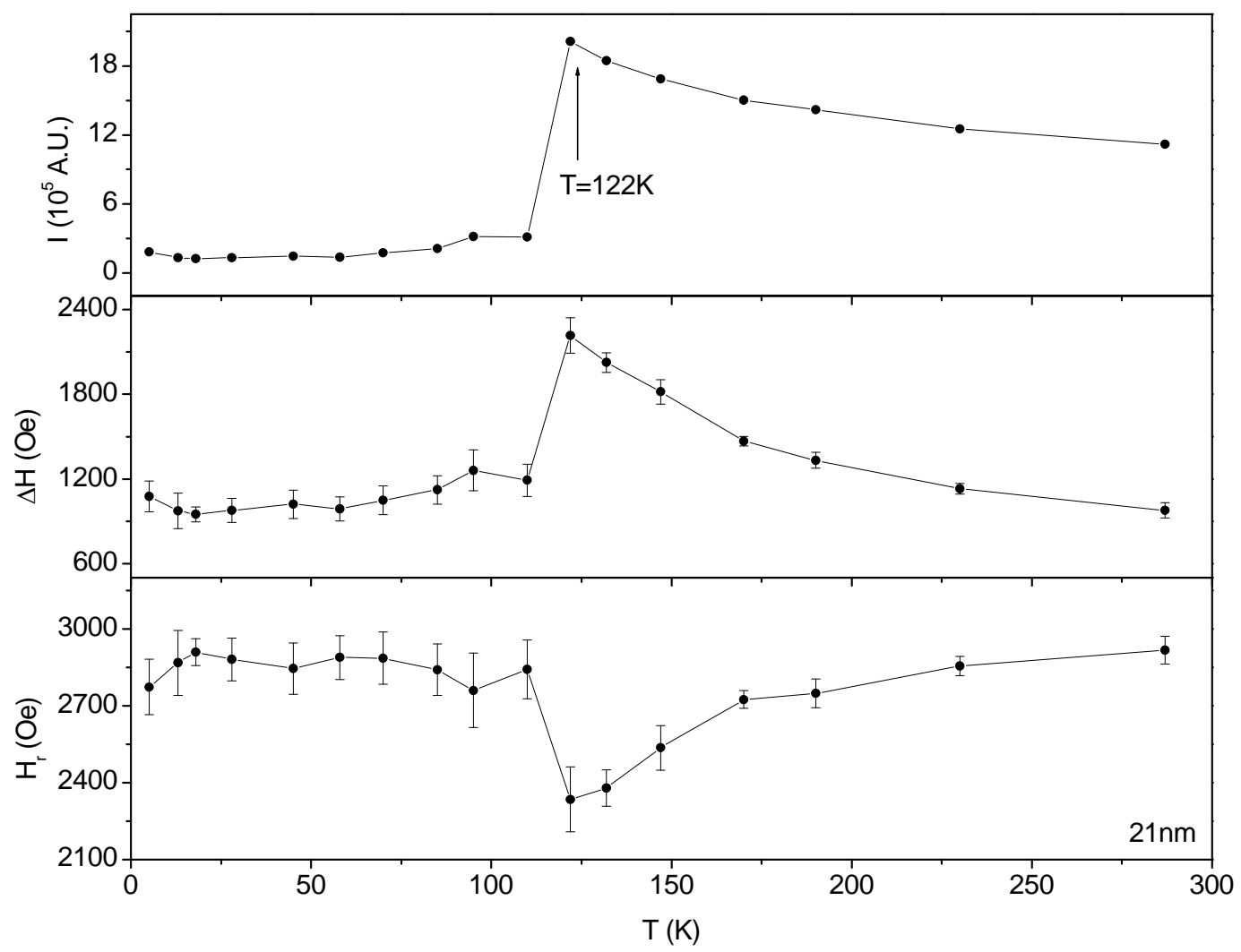

Fig. 6.13 Temperature dependence of $\mathrm{H}_{\mathrm{r}}, \Delta \mathrm{H}$ and $\mathrm{I}$ of line 2 for $\mathrm{D}=21 \mathrm{~nm}$.

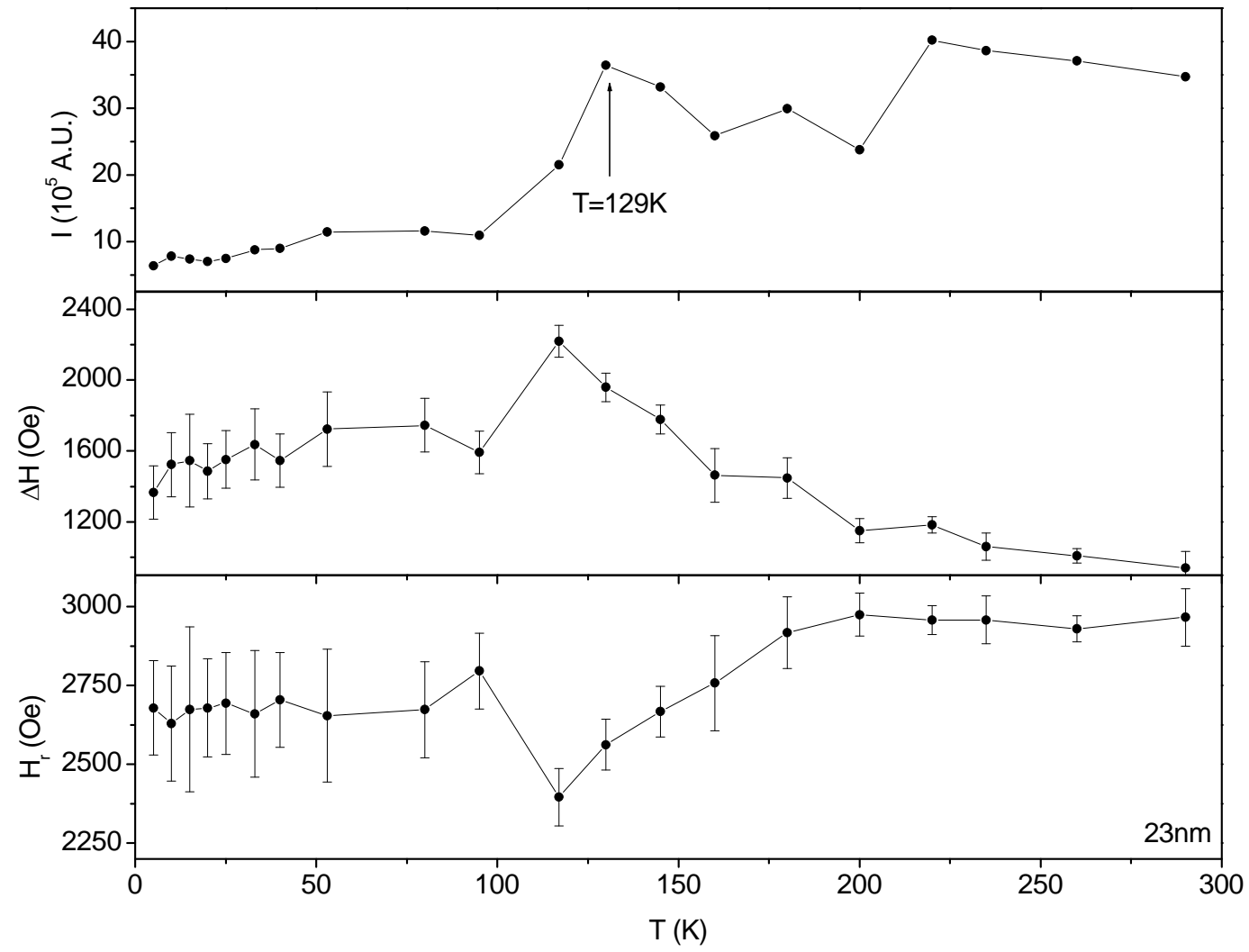

Fig. 6.14 Temperature dependence of $\mathrm{H}_{\mathrm{r}}, \Delta \mathrm{H}$ and $\mathrm{I}$ of line 2 for $\mathrm{D}=23 \mathrm{~nm}$. 
So $T_{B}(E M R) \simeq 100 \mathrm{~K}$ is deduced from this data on $\mathrm{D}=3.8 \mathrm{~nm}$. Recall that $\mathrm{T}_{\mathrm{B}}(\mathrm{M}) \simeq 20 \mathrm{~K}$ was determined for this sample using low field SQUID measurements. So $\mathrm{T}_{\mathrm{B}}(\mathrm{EMR}) /$ $T_{B}(M) \simeq 5$. As shown earlier in Eq. 3.26, $T_{B}$ is given by:

$$
T_{B}=\frac{T_{a}}{\ln \frac{f_{o}}{f_{m}}}
$$

leading to

$$
\frac{T_{B}(E M R)}{T_{B}(M)}=\frac{\ln \left(\frac{f_{o}}{f_{m}(M)}\right)}{\ln \left(\frac{f_{o}}{f_{m}(E M R)}\right)}
$$

Using $f_{m}(E M R)=9.28 \times 10^{9} \mathrm{~Hz}, f_{m}=1 \mathrm{~Hz}$ for $T_{B}(M)$ and $f_{o}=1.82 \times 10^{10} \mathrm{~Hz}$ determined earlier, we get $T_{B}(E M R) / T_{B}(M) \simeq 35$, a factor of about 7 larger than the experimental value of 5. It is very likely that this discrepancy is related to the severe suppression of the magnitude of $\mathrm{T}_{\mathrm{B}}(\mathrm{EMR})$ by the applied field $\mathrm{H}_{\mathrm{r}} \simeq 3000$ Oe needed to observe the resonance. Such a suppression of $T_{B}(M)$ was reported earlier in Fig. 5.4 where $T_{B}(M) \simeq 0$ $\mathrm{K}$ for $\mathrm{H}=3200$ Oe was observed and reported in our recent paper [Singh et al, 2008].

In summary, the important new result from the EMR studies is that the temperature dependence of the EMR parameters for the line from majority of the particles in the $D=3.8 \mathrm{~nm}$ sample are consistent with the theoretical predictions of the temperature dependence of the anisotropy shown in Fig. 6.2. Analysis of the temperature dependence of $\mathrm{I}, \mathrm{H}_{\mathrm{r}}$ and $\Delta \mathrm{H}$ for the samples with $\mathrm{D}=11.7,15,21$ and $23 \mathrm{~nm}$ are shown in Fig. 6.11, 6.12, 6.13 and 6.14 respectively. Although $\mathrm{T}_{\mathrm{B}}(\mathrm{EMR})$ can be similarly located in each case, the experimental ratio of $T_{B}(E M R) / T_{B}(M)$ is even smaller as size increases. This is possibly due to the additional complication of the presence of interparticle interaction with increase in size deduced from the analysis of the AC susceptibility measurements in chapter 5. For these reasons, additional analysis on these particles is not presented except to note that the experimental temperature dependence of the EMR parameters becomes weaker with increase in size. This is consistent with the weaker 
temperature dependence of the anisotropy with increase in particle size predicted by the plots of Fig. 6.2.

\subsection{Temperature dependence of Line 1}

Line 1 as indicated in Fig. 6.4 appears in the EMR signals for particle sizes of $\mathrm{D}=3.8,11.7$ and $15 \mathrm{~nm}$ with a hint of the line in the larger size. A similar line is observed in the $\mathrm{SiO}_{2}$ sample prepared without $\mathrm{Ni}$ doping and annealed at $\mathrm{T}=400^{\circ}$ and $800^{\circ} \mathrm{C}$. Temperature variation of $\mathrm{SiO}_{2}$ annealed at $400^{\circ} \mathrm{C}$ was studied in detail from $\mathrm{T}=5$ to 300 $\mathrm{K}$ (see Fig. 6.15). This data was compared with line 1 obtained from $\mathrm{D}=3.8 \mathrm{~nm}$ sample at $\mathrm{T}=5 \mathrm{~K}$. The intensity shows a Curie law variation of the line in undoped $\mathrm{SiO}_{2}$, and in the $3.8 \mathrm{~nm}$ sample in Fig. 6.16. The g value is also the same for both the samples and does not change with temperature as indicated by $\mathrm{H}_{\mathrm{r}}$. However $\Delta \mathrm{H}$ is greater for the $\mathrm{D}=3.8 \mathrm{~nm}$ sample than that for the undoped $\mathrm{SiO}_{2}$ sample.

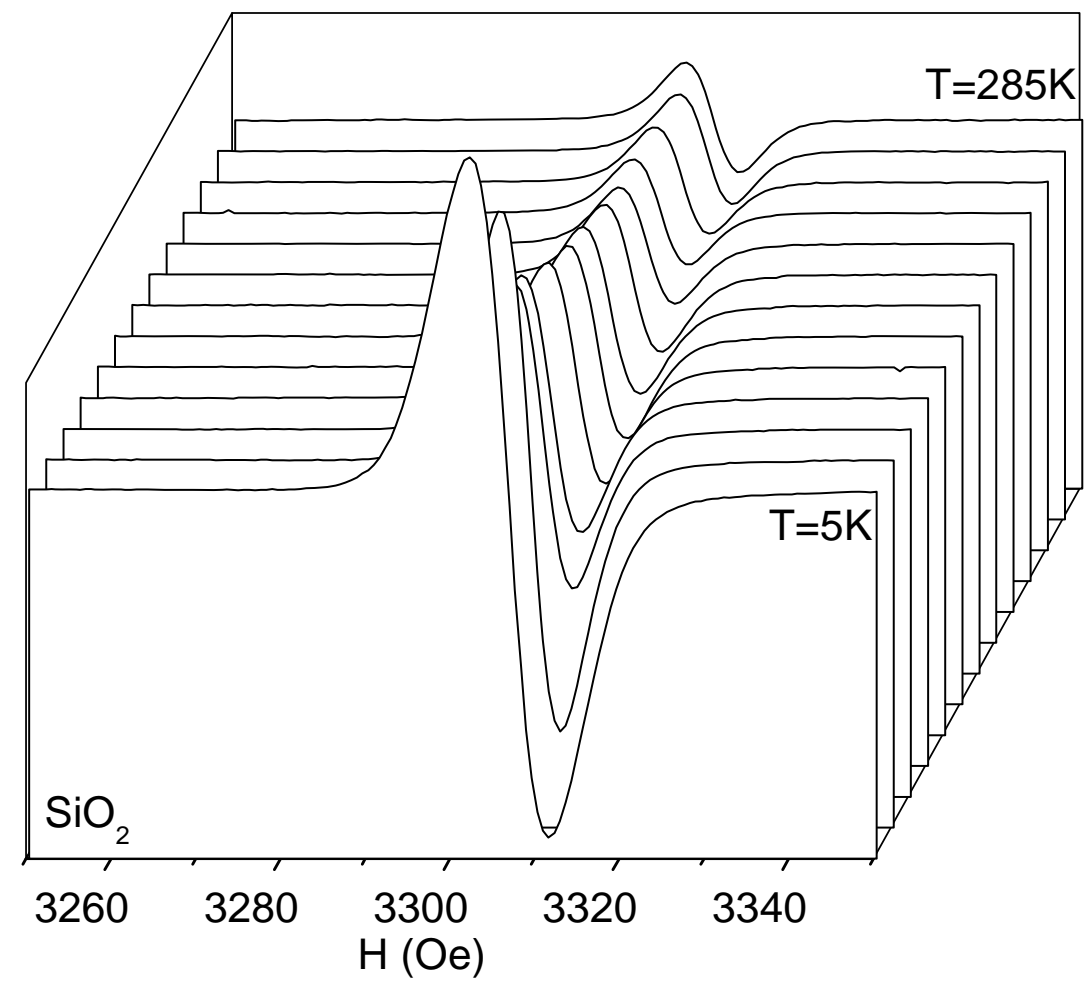

Fig. 6.15 EMR lines for undoped $\mathrm{SiO}_{2}$ for temperatures $\mathrm{T}=5,20,40$, $56,70,83,100,120,155,195,235,265$ and $285 \mathrm{~K}$. 


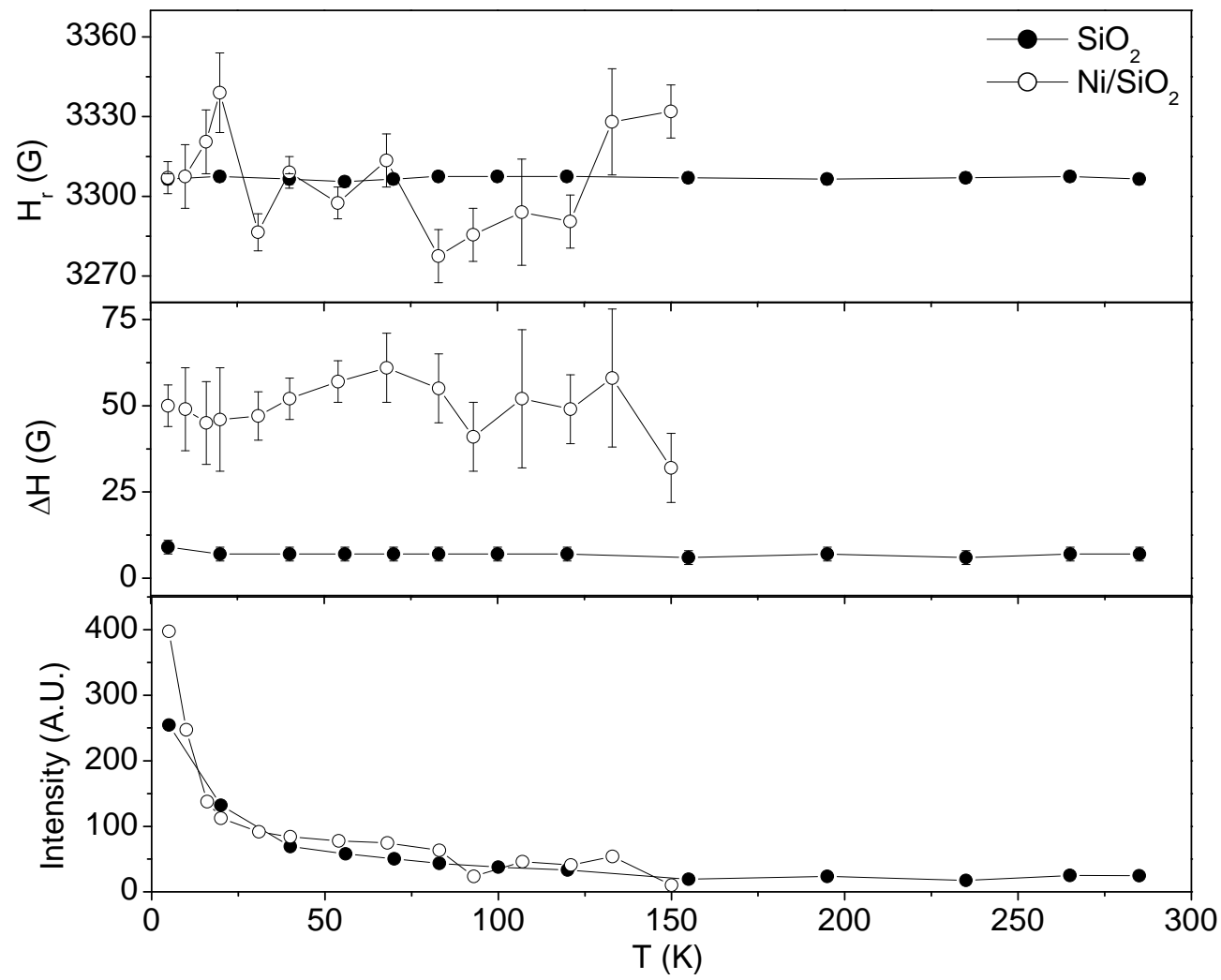

Fig. 6.16 Comparison of $\mathrm{H}_{\mathrm{r}}, \Delta \mathrm{H}$ and $\mathrm{I}$ for $\mathrm{SiO}_{2}$ and $\mathrm{Ni} / \mathrm{SiO}_{2}\left(\mathrm{~T}_{\mathrm{a}}=400^{\circ} \mathrm{C}\right)$.

As it can be seen from the EMR plots, the intensity of the EMR line 1 decreases as the size of the particle increases. In fact, line 1 is extremely weak for the $\mathrm{D}=21 \mathrm{~nm}$ and $\mathrm{D}=23 \mathrm{~nm}$ samples. Recall that to obtain the larger sizes of Ni NPs, the samples were annealed at the higher temperature of $700^{\circ}$ and $800^{\circ} \mathrm{C}$ for the $\mathrm{D}=21 \mathrm{~nm}$ and $\mathrm{D}=23 \mathrm{~nm}$ samples respectively. For the $\mathrm{D}=3.8 \mathrm{~nm}$, annealing was done at $400^{\circ} \mathrm{C}$. To determine whether this higher temperature annealing could explain the decreasing intensity of line 1 in the larger sizes, an undoped $\mathrm{SiO}_{2}$ sample was annealed at $800^{\circ} \mathrm{C}$ followed by EMR measurements at $285 \mathrm{~K}$. As shown in Fig. 6.17, the intensity of the EMR line 1 in the $800^{\circ} \mathrm{C}$ annealed sample is about two order of magnitude weaker than that of the $400^{\circ} \mathrm{C}$ annealed sample. These observations explain that the higher annealing temperature quenches the defect responsible for the EMR signal of line 1. No further studies on the nature of this EMR active defect were carried out since there is already an extensive literature on this matter [Devine, 1988]. It is possible that the larger $\Delta \mathrm{H}$ of this line in the 
$\mathrm{Ni} / \mathrm{SiO}_{2}$ sample may be due to the inhomogeneous field produced by the doped $\mathrm{Ni}$ atoms surrounding the $\mathrm{SiO}_{2}$ defect.

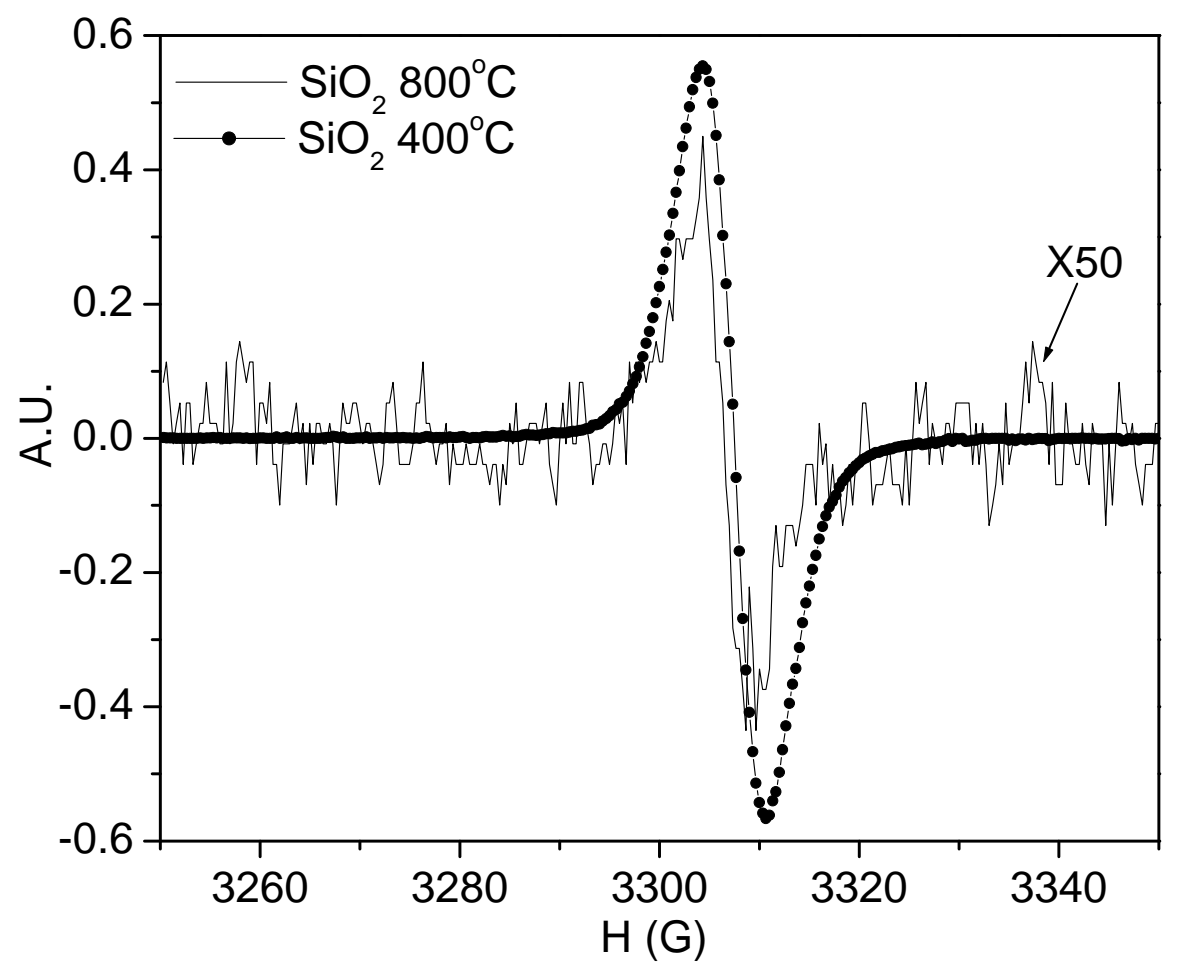

Fig. 6.17 Comparison of $\mathrm{SiO}_{2}\left(\mathrm{~T}_{\mathrm{a}}=400^{\circ}\right.$ and $\left.800^{\circ} \mathrm{C}\right)$ signals at room temperature.

The EMR lines for the $\mathrm{SiO}_{2}$ at $\mathrm{T}_{\mathrm{a}}=400^{\circ} \mathrm{C}$ and $\mathrm{D}=3.8 \mathrm{~nm}$ at $\mathrm{T}=5 \mathrm{~K}$ were fit to the derivative of the Lorenzian and Gaussian lines in Fig. 6.18 and 6.19 as described by [Poole, 1983]:

$$
\begin{aligned}
L^{\prime}(H) & =\frac{16 y_{m}\left[\left(H-H_{r}\right) /\left(0.5 \Delta H_{p p}\right)\right]}{\left\{3+\left[\left(H-H_{r}\right) /\left(0.5 \Delta H_{p p}\right)\right]^{2}\right\}^{2}} \\
G^{\prime}(H) & =y_{m} \cdot\left(\frac{H-H_{r}}{0.5 \Delta H_{p p}}\right) \exp \left\{-\frac{1}{2}\left[\left(\frac{H-H_{r}}{0.5 \Delta H_{p p}}\right)^{2}-1\right]\right\}
\end{aligned}
$$

where $\mathrm{H}_{\mathrm{r}}$ is the resonance field, $\Delta \mathrm{H}_{\mathrm{pp}}$ is the width between maximum and minimum of the peak, and $y_{m}{ }^{\prime}$ is half the amplitude of the maximum and minimum of the peak of the derivative of the Lorenzian and the Gaussian. The EMR spectra fits neither of the two line shapes exactly but is in the middle of the two spectra. This deviation of the EMR spectra from the exact Lorenzian or Gaussian lineshape was also observed by [Deigen et 
al, 1970] where the recorded spectra of $\mathrm{Fe}^{3+}$ in $\mathrm{Al}_{2} \mathrm{O}_{3}$ was found to be in between Gaussian and Lorenzian in shape and a transformation from Lorenzian to Gaussian was observed by [Swarup, 1959] where $\mathrm{Cr}^{3+}$ concentration was changed in potassium cobalticyanide and potassium aluminum alum single crystals.

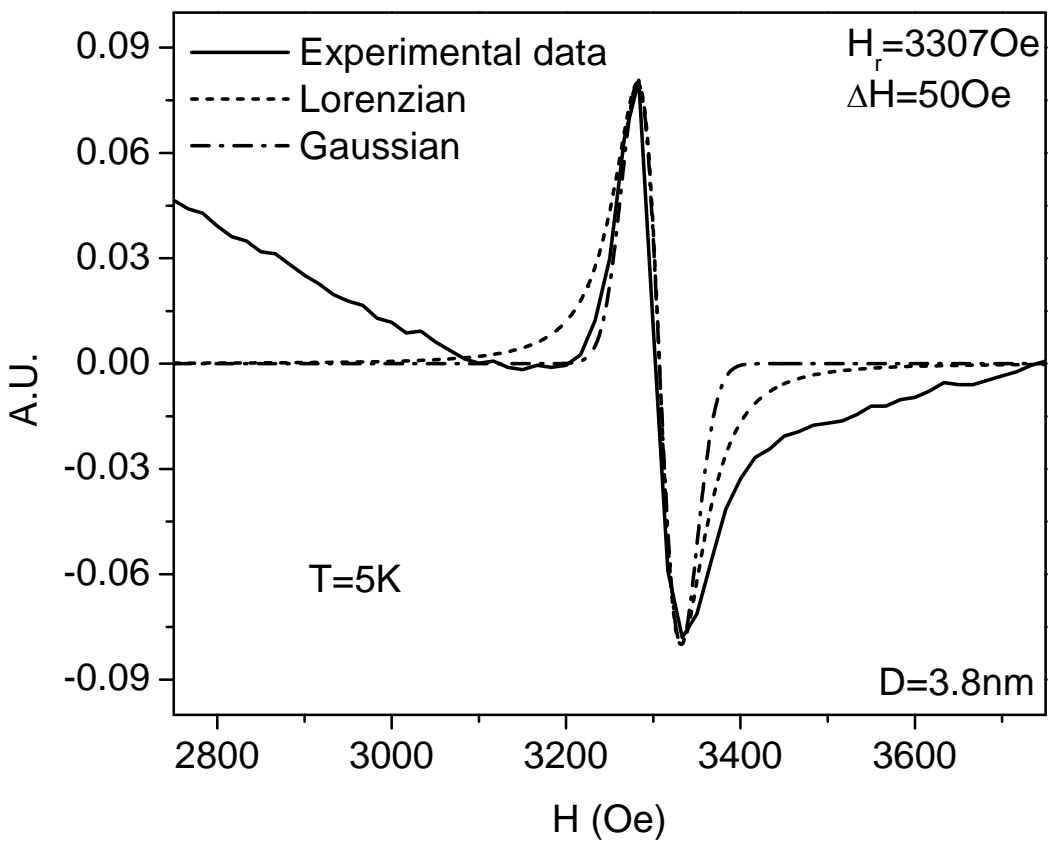

Fig.6.18 Gaussian and Lorenzian fits to experimental data for $\mathrm{D}=3.8 \mathrm{~nm}$.

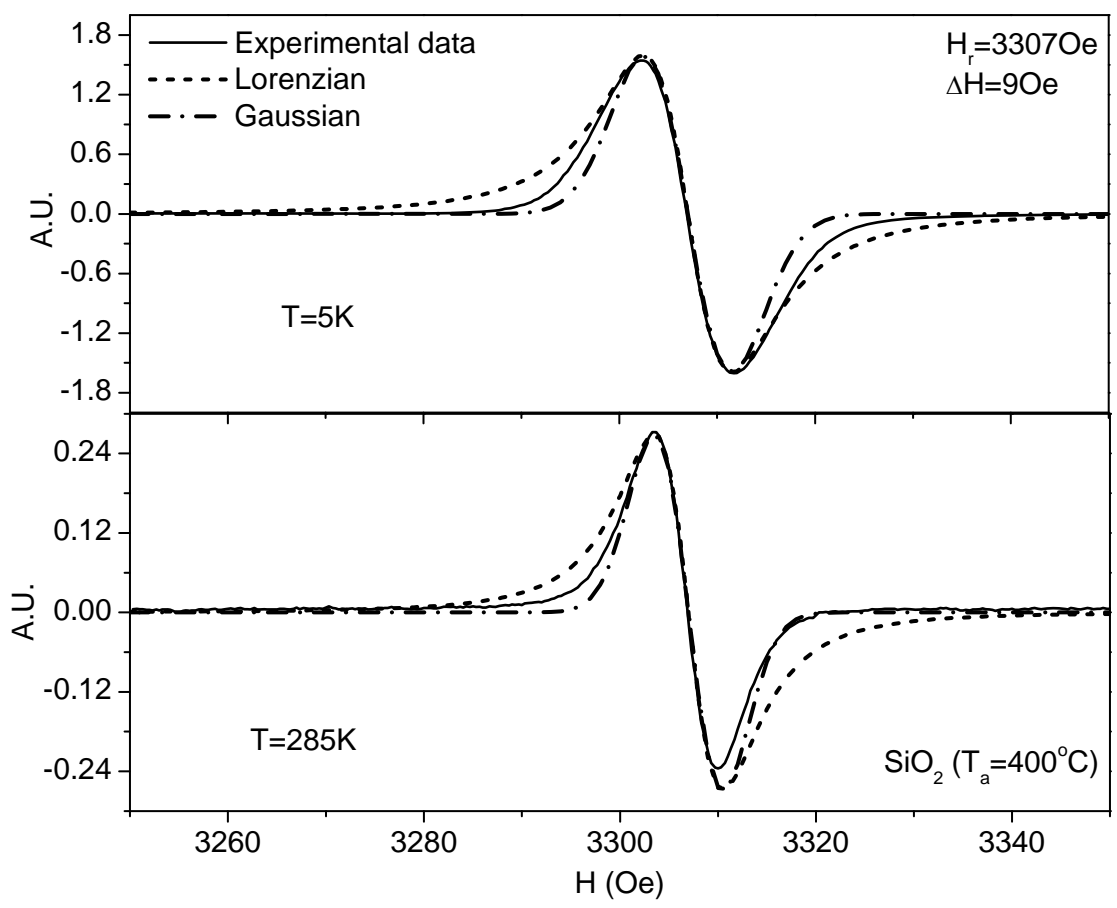

Fig.6.19 Gaussian and Lorenzian fits to experimental data for $\mathrm{SiO}_{2}$. 


\section{CHAPTER VII}

\section{Summary and Conclusions}

In this thesis, the results of a detailed study of the structural and magnetic properties of Ni NPs using x-ray diffraction, SQUID magnetometry and electron magnetic resonance are presented. The primary results of the above studies are described below.

Nanoparticles of Ni embedded in amorphous silica matrix without any oxidation to $\mathrm{NiO}$ were prepared successfully with different particle sizes and in different concentrations. XRD and TEM techniques were used to investigate the structure of the $\mathrm{Ni}$ NPs with $15 \% \mathrm{Ni} / 85 \% \mathrm{SiO}_{2}$ concentrations. The particle sizes as obtained by TEM using $\log$-normal fits were with $\mathrm{D}=3.8,11.7,15,21$ and $23 \mathrm{~nm}$. These particles were nearly spherical in shape as characterized by TEM. XRD measurements showed that the powder samples were small in crystalline size and the particle size obtained from XRD and TEM were comparable.

Detailed studies of the behavior of the magnetization (M) as a function of temperature $(\mathrm{T})(\mathrm{T}=2$ to $300 \mathrm{~K})$ and magnetic fields $\mathrm{H}$ upto $\pm 65 \mathrm{kOe}$ are reported to investigate the size dependence of these properties. Blocking temperature $\left(T_{B}\right)$, determined from the bifurcation of the zero field cooled and field cooled $\mathrm{M}$ vs. T plots was found to increase with increase in size $\mathrm{D}$ as expected from theory. Variation of $\mathrm{T}_{B}$ with applied field $\mathrm{H}$ was investigated in detail for the $\mathrm{D}=3.8 \mathrm{~nm}$ sample. Following theory, the data was found to fit the equation $T_{B}(H)=33\left[1-\left(H / H_{0}\right)\right]^{m}$ with $m=2$ for lower fields and $m=3 / 2$ for higher fields and with $\mathrm{H}_{0}=3200$ Oe.

For $\mathrm{T}>\mathrm{T}_{\mathrm{B}}$, the plots of $\mathrm{M}$ vs. $\mathrm{H}$ were fit to the Langevin variation expected for superparamagnetism to determine $\mu_{\mathrm{P}}$, the magnetic moment per particle. The fits yielded $\mu_{\mathrm{P}}=3500,7500,11000,17000$ and $25000 \mu_{\mathrm{B}}$ for the sizes $\mathrm{D}=3.8,11.7,15,21$ and $23 \mathrm{~nm}$. Although the increase in $\mu_{\mathrm{P}}$ with size $\mathrm{D}$ was understood qualitatively because the presence of larger number of atoms with increasing size, a satisfactory quantitative fit was not obtained. Temperature variation of the coercivity $\mathrm{H}_{\mathrm{C}}$ was investigated and it was found to decrease as $T_{B}$ is approached from the lower temperature. However $\mathrm{H}_{C}$ does not become zero at $T_{B}$ as expected from theory, possibly because of the presence of 
unblocked larger particles resulting from the size distribution. Any presence of exchange bias for a field cooled sample to $\mathrm{T}<\mathrm{T}_{\mathrm{B}}$ was carefully checked for $\mathrm{D}=3.8 \mathrm{~nm}$ sample with negative results. This confirmed the absence of $\mathrm{NiO}$ consistent with the XRD measurements.

Temperature variations of the AC susceptibility $\chi^{\prime}$ and $\chi^{\prime \prime}$ were carried using the SQUID magnetometer at the measuring frequencies $\mathrm{f}_{\mathrm{m}}=0.1,1,99,499$ and $997 \mathrm{~Hz}$. The blocking temperature $T_{B}$ as determined from the peaks in the $\chi^{\prime \prime}$ vs. $T$ data were first fit to the Néel-Arrhenius relation $f_{m}=f_{o} \exp \left(-T_{a} / T_{B}\right)$ and then to the Vogel Fulcher relation $f_{m}=f_{0} \exp \left(-T_{a} /\left(T_{B}-T_{0}\right)\right)$ to determine the quantities $f_{0}$ (attempt frequency), $T_{a}($ effective anisotropy temperature) and $\mathrm{T}_{\mathrm{o}}$ representing any interparticle interaction. From a careful analysis of the data, $\mathrm{f}_{\mathrm{o}}=1.82 \times 10^{10} \mathrm{~Hz}$ was derived for this system with $\mathrm{T}_{\mathrm{o}}=0,0,6.6$ and $12.5 \mathrm{~K}$ for the $\mathrm{D}=3.8,11.7,15,21$, and $23 \mathrm{~nm}$ particles. As expected, $\mathrm{T}_{\mathrm{a}}$ increased with increase in D from which the variation of the effective anisotropy constant with size was determined. The absence of the interpaticle interaction (represented by $T_{0}$ ) for smaller particles and its weak presence for larger particles was verified by calculating the magnitude of the quantity $\Phi=\Delta \mathrm{T}_{\mathrm{B}} /\left[\mathrm{T}_{\mathrm{B}} \Delta \log _{10} \mathrm{f}_{\mathrm{m}}\right]$ for the four particle sizes. Finally, the theoretical relationship between $\chi^{\prime}$ and $\chi^{\prime \prime}$ given $\chi^{\prime \prime}=\mathrm{C} \partial\left(\chi^{\prime} \mathrm{T}\right) / \partial \mathrm{T}$ was verified by computing $\partial\left(\chi^{\prime} \mathrm{T}\right) / \partial \mathrm{T}$ using the experimental $\chi^{\prime}$ vs. T data and comparing it with the experimental $\chi^{\prime \prime}$ vs. T data.

Additional computational studies were done by computing $\chi^{\prime}$ vs. T and $\chi^{\prime \prime}$ vs. $T$ from the theoretical equations for non-interacting particles using the parameters $f_{0}, T_{a}$ and $\mathrm{T}_{\mathrm{B}}$ determined above and fixed size $\mathrm{D}$. Although the forms of the computed $\chi^{\prime} \mathrm{vs.} \mathrm{T}$ and $\chi^{\prime \prime}$ vs. $T$ are somewhat similar to the ones obtained experimentally, the widths of the experimental curves are considerably larger. This is expected since the rather wide size distribution of experimental particle sizes were neglected in the computations. Computations including size distributions were found to be difficult to do.

Temperature variations of the electron magnetic resonance (EMR) spectra at $\mathrm{f}_{\mathrm{m}}=9.28 \mathrm{GHz}$ were investigated for the $\mathrm{D}=3.8,11.7,15,21$ and $23 \mathrm{~nm}$ samples. In general three lines were observed with strong temperature variations of their EMR parameters e.g. the resonance field $\mathrm{H}_{\mathrm{r}}$, peak to peak linewidth $\Delta \mathrm{H}$ and the line intensity I. To understand the origin of these lines, theoretical variation of the anisotropy for the cubic 
symmetry of $\mathrm{Ni}$ with change in temperature and particle size was investigated and compared with the experimental variations. Narrow line 1 with temperature independent $\mathrm{H}_{\mathrm{r}}$ and $\Delta \mathrm{H}$ and Curie law variation of its intensity was identified with the EMR active defects in the $\mathrm{SiO}_{2}$ matrix. The dominant line 2 whose linewidth and lineshift and line intensity increase with decreasing temperature as approach to $T_{B}$ is associated with majority of the Ni particles. Finally weak line 3 with larger shifts in $\mathrm{H}_{\mathrm{r}}$ is associated with larger particles or cluster of $\mathrm{Ni}$ particles. Identification of these lines based on the expected temperature and size dependence of the anisotropy is considered to be an important contribution to EMR studies. 


\section{REFERENCES}

1. Aharoni, A., Phys. Rev. B 7, 1103 (1973).

2. Andersson, J. O., Djurberg, C., Jonsson, T., Svedlindh, P. and Nordblad, P., Phys. Rev. B 56, 13983 (1997).

3. Biasi, S. de, Ronaldo and Devezas, C. Tessaleno, J. Appl. Phys. 49, 2466 (1978).

4. Bodkar, F., Morup, S. and Linderoth, S., Phys. Rev. Lett. 72, 282 (1994).

5. Brown, W. F. Jr., J. Appl. Phys. 34, 1319 (1963).

6. Chikazumi, Sóshin, "Physics of Ferromagnetism", (Oxford University Press, 1997).

7. Chinnasamy, C. N., Jeyadevan, B., Shinoda, K., Tohji, K., Narayanasamy, A., Sato, K. and Hisano, S., J. Appl. Phys. 97, 10J309-1 (2005).

8. Chudnovsky, Eugene M. and Tejada, Javier, "Magnetic Quantum Tunneling of the Magnetic moment", (Cambridge University Press, 1998).

9. Crystallography Lab, "Characteristic radiation", The University of Oklahoma, http://xrayweb.chem.ou.edu/notes/xray.html, (2007).

10. Cullity, B. D., "Introduction to Magnetic Materials", (Addison-Wesley Publishing Company, 1972).

11. Deigen, M. F., Geifman, I. N., Glinchyk, M. D., Fiz. Tverd. Tela 12, 1469 (1970).

12. Design, Quantum, “MPMS MultiVu Application User’s Manual”, (1999).

13. Devine, R. A. B., "The Physics and Technology of Amorphous $\mathrm{SiO}_{2}$ ", (Plenum Press, 1988).

14. Djurberg, C., Svedlindh, P., Nordblad, P ., Hansen, M. F., Bødker, F. and Mørup, S., Phys. Rev. Lett. 79, 5414 (1997).

15. Dormann, J. L., Bessais L., and Fiorani, D., J. Phys. C: Solid State Phys. 21, 2015 (1988).

16. Estournès, C., Lutz, T., Happich, J., Quaranta, T., Wissler, P. and Giulle, J. L., J. Magn. Magn. Mater. 173, 83 (1997).

17. Fonseca, F. C., Goya, G. F., Jardim, R. F., Carreño, N. L. V., Longo, E., Leite, E. R. and Muccillo, R., Appl. Phys. A 76, 621 (2002). 
18. Fonseca, F. C., Goya, G. F., Jardim, R. F., Muccillo, R., Carreño, N. L. V., Longo, E. and Leite, E. R., Phys. Rev. B 66, 104406 (2002).

19. Gilmore, K., Idzerda, Y. U., Klem, M. T., Allen, M., Douglas, T. and Young, M., J. Appl. Phys. 97, 10B301 (2005).

20. Gittleman, J. I., Abeles, B. and Bozowski, S., Phys. Rev. B 9, 3891 (1974).

21. Goya, G. F., Fonseca, F. C. R. F., Jardim, R. F., Muccillo, R., Carreño, N. L. V., Longo, E. and Leite, E. R., J. Appl. Phys. 93, 6531 (2003).

22. Hou, Y., Kondoh, H., Ohta, T. and Gao, S., Applied Surface Science 241, 218-222 (2005).

23. Ibrahim, Manjula M., Zhao, Jianmin, and Seehra, Mohindar S., J. Mater. Res. 7, 1856 (1992).

24. Kliava, Janis and Berger René, J. Magn. Magn. Mater. 205, 328-342 (1999).

25. Kittel, Charles, "Introduction to Solid State Physics", (John Wiley \& Sons Inc., 1996).

26. Klug, Harold P. and Alexander Leroy E., "X-ray Diffraction Procedures", (A Wiley-Interscience Publication, 1954).

27. Konchits, A. A., Motsnyi, F. V., Petrov, Yu. N., Kolesnik, S. P., Yefanov, V. S., Terranova, M. L., Tamburri, E., Orlanducci, S., Sessa, V. and Rossi, M., J. Appl. Phys. 100, 124315 (2006).

28. Kuiper, Pieter, "Levels involved in $\mathrm{Cu} \mathrm{K}$ x-ray emission", http://en.wikipedia.org/wiki/Image:Copper K Rontgen.png, (2006).

29. Leite, E. R., Carreño, N. L. V., Longo, E., and Pontes, F. M., Chem. Mater. 14, 3722 (2002).

30. Leite, E. R., Carreño, N. L. V., Longo, E., Valentini, A. and Probst, L. F. D., J. Nanosci. Nanotech. 2, 89 (2002).

31. Luis, F., Petroff, F., Torres, J. M., García, L. M., Bartolomé, J., Carrey, J. and Vaurès, A., Phys. Rev. Lett. 88, 217205-1 (2002).

32. Lundgren, L., Svedlindh, P. and Beckman, O., J. Magn. Magn. Mater. 25, 33-38 (1981). 
33. Lutz, T., Estournès, C. and Giulle, J. L., J. Sol-Gel Sci. and Tech. 13, 929 (1998).

34. Morrish, Allan H., "The Physical Principles of Magnetism”, (IEEE Press, 2001).

35. Néel, L., Ann. Géophys (C.N.R.S) $\underline{5}, 99$ (1949).

36. Nogués, J. and Schuller, Ivan K., J. Magn. Magn. Mater. 192, 203-232 (1999).

37. Pal, S., Dutta, P., Shah, N., Huffman, G. P. and Seehra, M. S., IEEE Tranactions on Magnetics 43, 3091 (2007).

38. Petracic, O., Glatz, A. and Kleemann, W., Phys. Rev. B 70, 214432 (2004).

39. Poole, Charles P. Jr., "Electron Spin Resonance: A Comprehensive Treatise on Experimental Techniques", (John Wiley \& Sons Inc., 1983).

40. Poole, Charles P. Jr. and Owens, Frank J., "Introduction to Nanotechnology", (John Wiley \& Sons Inc., 2003).

41. Raikher, Yuri L. and Stepanov, Victor I., Phys. Rev. B 50, 6250 (1994).

42. Roy, Aparna, Srinivas, V., Ram, S., Toro, J. A. De and Mizutani, U., Phys. Rev. B 71,184443 (2005).

43. Sahoo, Y., He, Y., Swihart, M. T., Wang, S., Luo, H., Furlani, E. P. and Prasad, P. N., J. Appl. Phys. 98, $0 J 54308$ (2005).

44. Seehra, M. S., Dutta, P., Shim, H. and Manivannan, A., Solid State Commun. 129, 721-725 (2004).

45. Sharma, V. K. and Baiker, A., J. Chem. Phys. 75, 5596 (1981).

46. Shim, H., Dutta, P., Seehra, M. S. and Bonevich, J., Solid St. Commun. 145, 192 (2008).

47. Shim, H., Mannivannan, A., Seehra, M. S., Reddy, K. M. and Punnoose, A., J. Appl. Phys. 99, 08Q503 (2006).

48. Singh, V., Seehra, M. S. and Bonevich, J., J. Appl. Phys. 103, 07D524 (2008).

49. Swarup, P., Can. J. Phys. 37, 848 (1959).

50. Victora, R. H., Phys. Rev. Lett. 63, 457 (1989).

51. Walton, D., Phys. Rev. Lett. 65, 1170 (1990). 
52. Williamson, G. K. and Hall, W. H., Acta Metall. 1, 22 (1953).

53. Winter, Mark, "The Periodic table on the WWW", The University of Sheffield and WebElements, http://www.webelements.com/nickel/, (2007).

54. Yanes, R., Fesenko, O. C., Kachkachi, H., Garanin, D. A., Evans, R. and Chantrell, R. W., Phys. Rev. B 76, 064416 (2007).

55. Zeng, H., Skomski, R., Menon, L., Liu, Y., Bandyopadhyay, S. and Sellmyer, D. J., Phys. Rev. B 65, 134426 (2002). 


\section{APPENDIX I \\ Calculations for Nickel Nanoparticles embedded in Silica matrix}

In this appendix calculations for 1,5 and $15 \% \mathrm{Ni} / \mathrm{SiO}_{2}$ are done following the paper by [Leite et al, 2002] in order to prepare Ni NPs embedded in $\mathrm{SiO}_{2}$ matrix. Details of the calculations are shown below for the 3 samples.

\section{$\underline{15 \% \mathrm{Ni} / \mathrm{SiO}_{2}} \underline{(14.8 / 85.2)}$}

Nickel Nitrate: $\mathrm{Ni}\left(\mathrm{NO}_{3}\right)_{2} \bullet 6 \mathrm{H}_{2} \mathrm{O}$, Molecular Weight $(\mathrm{MW})=290.83$, Mass $(\mathrm{m})=1 \mathrm{gm}$ TEOS: $\left(\mathrm{C}_{2} \mathrm{H}_{5}\right)_{4} \mathrm{SiO}_{4}(\mathrm{MW})=208.33, \mathrm{~m}=4 \mathrm{gm}$

Citric Acid: $\mathrm{C}_{6} \mathrm{H}_{8} \mathrm{O}_{7}(\mathrm{MW})=192.13, \mathrm{~m}=11.53 \mathrm{gm}$

Ethylene Glycol: $\mathrm{C}_{2} \mathrm{H}_{6} \mathrm{O}_{2}(\mathrm{MW})=62.07, \mathrm{~m}=7.69 \mathrm{gm}$

Mass of Silica $=\left(\mathrm{SiO}_{2} /\left(\mathrm{C}_{2} \mathrm{H}_{5}\right)_{4} \mathrm{SiO}_{4}\right) \times \mathrm{m}=((28.09+2(16)) / 208.33) \times 4 \mathrm{gm}=1.15 \mathrm{gm}$

Mass of Nickel $=\left(\mathrm{Ni} / \mathrm{Ni}\left(\mathrm{NO}_{3}\right)_{2} \bullet 6 \mathrm{H}_{2} \mathrm{O}\right) \times \mathrm{m}=(58.7 / 290.83) \times 1 \mathrm{gm}=0.20 \mathrm{gm}$

So we have $0.20 \mathrm{gm}$ of Nickel in $1.15 \mathrm{gm}$ of $\mathrm{SiO}_{2}$.

Total mass: $(1.15+0.20)=1.35 \mathrm{gm}$

$\%$ Nickel $=(0.20 / 1.35) * 100=14.81 \%$

$\%$ Silica $=(1.15 / 1.35) * 100=85.19 \%$

The final nanocomposite was $\left(\mathrm{Ni} / \mathrm{SiO}_{2}\right)$ with composition (14.81/85.19).

Moles of $\mathrm{Ni}\left(\mathrm{NO}_{3}\right)_{2} \bullet 6 \mathrm{H}_{2} \mathrm{O}=$ mass $/$ molar mass $=1 / 290.83=0.00344 \mathrm{~mol}$

Moles of TEOS $=4 / 208.33=0.01920 \mathrm{~mol}$

Moles of Citric Acid $=11.53 / 192.13=0.06001 \mathrm{~mol}$

Total moles $=0.00344+0.01920=0.02264 \mathrm{~mol}$

Citric Acid to metal( $\mathrm{Ni}+\mathrm{Si})$ ratio was $(2.65: 1)$ or $(0.06001: 0.02264)$

Ethylene Glycol : citrate solution in mass ratio (40.01:59.99) which was $(40.01 / 59.99) \times 11.53 \mathrm{gm}=7.69 \mathrm{gm}$ 
$\underline{1 \% \mathrm{Ni} / \mathrm{SiO}_{2}} \underline{(0.7 / 99.3)}$

Nickel Nitrate: $\mathrm{Ni}\left(\mathrm{NO}_{3}\right)_{2} \bullet 6 \mathrm{H}_{2} \mathrm{O}$, Molecular Weight $(\mathrm{MW})=290.83$, Mass $(\mathrm{m})=0.05 \mathrm{gm}$ TEOS: $\left(\mathrm{C}_{2} \mathrm{H}_{5}\right)_{4} \mathrm{SiO}_{4}(\mathrm{MW})=208.33, \mathrm{~m}=4.95 \mathrm{gm}$

Citric Acid: $\mathrm{C}_{6} \mathrm{H}_{8} \mathrm{O}_{7}(\mathrm{MW})=192.13, \mathrm{~m}=13.63 \mathrm{gm}$

Ethylene Glycol: $\mathrm{C}_{2} \mathrm{H}_{6} \mathrm{O}_{2}(\mathrm{MW})=62.07, \mathrm{~m}=9.19 \mathrm{gm}$

Mass of Silica $=\left(\mathrm{SiO}_{2} /\left(\mathrm{C}_{2} \mathrm{H}_{5}\right)_{4} \mathrm{SiO}_{4}\right) \times \mathrm{m}=((28.09+2(16)) / 208.33) \times 4.95 \mathrm{gm}=1.43 \mathrm{gm}$

Mass of $\mathrm{Nickel}=\left(\mathrm{Ni} / \mathrm{Ni}\left(\mathrm{NO}_{3}\right)_{2} \bullet 6 \mathrm{H}_{2} \mathrm{O}\right) \times \mathrm{m}=(58.7 / 290.83) \times 0.05 \mathrm{gm}=0.01 \mathrm{gm}$

So we have $0.01 \mathrm{gm}$ of Nickel in $1.43 \mathrm{gm}$ of $\mathrm{SiO}_{2}$.

Total mass: $(1.43+0.01)=1.44 \mathrm{gm}$

$\%$ Nickel $=(0.01 / 1.44) * 100=0.7 \%$

$\%$ Silica $=(1.43 / 1.44) * 100=99.3 \%$

The final nanocomposite was $\left(\mathrm{Ni} / \mathrm{SiO}_{2}\right)$ with composition (0.7/99.3).

Moles of $\mathrm{Ni}\left(\mathrm{NO}_{3}\right)_{2} \cdot 6 \mathrm{H}_{2} \mathrm{O}=$ mass $/$ molar mass $=0.05 / 290.83=0.00017 \mathrm{~mol}$

Moles of TEOS $=4.95 / 208.33=0.02376 \mathrm{~mol}$

Moles of Citric Acid = 13.63/192.13=0.07094 mol

Total moles $=0.00017+0.02376=0.02393 \mathrm{~mol}$

Citric Acid to metal(Ni+Si) ratio was $(2.96: 1)$ or $(0.07094: 0.02393)$

Ethylene Glycol : citrate solution in mass ratio (40.27:59.73) which was

$(40.27 / 59.73) \times 13.63 \mathrm{gm}=9.19 \mathrm{gm}$ 


\section{$\underline{5 \% \mathrm{Ni} / \mathrm{SiO}_{2}} \underline{(3.5 / 96.5)}$}

Nickel Nitrate: $\mathrm{Ni}\left(\mathrm{NO}_{3}\right)_{2} \bullet 6 \mathrm{H}_{2} \mathrm{O}$, Molecular Weight $(\mathrm{MW})=290.83$, mass $(\mathrm{m})=0.25 \mathrm{gm}$

TEOS: $\left(\mathrm{C}_{2} \mathrm{H}_{5}\right)_{4} \mathrm{SiO}_{4}(\mathrm{MW})=208.33, \mathrm{~m}=4.75 \mathrm{gm}$

Citric Acid: $\mathrm{C}_{6} \mathrm{H}_{8} \mathrm{O}_{7}(\mathrm{MW})=192.13, \mathrm{~m}=13.63 \mathrm{gm}$

Ethylene Glycol: $\mathrm{C}_{2} \mathrm{H}_{6} \mathrm{O}_{2}(\mathrm{MW})=62.07, \mathrm{~m}=9.09 \mathrm{gm}$

Mass of Silica $=\left(\mathrm{SiO}_{2} /\left(\mathrm{C}_{2} \mathrm{H}_{5}\right)_{4} \mathrm{SiO}_{4}\right) \times \mathrm{m}=((28.09+2(16)) / 208.33) \times 4.75 \mathrm{gm}=1.37 \mathrm{gm}$

Mass of Nickel $=\left(\mathrm{Ni} / \mathrm{Ni}\left(\mathrm{NO}_{3}\right)_{2} \bullet 6 \mathrm{H}_{2} \mathrm{O}\right) \times \mathrm{m}=(58.7 / 290.83) \times 0.25 \mathrm{gm}=0.05 \mathrm{gm}$

So we have $0.05 \mathrm{gm}$ of Nickel in $1.37 \mathrm{gm}$ of $\mathrm{SiO}_{2}$.

Total mass: $(1.37+0.05)=1.42 \mathrm{gm}$

$\%$ Nickel $=(0.05 / 1.42) * 100=3.5 \%$

$\%$ Silica $=(1.37 / 1.42) * 100=96.5 \%$

The final nanocomposite was $\left(\mathrm{Ni} / \mathrm{SiO}_{2}\right)$ with composition (0.7/99.3).

Moles of $\mathrm{Ni}\left(\mathrm{NO}_{3}\right)_{2} \cdot 6 \mathrm{H}_{2} \mathrm{O}=$ mass $/$ molar mass $=0.05 / 290.83=0.00017 \mathrm{~mol}$

Moles of TEOS $=4.95 / 208.33=0.02376 \mathrm{~mol}$

Moles of Citric Acid = 13.63/192.13=0.07094 mol

Total moles $=0.00017+0.02376=0.02393 \mathrm{~mol}$

Citric Acid to metal(Ni+Si) ratio was $(2.96: 1)$ or $(0.07094: 0.02393)$

Ethylene Glycol : citrate solution in mass ratio (40.01:59.99) which was $(40.01 / 59.99) \times 13.63 \mathrm{gm}=9.09 \mathrm{gm}$ 


\section{APPENDIX II}

\section{Derivation for AC Susceptibility $\chi^{\prime}$ and $\chi^{\prime \prime}$}

In this appendix the derivation for the ac susceptibility has been done in detail. The equations for the ac susceptibility have been obtained from the paper by [Andersson et al, 1997]. In this derivation we derive the expression for the in-phase and out-of-phase ac susceptibility of a particle in the presence of a small field with random particle easy axes where we start by considering the relation for energy given by:

$$
E_{i}=-K V_{i}\left(\hat{\mu}_{i} \cdot \hat{n}_{i}\right)^{2}-M_{S} V_{i} \hat{\mu}_{i} \cdot \vec{B}_{i}
$$

Here the first term in the energy comes from the anisotropy that arises from the moment not lying along the direction of the easy magnetization axis. The second term is the Zeeman term that comes from the magnetic moment placed in an external magnetic field. $\hat{\mu}_{i}$ is the unit vector along the direction of the magnetic moment given by $\vec{\mu}_{i}=M_{S} V_{i} \hat{\mu}_{i}$, $\hat{n}_{i}$ is unit vector along the easy magnetization axes, $K$ is the anisotropy constant, $V_{i}$ is the particle volume, $M_{S}$ is the saturation magnetization and $H_{a}$ is the applied field with $\vec{B}_{i}=\mu_{o} H_{a} \hat{z}$.

The figure below gives the direction of the magnetic moment, easy magnetization axis and the externally applied field with respect to one another defined by the angles $\psi_{i}$ between the easy magnetization axis and the external field and the angle $\alpha_{i}$ between the magnetic moment and the easy magnetization axis.

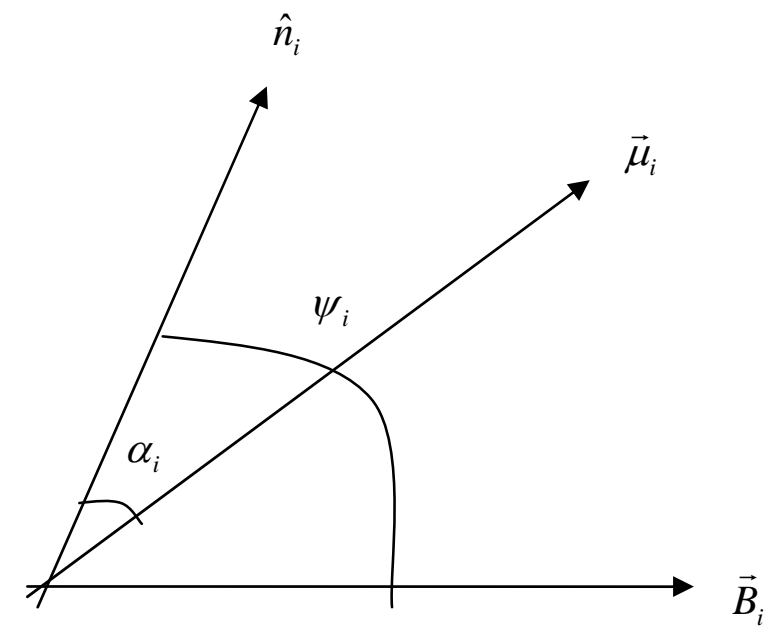


We can rewrite the expression for energy as:

$$
E_{i}=-K V_{i}\left[\cos ^{2}\left(\alpha_{i}\right)+\left(\frac{M_{S}}{K}\right)\left|\vec{B}_{i}\right| \cos \left(\psi_{i}-\alpha_{i}\right)\right]
$$

Minimizing the energy with respect to the angle $\alpha_{i}$ gives:

$$
\frac{d E_{i}}{d \alpha_{i}}=-K V_{i}\left[-2 \cos \left(\alpha_{i}\right) \sin \left(\alpha_{i}\right)+\left(\frac{M_{S}}{K}\right)\left|\vec{B}_{i}\right| \sin \left(\psi_{i}-\alpha_{i}\right)\right]
$$

where in order to obtain the points of inflection that will give the energy minimum we calculate $\frac{d E_{i}}{d \alpha_{i}}=0$ thus obtaining the expression:

$$
\left.2 \cos \left(\alpha_{i}\right) \sin \left(\alpha_{i}\right)=\left(\frac{M_{S}}{K}\right)\left|\vec{B}_{i}\right| \sin \left(\psi_{i}-\alpha_{i}\right)\right]
$$

We can set $x=\frac{M_{S}}{K}\left|\vec{B}_{i}\right|=\frac{M_{S}}{K}\left(\mu_{o} H_{a}\right)$ to obtain:

$$
2 \sin \alpha_{i} \cos \alpha_{i}=x\left[\sin \left(\psi_{i}\right) \cos \left(\alpha_{i}\right)-\cos \left(\psi_{i}\right) \sin \alpha_{i}\right]
$$

\section{CASE I}

Magnetic field is $90^{\circ}$ or $270^{\circ}$ from the easy magnetization axis

A. Magnetic field $90^{\circ}$ from the easy axis

This can be done by setting $\cos \left(\psi_{i}\right)=0$, and considering the fact that the magnetic moment lies close to the easy magnetization axis implying $\sin \left(\alpha_{i}\right)=\alpha_{i}$ and $\cos \left(\alpha_{i}\right)=1$ for small $\alpha_{i}$. For the magnetic field corresponding to $90^{\circ}$ from the easy axis, Eq. (II.5) reduces to $2 \sin \left(\alpha_{i}\right)=x \sin \left(\psi_{i}\right)$ giving $\alpha_{1}=\frac{x}{2} \sin (\psi)$. The corresponding energy is then given by:

$$
E_{1}=-K V\left[\cos ^{2}\left(\left(\frac{x}{2} \sin (\psi)\right)+x \cos \left(\psi-\frac{x}{2} \sin (\psi)\right)\right]\right.
$$

This expression then simplifies to: 


$$
\begin{gathered}
E_{1}=-K V\left[\frac{1}{2}+\frac{1}{2} \cos (x \sin (\psi))+x\left[\cos (\psi) \cos \left(\frac{x}{2} \sin (\psi)\right)+\sin (\psi) \sin \left(\frac{x}{2} \sin (\psi)\right)\right]\right] \\
=-K V\left[\frac{1}{2}+\frac{1}{2} \cos (x \sin (\psi))+x \cos (\psi) \cos \left(\frac{x}{2} \sin (\psi)\right)+x \sin (\psi) \sin \left(\frac{x}{2} \sin (\psi)\right)\right] \\
=-K V\left[\frac{1}{2}+\frac{1}{2}+x \cos (\psi)+x \sin (\psi) \frac{x}{2} \sin (\psi)\right] \\
E_{1}=-K V[1+x \cos (\psi)]
\end{gathered}
$$

B. Magnetic field $270^{\circ}$ from the easy axis

For the magnetic field corresponding to $270^{\circ}$ from the easy axis the angle $\alpha_{2}=\pi-\frac{x}{2} \sin (\psi)$. The corresponding energy is then given by:

$$
\begin{gathered}
E_{2}=-K V\left[\cos ^{2}\left(\pi-\frac{x}{2} \sin (\psi)\right)+x \cos \left(\psi-\left(\pi-\frac{x}{2} \sin (\psi)\right)\right)\right] \\
=-K V\left[\frac{1}{2}+\frac{1}{2} \cos (2 \pi-x \sin (\psi))+x\left[\cos (\psi) \cos \left(\pi-\frac{x}{2} \sin (\psi)\right)+\sin (\psi) \sin \left(\pi-\frac{x}{2} \sin (\psi)\right)\right]\right] \\
=-K V\left[\frac{1}{2}+\frac{1}{2} \cos (2 \pi-x \sin (\psi))+x\left[-\cos (\psi) \cos \left(\frac{x}{2} \sin (\psi)\right)+\sin (\psi) \sin \left(\frac{x}{2} \sin (\psi)\right)\right]\right. \\
=-K V\left[\frac{1}{2}+\frac{1}{2} \cos (x \sin (\psi))-x \cos (\psi) \cos \left(\frac{x}{2} \sin (\psi)\right)+x \sin (\psi) \sin \left(\frac{x}{2} \sin (\psi)\right)\right] \\
=-K V\left[\frac{1}{2}+\frac{1}{2}-x \cos (\psi)+x \sin (\psi) \frac{x}{2} \sin (\psi)\right] \\
E_{2}=-K V[1-x \cos (\psi)]
\end{gathered}
$$

CASE II

Magnetic field is $0^{\circ}$ or $180^{\circ}$ from the easy magnetization axis

By setting $\sin \left(\psi_{i}\right)=0$, which means that the magnetic field is $0^{\circ}$ or $180^{\circ}$ from the easy magnetization axis, and considering the fact that the magnetic moment lies close to the easy magnetization axis implying $\sin \left(\alpha_{i}\right)=\alpha_{i}$ and $\cos \left(\alpha_{i}\right)=1$ for small $\alpha_{i}$. Eq. (II.5) reduces to $2 \cos \left(\alpha_{i}\right)=-x \cos \left(\psi_{i}\right)$ from where we can obtain $\alpha_{s}$. The next few lines show the calculation for $\alpha_{s}$. 


$$
\begin{aligned}
& 2 \sin \left(\frac{\pi}{2}-\alpha_{i}\right)=-x \cos \left(\psi_{i}\right) \\
& 2\left(\frac{\pi}{2}-\alpha_{i}\right)=-x \cos \left(\psi_{i}\right) \\
& \alpha_{S}=\frac{\pi+x \cos (\psi)}{2}
\end{aligned}
$$

The corresponding energy is then given by:

$$
\begin{gathered}
E_{S}=-K V\left[\cos ^{2}\left(\frac{\pi}{2}+\frac{x}{2} \cos (\psi)\right)+x \cos \left(\psi-\left(\frac{\pi}{2}+\frac{x}{2} \cos (\psi)\right)\right)\right] \\
=-K V\left[\frac{1}{2}+\frac{1}{2} \cos (\pi+x \cos (\psi))+x\left[\cos (\psi) \cos \left(\frac{\pi}{2}+\frac{x}{2} \cos (\psi)\right)+\sin (\psi) \sin \left(\frac{\pi}{2}+\frac{x}{2} \cos (\psi)\right)\right]\right] \\
=-K V\left[\frac{1}{2}-\frac{1}{2} \cos (x \cos (\psi))-x \cos (\psi) \sin \left(\frac{x}{2} \cos (\psi)\right)+x \sin (\psi) \cos \left(\frac{x}{2} \cos (\psi)\right)\right] \\
E_{S}=-K V\left[\frac{1}{2}-\frac{1}{2}+x \cos (\psi) \frac{x}{2} \cos (\psi)+x \sin (\psi)\right]
\end{gathered}
$$


The following diagrams show the possible configurations and the energy values calculated for the corresponding angles from $E_{1}, E_{2}$ and $E_{s}$ relations.
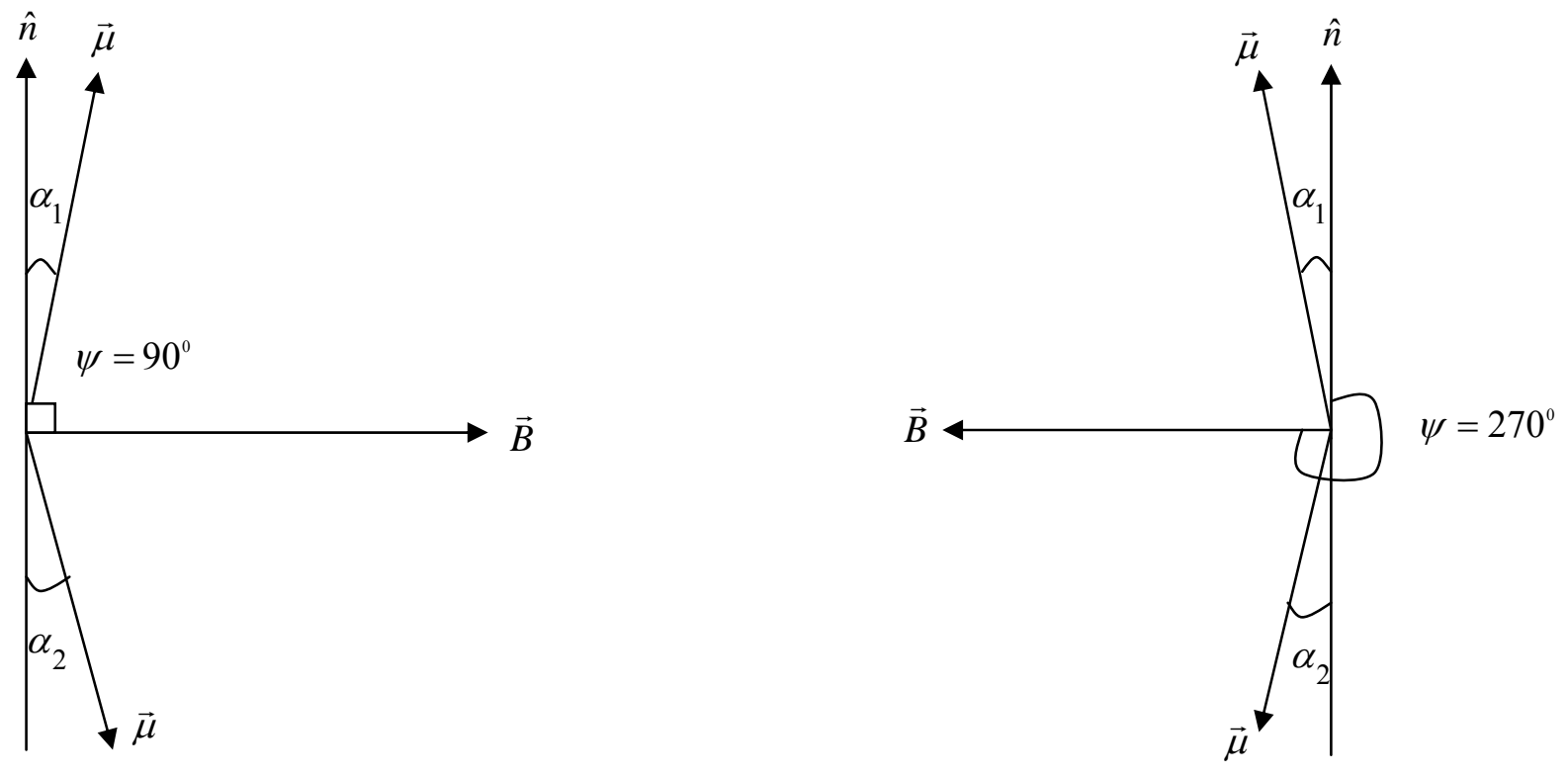

$$
\begin{aligned}
& \alpha_{1}=\frac{x}{2}, E_{1}=-K V \\
& \alpha_{2}=\pi-\frac{x}{2}, E_{2}=-K V
\end{aligned}
$$$$
\alpha_{1}=-\frac{x}{2}, E_{1}=-K V
$$$$
\alpha_{2}=\pi+\frac{x}{2}, E_{2}=-K V
$$

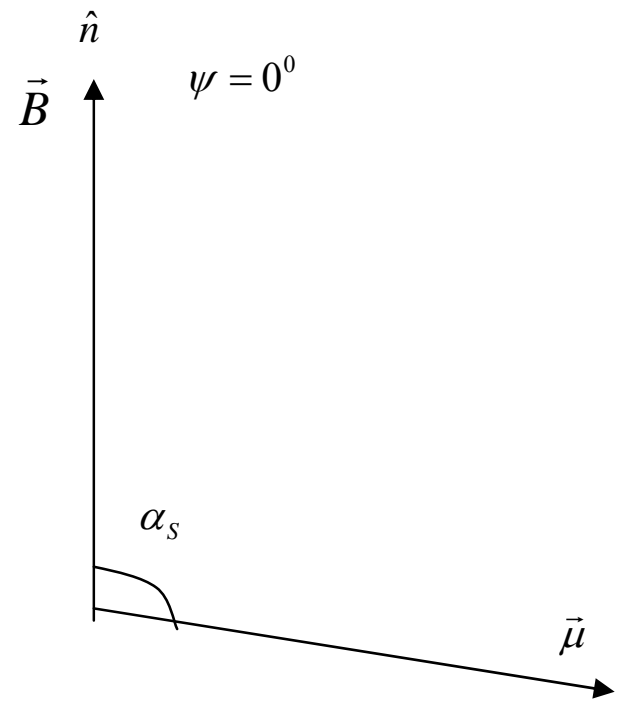

$$
\alpha_{S}=\frac{\pi}{2}+\frac{x}{2}, E_{1}=0
$$

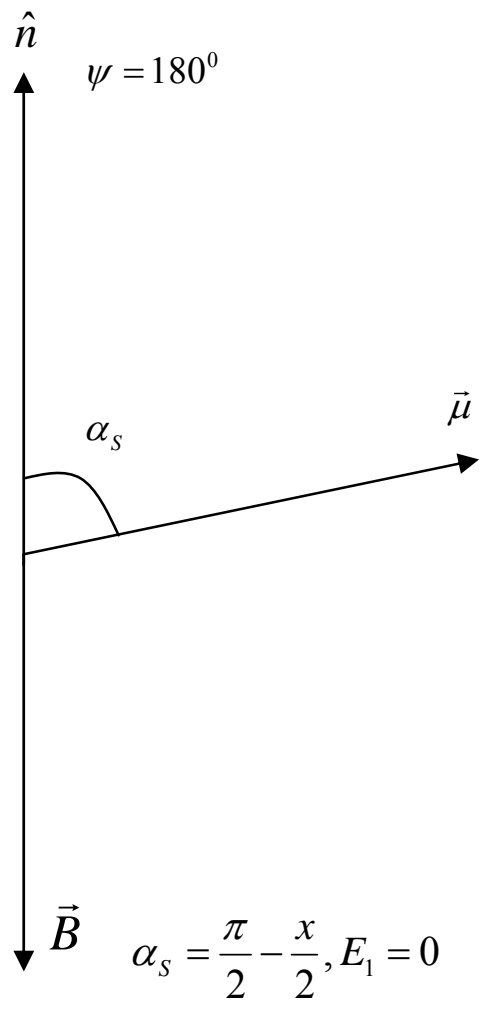


We calculate the z-component of the particle magnetization along the direction of the external magnetic field for the angle $\alpha_{1}=\frac{x}{2} \sin (\psi)$ denoted by $\mathrm{m}_{1}$ starting from:

$$
\begin{aligned}
m_{1} & =M_{S} \cos \left(\psi-\alpha_{1}\right) \\
& =M_{S}\left(\cos (\psi) \cos \left(\alpha_{1}\right)+\sin (\psi) \sin \left(\alpha_{1}\right)\right) \\
& \approx M_{S}\left(\cos (\psi)+\sin (\psi) \alpha_{1}\right) \\
m_{1} & \approx M_{S}\left(\cos (\psi)+\sin ^{2}(\psi) \frac{X}{2}\right)
\end{aligned}
$$

For the angle $\alpha_{2}=\pi-\frac{x}{2} \sin (\psi), \mathrm{m}_{2}$ can be obtained as:

$$
\begin{aligned}
m_{2} & =M_{S} \cos \left(\psi-\alpha_{2}\right) \\
& =M_{S}\left(\cos (\psi) \cos \left(\alpha_{2}\right)+\sin (\psi) \sin \left(\alpha_{2}\right)\right) \\
& =M_{S}\left(\cos (\psi) \cos \left(\pi-\frac{x}{2} \sin (\psi)\right)+\sin (\psi) \sin \left(\pi-\frac{x}{2} \sin (\psi)\right)\right) \\
& =M_{S}\left(-\cos (\psi) \cos \left(\frac{x}{2} \sin (\psi)\right)+\sin (\psi) \sin \left(\frac{x}{2} \sin (\psi)\right)\right) \\
& \approx M_{S}\left(-\cos (\psi)+\frac{x}{2} \sin ^{2}(\psi)\right) \\
m_{2} & \approx M_{S}\left(-\cos (\psi)+\frac{x}{2} \sin ^{2}(\psi)\right)
\end{aligned}
$$

Néel Arrhenius equation for particles with single attempt frequency is given by $f=f_{o} e^{-E_{a} / k_{B} T}$ with $f_{o}$ as the attempt frequency and $E_{a}$ as the activation energy barrier. We define the relaxation rate as $\tau=1 / f_{o}$ in order to obtain:

$$
\frac{1}{\tau}=\frac{1}{\tau_{o}} e^{-E_{a} / k_{B} T} \text { so } \tau=\tau_{o} e^{E_{a} / k_{B} T}
$$

In order for the moments at the saddle points to cross the energy barrier with the same attempt frequency and to align along the direction of the magnetic field we define: 


$$
\tau=\tau_{o} e^{\left(E_{S}-E_{1}\right) / k_{B} T}+\tau_{o} e^{\left(E_{S}-E_{2}\right) / k_{B} T}
$$

In the absence of a saddle point $\tau$ would simply be $\tau=\tau_{o} e^{E_{1} / k_{B} T}+\tau_{o} e^{E_{2} / k_{B} T}$ for particle moments lying in the directions corresponding to $\alpha_{1}$ and $\alpha_{2}$ and their energy barriers being $E_{1}$ and $E_{2}$. Substituting for energies obtained and taking in account the low $\mathrm{x}$-limit:

$$
\begin{gathered}
\tau=\frac{\tau_{o}}{-(-K V x \sin \psi-(-K V(1+x \cos \psi))) / k_{B} T+e^{-(-K V x \sin \psi-(-K V(1-x \cos \psi)))} / k_{B} T} \\
e^{\tau=\frac{\tau_{o}}{-K V / k_{B} T}+e^{-K V / k_{B} T}} ; \tau=\frac{\tau_{o}}{2} e^{K V / k_{B} T}
\end{gathered}
$$

In order to calculate the fraction of moments that lie in the $\mathrm{m}_{1}$ direction we define:

$$
\begin{aligned}
n_{e q} & =\frac{1}{1+e^{-\left(E_{2}-E_{1}\right) / k_{B} T} \text { so if } E_{1}=E_{2}, n_{e q}=\frac{1}{2}} \\
& =\frac{1}{1+\left(-K V(1-x \cos \psi)-(-K V(1+x \cos \psi)) / k_{B} T\right.} \\
& =\frac{1}{1+e^{-(-K V+K V x \cos \psi+K V+K V x \cos \psi) / k_{B} T}} \\
& =\frac{1+e^{-(2 K V x \cos \psi) / k_{B} T}}{n_{e q} \approx \frac{1}{1+1-\frac{2 K V x \cos \psi}{k_{B} T}} \approx \frac{2 K V x \cos \psi}{k_{B}}} \approx\left(2-\frac{2 K V x \cos \psi}{k_{B} T}\right)^{-1} \approx \frac{1}{2}\left(1+\frac{K V x \cos \psi}{k_{B} T}\right)
\end{aligned}
$$

Where we have used the expansion for $e^{x}=1+x$ in order to obtain:

$$
n_{e q} \approx \frac{1}{2}\left(1+\frac{K V x \cos \psi}{k_{B} T}\right)
$$


The fraction of moments are given by $n^{+}(t)=n_{e q}+\left(\frac{1}{2}-n_{e q}\right) P(t)$; where $P(t)=e^{-t / \tau}$ denotes that the magnetization has not switched after a time $t$, so the expression becomes $n^{+}(t)=n_{e q}+\left(\frac{1}{2}-n_{e q}\right) e^{-t / \tau}$. The magnetization is given by multiplying the fraction of moments that lie in the $\mathrm{m}_{1}$ times the moments in that direction plus $\mathrm{m}_{2}$ times the fraction of moments that lie in the other direction represented by $\mathrm{m}(\mathrm{t})$ below:

$$
\begin{aligned}
& m(t)=n^{+}(t) m_{1}+\left(1-n^{+}(t)\right) m_{2} \\
& m(t)=\left[n_{e q}+\left(\frac{1}{2}-n_{e q}\right) e^{-t / \tau}\right] M_{S}\left[\cos (\psi)+\frac{x}{2} \sin ^{2}(\psi)\right]+\left[1-\left(n_{e q}+\left(\frac{1}{2}-n_{e q}\right) e^{-t / \tau}\right)\right] M_{S}[-\cos (\psi) \\
& \left.+\frac{x}{2} \sin ^{2}(\psi)\right] \\
& =M_{. S}\left[n_{e q} \cos (\psi)+\left(\frac{1}{2}-n_{e q}\right) e^{-t / \tau} \cos (\psi)+\frac{n_{e q}}{2} \sin ^{2}(\psi)+\left(\frac{1}{2}-n_{e q}\right) e^{-t / \tau} \frac{x}{2} \sin ^{2}(\psi)-\cos (\psi)\right. \\
& +\frac{x}{2} \sin ^{2}(\psi)-\left(n_{e q}+\left(\frac{1}{2}-n_{e q}\right) e^{-t / \tau}\right)\left(-\cos (\psi)+\frac{x}{2} \sin ^{2}(\psi)\right) \\
& =M_{. S}\left[n_{e q} \cos (\psi)+\left(\frac{1}{2}-n_{e q}\right) e^{-t / \tau} \cos (\psi)+\frac{n_{e q}}{2} \sin ^{2}(\psi)+\left(\frac{1}{2}-n_{e q}\right) e^{-t / \tau} \frac{x}{2} \sin ^{2}(\psi)-\cos (\psi)\right. \\
& \left.+\frac{x}{2} \sin ^{2}(\psi)+n_{e q} \cos (\psi)+\left(\frac{1}{2}-n_{e q}\right) e^{-t / \tau} \cos (\psi)-n_{e q} \frac{x}{2} \sin ^{2}(\psi)\right)-\left(\frac{1}{2}-n_{e q}\right) e^{-t / \tau} \frac{x}{2} \sin ^{2}(\psi) \\
& =M_{S}\left[\cos (\psi)\left(n_{e q}+\left(\frac{1}{2}-n_{e q}\right) e^{-t / \tau}-1+n_{e q}+\left(\frac{1}{2}-n_{e q}\right) e^{-t / \tau}\right)+x \sin ^{2}(\psi)\left(\frac{n_{e q}}{2}+\frac{1}{2}\left(\frac{1}{2}-n_{e q}\right) e^{-t / \tau}\right.\right. \\
& \left.+\frac{1}{2}-\frac{n_{e q}}{2}-\frac{1}{2}\left(\frac{1}{2}-n_{e q}\right) e^{-t / \tau}\right] \\
& =M_{S}\left[\cos (\psi)\left(2 n_{e q}+\left(1-2 n_{e q}\right) e^{-t / \tau}-1\right)+\frac{x}{2} \sin ^{2}(\psi)\right] \\
& m(t)=M_{S}\left[\cos (\psi)\left(2 n_{e q}-1\right)\left(1-e^{-t / \tau}\right)+\frac{x}{2} \sin ^{2}(\psi)\right]
\end{aligned}
$$
reveals:

$$
m(t)=\frac{\mu_{O} M^{2}{ }_{S} H_{a}}{K}\left[\cos ^{2}(\psi) \frac{K V}{k_{B} T}\left(1-e^{-t / \tau}\right)+\frac{1}{2} \sin ^{2}(\psi)\right]
$$

Since the easy axis can be randomly oriented we need to average over $\psi$. 


$$
\frac{2 \pi \int_{0}^{\pi} \cos ^{2}(\psi) \sin (\psi) d \psi}{2 \pi \int_{0}^{\pi} \sin (\psi) d \psi}=\frac{1}{3} ; \quad \frac{2 \pi \int_{0}^{\pi} \sin ^{2}(\psi) \sin (\psi) d \psi}{2 \pi \int_{0}^{\pi} \sin (\psi) d \psi}=\frac{2}{3}
$$

This simplifies the expression for $\mathrm{m}(\mathrm{t})$ as from where we can obtain $\chi$ :

$$
\begin{aligned}
& m(t)=\left[\frac{\mu_{O} M^{2}{ }_{S} H_{a}}{K}\left[\frac{1}{3} \frac{K V}{k_{B} T}\left(1-e^{-t / \tau}\right)+\frac{1}{3}\right]\right] \\
& \chi(t)=\frac{m(t)}{H_{a}}=\frac{\mu_{O} M^{2} S}{3 K}\left[\frac{K V}{k_{B} T}\left(1-e^{-t / \tau}\right)+1\right]
\end{aligned}
$$

The AC susceptibility $\chi(\omega)=\int_{0}^{\infty} \frac{d \chi}{d t} e^{-i \omega t} d t$ here $\frac{d \chi}{d t}=\frac{\mu_{0} M_{S}^{2}}{3 K}\left[\delta(t)+\frac{K V}{k_{B} T \tau} e^{-t / \tau}\right]$.

The presence of a delta function comes from the fact that the two energy minima shift towards the direction of the applied field as soon as the field is applied. This gives rise to net magnetization which leads to a non-zero contribution to the susceptibility.

$$
\begin{aligned}
& \chi(\omega)=\frac{\mu_{0} M^{2} s^{\infty}}{3 K} \int_{0}^{\infty}\left(\delta(t)+\frac{K V}{k_{B} T \tau} e^{-t / \tau}\right) e^{-i \omega t} d t=\frac{\mu_{0} M^{2} s}{3 K}\left[1+\frac{K V}{k_{B} T \tau} \frac{e^{-\left(\frac{1}{\tau}+i \omega\right) t}-\left(\frac{1}{\tau}+i \omega\right)}{]_{0}^{\infty}}\right. \\
& =\frac{\mu_{o} M^{2} s}{3 K}\left[1+\frac{K V}{k_{B} T} \frac{0-1}{-(1+i \omega \tau)}\right]=\frac{\mu_{0} M^{2} s}{3 K}\left[1+\frac{K V}{k_{B} T} \frac{1}{(1+i \omega \tau)}\right] \\
& =\frac{\mu_{0} M^{2} s}{3 K}\left[1+\frac{K V}{k_{B} T} \frac{1}{(1+i \omega \tau)} \frac{(1-i \omega \tau)}{(1-i \omega \tau)}\right]=\frac{\mu_{0} M^{2} s}{3 K}\left[1+\frac{K V}{k_{B} T} \frac{(1-i \omega \tau)}{\left(1+(\omega \tau)^{2}\right)}\right] \\
& =\frac{\mu_{o} M^{2} s}{3 K}\left[1+\frac{K V}{k_{B} T}\left[\frac{1}{1+(\omega \tau)^{2}}-\frac{i \omega \tau}{1+(\omega \tau)^{2}}\right]\right]==\frac{\mu_{o} M^{2} s}{3 K}\left[1+\frac{K V}{k_{B} T} \frac{1}{1+(\omega \tau)^{2}}-i \frac{K V}{k_{B} T} \frac{\omega \tau}{1+(\omega \tau)^{2}}\right]
\end{aligned}
$$

from $\chi(\omega)=\chi^{\prime}-i \chi^{\prime \prime}$ we obtain the expressions for:

$$
\begin{aligned}
& \chi^{\prime}=\frac{\mu_{0} M^{2} s}{3 K}\left[1+\frac{K V}{k_{B} T} \frac{1}{1+(\omega \tau)^{2}}\right] \\
& \chi^{\prime \prime}=\frac{\mu_{0} M^{2} s}{3 K}\left[\frac{K V}{k_{B} T} \frac{\omega \tau}{1+(\omega \tau)^{2}}\right]
\end{aligned}
$$




\section{APPENDIX III}

\section{Derivation for Anisotropy Field}

In this appendix a part of the calculation for the temperature dependence of the anisotropy field is followed up on from Eq. 6.8 given below leading to Eq. 6.9 and Eq. 6.10 from chapter 6 . The equations have been followed up from the paper by [Biasi et al, 1978].

$$
\begin{gathered}
\frac{H_{A}{ }^{S P}}{H_{A}}=\frac{\left\langle P_{n}(\cos \theta)\right\rangle}{\langle\cos \theta\rangle} \text { with } H_{A}=K / I_{S} \\
H_{A}{ }^{S P}(\text { Axial })=H_{A} \frac{1-3 x^{-1} \operatorname{coth}(x)+3 x^{-2}}{\operatorname{coth}(x)-x^{-1}} \\
H_{A}{ }^{S P} \text { (Cubic) }=H_{A} \frac{1-10 x^{-1} \operatorname{coth}(x)+45 x^{-2}-105 x^{-3} \operatorname{coth}(x)+105 x^{-4}}{\operatorname{coth}(x)-x^{-1}}
\end{gathered}
$$

The energy of a sample in the presence of an external magnetic field is given by:

$$
E=-M \cdot H=-M H \cos \theta
$$

where $\theta$ is the angle between moment and the applied field. Neglecting the discreteness of the allowed energy levels and carrying out average over all positions.

$$
\langle\cos \theta\rangle=\frac{\int_{0}^{\pi} \sin \theta \cos \theta e^{\frac{M H \cos \theta}{k T}} d \theta}{\int_{0}^{\pi} \sin \theta e^{\frac{M H \cos \theta}{k T}} d \theta}
$$

This integral can be evaluated by denoting $\mathrm{x}=\cos \theta, \mathrm{dx}=-\sin \theta \mathrm{d} \theta$ and $\mathrm{a}=\mathrm{MH} / \mathrm{kT}$ to obtain:

$$
\langle x\rangle=\frac{\int_{-1}^{1} x e^{a x} d x}{\int_{-1}^{1} e^{a x} d x}=\operatorname{coth} a-\frac{1}{a}
$$


Axial symmetry $(\mathrm{n}=2)$ :

$$
\begin{gathered}
P_{2}(\cos \theta)=\frac{3}{2} \cos ^{2} \theta-\frac{1}{2} \\
\left\langle P_{2}(\cos \theta)\right\rangle=\frac{\int_{0}^{\pi} \sin \theta\left(\frac{3}{2} \cos ^{2} \theta-\frac{1}{2}\right) e^{a \cos \theta} d \theta}{\int_{0}^{\pi} \sin \theta e^{a \cos \theta} d \theta} \\
\left\langle P_{2}(x)\right\rangle=\frac{\int_{-1}^{1}\left(\frac{3}{2} x^{2}-\frac{1}{2}\right) e^{a x} d x}{\int_{-1}^{1} e^{a x} d x}=1+\frac{3}{a^{2}}-\frac{3}{a} \operatorname{coth} a
\end{gathered}
$$

Cubic symmetry $(n=4)$ :

$$
\begin{gathered}
P_{4}(\cos \theta)=\frac{1}{8}\left(35 \cos ^{4} \theta-30 \cos ^{2} \theta+3\right) \\
\left\langle P_{4}(\cos \theta)\right\rangle=\frac{\int_{0}^{\pi} \sin \theta\left(\frac{1}{8}\left(35 \cos ^{4} \theta-30 \cos ^{2} \theta+3\right)\right) e^{a \cos \theta} d \theta}{\int_{0}^{\pi} \sin \theta e^{a \cos \theta} d \theta} \\
\left\langle P_{4}(x)\right\rangle=\frac{\int_{-1}^{1}\left(\frac{1}{8}\left(35 x^{4}-30 x^{2} \theta+3\right)\right) e^{a x} d x}{\int_{-1}^{1} e^{a x} d x}=1+\frac{45}{a^{2}}-\frac{105}{a^{4}}-\frac{10}{a} \operatorname{coth} a-\frac{105}{a^{3}} \operatorname{coth} a
\end{gathered}
$$

Upon substituting the Eqs. (III.5), (III.8) and (III.11) in (III.1) one obtains the case for axial and cubic symmetry. 